\title{
Experiments on the Self-Organized Critical State of ${ }^{4} \mathrm{He}$
}

\author{
Thesis by \\ Andrew Rosenberg Chatto \\ In Partial Fulfillment of the Requirements \\ for the Degree of \\ Doctor of Philosophy
}

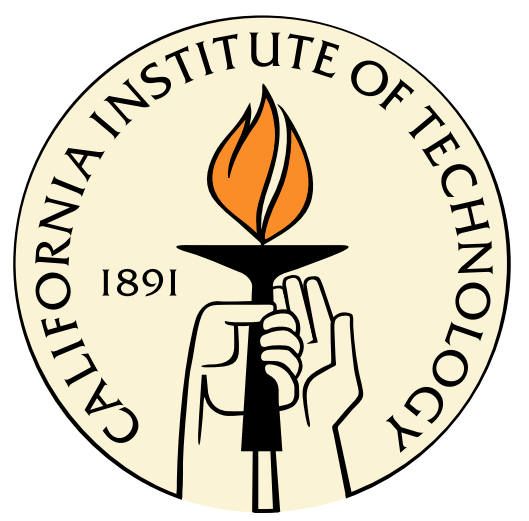

California Institute of Technology

Pasadena, California

2006

(Defended April 12, 2006) 
(C) 2006

Andrew Rosenberg Chatto

All Rights Reserved 


\section{Acknowledgements}

There are a number of people I would like to thank for their contributions to this thesis. My advisor David Goodstein was insightful, encouraging, and patient throughout the many challenges of performing a low-temperature experiment. Our conversations were always helpful because David has an extraordinary ability to make complicated concepts seem simple and intuitive. I had two mentors in the lab, Peter Day and Richard Lee, who taught me everything I know about performing experiments in low-temperature physics. My first lab experience was working with Peter. He provided me with the cryostat I used throughout my graduate career, answered hundreds of questions, and patiently showed me the techniques I needed. Richard returned to Caltech as my thesis experiment was taking shape. He was also an excellent resource for the tricks and techniques of low-temperature physics. (Plus, he made B.O.B.2, the electrical filtering system on this cryostat). However, what helped me most was that he patiently asked hundreds of questions, which forced me to think through all the details of my experiment. It is with his help that I avoided many pitfalls.

There are a number of other physicists who made significant contributions to this work. Particularly helpful were the members of the DYNAMX team from the University of New Mexico (Rob Duncan, Dimitri Sergatskov, Alex Babkin, and Steve Boyd). They provided material help with the cryo-valve system and the construction of the experimental cell. In addition, they provided a lot of expertise on performing experiments on the SOC state of ${ }^{4} \mathrm{He}$. In particular, I would like to thank Rob Duncan, who served as a secondary thesis advisor during a critical time in the experiment as David Goodstein recovered from an injury. Also helpful were discussions with two theorists, Peter Weichman and Rudolf Haussmann, who helped give meaning to my results.

I would like to thank my parents for many many years of encouragement and support, and not asking me too often when I was going to get my degree.

I would also like to thank my wife Avital, who provided an enormous amount of support and, through an intricate dance of prodding and patience, helped guide me through this endeavor. Lastly, I thank my son Jacob, whose arrival helped destroy the illusion that maybe, just maybe, I could be a graduate student forever. 


\section{Abstract}

When a heat flux is applied downwards through a sample of ${ }^{4} \mathrm{He}$ near the superfluid transition temperature $T_{\lambda}$, the gradient in the temperature self-organizes to the gradient in $T_{\lambda}$ caused by gravity. This creates the Self-Organized Critical (SOC) state. Previous experiments have observed the state, measured the self-organization temperature $T_{\mathrm{SOC}}$ vs. heat flux, and investigated a remarkable wave that only travels upwards against the flow of the heat flux $[1,2,3]$.

We report the first results of the heat capacity of the SOC state, $C_{\nabla T}$, for heat fluxes $60 \mathrm{nW} / \mathrm{cm}^{2}<$ $Q<13 \mu \mathrm{W} / \mathrm{cm}^{2}$ and corresponding temperatures $9 \mathrm{nK}>T_{\mathrm{SOC}}-T_{\lambda}>-1.1 \mu \mathrm{K}$. We find that $C_{\nabla T}$ tracks the static (i.e., zero heat flux) unrounded (i.e., in zero gravity) heat capacity $C_{0}$ with two exceptions. The first is that within $250 \mathrm{nK}$ of $T_{\lambda}, C_{\nabla T}$ is depressed relative to $C_{0}$ and the maximum in $C_{\nabla T}$ is shifted to $50 \mathrm{nK}$ below $T_{\lambda}$. The second difference is that at high heat flux, $C_{\nabla T}$ is again depressed relative to $C_{0}$ with the departure starting at about $650 \mathrm{nK}$ below $T_{\lambda}$.

We present the most extensive measurements of the speed and attenuation of the SOC wave to date. We report wave speed measurements taken over our full experimental range $30 \mathrm{nW} / \mathrm{cm}^{2}<$ $Q<13 \mu \mathrm{W} / \mathrm{cm}^{2}$ and attenuation results over the limited range that produced enough attenuation to measure. We also report the first accurate calculation of the speed of the SOC wave. 


\section{Contents}

Acknowledgements $\quad$ iii

Abstract $\quad$ iv

Table of Contents $\quad$ v

List of Tables $\quad$ vii

List of Figures $\quad$ viii

1 Introduction 1

1.1 The SOC State . . . . . . . . . . . . . . . . . . . . . 1

1.2 The SOC Heat Capacity . . . . . . . . . . . . . . . . . . . . 3

1.3 The SOC State Wave . . . . . . . . . . . . . . . . . . . . 5

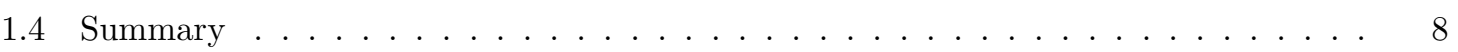

2 Apparatus 9

2.1 Cryogenic System . . . . . . . . . . . . . . . . . . . . . . . . . 9

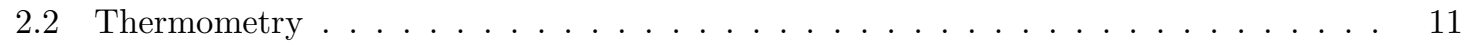

2.3 Auxiliary Stages . . . . . . . . . . . . . . . . . . . . . . . . 11

2.4 Cell Stage . . . . . . . . . . . . . . . . . . . . . . . . . . . . 13

2.4.1 Mounting Platform . . . . . . . . . . . . . . . . . . . . 13

2.4 .2 Cell Construction . . . . . . . . . . . . . . . . . . . . . . 14

2.4.3 Fill Line and Bubble Chamber . . . . . . . . . . . . . . . . . . . 15

2.4 .4 Cryo-Valve and ${ }^{3} \mathrm{He}$ Actuation System . . . . . . . . . . . . . . . . 15

2.4 .5 HRT Mounting . . . . . . . . . . . . . . . . . . . . 15

2.4 .6 GRT Mounting . . . . . . . . . . . . . . . . . . 16

2.4 .7 Heaters . . . . . . . . . . . . . . . . . . . . . . . 16

2.5 Data Acquisition and Experiment Control . . . . . . . . . . . . . . . . . . 16

$2.5 .1 \quad$ Cryo-Probe Inputs and Outputs _ . . . . . . . . . . . . . . . . 16 
2.5.2 Data Acquisition and Control . . . . . . . . . . . . . . . . 17

2.5.3 Computer Inputs and Outputs _ . . . . . . . . . . . . . . . . 18

2.5.4 Computer Software . . . . . . . . . . . . . . . . . . . 18

2.5.4.1 General Overview . . . . . . . . . . . . . . . . . . 18

2.5.4.2 Flux Counting . . . . . . . . . . . . . . . . . 19

3 Calibration 21

3.1 Thermal Network . . . . . . . . . . . . . . . . . . . . . . . . . 21

3.2 Heater Calibrations . . . . . . . . . . . . . . . . . . . . . . 21

3.3 Endplate Heat Capacity . . . . . . . . . . . . . . . . . . . . . . . . . 22

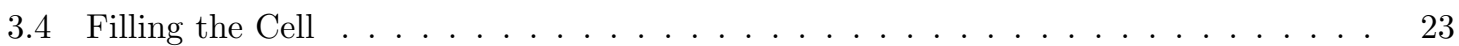

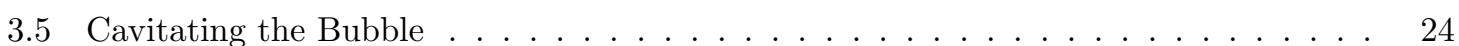

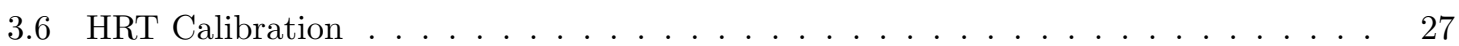

3.7 HRT Noise and Drift . . . . . . . . . . . . . . . . . . . . . . . . 29

3.8 Kapitza Resistance . . . . . . . . . . . . . . . . . . . . . . . . . . . . . . . . . 31

3.9 Heater Response Times . . . . . . . . . . . . . . . . . . . . . . . . . . . . . . . . 31

3.9 .1 The Problem . . . . . . . . . . . . . . . . . . . . . 31

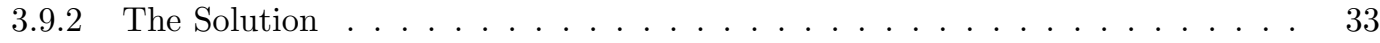

3.10 Heat Leaks . . . . . . . . . . . . . . . . . . . . . . . 33

3.10 .1 The Problem . . . . . . . . . . . . . . . . . . . . . . 35

3.10 .2 The Solution . . . . . . . . . . . . . . . . . . . . . . 36

3.11 Static Heat Capacity . . . . . . . . . . . . . . . . . . . . . . . . . . . 37

3.12 Emptying the Cell . . . . . . . . . . . . . . . . . . . . . . . . . . 39

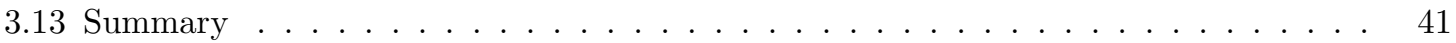

4 SOC Heat Capacity 43

4.1 Procedure . . . . . . . . . . . . . . . . . . . . . . . . . . . 43

4.1 .1 Procedure Overview . . . . . . . . . . . . . . . . . . . . 43

4.1 .2 Maintaining the Balance . . . . . . . . . . . . . . . . . . . . . 45

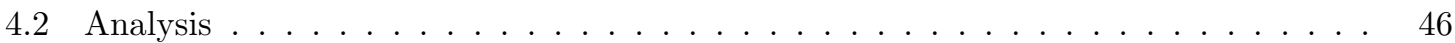

4.2 .1 SOC Temperature Change . . . . . . . . . . . . . . . . . . . 46

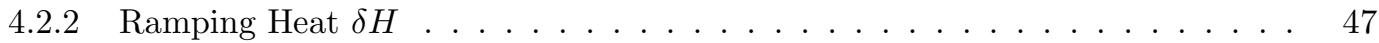

4.2 .3 Time Interval . . . . . . . . . . . . . . . . . . . . . . . . . 47

4.2 .4 The Uncorrected Heat Capacity _ . . . . . . . . . . . . . . . . . . . . 48

4.2 .5 Heater Response Time Correction _ . . . . . . . . . . . . . . . . . . 50

4.2 .6 Foil Heat Leak . . . . . . . . . . . . . . . . . . . . . . . . . 50

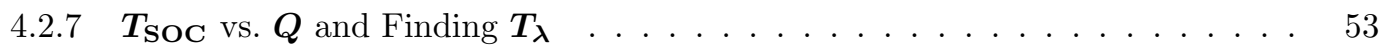


4.2.8 Effect of Different Experimental Parameters . . . . . . . . . . . . . . 55

4.2 .9 Maintaining the Balance, Revisited . . . . . . . . . . . . . . . 55

4.3 Results and Conclusion $\ldots \ldots \ldots \ldots \ldots \ldots \ldots$

4.3 .1 Results . . . . . . . . . . . . . . . . . . . . . 58

4.3 .2 Comparison with Theory _ . . . . . . . . . . . . . . . . . . 60

4.3 .3 Conclusion . . . . . . . . . . . . . . . . . . . . . . 61

$5 \quad$ The SOC Wave $\quad 63$

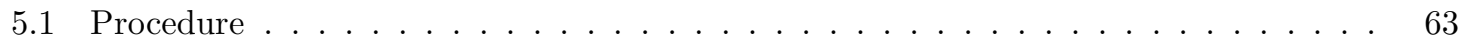

5.2 Analysis . . . . . . . . . . . . . . . . . . . . 64

5.2 .1 Phase and Amplitude . . . . . . . . . . . . . . . . . . . 64

$5.2 .2 \quad$ SOC Layer Height $\ldots \ldots \ldots \ldots$. . . . . . . . . . . . . . 67

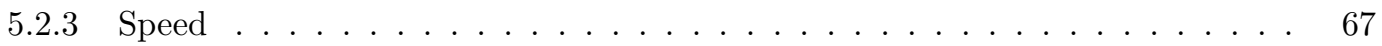

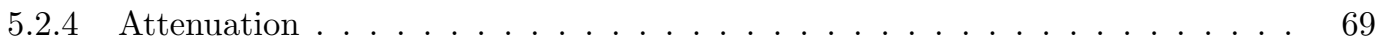

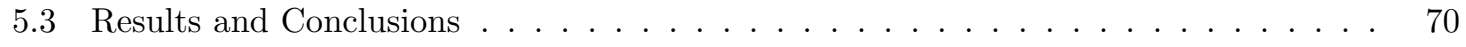

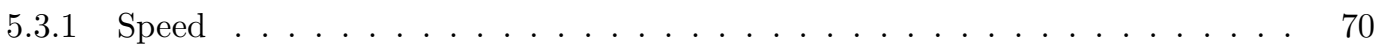

5.3 .2 Attenuation . . . . . . . . . . . . . . . . . . 71

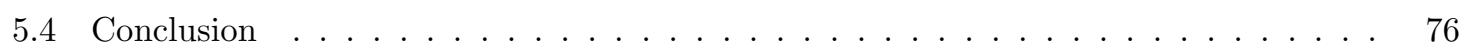

$\begin{array}{lll}6 & \text { Summary } & 79\end{array}$

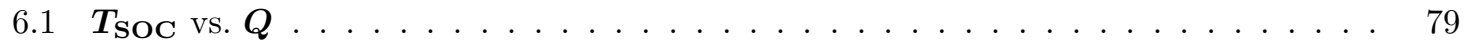

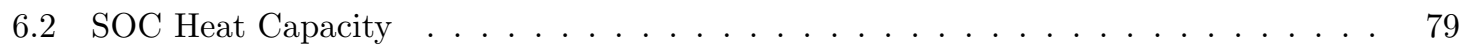

6.3 SOC Wave . . . . . . . . . . . . . . . . . . . . . . . . 82

$\begin{array}{lr}\text { Bibliography } & 85\end{array}$

$\begin{array}{lr}\text { A Cell Drawings } & 87\end{array}$ 


\section{List of Tables}

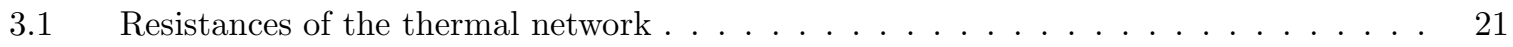

$3.2 \quad$ Heater calibrations . . . . . . . . . . . . . . . . . . . . . . 22

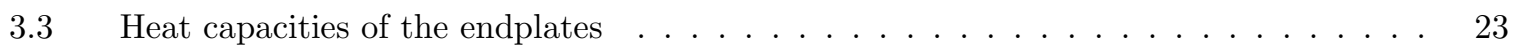

$3.4 \quad$ HRT calibrations . . . . . . . . . . . . . . . . . . . . . . 27

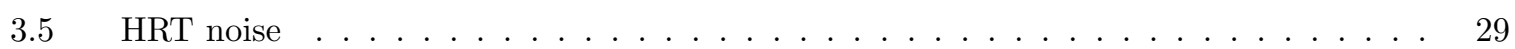

$3.6 \quad$ Kapitza resistances . . . . . . . . . . . . . . . . . . . . . . . . . 31

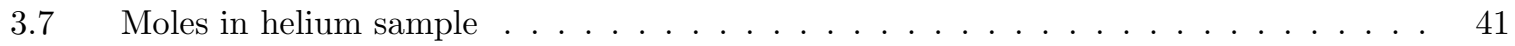

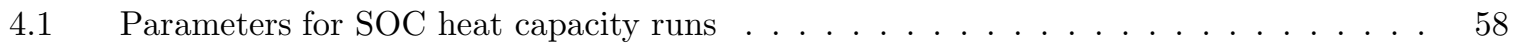

$5.1 \quad$ Parameters for a fit of $T_{\mathrm{SOC}}$ vs. $Q \ldots \ldots \ldots \ldots \ldots \ldots \ldots \ldots \ldots$ 


\section{List of Figures}

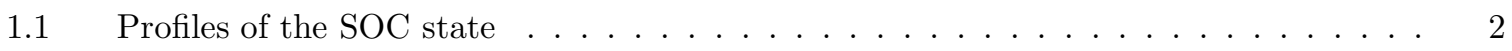

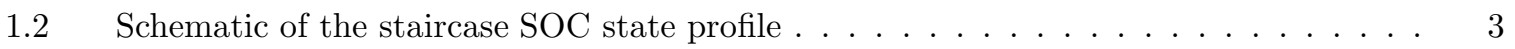

1.3 Haussmann's prediction for $C_{\nabla T}$ compared to the static heat capacity . . . . . . . . 4

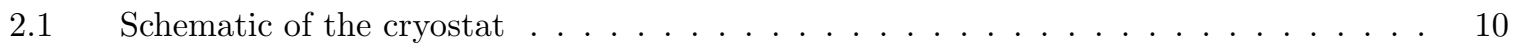

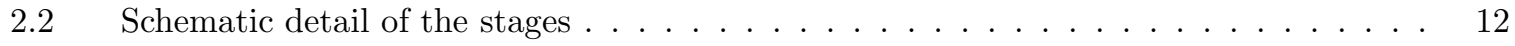

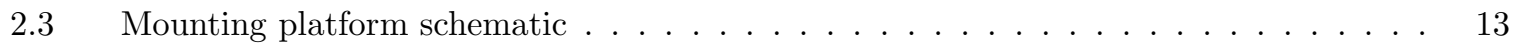

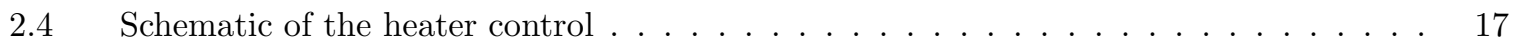

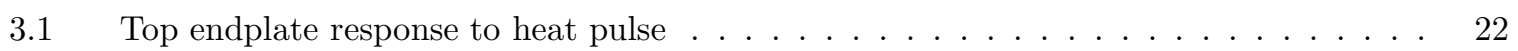

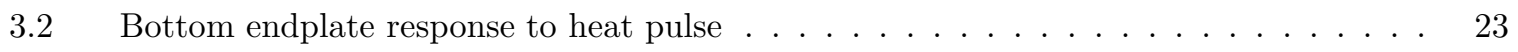

$3.3 \quad$ Cell temperature profiles when the bubble is inside the cell $\ldots \ldots \ldots \ldots$

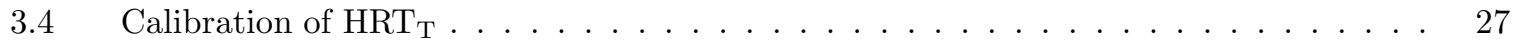

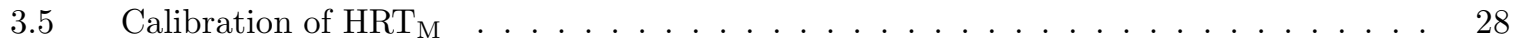

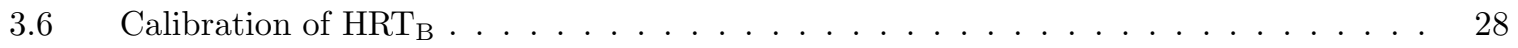

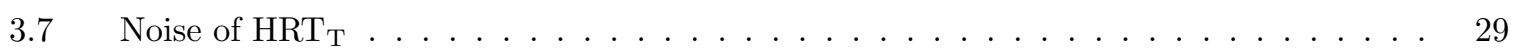

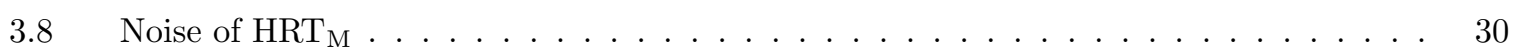

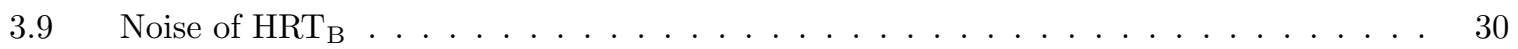

3.10 Heater response time . . . . . . . . . . . . . . . . . . . . . . 32

3.11 Measurement of cell heater response time . . . . . . . . . . . . . . . . 34

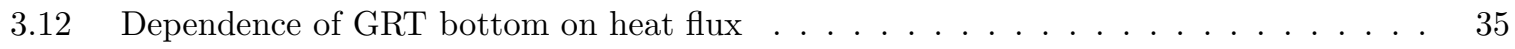

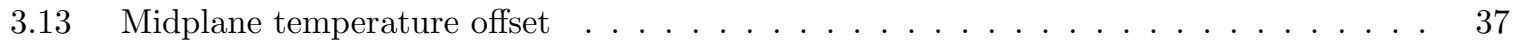

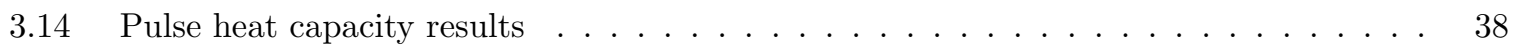

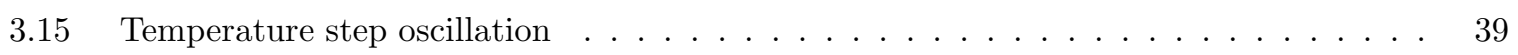

3.16 Heat capacity results with a $3.98 \mu \mathrm{J}$ pulse size $\ldots \ldots \ldots \ldots$. . . . . . . . 40

3.17 Volume calibration schematic . . . . . . . . . . . . . . . . . . . . . 40

4.1 Schematic of the temperature profiles during the SOC state heat capacity measurement 44

4.2 SOC heat capacity time series data . . . . . . . . . . . . . . . 44

$4.3 \quad$ Examples of matching the $\mathrm{HRT}_{\mathrm{T}}$ derivative curves $\ldots \ldots \ldots \ldots \ldots$ 
4.4 SOC heat capacity results for different foil heats $\ldots \ldots \ldots \ldots \ldots$. . . . . . . 51

4.5 SOC heat capacity results to determine the foil heat factor $\gamma \ldots \ldots \ldots \ldots$

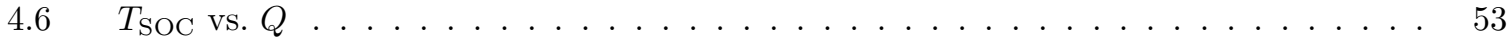

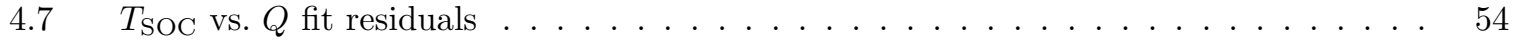

4.8 SOC heat capacity with different temperature steps $\Delta T_{\mathrm{SOC}} \ldots \ldots \ldots \ldots \ldots$

4.9 SOC heat capacity with different ramping heats $\delta H \ldots \ldots \ldots \ldots \ldots$

4.10 The effect of imbalance on the SOC heat capacity $\ldots \ldots \ldots \ldots$. . . . . . . . 57

4.11 SOC heat capacity . . . . . . . . . . . . . . . . . . . . . . . . . . . . 59

$4.12 \quad \log -\log$ plot of SOC heat capacity . . . . . . . . . . . . . . . . . . . . . 60

4.13 SOC heat capacity results compared to Haussmann's prediction . . . . . . . . . . . 61

4.14 Log-log plot of SOC heat capacity results compared to Haussmann's prediction . . . . 62

5.1 Comparison of $\mathrm{HRT}_{\mathrm{T}}$ to a reference sine wave $\ldots \ldots \ldots \ldots \ldots$

$5.2 \quad$ Amplitude vs. reference sine wave phase shift $\ldots \ldots \ldots \ldots \ldots$

$5.3 \quad \mathrm{HRT}_{\mathrm{T}}$ phase delay and $\mathrm{HRT}_{\mathrm{M}}$ phase delay vs. $\mathrm{HRT}_{\mathrm{T}} \ldots \ldots \ldots \ldots$

5.4 Second sound correction of $\mathrm{HRT}_{\mathrm{T}}$ phase delay $\ldots \ldots \ldots$. . . . . . . . . 68

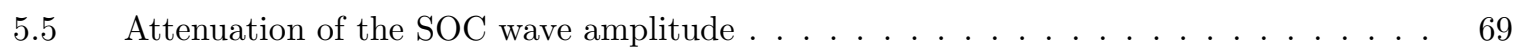

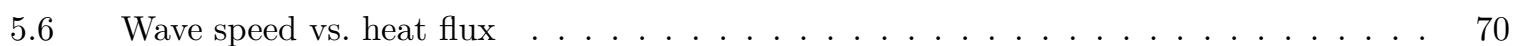

$5.7 \quad T_{\mathrm{SOC}}$ vs. $Q$ fit residuals using a fourth-degree polynomial $\ldots \ldots \ldots \ldots \ldots$

5.8 Wave speed vs. heat flux results compared to theory . . . . . . . . . . . . . . . 72

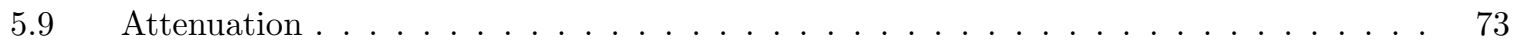

5.10 Extracting thermal conductivity from $T_{\mathrm{SOC}}$ vs. $Q$ fit residuals $\ldots \ldots \ldots \ldots$

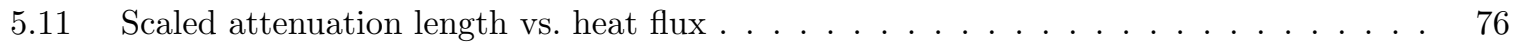

5.12 Scaled attenuation length vs. the attenuation length $\ldots \ldots \ldots \ldots \ldots$

5.13 Incorrectly scaled attenuation length vs. heat flux $\ldots \ldots \ldots \ldots$

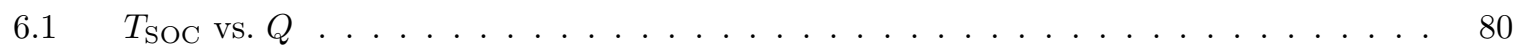

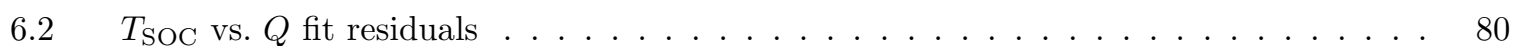

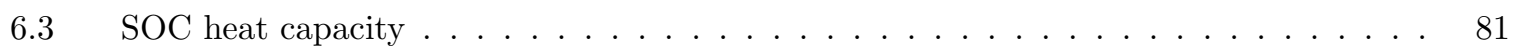

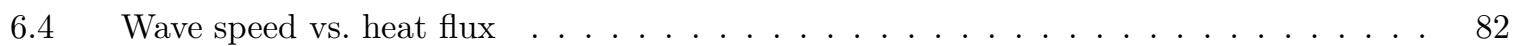

6.5 Scaled attenuation length vs. attenuation length $\ldots \ldots \ldots \ldots$

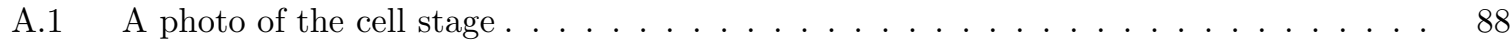

A.2 A CAD rendering of the cell stage $\ldots \ldots \ldots \ldots \ldots \ldots \ldots$

A.3 The bottom endplate . . . . . . . . . . . . . . . . . . . . 90

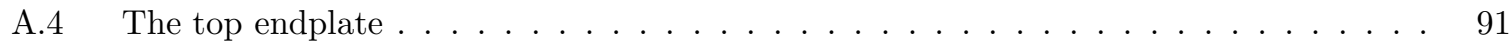

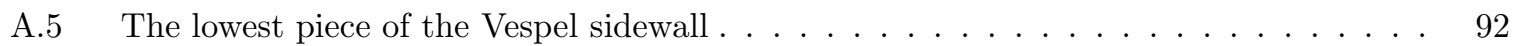




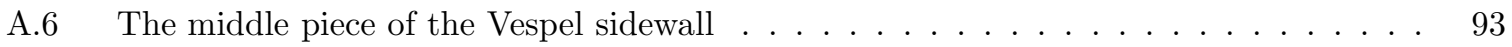

A.7 The top piece of the Vespel sidewall _ . . . . . . . . . . . . . . . . . . . . . . 94

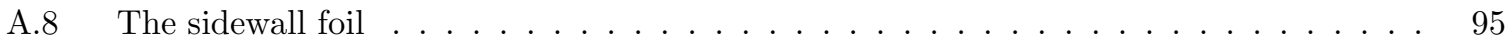

A.9 The cap for attaching the fill line to the sidewall $\ldots \ldots \ldots \ldots$

A.10 The titanium mounting plate . . . . . . . . . . . . . . . 97

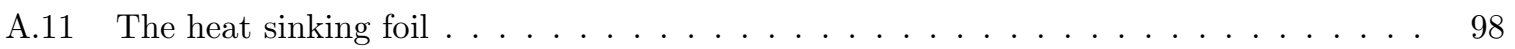

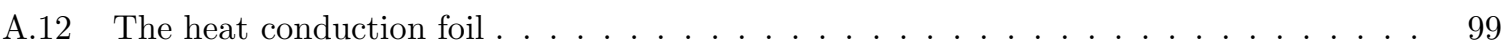

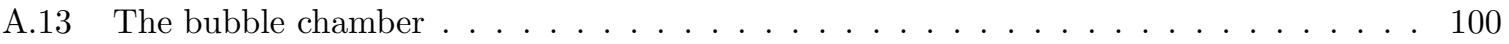

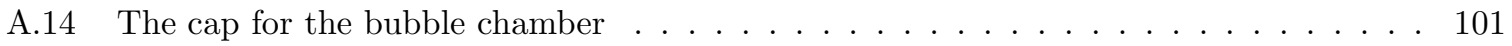

A.15 Vespel thermal standoff for the bubble chamber and cryo-valve . . . . . . . . . . . . 102

A.16 Vespel mount for the bubble chamber and cryo-valve . . . . . . . . . . . . . . . 103

A.17 Vespel mount and thermal standoff for an HRT for the bubble chamber . . . . . . . . 104 



\section{Chapter 1}

\section{Introduction}

The Self-Organized Critical (SOC) state in ${ }^{4} \mathrm{He}$ is a peculiar phenomenon arising when a heat flux is applied downwards through a sample of helium near the superfluid phase transition. Below the superfluid transition, helium has virtually no gradient under an applied heat flux as long as the heat flux does not exceed some critical value. Above the transition, the helium conducts heat as a typical fluid, although near the transition the thermal conductivity is significantly enhanced. Under certain conditions, when a heat flux is applied downwards, the helium self-organizes to a gradient of $1.273 \mu \mathrm{K} / \mathrm{cm}$ independent of the heat flux. This state is phenomenally robust; it has been seen over more than 2 orders of magnitude in heat flux (from $40 \mathrm{nW} / \mathrm{cm}^{2}$ to $6.5 \mu \mathrm{W} / \mathrm{cm}^{2}$ ) [1].

\subsection{The SOC State}

The superfluid transition temperature, $T_{\lambda}$, is depressed from its value at SVP by an imposed pressure. Therefore, a sample of helium on the surface of the Earth always has a gradient in the transition temperature of $d T_{\lambda} / d z=1.273 \mu \mathrm{K} / \mathrm{cm}$. Above $T_{\lambda}$ but near the transition, the thermal conductivity is determined by the proximity to $T_{\lambda}$ [4]. Therefore, if we define the reduced temperature $t(z)=$ $\frac{T-T_{\lambda}(z)}{T_{\lambda}}$, regions with the same reduced temperature will have the same conductivity.

Suppose we have a heat flux flowing downwards through a sample of helium. While in the superfluid, there will be no thermal gradient as in profile $\mathbf{A}$ of fig. 1.1. If we allow the entire sample to slowly warm, eventually the temperature at the bottom of the sample will pass $T_{\lambda}(z=0)$ and will begin to form a gradient. ${ }^{1}$ As the sample warms, the gradient will continue to get larger until it matches the gradient in $T_{\lambda}$ as shown by profile $\mathbf{B}$. The region of the helium where the gradient matches the gradient in $T_{\lambda}$ is in the SOC state. As the sample continues to warm, more helium will transition into the SOC state and profile $\mathbf{C}$ will be reached. When profile $\mathbf{D}$ is reached, the entire sample is in the SOC state and has the same reduced temperature $t$. As the sample warms further, a region of high gradient normal fluid will be created at the top of the sample as in profile E. The

\footnotetext{
${ }^{1}$ Actually, the gradient starts to form when the temperature passes $T_{\mathrm{C}}(Q, z) \simeq T_{\lambda}(z)-T_{\lambda}\left(Q / 784 \mathrm{~W} / \mathrm{cm}^{2}\right)^{0.813}$.
} 


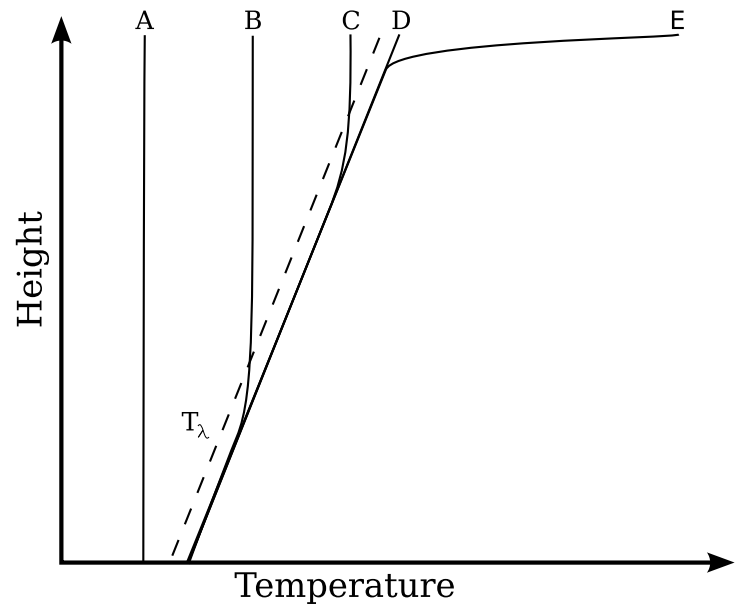

Figure 1.1. Profiles of the SOC state.

process continues as less and less helium remains in the SOC state and the high gradient normal fluid occupies more and more of the sample. Eventually, if we allow the sample to continue to warm, the arrangement will become unstable as the very warm helium at the top of the sample becomes more dense than the helium in the SOC state and convection disturbs our quasi-equilibrium.

The SOC state for normal He-I as described above was predicted by Onuki in 1987 [5]. Experiments to observe the state were conducted at the University of New Mexico by Moeur et al. and published in 1997 [1]. They saw the expected self-organization at low heat fluxes, but continued to see the SOC state for higher heat fluxes, with a self-organization temperature below $T_{\lambda}$. They presented a phenomenological model that treated the SOC state helium both above and below $T_{\lambda}$ as a normal fluid with a thermal conductivity that diverged not at $T_{\lambda}$ but at a temperature $T_{\mathrm{C}}(Q)<T_{\lambda}$. They gave the thermal conductivity $\kappa$ as

$$
\kappa(T, Q)=\kappa_{0}\left(\frac{T-T_{\mathrm{C}}(Q)}{T_{\lambda}}\right)^{-x}
$$

with $T_{\mathrm{C}}(Q)=T_{\lambda}-T_{\lambda}\left(Q / Q_{0}\right)^{0.813}$. In the SOC state, the temperature gradient equals the gradient in $T_{\lambda}$, giving $\kappa=\nabla T_{\lambda} / Q$. They used this to extract the following values for the parameters: $\kappa_{0}=294 \mathrm{nW} / \mathrm{cm} \mathrm{K}, x=0.664$, and $Q_{0}=638 \mathrm{~W} / \mathrm{cm}^{2}$. One can make an extrapolative leap and propose that this $T_{\mathrm{C}}(Q)$ is actually a depressed $T_{\lambda}(Q)$ and a true critical point. As we go to larger and larger heat flux, we need a higher thermal conductivity so the SOC temperature moves closer and closer to this critical point. We already have evidence that the conductivity $\kappa$ is diverging here. ${ }^{2}$

\footnotetext{
${ }^{2}$ In this instance, we have actually defined $T_{\mathrm{C}}(Q)$ as the temperature where $\kappa$ diverges. A previous experiment in a "heat-from-below" configuration found that $\kappa$ diverges at a temperature $T_{\mathrm{C}}(Q)<T_{\lambda}$ but that the transition was rounded [6]. They hypothesized that the rounding was due to a maximum possible size of correlated fluctuations caused by the gravity induced gradient in $T_{\lambda}$. It is unclear whether gravity would give rise to similar rounding in our experiment because the temperature gradient matches the gradient in $T_{\lambda}$
} 


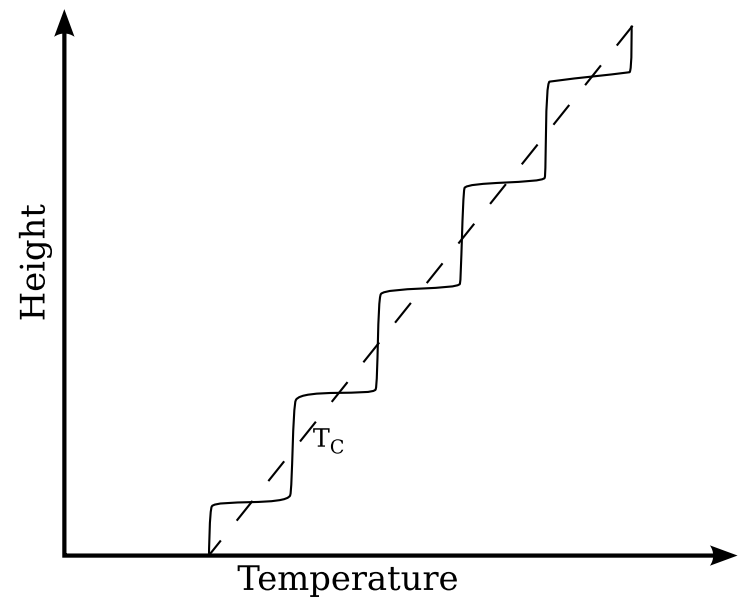

Figure 1.2. Schematic of the staircase SOC state profile in the Weichman and Miller model [7].

If we could examine other quantities as we approached $T_{\mathrm{C}}(Q)$, such as the heat capacity, we could test the hypothesis that $T_{\mathrm{C}}(Q)$ is a critical point.

Following these interesting experimental results, Weissman and Miller examined the SOC state below $T_{\lambda}$ through a one-dimensional mean-field model [7]. They calculated that it was possible to have an superfluid SOC state below $T_{\lambda}$ through a regular series of slips of the phase of the order parameter. These phase slips lead to a staircase-like temperature profile that, when viewed on a macroscopic scale, appears as a smooth temperature gradient. This is represented schematically in fig. 1.2. The main difference of this model contrasted with the proposal of a depressed $T_{\lambda}(Q)$ is that the helium is essentially a superfluid. Viewed as a whole, the helium appears as normal fluid with a uniform gradient. However, viewed on small length scales, the majority of the helium is a superfluid with no gradient.

\subsection{The SOC Heat Capacity}

Near the superfluid phase transition temperature $T_{\lambda}$, the static heat capacity (i.e., the heat capacity without a heat flux, SOC inducing or not) is dominated by the energy contribution of the random fluctuations between the two phases of helium, He-II (superfluid) and He-I (normal fluid). ${ }^{3}$ Since there is no latent heat for the phase transition, the free energy difference between the two phases goes to zero as one approaches $T_{\lambda}$ and the fluctuations between them get larger and more numerous. These fluctuations give rise to a singularity in the heat capacity of $C \sim t^{-\alpha}$ where $\alpha \simeq-0.13$.

Rudolf Haussmann used dynamic renormalization group theory to calculate the heat capacity of the SOC state, $C_{\nabla T}$ [9]. A plot of his results close to $T_{\lambda}$ are given in fig. 1.3. More than $100 \mathrm{nK}$

\footnotetext{
${ }^{3}$ For more details on the critical point phenomena near $T_{\lambda}$, see States of Matter [8] or other similar textbooks.
} 


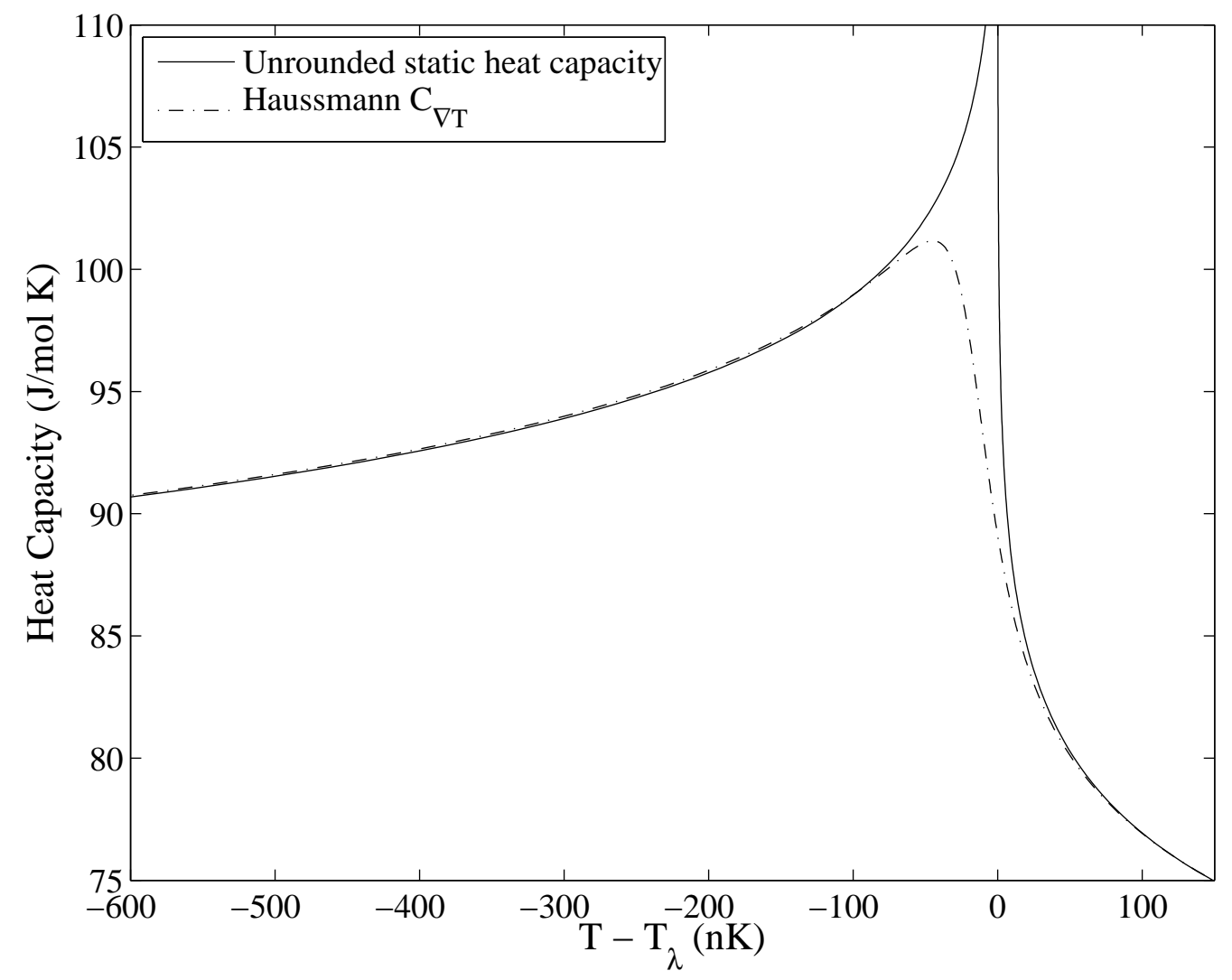

Figure 1.3. Haussmann's prediction for $C_{\nabla T}$ compared to the zero-gravity static heat capacity.

away from $T_{\lambda}$, the heat capacity is the same as in the zero-gravity static case. Very close to $T_{\lambda}$, however, the heat capacity is significantly depressed and the maximum is shifted to $50 \mathrm{nK}$ below $T_{\lambda}$.

Another prediction for $C_{\nabla T}$ comes from the suggestion that $T_{\mathrm{C}}(Q)$ might be the depressed critical point $T_{\lambda}(Q)$ with a thermal conductivity of the form given in eq. (1.1). Under this assumption, $T_{\mathrm{SOC}}(Q)$ for low $Q$ is far from the critical point. As $Q$ is increased, however, $T_{\mathrm{SOC}}(Q)$ gets closer and closer to $T_{\mathrm{C}}(Q)$. Therefore, as we approach the critical point $T_{\mathrm{C}}(Q)$ from above, we would expect the heat capacity to diverge as in the static case.

It is also possible that dynamic effects of the two-fluid model could play a role in the heat capacity. In traditional helium under a heat flux, there is predicted a contribution to the heat capacity from the kinetic energy of the flow of the superfluid [10], which has been observed experimentally in a "heat-from-below" configuration [11]. However, it is unclear whether this mechanism would apply to $C_{\nabla T}$, since it is not known how to apply the two-fluid model to the SOC state. 


\subsection{The SOC State Wave}

In investigating the SOC state for temperatures above $T_{\lambda}$, Weichman and Miller also predicted the existence of a thermal wave that would travel only upstream against the flow of the heat flux [7]. However, this wave has been seen experimentally by Sergatskov et al. both above and below $T_{\lambda}[2]$. To understand the nature of this wave, we will treat the helium as a normal fluid with a conductivity $\kappa$ that is a function of both the temperature and the heat flux. This appears to be in contradiction to the work of Weichman and Miller who treated the high heat flux SOC state as a superfluid with a regular occurrence of phase slips. Nevertheless, we will press on and defer judgment as to the validity of this approach until its predictions are compared with experimental measurements. This calculation roughly follows the calculation that Sergatskov et al. provided with their experimental paper [2].

We start with the regular heat transfer equations,

$$
C \frac{\partial T}{\partial t}=-\vec{\nabla} \cdot \vec{Q}
$$

and

$$
\vec{Q}=-\kappa \vec{\nabla} T
$$

Since the wave travels upwards and the nominal heat flux is downwards, we restrict ourselves to the

$z$ axis. We define the heat flux to be positive by setting $\vec{Q}=-Q \hat{z}$ and define $Q_{\mathrm{SOC}}$ as the nominal heat flux of the SOC state. We combine eqs. (1.2) and (1.3) to get

$$
C \frac{\partial T}{\partial t}=\frac{\partial}{\partial z}\left(\kappa \frac{\partial T}{\partial z}\right)
$$

Next, we transform to the reduced temperature $\epsilon=\left(T-T_{\lambda}\right) / T_{\lambda}$. First, we compute the derivative

$$
\frac{\partial T}{\partial z}=T_{\lambda} \frac{\partial \epsilon}{\partial z}+\alpha
$$

where $\alpha=\frac{d T_{\lambda}}{d z}=1.273 \mu \mathrm{K} / \mathrm{cm}$. Then we convert eq. (1.4) to get

$$
C T_{\lambda} \frac{\partial \epsilon}{\partial t}=\frac{\partial}{\partial z}\left(\kappa\left(T_{\lambda} \frac{\partial \epsilon}{\partial z}+\alpha\right)\right)
$$

At this point, we will avoid making the assumption that $\kappa$ is of the form given in eq. (1.1). We will, however, assume that the thermal conductivity is a function of only $\epsilon$ and $\epsilon^{\prime}=\frac{\partial \epsilon}{\partial z}$. This leads to

$$
C T_{\lambda} \frac{\partial \epsilon}{\partial t}=\left(\frac{\partial \kappa}{\partial \epsilon} \frac{\partial \epsilon}{\partial z}+\frac{\partial \kappa}{\partial \epsilon^{\prime}} \frac{\partial \epsilon^{\prime}}{\partial z}\right)\left(T_{\lambda} \frac{\partial \epsilon}{\partial z}+\alpha\right)+\kappa T_{\lambda} \frac{\partial^{2} \epsilon}{\partial z^{2}} .
$$

Stipulating that the the wave is small, we drop any terms where derivatives with respect to $\epsilon$ and $z$ 
are squared to get

$$
C T_{\lambda} \frac{\partial \epsilon}{\partial t}=\alpha \frac{\partial \kappa}{\partial \epsilon} \frac{\partial \epsilon}{\partial z}+\left(\alpha \frac{\partial \kappa}{\partial \epsilon^{\prime}}+\kappa T_{\lambda}\right) \frac{\partial^{2} \epsilon}{\partial z^{2}}
$$

We guess that there is a wave of the form

$$
\begin{aligned}
\epsilon(z, t) & =\epsilon_{0}+\delta(z, t) \\
& =\epsilon_{0}+\delta_{0} e^{-D k^{2} t} e^{i k(z-v t)} .
\end{aligned}
$$

Putting in the trial solution gives

$$
C T_{\lambda}\left(-D k^{2}-i k v\right)=\alpha \frac{\partial \kappa}{\partial \epsilon}(i k)+\left(\alpha \frac{\partial \kappa}{\partial \epsilon^{\prime}}+\kappa T_{\lambda}\right)(i k)^{2}
$$

From the real parts, the attenuation is given by

$$
D=\frac{\kappa}{C}+\frac{\alpha}{C T_{\lambda}} \frac{\partial \kappa}{\partial \epsilon^{\prime}}
$$

(Note, if we had ignored the $\epsilon^{\prime}$ dependence of $\kappa$ we would have gotten $D=\frac{\kappa}{C}$.) From the imaginary parts, the velocity is given by

$$
v=-\frac{\alpha}{C T_{\lambda}} \frac{\partial \kappa}{\partial \epsilon}
$$

It is important to note that since $\frac{\partial \kappa}{\partial \epsilon}<0, v$ is positive and the wave travels upstream against the heat flux that maintains the SOC state.

We can transform the expression for the velocity into a more easily measured quantity by using the simple expression for the SOC state thermal conductivity, $\kappa=\frac{Q_{\mathrm{SOC}}}{\alpha}$, where $Q_{\mathrm{SOC}}$ is the nominal heat flux of the SOC state. This is, of course, not strictly true in the case where there is a traveling wave, but any correction will be small because $T_{\lambda} \frac{\partial \epsilon}{\partial z} \ll \alpha$. Taking the derivative gives

$$
\frac{\partial \kappa}{\partial \epsilon}=\frac{T_{\lambda}}{\alpha} \frac{\partial Q_{\mathrm{SOC}}}{\partial T}
$$

where $\frac{\partial Q_{\mathrm{SOC}}}{\partial T}$ is easily extracted by measuring $T_{\mathrm{SOC}}$ as a function of $Q$. Substituting into eq. (1.13) gives an alternate expression for the velocity

$$
v=-\frac{1}{C} \frac{\partial Q_{\mathrm{SOC}}}{\partial T}
$$

We cannot explore the thermal conductivity as a function of the temperature gradient because the gradient is fixed by gravity. Instead, we will need to make additional assumptions to make any headway on the attenuation given by eq. (1.12). We borrow from the model of Moeur et al. and 
assume the thermal conductivity is of the form

$$
\kappa=\kappa_{0} \theta^{-x}
$$

where

$$
\theta=\epsilon+\left(Q / Q_{0}\right)^{y} .
$$

Remembering that the heat flux is given by

$$
Q=\kappa \frac{\partial T}{\partial z}=\kappa\left(\alpha+T_{\lambda} \frac{\partial \epsilon}{\partial z}\right),
$$

we take the derivative with respect to $\epsilon^{\prime}$ to get

$$
\begin{aligned}
\frac{\partial \kappa}{\partial \epsilon^{\prime}} & =-\frac{x \kappa}{\theta} \frac{\partial \theta}{\partial \epsilon^{\prime}} \\
& =-\frac{x \kappa}{\theta} \frac{y}{Q}\left(\frac{Q}{Q_{0}}\right)^{y} \frac{\partial Q}{\partial \epsilon^{\prime}} \\
& =-\frac{x y}{\theta \alpha}\left(\frac{Q}{Q_{0}}\right)^{y}\left(\alpha \frac{\partial \kappa}{\partial \epsilon^{\prime}}+T_{\lambda} \frac{\partial \epsilon}{\partial z} \frac{\partial \kappa}{\partial \epsilon^{\prime}}+T_{\lambda} \kappa\right) .
\end{aligned}
$$

We drop the second term in the rightmost grouping because it is much smaller than the first, given $T_{\lambda} \frac{\partial \epsilon}{\partial z} \ll \alpha$. This gives us

$$
\frac{\partial \kappa}{\partial \epsilon^{\prime}}=-\frac{x y}{\theta}\left(\frac{Q}{Q_{0}}\right)^{y}\left(\frac{\partial \kappa}{\partial \epsilon^{\prime}}+\frac{T_{\lambda} \kappa}{\alpha}\right) .
$$

We solve for $\frac{\partial \kappa}{\partial \epsilon^{\prime}}$ to get

$$
\frac{\partial \kappa}{\partial \epsilon^{\prime}}=-\frac{T_{\lambda} \kappa}{\alpha\left(1+\frac{\theta}{x y}\left(\frac{Q}{Q_{0}}\right)^{-y}\right)} .
$$

This give us a model-dependent expression for the attenuation of

$$
D=\frac{\kappa}{C}\left(1-\frac{1}{1+\frac{\theta}{x y}\left(\frac{Q}{Q_{0}}\right)^{-y}}\right) .
$$

It is worth exploring both the low and high $Q$ limits before we have the data to plug in actual values for $x, y$, and $\theta(Q)$. In the low $Q$ limit, $Q^{-y}$ grows very large and we obtain

$$
\lim _{Q \rightarrow 0} D=\frac{\kappa}{C} \simeq \frac{Q}{\alpha C}
$$

In the high $Q$ limit, $\kappa$ becomes large and therefore $\theta$ is small, giving

$$
\lim _{Q \rightarrow \infty} D=\frac{\kappa}{C} \frac{\theta}{x y}\left(\frac{Q}{Q_{0}}\right)^{-y} .
$$


It is not immediately apparent whether this grows or shrinks as $Q$ becomes large. Therefore, we substitute $\theta=\left(\kappa / \kappa_{0}\right)^{-1 / x}$ and $\kappa=Q / \alpha$ to get

$$
\begin{aligned}
\lim _{Q \rightarrow \infty} D & =\frac{Q}{\alpha C} \frac{1}{x y}\left(\frac{Q}{\alpha \kappa_{0}}\right)^{-1 / x}\left(\frac{Q}{Q_{0}}\right)^{-y} \\
& =\frac{\left(\alpha \kappa_{0}\right)^{1 / x} Q_{0}^{y}}{\alpha C x y} Q^{(1-1 / x-y)}
\end{aligned}
$$

Therefore, if $y+1 / x>1$, the dissipation of the SOC wave will go to zero for high heat flux. Since a previous experiment gave $y=0.813$ and $x=0.663$ [1], we expect little to no attenuation for high heat flux. If, instead, we had ignored the dependence of $\kappa$ on the temperature gradient, we would have predicted that $D=\frac{Q}{\alpha C}$ and that the dissipation would grow larger with heat flux.

Our answers differ from those of the calculation by Sergatskov et al. for two reasons. First, they modeled $\kappa$ as we did in eqs. (1.16) and (1.17). However, they computed $\left(\frac{\partial \kappa}{\partial \epsilon}\right)_{Q}$, treating $Q$ as constant. This gave them a wave speed $v \sim Q^{3.3}$. However, a change in $\kappa$ results in a change in heat flux. Therefore, the derivative cannot be taken keeping the heat flux constant. We avoided this problem by transforming our derivative from $\frac{\partial \kappa}{\partial \epsilon}$ to $\frac{T_{\lambda}}{\alpha} \frac{\partial Q_{\mathrm{SOC}}}{\partial T}$. If we assume, as they did, that

$\epsilon_{\mathrm{SOC}} \sim Q^{0.813}$, we get $v \sim Q^{0.167}$. Second, in the Sergatskov paper, they ignored the dependence of the thermal conductivity $\kappa$ on the gradient in reduced temperature. As noted above, this causes an overestimate in the dissipation, especially for high heat flux. With these modifications, we calculate a very different velocity and attenuation for the SOC wave at all but the lowest heat fluxes.

\subsection{Summary}

Some of the questions brought up in this introduction are the following:

- Is $T_{\mathrm{SOC}}(Q)$ a true critical point?

- Is Haussmann's calculation of $C_{\nabla T}$ accurate?

- Can a treatment of the SOC state thermal conductivity as a function of reduced temperature and its derivative accurately predict the speed and attenuation of the SOC wave?

In order to answer these questions, we have measured $C_{\nabla T}, T_{\mathrm{SOC}}$ vs. heat flux $Q$, and the speed and attenuation of the SOC wave. Before discussing those measurements, it is necessary to describe the experimental apparatus. 


\section{Chapter 2}

\section{Apparatus}

The requirements for doing experiments on the SOC state are similar to those of most other ${ }^{4} \mathrm{He}$ lambda point experiments. We need an experimental platform with a very stable temperature and heat flux at the lambda point. We want to investigate heat fluxes in the range of $30 \mathrm{nW} / \mathrm{cm}^{2}$ to $10 \mu \mathrm{W} / \mathrm{cm}^{2}$. The range in SOC state temperatures of these heat fluxes is only $1 \mu \mathrm{K}$. We therefore need subnanokelvin temperature measurement precision and subnanowatt heat flux stability.

\subsection{Cryogenic System}

A schematic of the dewar and probe insert is presented in Fig 2.1. It is a typical cryostat for this application. The dewar has a waist to give a long hold time between transfers of approximately six days for a relatively short dewar only four feet tall. It is surrounded by a $\mu$-metal shield to screen out magnetic fields. The dewar is attached at the top to an aluminum platform (not pictured), which is supported from the floor by three air legs to reduce vibration. Ironically, the aluminum plate that is so carefully isolated from vibration has three large cavities that act as organ pipes and vibrate sympathetically with the noises in the lab. It was not until my ear happened to be close to one end, and I heard the clear tone, that I discovered the source of some of our vibrational noise. Once the cavities were filled with foam, this vibration was significantly reduced.

The probe insert is composed of a brass top plate, stainless steel support tubes and an aluminum vacuum can. There are copper baffles to limit radiative and convective warming. Just inside the vacuum can there is a $1 \mathrm{~K}$ pot to provide cooling of the experiment below $4 \mathrm{~K}$. It has a highimpedance inlet that passes through a filter. In addition, there is a pump line for the vacuum can, a pump line for the $1 \mathrm{~K}$ pot and conduits for the four SQUID leads.

The probe has 56 manganin wires arranged in twisted pairs. At the top of the probe, these wires pass through a custom filter box. This box has two chambers with commercial pi-filters $(3 \mathrm{~dB}$ cutoff at $0.8 \mathrm{MHz}$ ) mounted on the dividing wall. This prevents the RF electromagnetic noise from the unfiltered wires from being radiated and then picked up by the filtered lines. The wires pass 


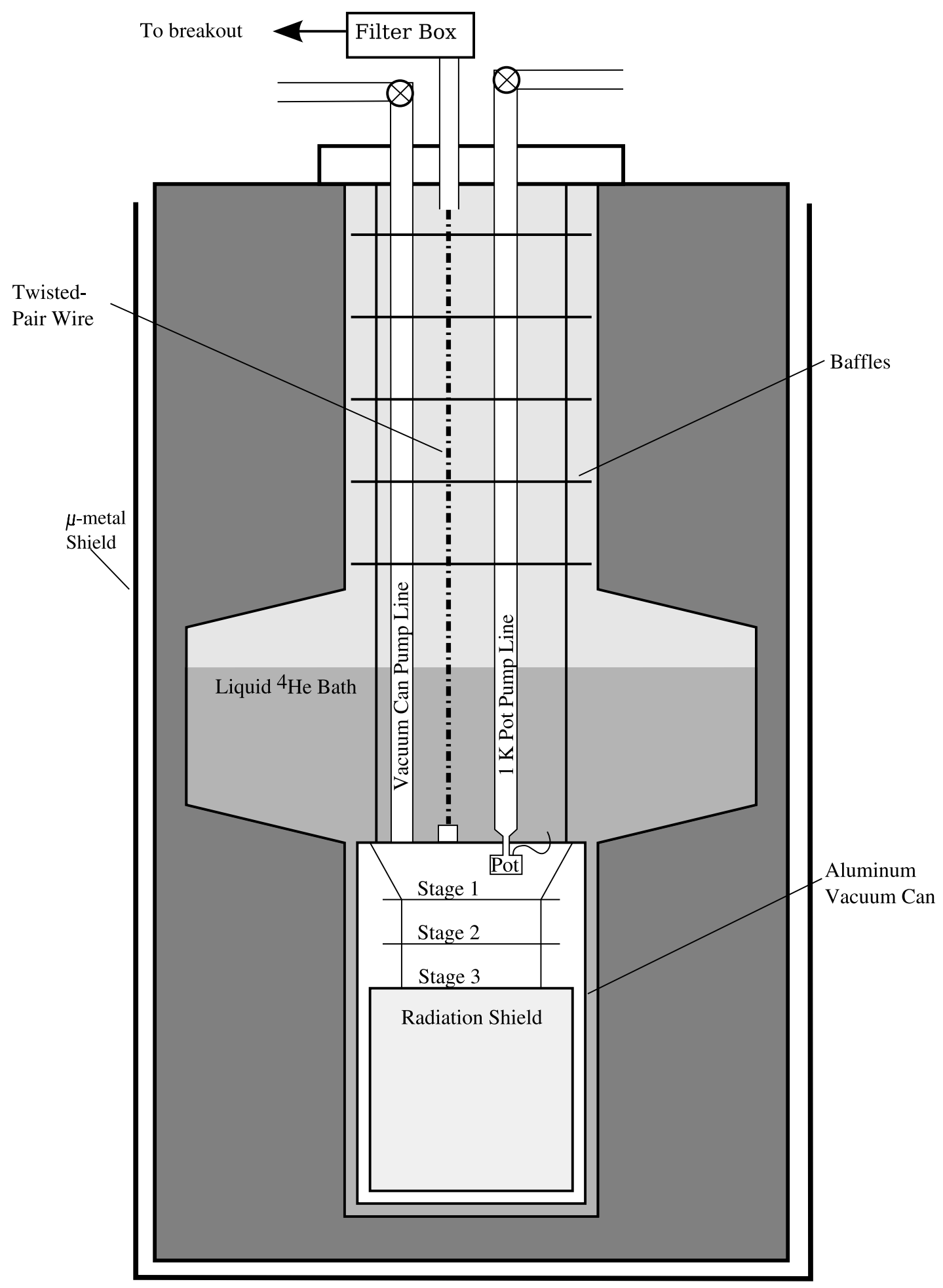

Figure 2.1. Schematic of the cryostat. 
from the filter box down the probe to a glass-sealed connector at the top of the vacuum can. All inlets to the vacuum can are hermetically sealed with indium. From there, the wires go down to their respective stages, getting heat-sunk at each stage they pass. In order to reduce the heat load, the wires are switched to $\mathrm{CuNi}$-clad superconducting $\mathrm{NbTi}$ wire as they travel from stage 3 to the experiment stage.

\subsection{Thermometry}

Two types of thermometers are used in this experiment: Germanium Resistance Thermometers (GRTs) and High Resolution Thermometers (HRTs). The GRTs are used for coarse measurements as well as for a calibrated measure of temperature. We use the calibration charts provided with the GRTs by LakeShore.

An HRT is formed with a paramagnetic material, operating very close to its Curie temperature, that is inductively coupled to a superconducting loop that is attached to a SQUID. The paramagnetic material is subjected to a very stable magnetic field and shielded from any external magnetic disturbances by a superconducting shield. The SQUID then measures the magnetization of the material, which is strongly temperature dependent. The noise of these thermometers is frequently better than $1 \mathrm{nK} / \sqrt{\mathrm{Hz}}[12]$.

Our HRTs are inherited prototypes from the MISTE project [13]. The paramagnetic material for at least one of the HRTs is PdMn, but the specifics of the materials and concentration ratios are not known to us. The sensor is composed of the sensing material wrapped with approximately 20 loops of $\mathrm{NbTi}$ wire, sandwiched between two permanent magnets, and shielded by a Nb housing. The superconducting leads, shielded by a NbTi capillary, travel from the sensor to the SQUID that is mounted on the SQUID stage, shown in fig. 2.2. We have three RF SQUIDs and one DC SQUID providing a total of four HRTs.

\subsection{Auxiliary Stages}

A schematic of the thermal stages inside the vacuum can is shown in fig. 2.2. Stages 1,2, and 3 serve to provide a stable temperature platform for the experiment. The thermal resistance between the stages and the heat capacity of each stage provide a passive filter to temperature oscillations and thermal noise. Stages 2 and 3 each have a thermal ballast of compressed helium to increase their heat capacity and improve the filtering. In addition, each of these stages was actively controlled with PID control algorithms. The SQUID stage serves as the mounting location for the four SQUIDs that control the HRTs. While this stage has a GRT and heater, it was not temperature controlled.

Stages 1 and 2 are each outfitted with a GRT and a heater for temperature control. Stage 3 has 


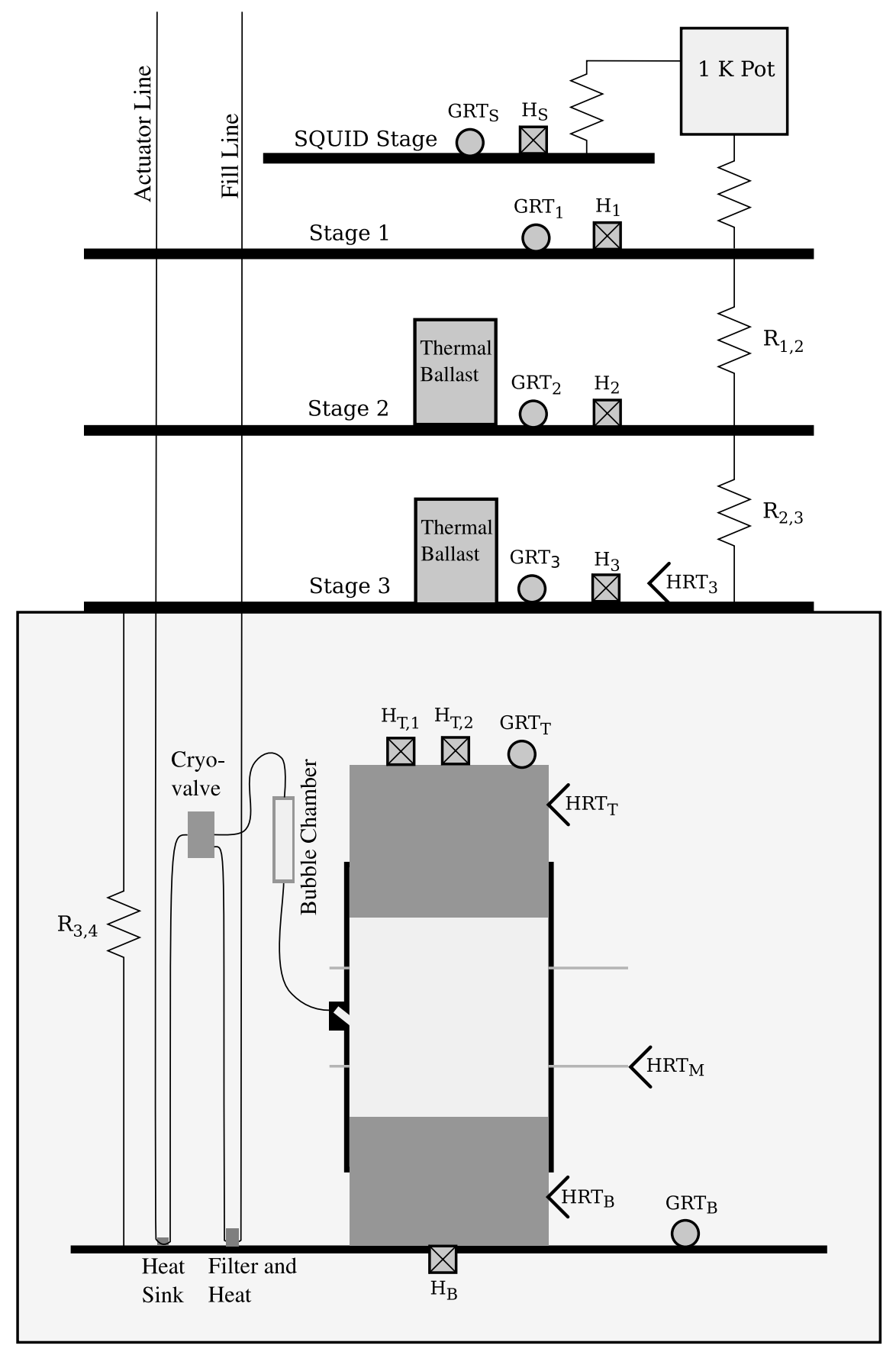

Figure 2.2. Schematic detail of the stages. 


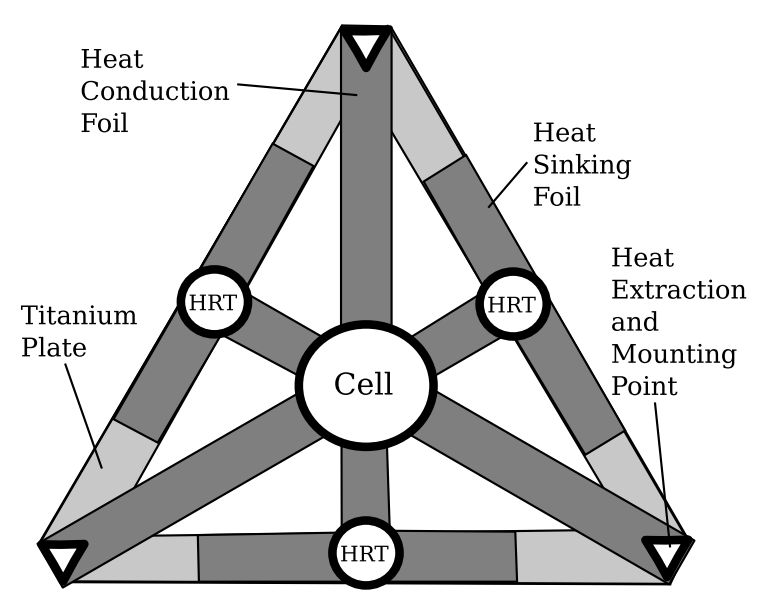

Figure 2.3. Schematic of the mounting platform and the placement of the copper foils, HRTs, and cell.

an HRT in addition to the GRT and heater. During all data runs, the stage was thermally controlled with the HRT to a temperature precision of better than $100 \mathrm{nK}$. The gold-plated aluminum radiation stage is mounted and thermally anchored to this stage. A more sophisticated experiment would have the shield stage outside of the thermal path so that it could be maintained at the same temperature as the experiment. In our setup, the radiation stage was usually regulated approximately $10 \mathrm{mK}$ colder than the experiment.

\subsection{Cell Stage}

The cell stage, which houses the experimental sample of ${ }^{4} \mathrm{He}$, is thermally isolated from stage 3 by a large $(>1000 \mathrm{~K} / \mathrm{W})$ thermal resistance and shielded from thermal radiation by a gold-plated aluminum radiation shield.

\subsubsection{Mounting Platform}

The mounting platform for the experiment stage is a titanium plate. Titanium was chosen for its strength and low heat capacity relative to stainless steel. Annealed copper foils are attached to the top of the mounting plate to increase the thermal conductivity. They are drawn schematically in fig. 2.3. There are two types of foils, each intended to serve a different purpose. There are three foils to conduct the heat flux from the cell bottom plate to the mounting legs where the heat is extracted. Three other foils are used to create heat sinking points that are at the same temperature as the cell bottom plate. Unfortunately, as will be seen in the next chapter, too much of the heat goes through the second type of foil, which causes heat leak problems. 
The mounting platform is attached to stage 3 by three titanium legs. The titanium tubing wall thickness was 0.025 ", but the half of the tubing closer to the cell was reamed to approximately 0.012". To ensure the proper thermal resistance between stage 3 and the cell stage, approximately $4000 \mathrm{~K} / \mathrm{W}$, each leg is coiled with $30 \mathrm{~cm}$ of 0.012 " copper wire.

All nuts and bolts on the mounting platform are titanium to reduce the heat capacity. Dow Corning 340 silicone heat sinking compound was used to reduce the thermal contact resistance of all joints.

\subsubsection{Cell Construction}

The experimental cell is composed of two Oxygen-Free High-Conductivity (OFHC) copper endplates with an insulating Vespel sidewall. The copper endplates are $2.286 \mathrm{~cm}$ in diameter at their face and are $2 \mathrm{~cm}$ and $3.3 \mathrm{~cm}$ tall for the bottom and top endplates, respectively. The endplates were annealed to further increase their thermal conductivity. The faces were polished to a mirror finish with $0.3 \mu \mathrm{m}$ alumina powder and the entire endplate was gold plated. The drawings for the two endplates and the rest of the experiment stage are included in App. A.

The sidewall was made from three $0.64 \mathrm{~mm}$ thick Vespel sleeves with $2.301 \mathrm{~cm}$ inner diameter (ID) and two OFHC copper foils with holes of the same ID. The sleeves and foils were assembled on a Teflon mandril with a copper foil between each pair of sleeves. One copper foil is $165 \mu \mathrm{m}$ thick and the other is $90 \mu \mathrm{m}$ thick. The assembly was glued together using TraCon epoxy (TraBond BA-2151) to form one sleeve with two penetrating foils. A Dremel tool was used to remove excess epoxy that prevented the copper foils from penetrating all the way inside.

The copper endplates were epoxied into the Vespel sidewall sleeve. Care was taken to ensure that the gap between the top endplate and the sidewall was filled with epoxy, since there is a heat flux dependent Kapitza resistance when helium is allowed to penetrate between the endplate and the sidewall $[14,15]$. However, because the bottom endplate was epoxied last, we were unable to examine its gap.

From caliper measurements, there is an interior cell height of $2.585 \pm 0.005 \mathrm{~cm}$ at room temperature. From the cell drawings, the interior diameter of the cell is $2.301 \pm 0.005 \mathrm{~cm}$. This gives an interior volume of $10.75 \pm 0.05 \mathrm{~cm}$. The differential contraction of Vespel from room temperature to experimental temperatures is $\Delta L / L=5.1 \times 10^{-3}$ [16]. This gives a cryogenic volume of

$$
V=10.59 \pm 0.05 \mathrm{~cm}^{3} .
$$




\subsubsection{Fill Line and Bubble Chamber}

A $0.005 "$ ID capillary fill line is epoxied into the hole on the Vespel sidewall. The hole in the Vespel starts at a larger diameter, $0.2 \mathrm{~cm}$, in the hopes of maintaining a superfluid link between the main cell volume and the bubble chamber, despite the thin capillary. The capillary then connects to the bubble chamber, which is mounted higher than the main volume of helium in order to gravitationally aid bubble cavitation. The bubble chamber, with an internal height of $1.35 \mathrm{~cm}$ and diameter of $0.318 \mathrm{~cm}$, has a volume of $0.107 \mathrm{~cm}^{3}$. This was designed to accommodate up to a $1 \%$ helium vapor bubble in order to maintain the helium sample at SVP. The bubble chamber is attached to a high thermal resistance, $\approx 10^{6} \mathrm{~K} / \mathrm{W}$, Vespel holder, which is attached to a heat-sinking foil on the mounting plate.

\subsubsection{Cryo-Valve and ${ }^{3}$ He Actuation System}

The cryo-valve in this experiment was designed by the University of New Mexico for the now canceled NASA flight experiment DYNAMX. Its advantages are that it has a very small mass, has a tiny dead volume, and is available for purchase fully assembled from EMD Machining. Its major disadvantage is that it is a normally open valve that requires a very large closing pressure, on the order of 600 psi. At such high pressures, ${ }^{4} \mathrm{He}$ is a solid at our experimental temperatures, so this valve is designed to use ${ }^{3} \mathrm{He}$ as the actuating medium.

The ${ }^{3} \mathrm{He}$ was concentrated in a charcoal trap cooled in a dewar of ${ }^{4} \mathrm{He}$. Upon warming, the proper pressure to close the valve was reached, and the actuation line was isolated. Unfortunately, with each transfer of helium to cool our experiment, the actuation line near the top of the probe insert would cool sufficiently to cause the total actuator pressure to fall from its nominal value of 580 psi to $420 \mathrm{psi}$; this is despite having a $4 \mathrm{~cm}^{3}$ ballast volume that we included on the room temperature end of the actuator line. We recommend a larger ballast volume or thinner actuator line to reduce this effect. However, due to the hysteretic nature of these valve's opening and closing, the low pressure was not sufficient to cause our valve to open, but it may have contributed to the small fill-line leak we experienced.

The cryo-valve is mounted on the same Vespel holder as the bubble chamber. The fill line and actuator capillaries first travel down to the heat sinking foil on the mounting platform where they are heat sunk. Here, the fill line has a filter to ensure that no foreign matter gets into the cryovalve. The two capillaries then go up, being heat sunk on each stage before heading up to room temperature.

\subsubsection{HRT Mounting}

The three HRTs on the experiment stage are mounted in Vespel holders. These holders have very thin walls, $\approx 0.005 "$, to ensure a very high thermal resistance $\left(R>10^{6} \mathrm{~K} / \mathrm{W}\right)$ and therefore a very 
low heat leak. Each Vespel holder is mounted on the heat sinking foil of the mounting platform see fig. 2.3. The NbTi capillary leaving each HRT is also heat sunk to the same foil, as well as to stage 3, before heading up to the SQUID stage.

Each of the self-contained HRTs has a copper protrusion for thermal anchoring. These tabs are indium soldered to an annealed OFHC copper bar. Each bar travels to its thermal mounting point (either a sidewall foil or copper endplate) where an indium to indium pressure joint is formed. The HRT that is attached to the DC SQUID is mounted to the top endplate. Two RF SQUIDS are used for the HRTs on the bottom endplate and the lower of the two penetrating sidewall foils.

\subsubsection{GRT Mounting}

The GRTs, which are cylindrical, were slid into holes in copper mounts with G.E. Varnish to ensure good thermal contact. One GRT is attached to the cell top plate and the other is attached to one of the heat sinking foils on the mounting plate. Again, Dow Corning heat sinking compound was used for good thermal contact.

\subsubsection{Heaters}

Commercial surface-mount metallic-film resistors are used as heaters on the cell. A small piece of cigarette paper was stuck to the heater location with GE Varnish for electrical isolation. The heater was then stuck to the paper with GE varnish and held down with a small pressure until it dried.

Two four-wire heaters are mounted on the top endplate, and one four-wire heater is attached to the bottom endplate. A two-wire heater is mounted on the bubble chamber to aid bubble nucleation, and another one is placed on the copper bar connecting the HRT to the penetrating sidewall foil for sidewall heating diagnosis. All heaters, whether they are two- or four-wire heaters at the room temperature end, have two superconducting leads from stage 3 down to the cell. The leads are heat sunk to the heater location, as well as to a heat sinking foil on the mounting plate.

\subsection{Data Acquisition and Experiment Control}

\subsubsection{Cryo-Probe Inputs and Outputs}

A shielded cable of twisted pairs connects the filter box at the top of the cryo-probe to a breakout box. This breakout box has connectors for each of the heaters and GRTs. The only other outputs from the probe are the leads for the four HRTs. Three of the HRTs are connected to RF SQUIDs and are read with Quantum Design RF SQUID controllers. The last HRT is connected to a DC SQUID, which is read by a Star Cryo Controller. This SQUID was manufactured by Quantum Design, and initially we used a Quantum Design controller. However, the output of the Quantum 


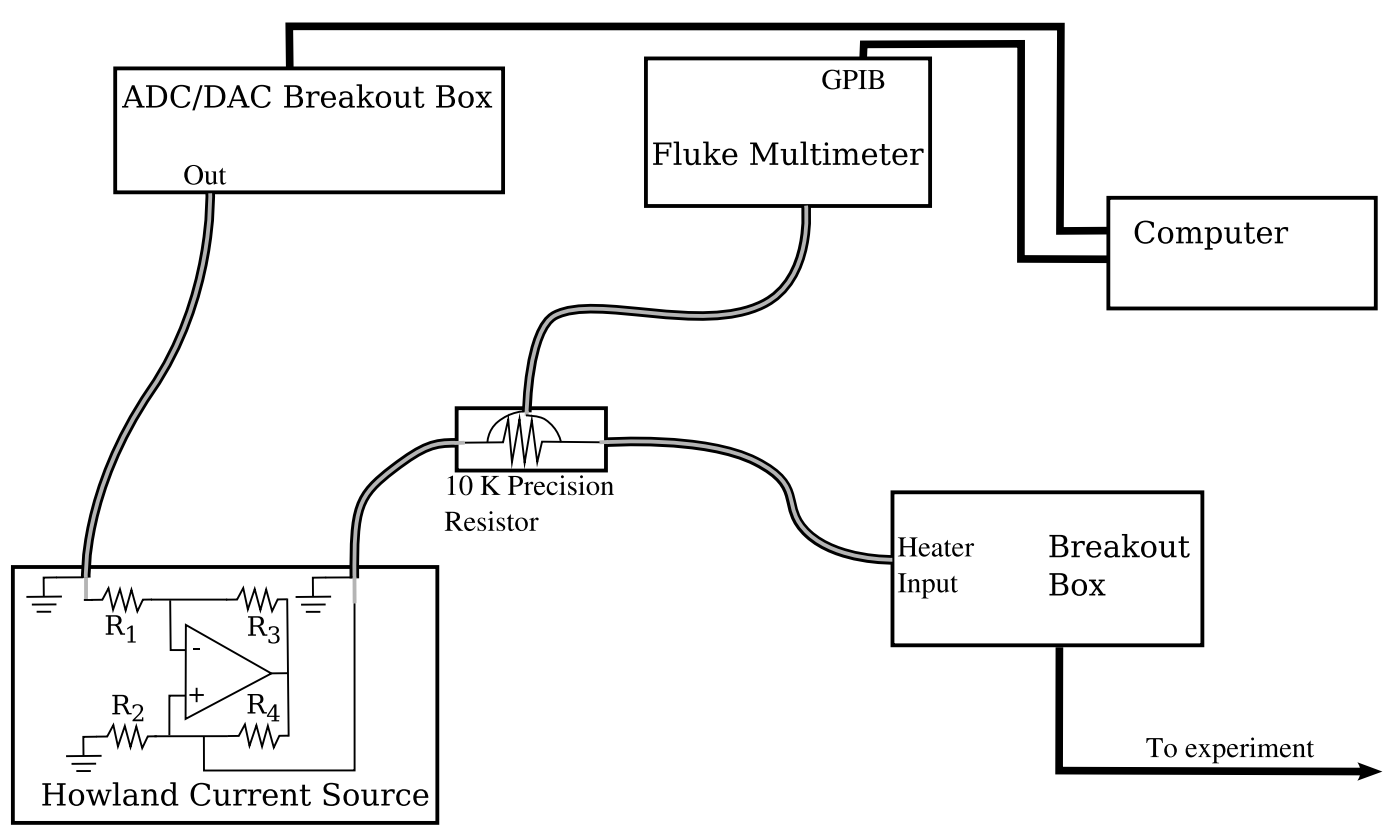

Figure 2.4. Schematic of the heater control.

Design controller was slightly nonlinear for reasons we could not determine and the Star Cryo is immune to this effect. Therefore, the Star Cryo is used in this experiment.

\subsubsection{Data Acquisition and Control}

The heaters and GRTs on stage 1 and 2 are controlled with a bridge, lock-in amplifier and a temperature controller. The resistances are measured with the combination of a Stanford SR510 lock-in amplifier and custom resistance bridge. A resistance decade box is placed opposite the sensor GRT in the bridge, so that any resistance could be dialed up. The voltage across the bridge is input to a Linear Research LR-130 temperature controller whose output is fed to a heater.

A Linear Research LR-700 Resistance Bridge is used to read the GRT resistance of whichever sensor is of interest for a particular experiment. During normal procedures, the LR-700 monitors either the cell top or cell bottom GRT in order to make sure the cell does not get warm enough to rupture. If the cell were to warm past a preset limit, then the experimental control programs would turn off all heating to the cell.

The HRT controllers output a voltage proportional to the flux. The RF controllers have two outputs, one filtered and one unfiltered. The filter is set to $100 \mathrm{~Hz}$ and both outputs are input into an ADC connected to the computer. The DC controller has no internal filter so the output is split and one end is run through a simple RC low-pass filter with a $3 \mathrm{~dB}$ point of $100 \mathrm{~Hz}$. Again, both the filtered and unfiltered inputs are input into the ADC.

The heater on stage 3 is directly controlled with the Digital to Analog Converter (DAC) in the 
computer. The voltage is applied through a large series resistor to limit the current. The heaters on the experiment stage, however, need to be powered in a careful fashion to precisely account for the heat applied. The schematic for this setup is shown in fig. 2.4. The DAC output is input into a Howland current source circuit. We have two different types of channels available. The first uses precision resistor values $R_{1}=R_{2}=500 \mathrm{k} \Omega$ and $R_{3}=R_{4}=50 \mathrm{k} \Omega$ and gives $20 \mu \mathrm{A}$ at 10 volts. The second uses precision resistor values $R_{1}=R_{2}=100 \mathrm{k} \Omega$ and $R_{3}=R_{4}=10 \mathrm{k} \Omega$ and gives $100 \mu \mathrm{A}$ at 10 volts. The current is given by $I=V_{\text {In }} / R_{2}$ and can be set by applying the appropriate voltage using the DAC. The output from the current source is passed through a series $10 \mathrm{k} \Omega$ precision resistor to the appropriate heater. A Fluke 8802 Multimeter measures the voltage across the precision resistor to measure the actual current applied.

In addition we have a Keithley 263 Calibrator/Source that we use for some experiments to apply a fixed current to a heater.

\subsubsection{Computer Inputs and Outputs}

The computer for this experiment uses three National Instruments (NI) cards for input and output. There is a GPIB card, a 64-channel Analog to Digital Converter (ADC) card with two Digital to Analog Converts (DACs) with waveform output capabilities, and a 6 channel DAC card with (unused) digital IO.

The GPIB card is used to retrieve the voltage measurements from the Fluke Multimeters as well as the resistance from the LR-700. The calibration from LakeShore is then used to convert this resistance into a temperature. We also use the GPIB to set the output current of our Keithley Calibrator.

The ADC samples only one channel at a time and rapidly scans over the total number of channels. The ADC reads both the unfiltered and filtered outputs of the four SQUIDS, and perhaps two other voltages, depending on the experiment being performed. It should be noted that the voltage sensed by the ADC exponentially relaxes from the previously sampled channel's voltage to the new channel's voltage. Therefore, depending on how long the ADC samples each channel, there can be a settling time error. We sample each of the ten channels 500 times a second. We find this to be a nice compromise for exceeding the Nyquist frequency of the filtered channel while being slow enough that the settling time does not cause any additional noise in the data.

\subsubsection{Computer Software}

\subsubsection{General Overview}

The experimental software used to run this experiment was written in Java. The algorithms for flux counting and PID control were borrowed from previous software written by Peter Day. There is also 
a small bit of code written in $\mathrm{C}$ to communicate with the NI cards, as there are no Java drivers. The core component of the software runs on the same computer that has these cards. It is responsible for data acquisition, controlling the DACs, logging the data and doing simple PID control. However, most of the code makes use of the Java's Remote Method Invocation (RMI), so algorithms to run specific experiments are often run remotely.

\subsubsection{Flux Counting}

We use a simple flux counting algorithm done entirely in software. The computer takes in 500 samples a second for both the filtered and unfiltered value for each HRT. All flux counting is done on the unfiltered channel because we do not want to smooth over the sharp sawtooth when the SQUID controller resets. Each reset results in the output changing by some integral number of $\Phi_{0}$, the voltage of one flux. A sample that falls outside of $0.2 \Phi_{0}$ of the previous sample is considered "bad" and not included in any averaging. However, if a "good" point (i.e., one that fell within $0.2 \Phi_{0}$ of the previous sample) is more than $0.5 \Phi_{0}$ away from a previous "good" point, then the flux count is adjusted as necessary so that the final value is within $0.5 \Phi_{0}$. This reconstructs the HRT controller output, with its discontinuities resulting from the resets, into a continuous curve.

The filtered value for all "good" points are averaged together to give the required experimental samples per second. This is $2 \mathrm{~Hz}$ during typical time insensitive runs, while for more time critical experiments, we average at a sampling rate of $10 \mathrm{~Hz}$. 



\section{Chapter 3}

\section{Calibration}

\subsection{Thermal Network}

Before introducing any helium into the fill and actuator lines, the thermal network as drawn in fig. 2.2 was measured. We thermally controlled stage 1 as described in sec. 2.5.2. We applied heat to the cell top heater and measured the temperatures of all the stages using the LR-700 once the system reached equilibrium. We did this for a few different heats between 20 and $40 \mu \mathrm{W}$ and found a moderate temperature dependence on the resistances. The values listed below, therefore, represent estimates for the resistance at the temperatures used in the experiment. This gave a total thermal resistance of $7800 \mathrm{~K} / \mathrm{W}$ between the cell and stage 1, which allowed us to apply a maximum heat flux of $13 \mu \mathrm{W} / \mathrm{cm}^{2}$ through the cell and still thermally control all of the stages. $R_{\text {Sidewall }}$ is the thermal resistance from the top endplate to the bottom endplate through the Vespel sidewall.

Table 3.1. Resistances of the thermal network

$\begin{array}{lr}R_{\text {Sidewall }} & 15000 \mathrm{~K} / \mathrm{W} \\ R_{3,4} & 4300 \mathrm{~K} / \mathrm{W} \\ R_{2,3} & 2900 \mathrm{~K} / \mathrm{W} \\ R_{1,2} & 560 \mathrm{~K} / \mathrm{W}\end{array}$

\subsection{Heater Calibrations}

The three heaters that were used for precision work on the cell stage, $\mathrm{H}_{\mathrm{T}, 1}, \mathrm{H}_{\mathrm{T}, 2}$, and $\mathrm{H}_{\mathrm{B}}$, are all wired with four wires. During the calibration, the current sources were attached as shown in fig. 2.4. However, the two other wires of the heater were also attached to another Fluke multimeter. Therefore, we had independent measurements of $I$ and $V$. We took a table of measurements over the entire range of heats used in the experiment for each heater and fit $V$ vs. $I$ with a cubic polynomial. ${ }^{1}$

\footnotetext{
${ }^{1}$ Our measurements in sec. 3.9 make us suspect that the heaters were not well thermally anchored to the cell. This would give significant self-heating and may have caused the nonlinearity that required the cubic fit.
} 


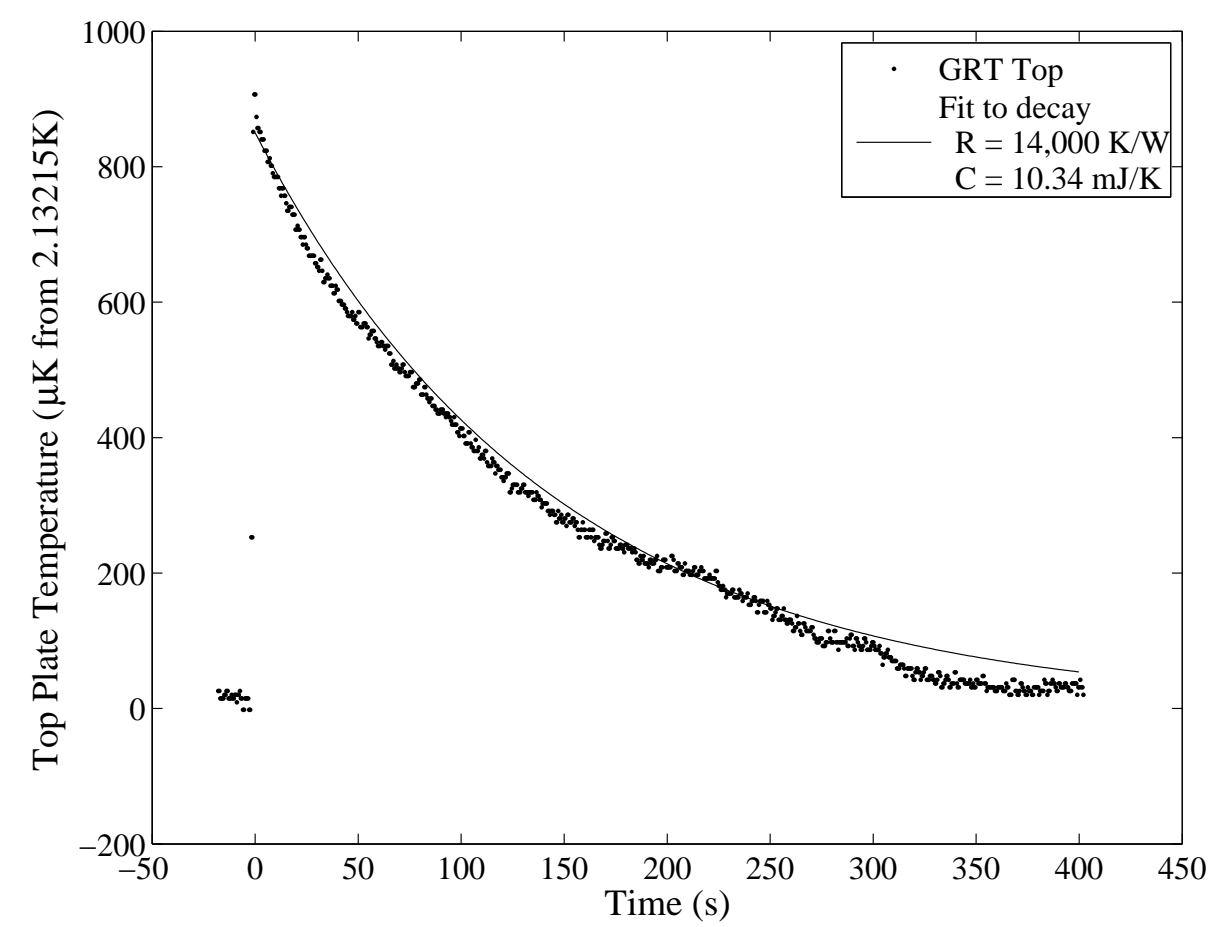

Figure 3.1. Top endplate response to heat pulse.

In table 3.2 we show the calibration values used to compute $V=\mathrm{a} I^{3}+\mathrm{b} I^{2}+\mathrm{R} I+\mathrm{d}$.

Table 3.2. Heater calibrations

\begin{tabular}{lrrrr} 
& \multicolumn{1}{c}{$\mathrm{a}$} & $\mathrm{b}$ & $\mathrm{R}$ & \multicolumn{1}{c}{$\mathrm{d}$} \\
\hline $\mathrm{H}_{\mathrm{T}, 1}$ & 0 & $-1.105210^{6}$ & 10192 & $-2.213510^{-6}$ \\
$\mathrm{H}_{\mathrm{T}, 2}$ & $3.485710^{9}$ & $-1.001810^{6}$ & 10154 & $2.723510^{-5}$ \\
$\mathrm{H}_{\mathrm{B}}$ & $3.15310^{9}$ & $-8.014610^{5}$ & 10161 & $1.658710^{-5}$
\end{tabular}

\subsection{Endplate Heat Capacity}

Since we are doing a high-fidelity heat capacity measurement of the liquid helium in our cell, we need to measure the heat capacity of the empty cell in case we have to subtract it to get the true value for the helium. We measured the heat capacity of each endplate separately. We applied a $9 \mu \mathrm{J}$ pulse to the endplate while measuring the temperature with a GRT. We used the initial temperature step to determine the heat capacity and then monitored the decay back to the original temperature. The results for the cell top are shown in fig. 3.1 and those for the cell bottom in fig. 3.2.

The exponential decays were fit using the just measured end plate heat capacity and the previously measured thermal resistances. For the cell top, we needed to use a sidewall thermal resistance of $14000 \mathrm{~K} / \mathrm{W}$ to get a good fit. For the cell bottom, we used $R=3290 \mathrm{~K} / \mathrm{W}$, the parallel combi- 


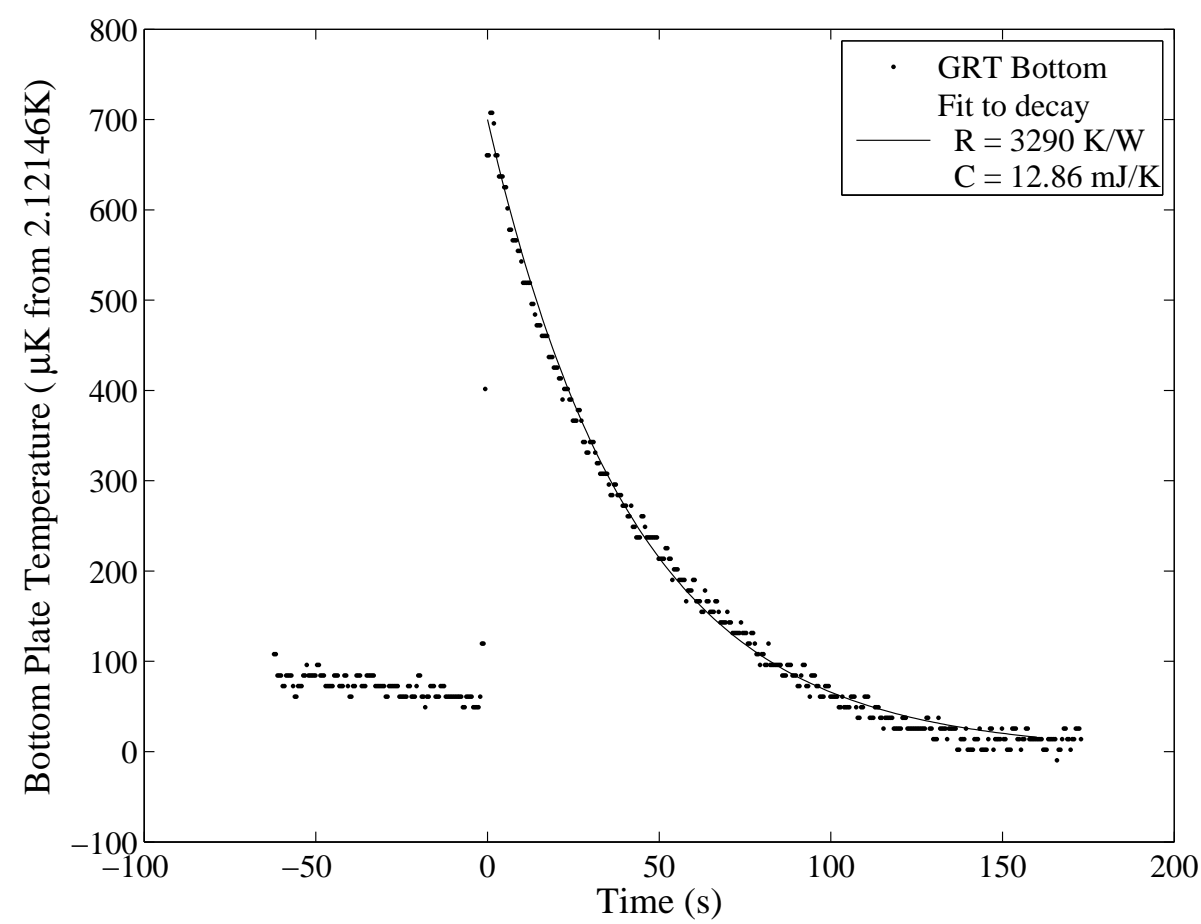

Figure 3.2. Bottom endplate response to heat pulse.

nation of $R_{3,4}=4300 \mathrm{~K} / \mathrm{W}$ and $R_{\text {Sidewall }}=14000 \mathrm{~K} / \mathrm{W}$. The good agreement between fit and data confirms that we have properly measured the heat capacities and thermal resistances. The final results for the heat capacities are given in table 3.3.

Table 3.3. Heat capacities of the endplates

$$
\begin{array}{ll}
\mathrm{C}_{\mathrm{T}} & 10.3 \mathrm{~mJ} / \mathrm{K} \\
\mathrm{C}_{\mathrm{B}} & 12.9 \mathrm{~mJ} / \mathrm{K}
\end{array}
$$

\subsection{Filling the Cell}

While the cell bottom endplate was thermally controlled to $2.7 \mathrm{~K}$, we applied 915 torr of isotopically pure ${ }^{4} \mathrm{He}$ (with a ${ }^{3} \mathrm{He}$ concentration of less than one part in $10^{7}$ ) to the cell fill line and waited for the cell to fill. We then closed the cryo-valve by applying 580 psi of ${ }^{3} \mathrm{He}$ to the actuator line using the concentrator described in sec. 2.4.4.

We removed the remaining helium in the fill line and then pumped on the line with a leak detector to achieve a good vacuum and therefore good thermal isolation. The leak rate settled to approximately $10^{-7} \mathrm{~atm} \mathrm{~cm}^{3} / \mathrm{s}$. Given our 4 month cooldown, the estimated total volume of liquid helium lost due to this leak would be $0.0013 \mathrm{~cm}^{3}$, which is less than a part in 1000 and can be 
safely ignored. In order to maintain a good vacuum, we attached a charcoal cryo-pump to the line, which we periodically cleaned. During transfers, it is possible that, when the actuator pressure fell to $420 \mathrm{psi}$, the leak rate could have increased. However, the total time that the valve was at the reduced pressure over the lifetime of the cooldown was not more than 10 hours.

\subsection{Cavitating the Bubble}

Once the cell was full, we cooled it to the lambda transition. As the helium contracted, we estimate that $0.2 \%$ of the sample was vapor. The molar volume at SVP and $2.7 \mathrm{~K}$ is $27.860 \mathrm{~cm}^{3} / \mathrm{mol}$, and at $T_{\lambda}$ it is $27.386 \mathrm{~cm}^{3} / \mathrm{mol}$ [17]. The compressibility at $2.7 \mathrm{~K}$ is $1.87 \times 10^{-5}$ torr $^{-1}$ [18]. Finally, at $2.7 \mathrm{~K}, \mathrm{SVP}$ is 111 torr [19]. From this we compute the bubble size as

$$
\begin{aligned}
\frac{V_{\text {gas }}}{V_{\text {liq }}} & =\frac{27.86 \mathrm{~cm}^{3} / \mathrm{mol}\left(1-(915 \text { torr }-111 \text { torr }) 1.87 \times 10^{-5} \mathrm{torr}^{-1}\right)-27.386 \mathrm{~cm}^{3} / \mathrm{mol}}{27.386 \mathrm{~cm}^{3} / \mathrm{mol}} \\
& =0.002
\end{aligned}
$$

We can use the cell and bubble chamber volumes computed in sec. 2.4.2, the molar volume of helium, and the size of the bubble to compute the number of moles in our sample.

$$
\begin{aligned}
n & =0.998 \mathrm{~V} / V_{m}=0.998\left(10.59 \mathrm{~cm}^{3}+0.107 \mathrm{~cm}^{3}\right) /\left(27.386 \mathrm{~cm}^{3} / \mathrm{mol}\right) \\
& =0.390 \pm 0.002 \mathrm{~mol}
\end{aligned}
$$

Unfortunately, we could not be sure that the bubble would form inside the bubble chamber. We cooled down to $1.8 \mathrm{~K}$ in order to collapse the bubble and then warmed the cell with the heater attached to the bubble chamber to encourage the bubble to nucleate there. We carefully monitored the temperature during this procedure, hoping to see evidence of bubble collapse or formation as seen by others [20]. However, due to our large volume of helium and lack of thermometer on the bubble chamber, we never saw such a signature.

We do have circumstantial evidence of when the bubble was inside the bubble chamber. On a couple of occasions, we accidentally applied too large a heat to the cell top, causing the cell to warm well past the lambda point. Upon cooling back to the lambda point, and investigating the SOC state, we noticed that the SOC temperature now seemed to be dependent on the temperature at the top of the cell. A set of data showing this effect is shown in fig. 3.3. At $t=0$, the SOC state is established in the bottom of the cell, and a small amount of superfluid still exists at the top (as in profile $\mathbf{A}$ of fig. 1.1). Then, the heat added to the cell top heater is increased and the top warms until normal fluid forms and the SOC/normal interface slowly moves down the cell. When the bubble is in the bubble chamber, there is no observable change in the cell bottom temperature. 
In fig. 3.3(b), however, we can see the SOC temperature falling as the cell top warms. Each time normal fluid enters at the top of the cell (between 100 and $155 \mathrm{~s}$ and between 185 and $220 \mathrm{~s}$ ), the temperature recorded by the bottom thermometer drops. The midplane thermometer (not shown) shows the same drop in temperature.

When a small bubble is at the top of the cell and the liquid helium is in the superfluid phase, the heat flows around the bubble through the superfluid liquid, preventing any temperature or pressure gradient in the bubble. When superflow breaks down, the top endplate warms quickly and evaporates some of the helium which increases the pressure in the bubble. Using the SVP fit from Donnelly [19], the $35000 \mathrm{nK}$ temperature rise that we see in fig. 3.3(a) increases the pressure in the bubble by $0.442 \mathrm{~Pa}$. Given the $1.273 \mu \mathrm{K} / \mathrm{cm}$ gradient in $T_{\lambda}$ and the liquid helium density of $0.14 \mathrm{~g} / \mathrm{cm}^{3}$, the pressure increase causes a $41 \mathrm{nK}$ drop in $T_{\lambda}$. Of course, there is a pressure and temperature gradient across the bubble because the warm helium gas is condensing at the bottom of the bubble which is colder. Therefore, the pressure at the bottom surface of the bubble will be less than the full $0.442 \mathrm{~Pa}$ and the drop in $T_{\lambda}$ will be less than $41 \mathrm{nK}$. We see a drop of $22 \mathrm{nK}$ in fig. 3.3(b).

On the occasions where we observed this phenomenon, we repeated the procedure of cooling down to $1.8 \mathrm{~K}$ and warming with the bubble heater. We then confirmed that the SOC temperature was once again independent of the cell top temperature and therefore assumed that the bubble was properly located in the bubble chamber. 


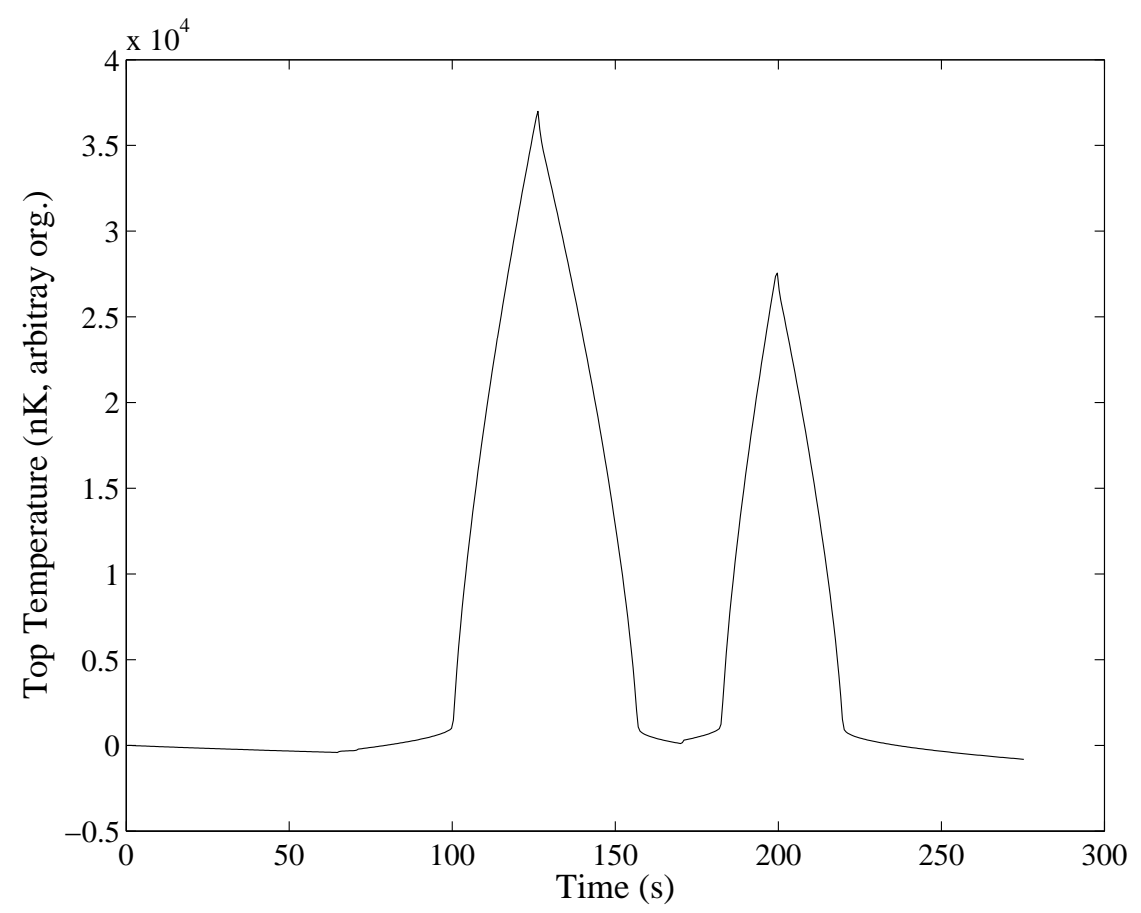

(a) Cell top temperature as heat is added and removed.

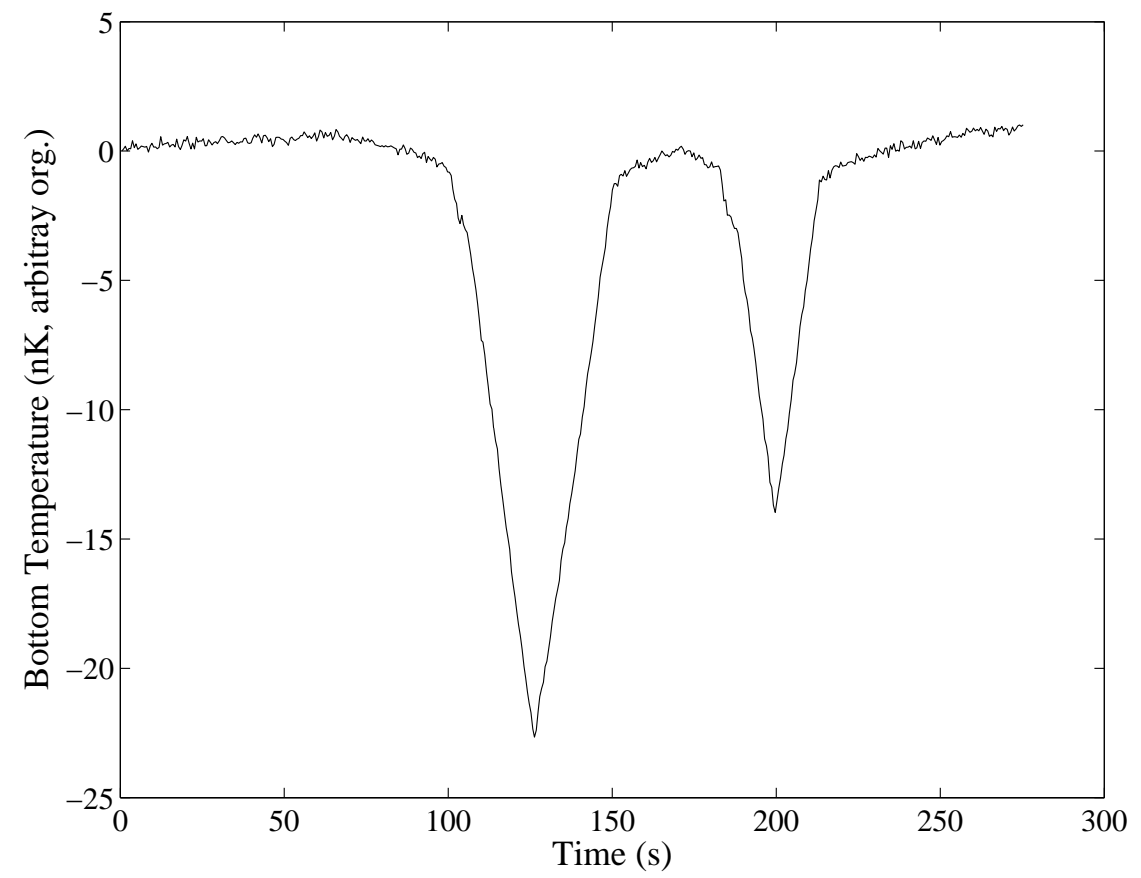

(b) Cell bottom temperature shows the effect on $T_{\lambda}$ of warming and cooling the bubble at the top of the cell.

Figure 3.3. Cell temperature profiles when the bubble is inside the cell and most of the cell helium is in the SOC state. 


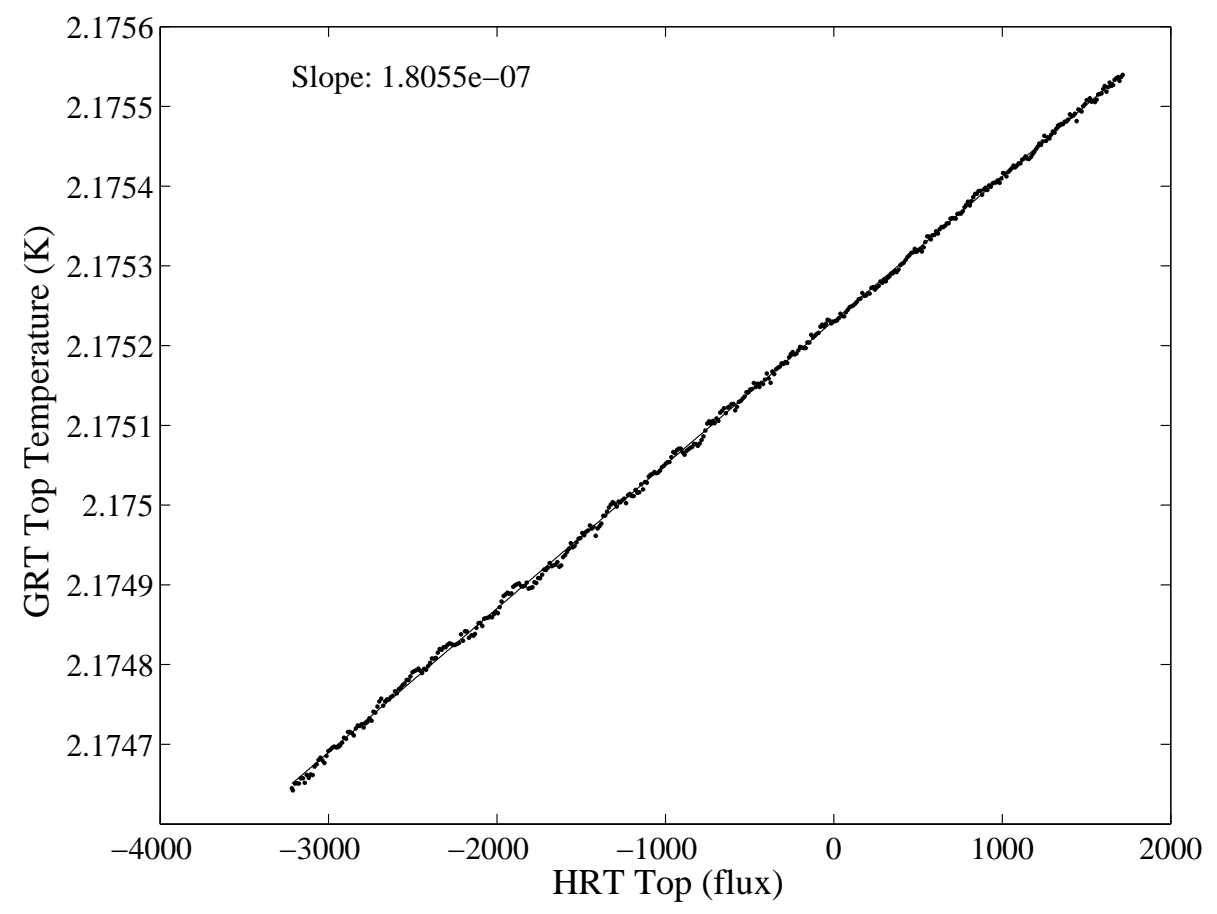

Figure 3.4. Calibration of the top HRT to the top GRT during a ramp down of $1 \mathrm{mK}$ from just below the superfluid transition.

\subsection{HRT Calibration}

With the entire cell just below the superfluid transition, we slowly ramped $(150 \mathrm{nK} / \mathrm{s})$ the cell downwards in temperature about $1 \mathrm{mK}$ while recording the cell top HRT and GRT. The results are shown in fig. 3.4. We fit the top HRT flux to the top GRT temperature to get a calibration for the cell top HRT.

To get calibrations for the other two cell HRTs, we did a slow drift $(0.1 \mathrm{nK} / \mathrm{s})$ of about $350 \mathrm{nK}$ close to $T_{\lambda}$, and calibrated each of these thermometers to the cell top HRT. The results are shown in fig. 3.5 and fig. 3.6. On a previous cooldown, we had calibrated the HRT on stage 3 near $T_{\lambda}$. However, since this HRT now operates over a different temperature range, the calibration is only approximate for the current cooldown. Each HRT does not necessarily have the same paramagnetic material nor permanent magnets of the same strength. Therefore, the calibrations vary widely among the HRTs. The results are summarized in table 3.4.

Table 3.4. HRT calibrations

$\mathrm{HRT}_{\mathrm{T}} \quad 180.6 \pm 0.9 \mathrm{nK} / \Phi_{0}$

$\mathrm{HRT}_{\mathrm{M}} \quad 416.9 \pm 2.1 \mathrm{nK} / \Phi_{0}$

$\mathrm{HRT}_{\mathrm{B}} \quad 81.85 \pm 0.41 \mathrm{nK} / \Phi_{0}$

$\mathrm{HRT}_{3} \quad \approx 3 \mu \mathrm{K} / \Phi_{0}$ 


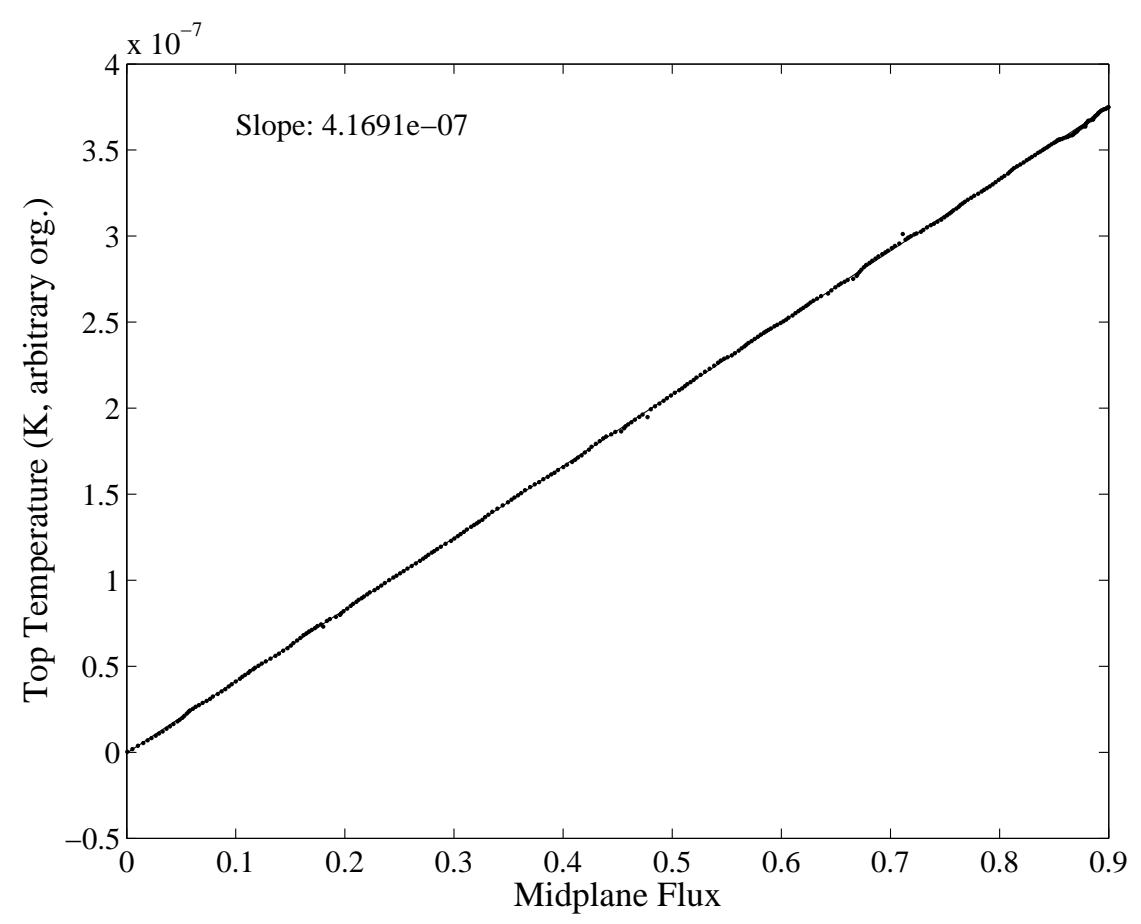

Figure 3.5. Calibration of the midplane HRT to the top HRT during a drift of $350 \mathrm{nK}$.

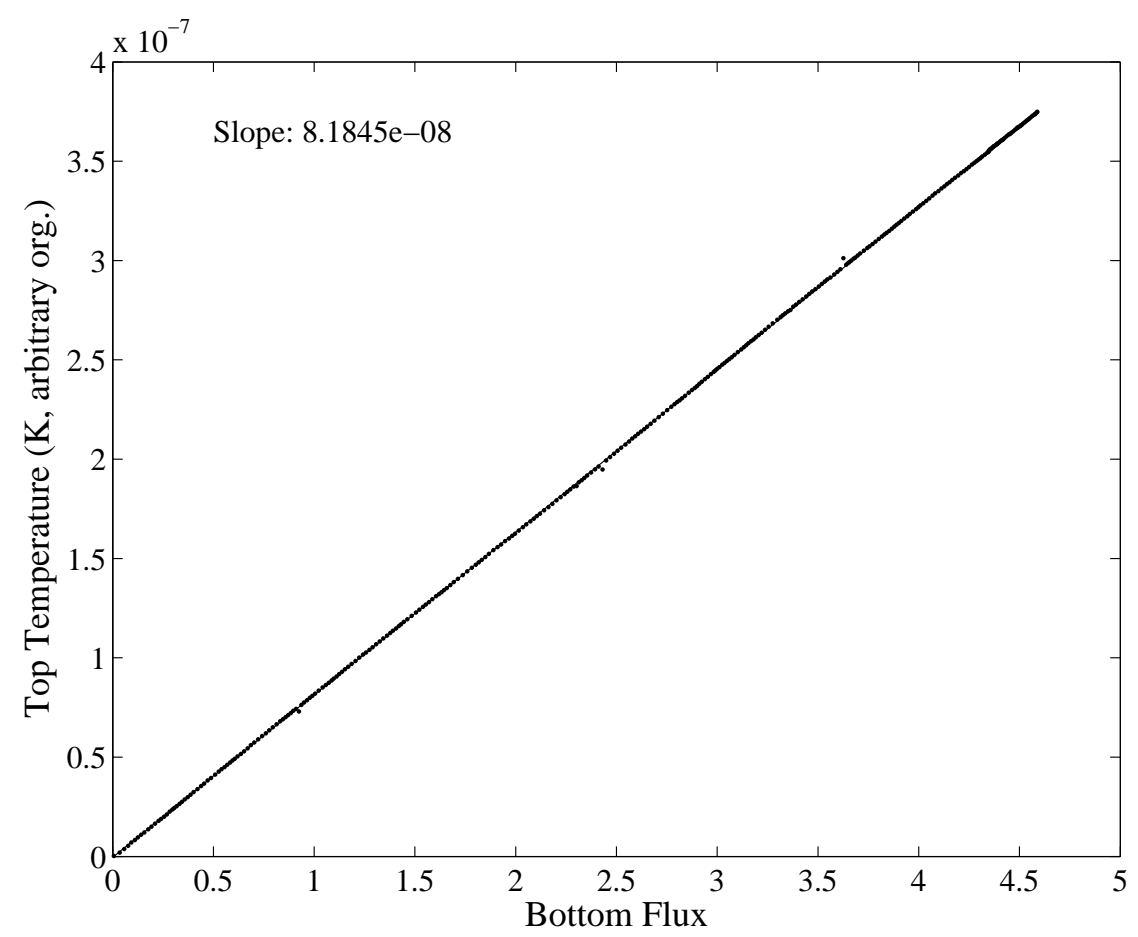

Figure 3.6. Calibration of the bottom HRT to the top HRT during a drift of $350 \mathrm{nK}$. 


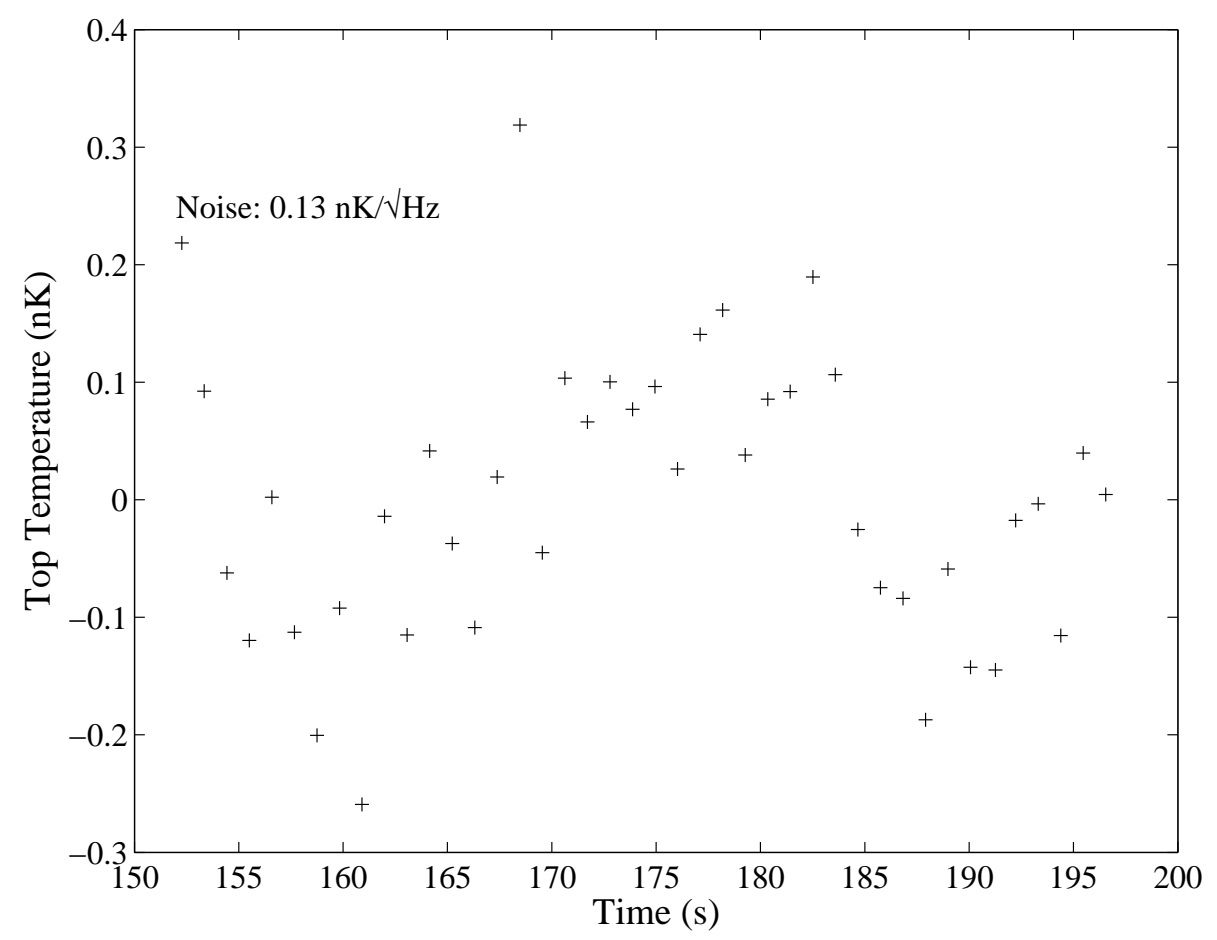

Figure 3.7. Noise of the top HRT.

\subsection{HRT Noise and Drift}

To obtain a value for the HRT noise, we measured each HRT while the helium sample was in the superfluid state with minimal temperature drift. The results, averaged to $1 \mathrm{~Hz}$, are shown in Figs. 3.7, 3.8, and 3.9. The standard deviation from the three data sets was used to compute the thermometer noise given in table 3.5 .

Table 3.5. HRT noise

$\mathrm{HRT}_{\mathrm{T}} \quad 0.13 \mathrm{nK} / \sqrt{\mathrm{Hz}}$

$\mathrm{HRT}_{\mathrm{M}} \quad 0.18 \mathrm{nK} / \sqrt{\mathrm{Hz}}$

$\mathrm{HRT}_{\mathrm{B}} \quad 0.11 \mathrm{nK} / \sqrt{\mathrm{Hz}}$

In analyzing our longer data files, we matched measurements of $T_{\mathrm{SOC}}(Q)$ for the same heat flux $Q$ that were taken many hours apart and found we had a thermometer drift of approximately $0.04 \mathrm{pK} / \mathrm{s}$. This drift is very small and can be ignored for any experiment less than three hours long. 


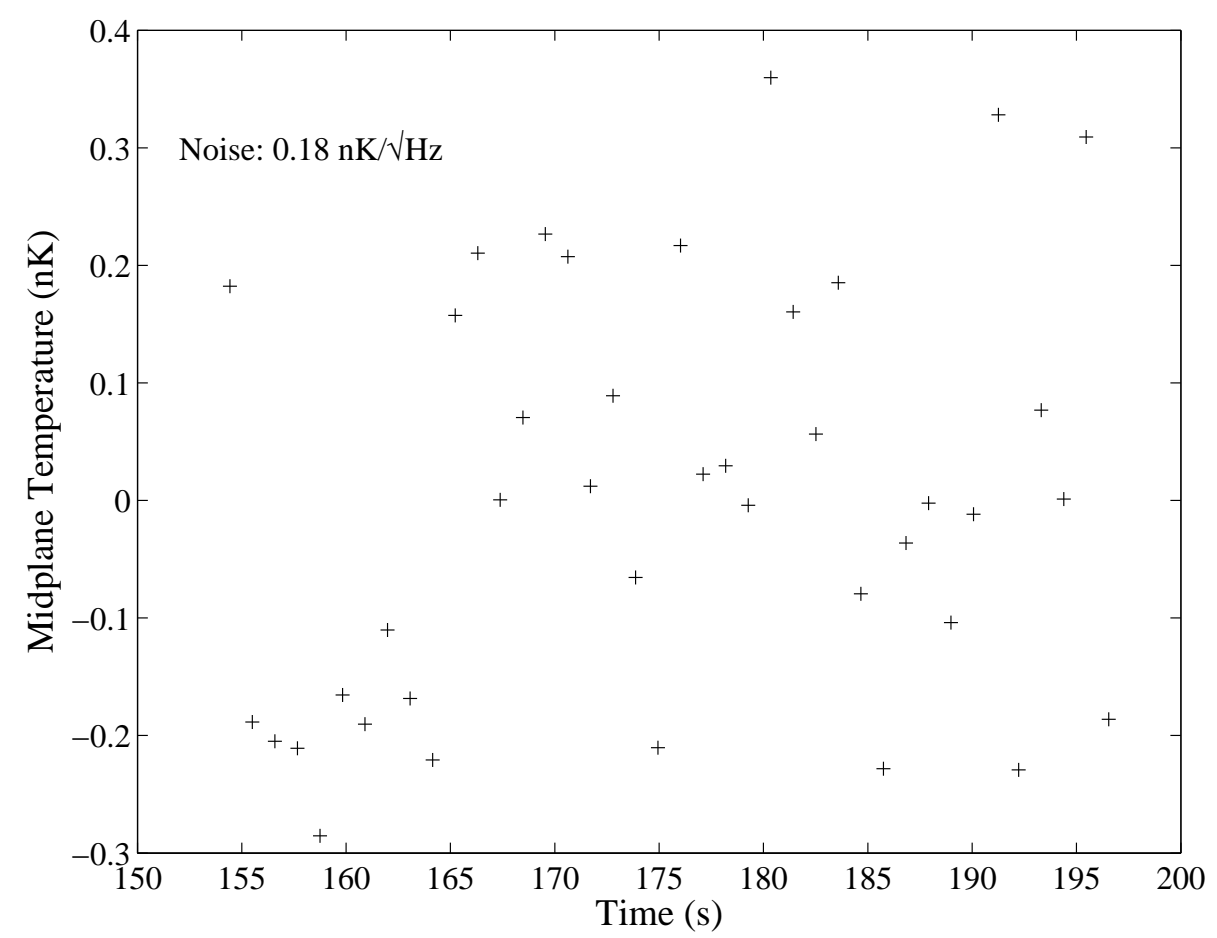

Figure 3.8. Noise of the midplane HRT.

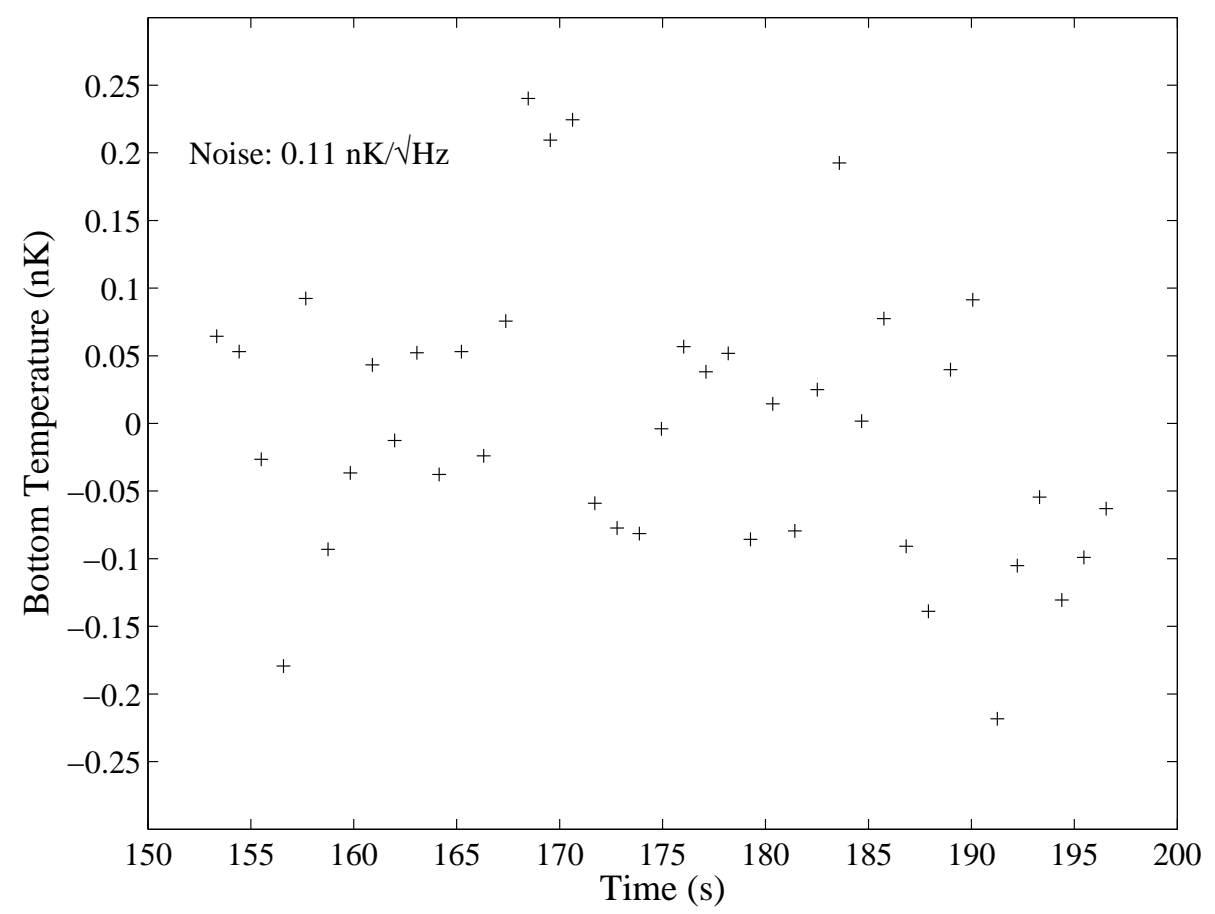

Figure 3.9. Noise of the bottom HRT. 


\subsection{Kapitza Resistance}

Well into the superfluid ( $20 \mu \mathrm{K}$ or so) we took a quick measurement of the regular Kapitza resistance. This is the thermal boundary resistance that arises due to a phonon mismatch across a boundary between two different materials. We lowered the applied heat on the top endplate by about $2 \mu \mathrm{W}$ and increased the heat on the bottom to compensate. The helium temperature stayed roughly the same (see sec. 3.9 for the caveat to this), but the top and bottom endplates changed temperature, allowing us to compute Kapitza resistances across these endplates. They are presented in table 3.6.

Table 3.6. Kapitza resistances

$$
\begin{array}{ll}
R_{\mathrm{K}, \mathrm{T}} & 0.31 \mathrm{~K} / \mathrm{W} \\
R_{\mathrm{K}, \mathrm{B}} & 0.25 \mathrm{~K} / \mathrm{W}
\end{array}
$$

There is another component to the thermal boundary resistance from the endplate into the helium. The superfluid portion of the helium is suppressed near a boundary. Near $T_{\lambda}$, the region over which the superfluid is suppressed gets large and causes a significant increase in the thermal boundary resistance. This has been measured previously in "heat-from-below" configurations [21] and we attempted to measure this singular component of the Kapitza resistance in our "heatfrom-above" configuration. Unfortunately, we measured a heat-flux-dependent resistance. This is caused by having helium in the gap between the endplate and the sidewall [14, 15]. As discussed in sec. 2.4.2, we suspected this might be the case during cell construction. Also, the fact that we see a lower regular Kapitza resistance for the bottom endplate, despite a surface preparation identical to the top, suggests there may be a sidewall gap. ${ }^{2}$

\section{$3.9 \quad$ Heater Response Times}

Our heat capacity measurements, which will be described in the next chapter, are very sensitive to any lag time between applying a voltage to the current source and heat being deposited into the experiment. At one moment in the experiment, the power dissipated in one heater is decreased by the same amount as the increase in another cell heater. If there is no significant lag in the response, or if the lag is equal for both heaters, then no energy will be gained or lost by the helium as this switch between the heaters occurs.

\subsubsection{The Problem}

By measuring the voltage across two wires of the 4-wire heater when a pulse of current was applied, we found that any lag was less than one millisecond. This means there are no problems with the

\footnotetext{
${ }^{2}$ There was no hole for a fill line in either endplate.
} 


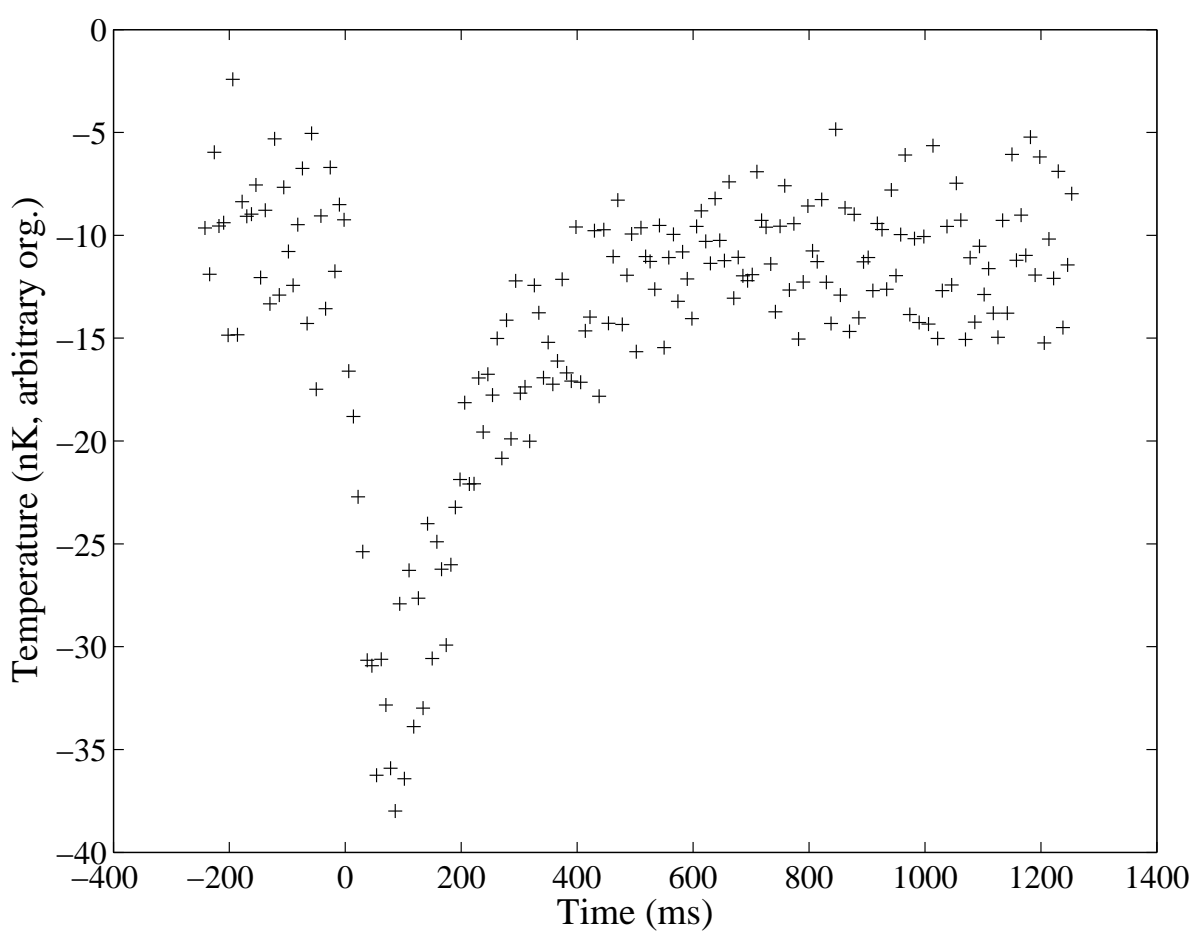

Figure 3.10. Temperature response of the top endplate HRT caused by swapping heat from one heater to another heater with a different time constant.

DAC or current source. However, with the sample entirely in the superfluid, swapping heat from $\mathrm{H}_{\mathrm{T}, 2}$ to $\mathrm{H}_{\mathrm{B}}$ resulted in a small but noticeable change in the helium temperature. The small cell temperature drift was the same before and after the swap.

To confirm that this effect was due to a time lag problem and not some exotic properties of the helium, we repeated the same experiment, except we swapped heat from $\mathrm{H}_{\mathrm{T}, 2}$ to $\mathrm{H}_{\mathrm{T}, 1}$, heaters that are on the same endplate. In fig. 3.10, we see the temperature response of $\mathrm{HRT}_{\mathrm{T}}$ to the swap at $t=0$ with very little data averaging. Obviously, the heater that was reduced responded faster than the heater that was increased. The output is complicated by both the time constant of the copper endplate relaxing to the thermal bath of the helium sample and the time constant of the HRT coming into equilibrium with the endplate. The end result, only seen with more data averaging for lower noise, is that the helium cooled about $1 \mathrm{nK}$. When we reverse the swap, the response is identical, but in the reverse direction. Since we are swapping between two heaters on the same endplate, there is no change in heat flux through the sample, so no helium properties could cause this effect.

We hypothesize that this time constant is caused by the heaters being poorly attached to their locations resulting in an appreciable thermal contact resistance. When a voltage is applied, the heater responds immediately. However, the heater, with its heat capacity and thermal resistance to its mounting point, acts as an RC low-pass filter. Each heater has its own thermal contact 
resistance, so the time constant is different for each one. A further complication is that this time constant appears to change with heat flux. This could be due to either the heater's heat capacity or thermal contact resistances changing as the heater changes temperature.

\subsubsection{The Solution}

Our solution was to calibrate and quantify the size of this error by performing the same experiment where we first saw the effect. We cool well below the superfluid transition and swap the heat from the top heater to the bottom and observe the temperature change of the helium. Using the heat capacity of the helium, it is possible to extract how much energy was lost or gained in the swap. We need to perform this experiment with the same pair of heaters for the same heat fluxes that we use in our heat capacity measurements for the calibrations to be helpful.

To improve the calibrations, the actual procedure worked as follows. For each pair of heat flux values, we swapped back and forth while intentionally delaying the change of the faster heater by a time, $\tau$. We started with small delays and then gradually increased their length. We then plotted the temperature change of the helium vs. the delay. Fig. 3.11 is an example of this. We fit the results to a line. The zero crossing of this line tells us how much time delay is necessary so that no energy is gained or lost. If the faster heater was changed by an amount of heat $A \Delta Q$, where $A$ is the cell cross-sectional area, then the energy $\Delta E$ gained for no delay is $\Delta E=\tau A \Delta Q$. We did not perform this experiment for every pair of heat flux values, but instead changed the heat flux through the cell from 0 to $1 \mu \mathrm{W} / \mathrm{cm}^{2}$, from 1 to $2 \mu \mathrm{W} / \mathrm{cm}^{2}$, from 2 to $3 \mu \mathrm{W} / \mathrm{cm}^{2}$, and so on. We then fit the resulting curve to a parabola (or a line if there were fewer than four points) which allowed us to scale $\Delta E$ for any heat flux swap.

\subsection{Heat Leaks}

Unfortunately, in the design and construction of the experimental cell, we made a miscalculation of the size and arrangement of the copper foils that are placed on the mounting plate. One set of foils was intended to conduct away the heat flux, and the other set of foils was to maintain a heat sinking location close in temperature to the cell bottom endplate. Either through high contact resistance, higher than expected conductivity of titanium, or some other unexpected reason, the temperature of the heat-sinking foils was significantly different from the cell bottom endplate. This gave us relatively large heat leaks (a few nanowatts) through the HRTs and their Vespel mounts and possibly through the fill line as well. 


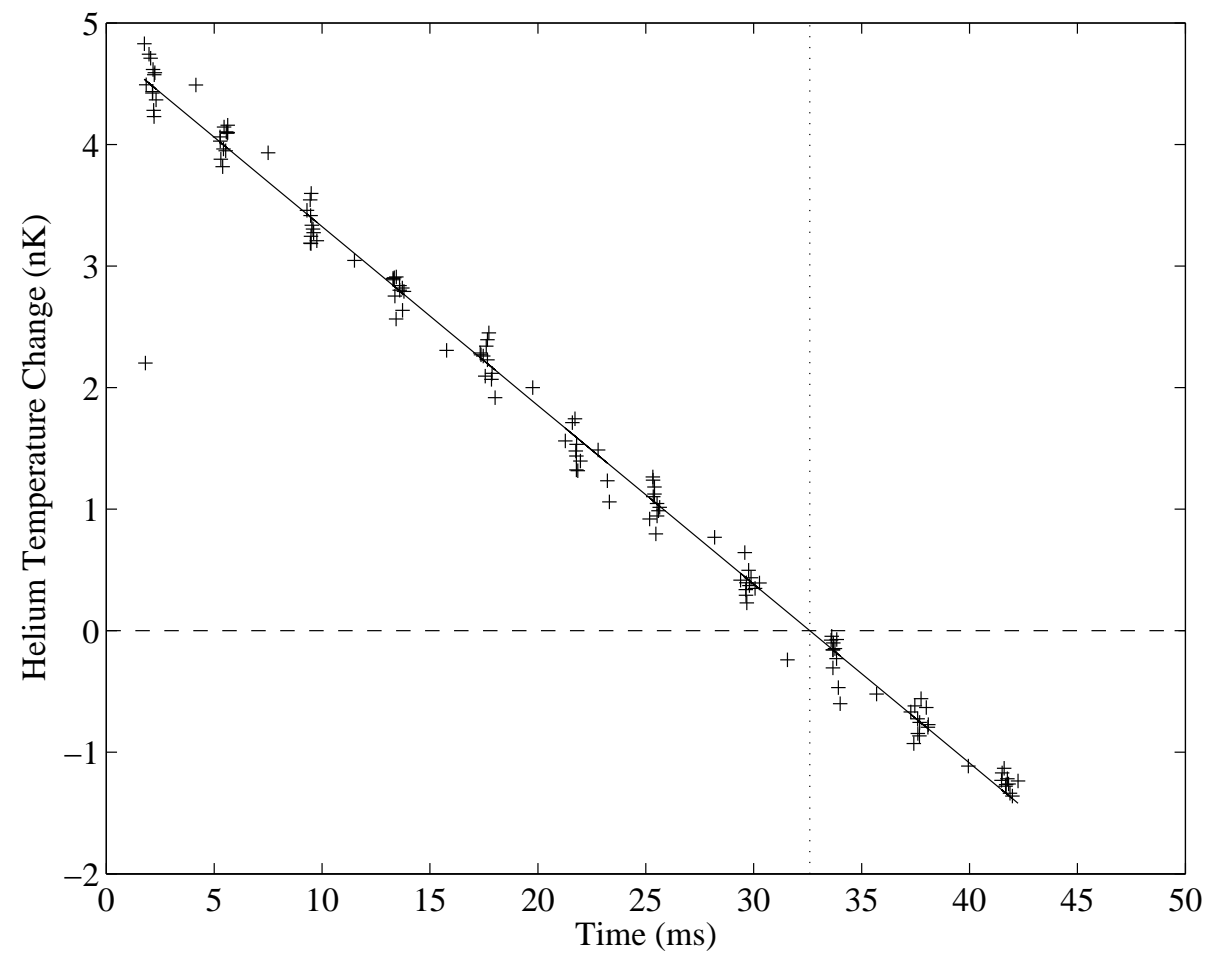

Figure 3.11. Measurement of cell heater response time. The dashed line marks zero temperature change. The dotted line marks the required delay (which in this case is $32.6 \mathrm{~ms}$ ) to get no change in the helium temperature. 


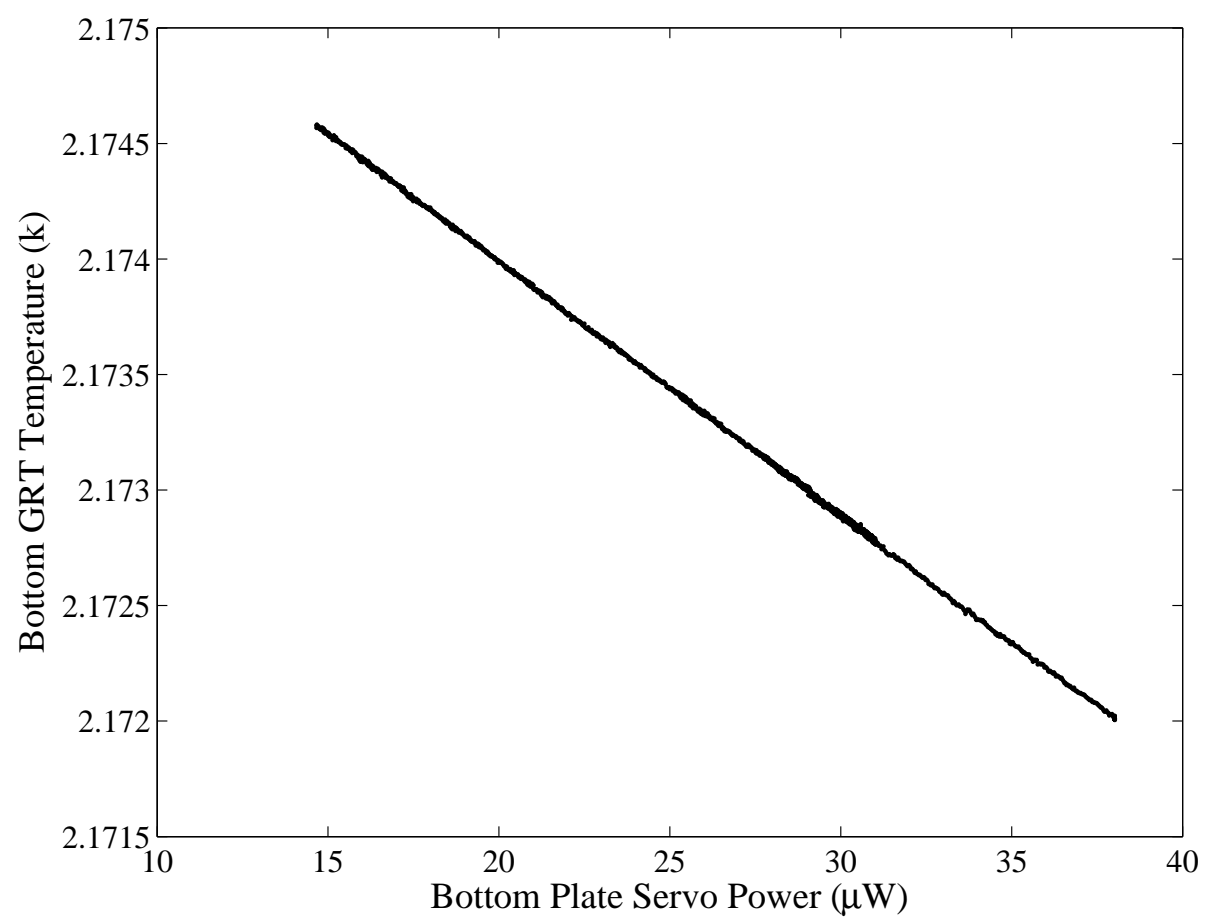

Figure 3.12. Dependence of GRT bottom on heat flux.

\subsubsection{The Problem}

To quantify the effect, we performed a simple experiment. We controlled the cell bottom HRT with a servo to a set flux value and then ramped the stage 3 temperature up and down. Since the heat flux extracted from the cell is determined by the temperature difference between the cell and stage 3 , the servo power went down and up to compensate. In fig. 3.12, we plot the cell bottom GRT versus the servo power. Since the cell bottom GRT is mounted on the heat sinking foil and not on the cell bottom endplate itself, we can see the magnitude of the problem. Despite the cell bottom endplate remaining constant in temperature, the heat-sinking foil is changing in temperature by over $1 \mathrm{mK}$. Therefore the foils which we had hoped would be at the cell bottom temperature are actually offset in temperature due to a resistance of over $100 \mathrm{~K} / \mathrm{W}$.

With just a couple of exceptions, all the experiments we performed in this cooldown kept the temperature difference between the cell and stage 3 nearly constant. When that is the case, the heat leaks will be constant during the experiment. The first exception is HRT calibration, where we were careful and used the top HRT and top GRT, which are immune from this type of heat leak. The second exception is the experiments described in this section.

In a typical configuration, the total power flowing from the cell to stage 3 was $20 \mu \mathrm{W}$. Given an estimated thermal resistance of a Vespel holder of $2 \times 10^{6} \mathrm{~K} / \mathrm{W}$, the heat leak out of each thermometer is about $1 \mathrm{nW}$. 
A heat leak causes two problems. The first is the leak could reduce or distort the heat flux. Our experiments are not particularly sensitive to these effects since our heat fluxes are relatively large. Only at our smallest heat fluxes, $30 \mathrm{nW} / \mathrm{cm}^{2}$, will leaks on the order of $1 \mathrm{nW}$ be noticeable. The second effect is caused by the heat leak flowing across a thermal resistance through the HRT and out the HRT support. This causes the HRT to read a temperature that is offset from the true temperature. As long as the thermal resistance remains the same, however, the offset will be constant. Therefore, the heat leak through $\mathrm{HRT}_{\mathrm{T}}$ and $\mathrm{HRT}_{\mathrm{B}}$ can be ignored. However, the heat leak through the foil HRT flows across a helium/copper boundary which has a Kapitza resistance. The singular component of the Kapitza resistance is strongly dependent on the helium temperature close to $T_{\lambda}$ and therefore $\mathrm{HRT}_{\mathrm{M}}$ has a variable temperature error that prevents it from reading the true helium sample temperature.

\subsubsection{The Solution}

Luckily, we mounted a heater on the copper bar connecting $\mathrm{HRT}_{\mathrm{M}}$ with the penetrating sidewall foil. Therefore, if we could properly compute how large the heat leak was, we could apply exactly the same amount of heat onto this heater such that no heat would flow across the helium/foil boundary and the temperature offset would be constant.

We measured the heat leak by performing the same experiment as above. We used a servo to control the cell bottom HRT to a particular flux. We ramped the stage 3 temperature. Plotting $\mathrm{HRT}_{\mathrm{M}}-\mathrm{HRT}_{\mathrm{T}}$ vs. the servo power, as shown in fig. 3.13, we find the temperature offset as function of servo power.

We varied the heat current on the $\mathrm{HRT}_{\mathrm{M}}$ mount, and measured a resistance of $25.4 \mathrm{~K} / \mathrm{W}$ from the heater to the helium. ${ }^{3}$ We then used these numbers to compute a foil heat factor $\gamma$ of $1.4410^{-4}$ such that

$$
H_{\text {foil }}=\gamma H_{\text {Total }} .
$$

Therefore, a typical total heat of $20 \mu \mathrm{W}$ gives a foil heat of $2.9 \mathrm{nW}$. This is three times the expected heat leak of $1 \mathrm{nW}$. One possible problem with our technique is that we have only measured the thermal resistance from the location of the heater to the superfluid. If the resistance from the heater location to the thermometer itself were significant, then this method would be incorrect. This could happen if the copper conductivity of the link or thermometer tab was lower than expected or if the indium solder joint cracked, resulting in a larger than expected thermal resistance. Either way, as we show in sec. 4.2.6, there is another way of computing this heat leak that is not subject to these uncertainties. That method gives a foil HRT heat leak of $1.65 \mathrm{nW}$, much closer to the estimated $1 \mathrm{nW}$.

\footnotetext{
${ }^{3}$ We are relatively far from $T_{\lambda}$, so this does not include the contribution of the singular Kapitza resistance.
} 


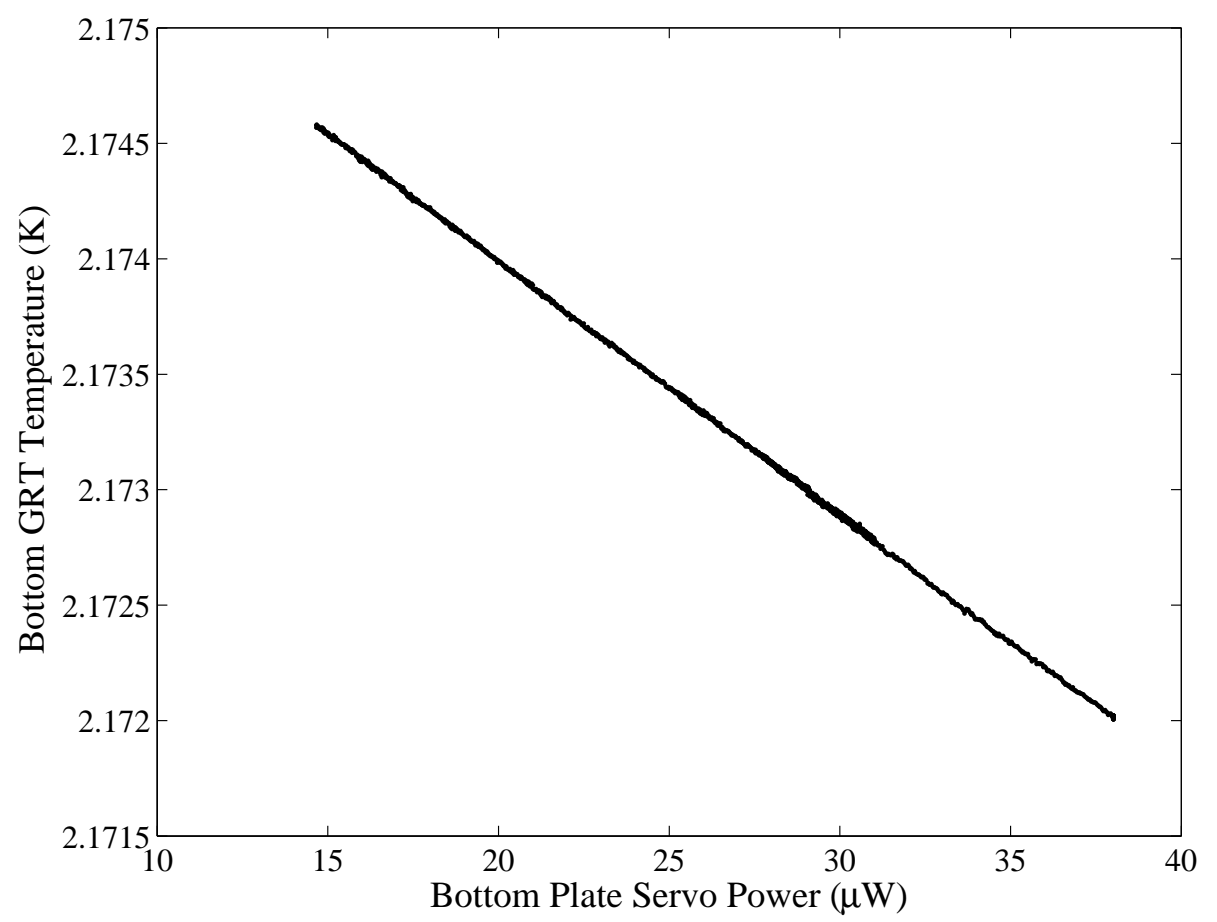

Figure 3.13. Midplane temperature offset vs. servo power needed to maintain temperature difference between cell and stage 3 .

\subsection{Static Heat Capacity}

We performed a traditional pulse heat capacity measurement on our sample of helium in order to determine the amount of helium in our cell and to confirm that our heaters and thermometers were working as expected. We cooled the helium about $10 \mu \mathrm{K}$ below $T_{\lambda}$ at the bottom of our sample. We applied a heat flux of $100 \mathrm{nW} / \mathrm{cm}^{2}$ downwards through the helium because a previous experiment [3] found the following: (1) the heat capacity at this heat flux was unchanged from the static heat capacity; and (2) the arrival of the $100 \mathrm{nW} / \mathrm{cm}^{2}$ SOC state is an excellent measure of $T_{\lambda}$ at the bottom of the cell. ${ }^{4}$ We used the cell bottom heater to minimize any temperature drift of the sample. Then we pulsed upwards, in $1 \mu \mathrm{J}$ increments, until the midplane thermometer saw the SOC state. We ramped down and repeated the measurement four more times.

Our results are plotted in fig. 3.14. With the diameter of the cell from sec. 2.4.2 and the expected amount of helium in the bubble chamber from sec. 3.5, we used the height of the cell as a fitting parameter to match our heat capacity results with a gravity rounded version of the results from the Lambda Point Experiment (LPE) [22]. This gives us the second of three independent measurements

\footnotetext{
${ }^{4}$ Actually, our data analysis places $T_{\mathrm{SOC}}\left(100 \mathrm{nW} / \mathrm{cm}^{2}\right)$ at $11 \mathrm{nK}$ below $T_{\lambda}$.
} 


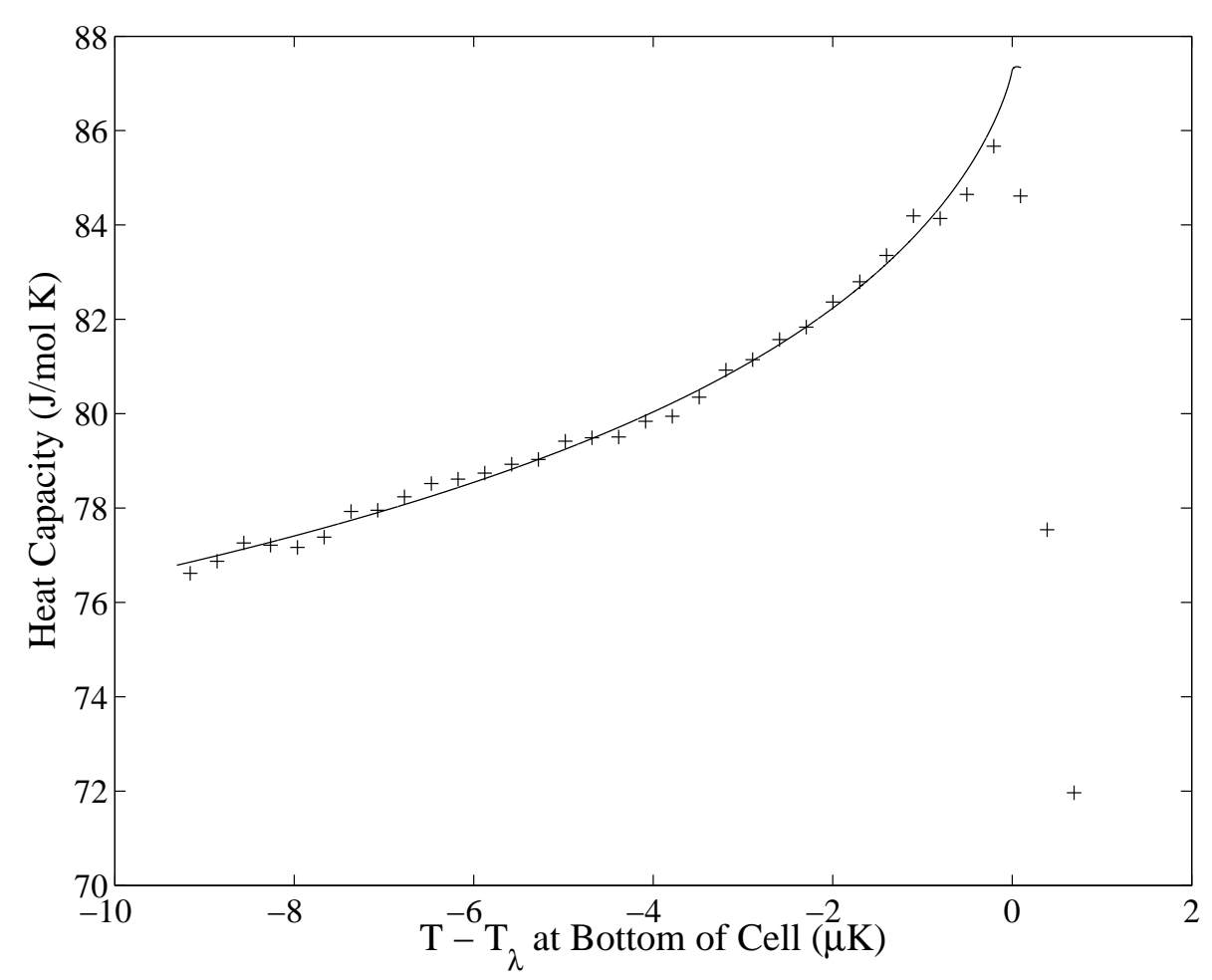

Figure 3.14. Pulse heat capacity results (crosses) compared to the gravity rounded results of LPE (solid line) [22].

of the the number of moles in our sample

$$
n=0.389 \pm 0.001 \mathrm{~mol} \text {. }
$$

There were two odd features of these results. First, the response of the helium to a pulse was not simply a step. Instead, the temperature oscillated about its final temperature for a while with a period of a few seconds and an amplitude of a couple nanokelvin. One such step is shown in fig. 3.15. We guess that the helium in the bubble chamber and the cell itself were out of equilibrium after the sharp change in cell temperature. The capillary linking the two was very fine (0.005" ID) and did not provide a strong enough connection, so a small amount of helium oscillated between the cell and the reservoir as the system came into steady-state.

The second odd feature was the drop in the heat capacity when the temperature at the bottom of the cell is approximately $1 \mu \mathrm{K}$ below $T_{\lambda}$ and the temperature at the fill line is approximately $2.5 \mu \mathrm{K}$ below $T_{\lambda}$ at that point. The drop is consistent with the unlinking of the helium in the bubble chamber $(0.8 \%$ of the total helium) from the measured helium sample. In fig. 3.16 , we plot the results of another heat capacity run with a pulse size of $3.98 \mu \mathrm{J}$. Here the effect is more noticeable because the larger pulse size causes the bubble chamber to thermally disconnect from the main 


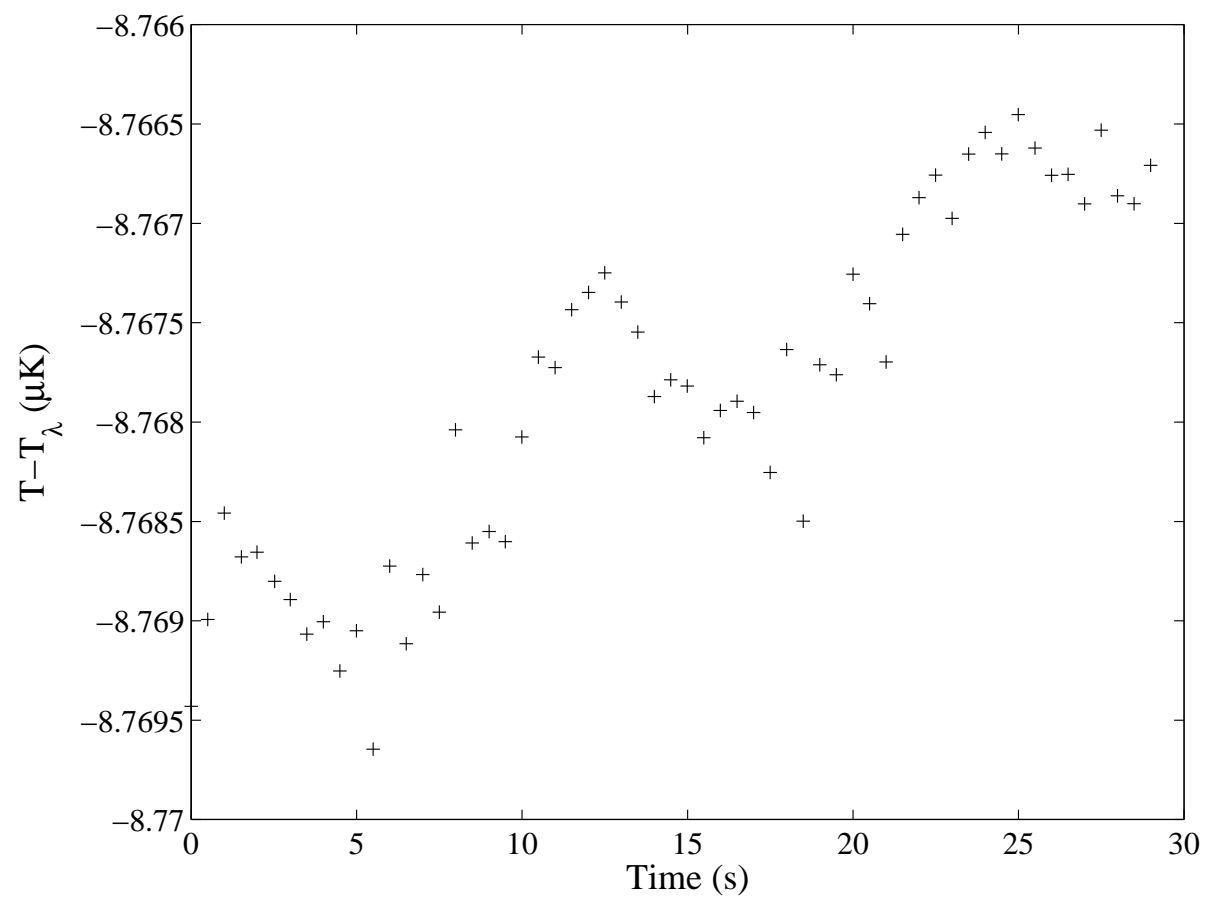

Figure 3.15. The plateau of one temperature step during the pulse heat capacity procedure showing the temperature oscillations seen at each step.

sample at a lower temperature.

\subsection{Emptying the Cell}

At the conclusion of all of our measurements, we performed a simple calibrated extraction to confirm the amount of helium we had in the cell. First, we created the setup shown in fig. 3.17. The calibrated volume is $398.24 \mathrm{~cm}^{3}$. The pressure gauge is a MKS PDR2000 Dual Capacitance Manometer, with a range of 1000 torr. The entire system was isothermal. We filled the calibrated volume with gaseous helium to 865.8 torr and pumped everything else to 0.5 torr. With the valve to the extraction volume closed, we opened the calibrated volume valve. The pressure settled to 751.8 torr. This calibrates the dead volume of the connecting pipework to

$$
V_{\text {dead }}=398.24 \mathrm{~cm}^{3}\left(\frac{865.8-751.8}{751.8-0.5}\right)=60.43 \mathrm{~cm}^{3} .
$$

We increased the pressure in the calibrated volume and dead volume to 953.1 torr. We then opened the valve to the extraction volume. The pressure fell to 52.5 torr. This calibrates our 


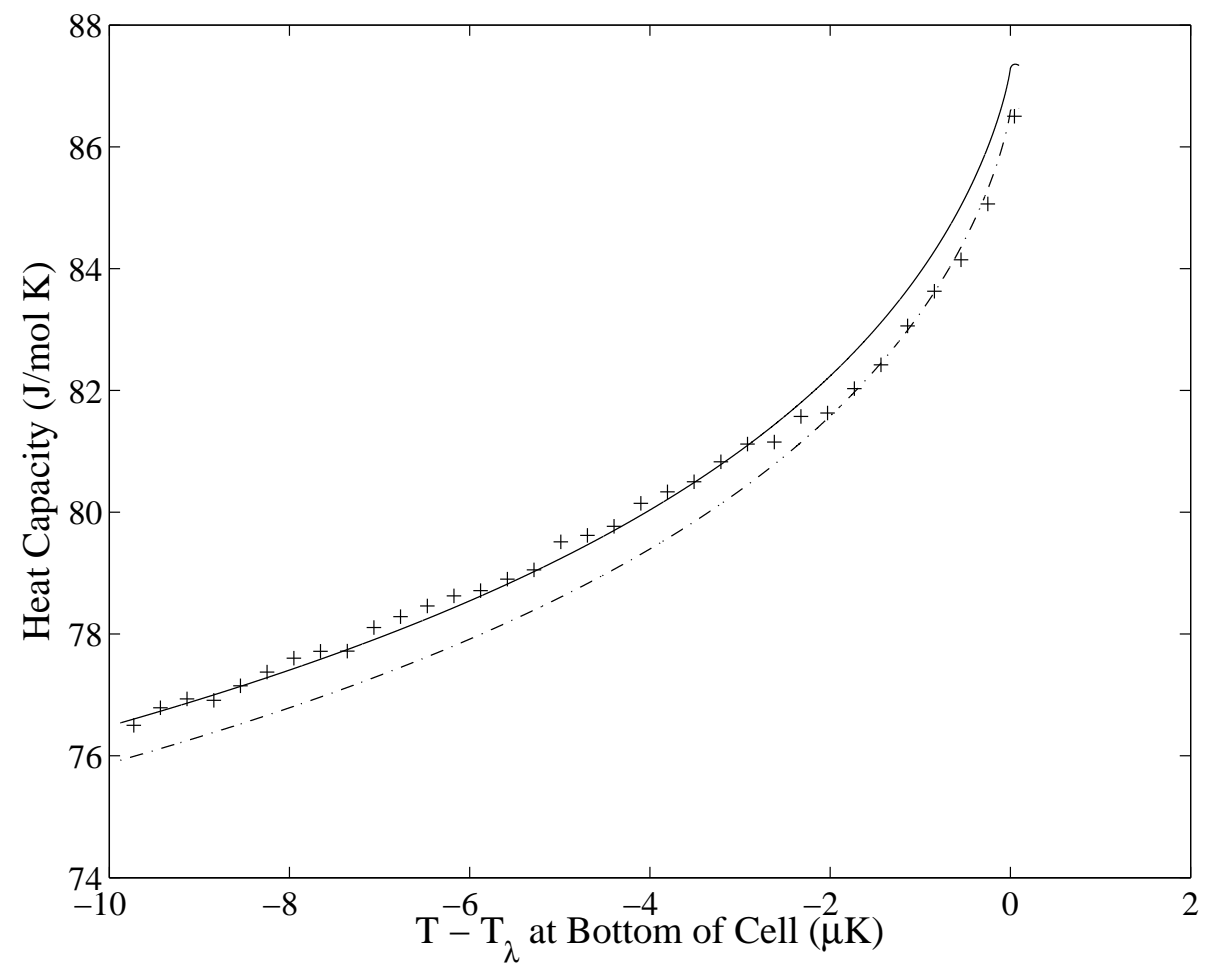

Figure 3.16. Heat capacity results with a $3.98 \mu \mathrm{J}$ pulse size plotted vs. the rounded static heat capacity. The dashed line is the predicted results if the $0.8 \%$ of the helium in the bubble chamber did not contribute to the heat capacity.

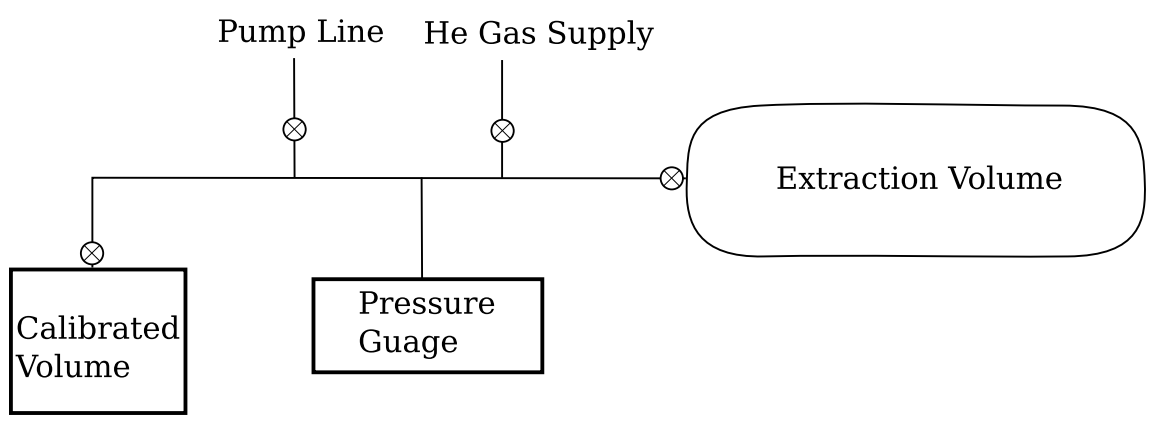

Figure 3.17. Volume calibration schematic. During the extraction, the part of the diagram labeled "calibrated volume" is replaced by the cell fill line. 
extraction volume to

$$
V_{\text {Extract }}=\left(398.24 \mathrm{~cm}^{3}+60.43 \mathrm{~cm}^{3}\right)\left(\frac{953.1-52.5}{52.5-0.5}\right)=7944 \mathrm{~cm}^{3} \text {. }
$$

If we wanted to do a more careful measurement of the extraction, then this measurement would need to be repeated. Calibrating such a large volume from a smaller one leads to significant errors. In this case, assuming all the pressure measurements are \pm 0.1 torr, the error in the extraction volume is $\pm 0.3 \%$.

We then created the same setup except the calibration volume was removed and the cell fill line was connected in its place. Aside from the cell and cell fill line, the system was isothermal. With the cell fill line closed off, we filled the extraction volume to 736.45 torr, pumped out the dead volume to 0.7 torr, and opened $V_{\text {Extract }}$. The pressure fell to 722.6 , which calibrates the dead volume to

$$
V_{\text {dead }}=7944 \mathrm{~cm}^{3}\left(\frac{736.45-722.6}{722.6-0.7}\right)=152.4 \mathrm{~cm}^{3} .
$$

We then pumped $V_{\text {Extract }}$ and $V_{\text {dead }}$ to 0.7 torr and opened the fill line valve at the top of the probe and the cryo-valve by releasing the ${ }^{3} \mathrm{He}$ pressure in the actuator. When the cell was warm, we recorded a pressure of 889 torr at $23{ }^{\circ} \mathrm{C}$. Using drawings we compute the fill line plus cell volume to be $12.3 \mathrm{~cm}^{3}$. Using $P V=n R T$, the number of moles of helium is given by

$$
\begin{aligned}
n & =\frac{(889.0 \text { torr }-0.7 \text { torr })\left(7944 \mathrm{~cm}^{3}+152.4 \mathrm{~cm}^{3}+12.3 \mathrm{~cm}^{3}\right)}{8.3145 \mathrm{~J} / \mathrm{mol} . \mathrm{K} \times 296 \mathrm{~K}} \\
& =0.390 \pm 0.002 \mathrm{~mol} .
\end{aligned}
$$

The error came from the calibrated volume error of $\pm 0.3 \%$ and a temperature error of $\pm 1{ }^{\circ} \mathrm{C}$. We have three independent methods of computing the number moles in the sample: using the cell dimensions and the molar volume of helium, measuring the heat capacity of the sample, and performing the calibrated extraction. Thankfully, they all agree, as shown in table 3.7.

Table 3.7. Moles in helium sample

Volume Calculation $\quad 0.390 \pm 0.002 \mathrm{~mol}$

Heat Capacity $\quad 0.389 \pm 0.001 \mathrm{~mol}$

Calibrated Extraction $\quad 0.390 \pm 0.002 \mathrm{~mol}$

\subsection{Summary}

We have described the apparatus and calibration procedures. We are now ready to discuss our two main experiments: measuring $C_{\nabla T}$ and measuring the speed and attenuation of the SOC wave. 
There is still one open question, however. As of now, we do not have a satisfactory measurement of the heat leak through the midplane foil, which may affect our measurements. This will be resolved in the next chapter. 


\section{Chapter 4}

\section{SOC Heat Capacity}

The SOC state of helium exists both above and below the superfluid transition temperature, $T_{\lambda}$. As discussed in sec. 1.1, the presence of the phase transition has not yet been seen in the properties of the SOC state. This has led to speculation that the transition temperature is depressed to $T_{\lambda}(Q)$ and that the SOC state always exists on the normal side of the depressed superfluid transition. If we could measure the heat capacity of the SOC state, we could gain insight into how the heat flux and temperature gradient affect the phase transition.

\subsection{Procedure}

A traditional heat capacity measurement involves putting a bit of energy into a system and measuring the resulting temperature change. However, a pulse of energy does not change the temperature of the SOC state. The only way to change the temperature of the SOC state is to change the heat flux. This requires developing a new procedure, but the basics still remain the same: the amount of energy required to create a known temperature change must be accurately measured.

\subsubsection{Procedure Overview}

Fig. 4.1 shows temperature profiles of the helium sample during various parts of the experimental procedure. In addition, fig. 4.2 shows data corresponding to one such measurement. We will walk through the procedure relating each step with the corresponding profile in the schematic and time in the experimental data. 


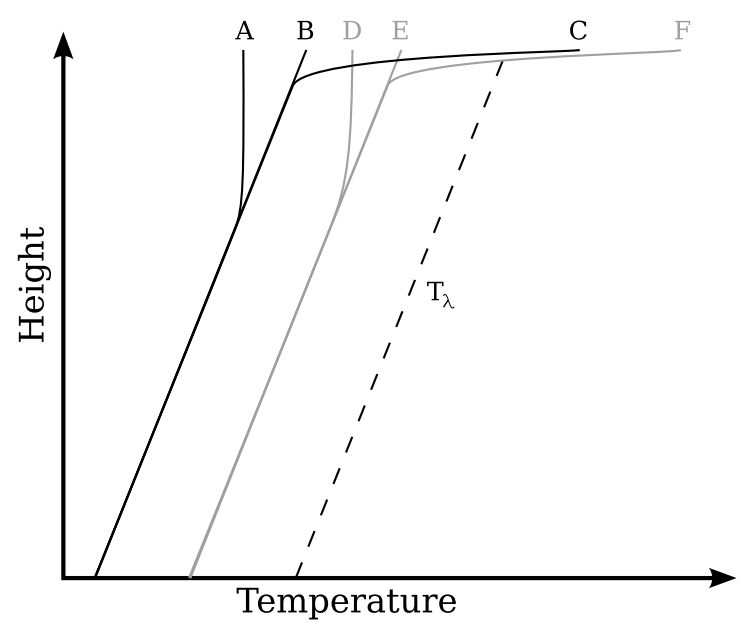

Figure 4.1. Schematic of the temperature profiles during the SOC state heat capacity measurement. The SOC temperatures are drawn colder than $T_{\lambda}$. For low heat fluxes, the SOC temperature is above $T_{\lambda}$. The meaning of the labels is explained in the text.
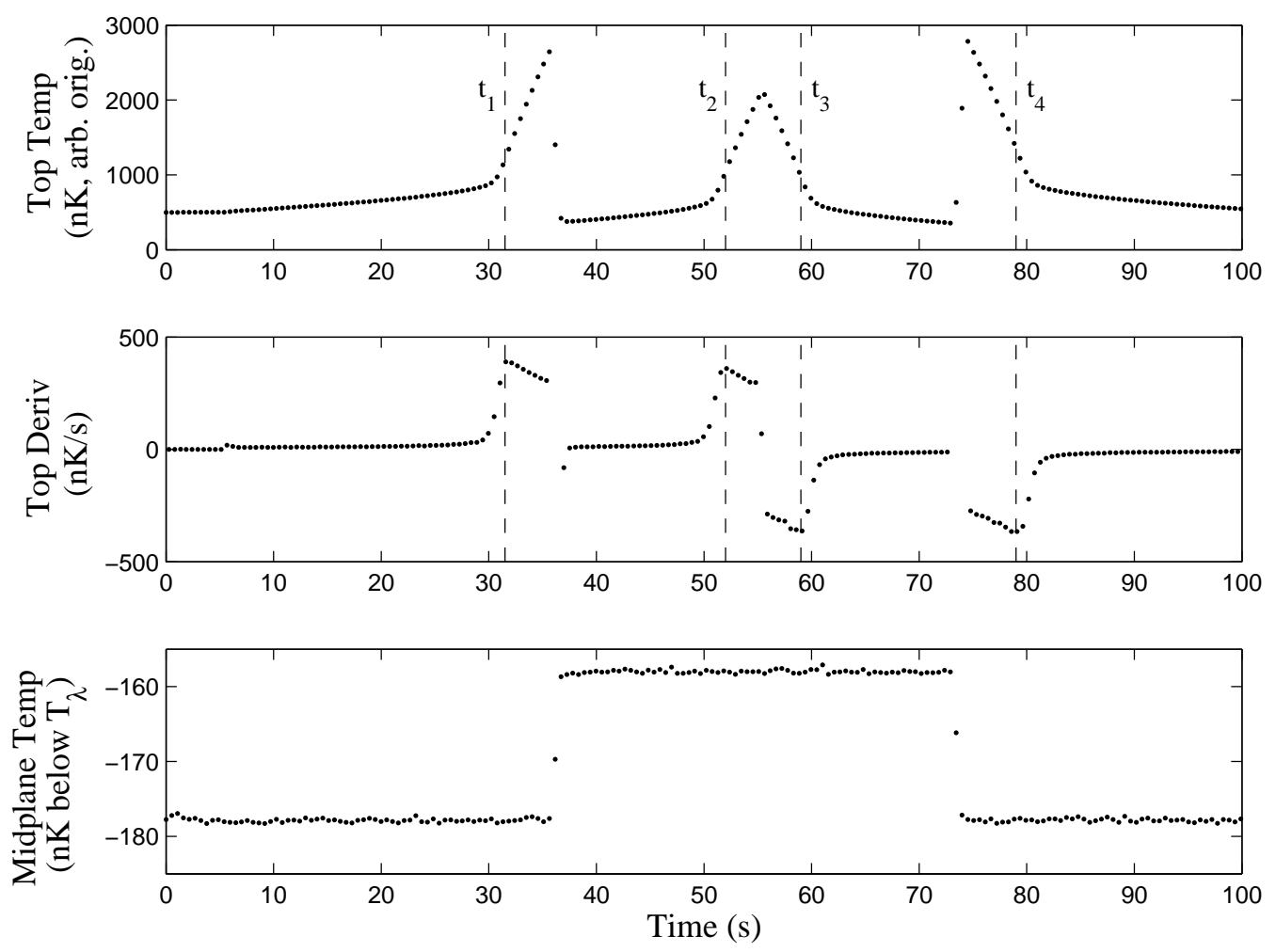

Figure 4.2. Data time series for one SOC heat capacity data point. The initial heat flux is $Q_{1}=$ $1.47 \mu \mathrm{W} / \mathrm{cm}^{2}$ and the second is $Q_{2}=1.27 \mu \mathrm{W} / \mathrm{cm}^{2}$. 
Initially, at $t=0 \mathrm{~s}$, the helium is mostly in the SOC state with some superfluid at the top of the sample, as in profile A. A heat flux $Q_{1}$ is flowing through the SOC state, and approximately $Q_{1} \times A$, where $A$ is the cell endplate area, is being added with the top heaters. At $t=6 \mathrm{~s}$, the top heater power is increased by a ramping heat $\delta H$, and the top temperature begins to drift up.

The helium in the SOC state cannot change temperature, so as energy is added the superfluid layer above the SOC state warms and the interface between the SOC state and the superfluid moves up the sample. As the depth of the layer that changes temperature gets thinner and thinner, the heat capacity is reduced and the top temperature derivative increases. The derivative continues to increase until, at $t_{1}=32 \mathrm{~s}$, it reaches a maximum. This corresponds to profile B. At this point, normal fluid penetrates the top of the sample and begins to travel down the sample. This continues and profile $\mathbf{C}$ is reached.

At $t=36 \mathrm{~s}$, the bottom heater power is increased by $\Delta Q \times A$ and the top heater power is decreased by $\Delta Q \times A$. Now, $Q_{2}=Q_{1}-\Delta Q$ is flowing through the SOC state, and $Q_{2} \times A+\delta H$ is being added to the top heaters. The helium quickly $(\sim 1 \mathrm{~s})$ relaxes to profile D. ${ }^{1}$ Since an excess of heat is still being added, the cell top temperature continues to warm.

The derivative maximum at $t_{2}=52 \mathrm{~s}$ corresponds to profile E. Normal fluid again penetrates and we reach profile $\mathbf{F}$ at $t=55 \mathrm{~s}$. Then the top heat is reduced to $Q_{2} \times A-\delta H$ and the entire process is reversed: profile $\mathbf{E}$ at $t_{3}=59 \mathrm{~s}$, profile $\mathbf{D}$ at $73 \mathrm{~s}$ where the heat flux is changed back to $Q_{1}$ and the top heat is switched to $Q_{1} \times A-\delta H$, profile $\mathbf{C}$ at $t=75 \mathrm{~s}$, profile $\mathbf{B}$ at $t_{4}=79 \mathrm{~s}$ and profile $\mathbf{A}$ at $t=100 \mathrm{~s}$.

We now have the information necessary to make a first attempt at calculating the heat capacity for this data run. Using the midplane temperature, we can obtain $\Delta T_{\mathrm{SOC}}$. The energy required to go from $T_{\mathrm{SOC}}\left(Q_{1}\right)$ to $T_{\mathrm{SOC}}\left(Q_{2}\right)$ is $\delta H\left(t_{2}-t_{1}\right)$, and the energy to return to $T_{\mathrm{SOC}}\left(Q_{1}\right)$ is $-\delta H\left(t_{4}-t_{3}\right)$. Therefore, the heat capacity is given by

$$
C_{\nabla T}=\frac{\delta H\left(\left(t_{2}-t_{1}\right)+\left(t_{4}-t_{3}\right)\right)}{2 \Delta T_{\mathrm{SOC}}} .
$$

\subsubsection{Maintaining the Balance}

This procedure works best when the heat flux added to the top heaters exactly matches the amount of heat being extracted through the SOC state (aside from the $\delta H$ being added or subtracted). In order to maintain this balance, we could periodically stop the experiment in profile $\mathbf{A}$, and find the heat applied to the top endplate such that the cell top temperature remains constant.

A more expedient method is to use the data from the run itself. During each run, the time $t_{\alpha}$ and top temperature $T_{\alpha}$ is recorded shortly after profile $\mathbf{D}$ is reached for the first time. When profile

\footnotetext{
${ }^{1}$ For for very low $Q$, the relaxation is slower. In chap. 5, we measure the speed of the SOC wave, which governs the speed at which this relaxation occurs.
} 
$\mathbf{F}$ is reached and the top heat is reduced, the time $t_{\beta}$ is recorded. Then, when $T_{\alpha}$ is reached again, ${ }^{2}$ the time $t_{\gamma}$ is recorded. If the heat flux is in balance, $t_{\gamma}-t_{\beta}=t_{\beta}-t_{\alpha}$. If it is not, an adjustment is made to the top heater of

$$
\Delta H_{\text {Top }}=\delta H\left(t_{\gamma}-2 t_{\beta}+t_{\alpha}\right) /\left(t_{\gamma}-t_{\alpha}\right)
$$

\subsection{Analysis}

The procedure described in sec. 4.1 was repeated many times for different heat fluxes. Data were recorded twice a second for each of the HRTs and for the three Fluke multimeters that measure the current through the heaters. We need to extract the temperature change of the midplane thermometer, the absolute temperature of the SOC state (relative to $T_{\lambda}$ ), the ramping heat $\delta H$, and the time between the establishment of one SOC state and the establishment of the other. We will continue to refer to the profiles in fig. 4.1 and the data in fig. 4.2.

\subsubsection{SOC Temperature Change}

The change in the SOC temperature is easy to extract. We average all the points from $\mathrm{HRT}_{\mathrm{M}}$ for each SOC state temperature and take the difference. Since we have previously calibrated $\mathrm{HRT}_{\mathrm{M}}$, the temperature change is apparent. For example, in fig. 4.2, data from $t=6 \mathrm{~s}$ to $t=36 \mathrm{~s}$ are averaged with data from $t=73 \mathrm{~s}$ to $t=100 \mathrm{~s}$ and subtracted from the average of $t=37 \mathrm{~s}$ to $t=72 \mathrm{~s}$. For this set of data, the answer is $\Delta T=19.83 \mathrm{nK}$. From the scatter of identical measurements, the statistical error for $\Delta T$ for the smallest temperature step size of $\approx 20 \mathrm{nK}$ was $0.5 \%$.

There are only two complications. First, on occasion, there was a spike in the value of the HRT. This was caused by a sudden increase in vibrational heating (e.g., a slammed door or something in the lab dropped or moved). The value quickly relaxed to nominal in a couple of seconds. We remove these spikes by customizing our function to perform the average. We sort the values from lowest to highest and drop the bottom and top quarters. We then do a simple mean on the remaining half. This almost completely removes the effect of the spike with only a minuscule remaining bias. ${ }^{3}$

Second, and much more importantly, the heat leak discussed in sec. 3.10 can produce a changing temperature offset for low heat fluxes. In the data shown in fig. 4.2, there is not much of an effect because, between $160 \mathrm{nK}$ and $180 \mathrm{nK}$ below $T_{\lambda}$, the singular component of the Kapitza resistance does not change significantly. At low heat fluxes, however, the SOC temperature is very close (or

\footnotetext{
${ }^{2}$ To be precise, we wait for $T_{\alpha}-2 \delta H R_{\mathrm{K}, \mathrm{T}}$, where $R_{\mathrm{K}, \mathrm{T}}$ is the top endplate Kapitza resistance.

${ }^{3}$ Ignoring the effect of the spike, the temperature data that would have been measured are equally likely to be high and low. Therefore, throwing these points out as the "high" points does not completely balance the "low" points we have thrown out. If the spike was $2 \mathrm{~s}$ out of 30 , then the bias is approximately $2 \sigma / 30=.03 \mathrm{nK}$, which is below our HRT noise level.
} 
sometimes above) $T_{\lambda}$ and the effect can be quite large. We will discuss in sec. 4.2.6 how we fixed this potential error and why the correction discussed in sec. 3.10 did not work.

\subsubsection{Ramping Heat $\delta H$}

The ramping heat is computed using the voltages across the precision resistors measured by the Fluke multimeter and the heater calibrations discussed in sec. 3.2. Half the difference between the total applied heat on the first ramp up and on the last ramp down is one determination of the ramping heat. Another determination comes from half the difference of the total heat applied on the middle ramps. We use the average of these two in our calculations. In the case of the data in fig. $4.2, \delta H=35.10 \mathrm{nW}$. The error in the ramping heat for all data runs was always less than $0.3 \%$.

\subsubsection{Time Interval}

We need to extract the time interval from when one SOC state fills the sample, profile $\mathbf{B}$, until the other SOC state fills the sample, profile E. Initially, we used the maximum derivative of $\mathrm{HRT}_{\mathrm{T}}$ as the marker of the fully established SOC state. However, noise on the HRT as well as the $0.5 \mathrm{~s}$ time resolution lead to poor localization of the maximum. The error was typically 5 to 10 percent and resulted in a large scatter in the heat capacity results. We can improve the results by dealing with the full derivative curve.

The algorithm we use goes as follows. All the calculations use the derivatives of $\mathrm{HRT}_{\mathrm{T}}$. We divide the output into four curves $\left(C_{1}\right.$ to $\left.C_{4}\right)$, one for each of the ramps through a full SOC state. From fig. $4.2, t=6 \mathrm{~s}$ to $36 \mathrm{~s}$ is $\mathrm{C}_{1}(t), t=37 \mathrm{~s}$ to $55 \mathrm{~s}$ is $\mathrm{C}_{2}(t), t=56 \mathrm{~s}$ to $72 \mathrm{~s}$ is $\mathrm{C}_{3}(t)$, and $t=74 \mathrm{~s}$ to $100 \mathrm{~s}$ is $\mathrm{C}_{4}(t)$. We use the time difference between the half-maximuma of $\mathrm{C}_{1}$ and $\mathrm{C}_{2}$ to get an estimate for the time interval $\tau_{1}$.

We then improve the estimate by minimizing

$$
\sum\left|\mathrm{C}_{1}(t)-\mathrm{C}_{2}\left(t-\tau_{1}\right)\right| / L
$$

by adjusting $\tau_{1}$ up and down by units of $0.05 \mathrm{~s}$ to find a local minimum. In order to avoid the heat flux dependent part of the curve, the difference is only done over the overlap of the two curves up to $70 \%$ of the maximum derivative of the shifted curve. $L$ is proportional to the length of region differenced. The first curve is linearly interpolated to allow shifts of less than $0.5 \mathrm{~s} . \tau_{2}$ is found in the same manner using curves $\mathrm{C}_{3}$ and $\mathrm{C}_{4}$. For the data set shown in fig. 4.2 , the values are $\tau_{1}=20.28 \mathrm{~s}$ and $\tau_{2}=20.32 \mathrm{~s}$.

Fig. 4.3 shows the $\mathrm{HRT}_{\mathrm{T}}$ derivative curves for data sets at different heat fluxes. As can be seen in Figs. 4.3(a), 4.3(b), and 4.3(c), our algorithm does an excellent job of finding the time difference between the two curves. However, as seen in fig. $4.3(\mathrm{~d})$, there is some ambiguity at very low heat 
fluxes. The derivative maximuma are not coincident when the curves are matched. Here, the small change in heat flux we use to make our measurement is a large percentage of the total heat flux. Also, the measurement is occurring very close to $T_{\lambda}$ where there are large changes in singular Kapitza resistance and there is a long healing length. ${ }^{4}$ Under these conditions our algorithm does not find the correct difference in the derivative maximuma. However, there is no way to know if the correct signature of the fully formed SOC state at these low heat fluxes is a maximum in the derivative of $\mathrm{HRT}_{\mathrm{T}}$, nor if there ever truly is a "fully formed SOC state." We made a numerical calculation modeling the SOC state as a normal fluid with the thermal conductivity given by eq. (1.16). The calculation suggests that the maximum in the derivative does not necessarily occur at the fully formed SOC state for very low heat fluxes. ${ }^{5}$ Therefore, we have stuck with our algorithm which gives consistent and low noise results. The statistical error (derived from the scatter of identical measurements) is $<1.5 \%$ for this method and $5 \%$ to $10 \%$ using the derivative maxima. However, it is entirely possible that there is a systematic error at low heat fluxes. Using the difference between our measurement of $\tau_{1}$ and $\tau_{2}$ and the time difference between the derivative maxima as a guide, we estimate that this error is less than $0.5 \%$ for $T_{\mathrm{SOC}}<50 \mathrm{nK}$ below $T_{\lambda}$, less than $1 \%$ for $50 \mathrm{nK}<T_{\mathrm{SOC}}<30 \mathrm{nK}$ and less than $2 \%$ for the remainder of our results.

\subsubsection{The Uncorrected Heat Capacity}

We have all the pieces now. The heat capacity is given by

$$
C=\frac{\delta H\left(\tau_{1}+\tau_{2}\right) / 2}{\Delta T}
$$

For example, given our values we extracted from the data set shown in fig. 4.2 and knowing that we have 0.386 moles of helium, ${ }^{6}$ we can compute a molar heat capacity of

$$
\begin{aligned}
C & =\frac{35.10 \mathrm{nW}(20.28 \mathrm{~s}+20.32 \mathrm{~s}) / 2}{19.83 \mathrm{nK} 0.386 \mathrm{~mol}} \\
& =93.1 \mathrm{~J} / \mathrm{mol} . \mathrm{K}
\end{aligned}
$$

The most significant statistical error is from the measurement of the time shifts, $\tau_{1}$ and $\tau_{2}$. This gives approximately a $1 \%$ statistical error in our heat capacity measurements.

\footnotetext{
${ }^{4}$ The healing length is the intermediate region between the superfluid and the SOC state over which the gradient transitions between no thermal gradient and a gradient of $\nabla T_{\lambda}$.

${ }^{5}$ This model did not include the singular Kapitza resistance and the heat capacity of the top endplate. In addition, the model does not accurately predict the temperature profile in the crossover region between superfluid and SOC state, so the calculation, while instructive, is not suitable for numerical predictions.

${ }^{6}$ In sec. 3.11, we made the determination that the bubble chamber is not thermally connected to the main chamber of helium. Therefore, the number of moles in the sample is reduced by $0.8 \%$ from 0.389 moles.
} 


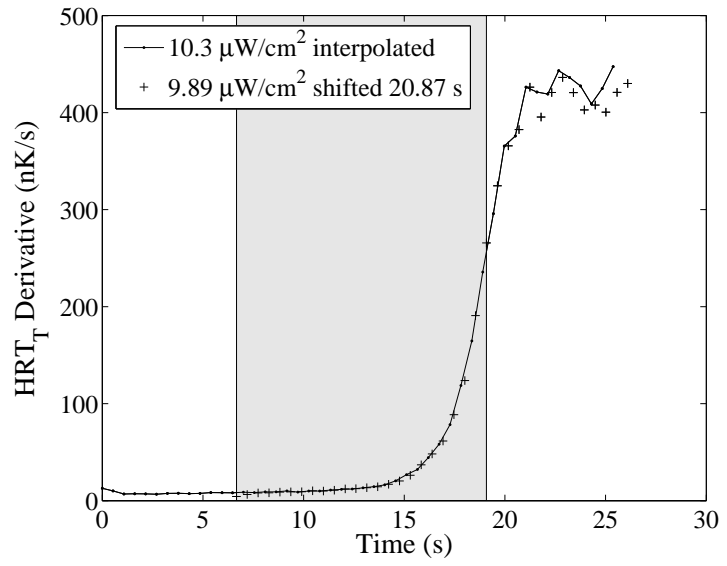

(a) $T_{\mathrm{SOC} 1}=899 \mathrm{nK}$ and $T_{\mathrm{SOC} 2}=870 \mathrm{nK}$ below $T_{\lambda}$. At high heat flux the derivatives of the two curves match excellently, although the derivative is noisy when normal fluid has entered the cell at $t>21 \mathrm{~s}$.

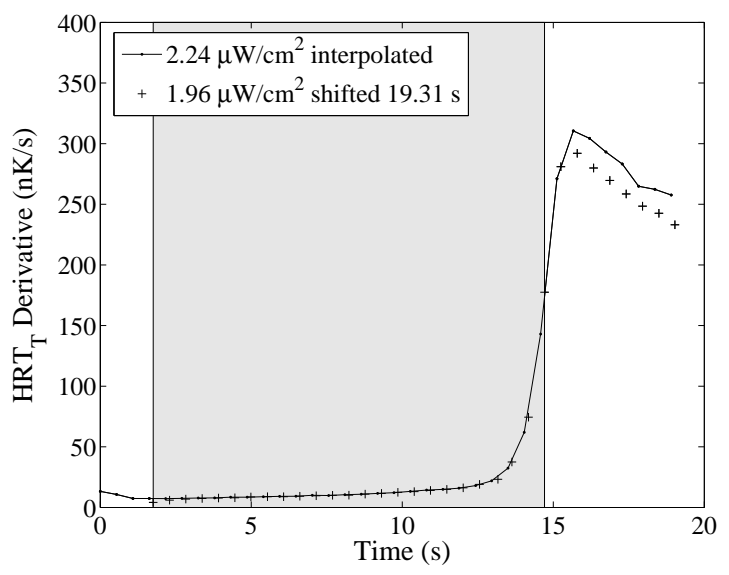

(c) $T_{\mathrm{SOC} 1}=252 \mathrm{nK}$ and $T_{\mathrm{SOC} 2}=227 \mathrm{nK}$ below $T_{\lambda}$. At moderately low heat flux the derivatives match excellently over the shaded region, with a noticeable difference when normal fluid enters the cell at $t>15 \mathrm{~s}$. However, there is no significant time difference between the derivative maxima.

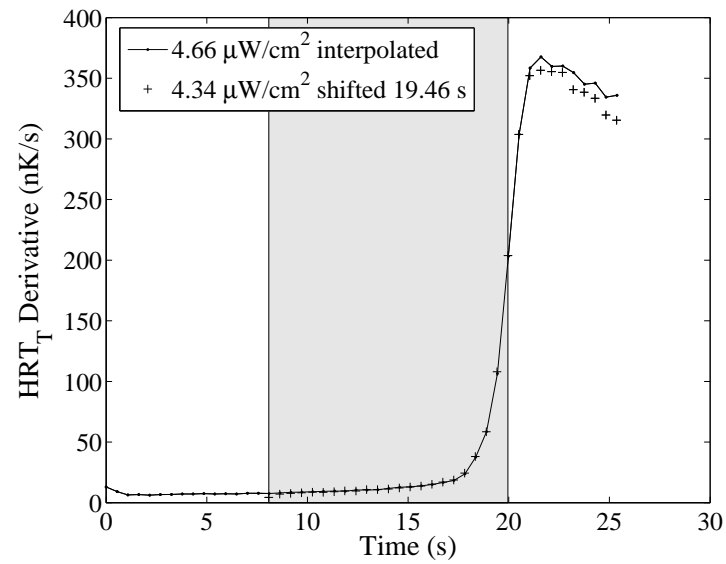

(b) $T_{\mathrm{SOC} 1}=458 \mathrm{nK}$ and $T_{\mathrm{SOC} 2}=432 \mathrm{nK}$ below $T_{\lambda}$. At moderate heat flux, the derivatives match excellently, with a slight difference when normal fluid enters the cell at $t>21 \mathrm{~s}$.

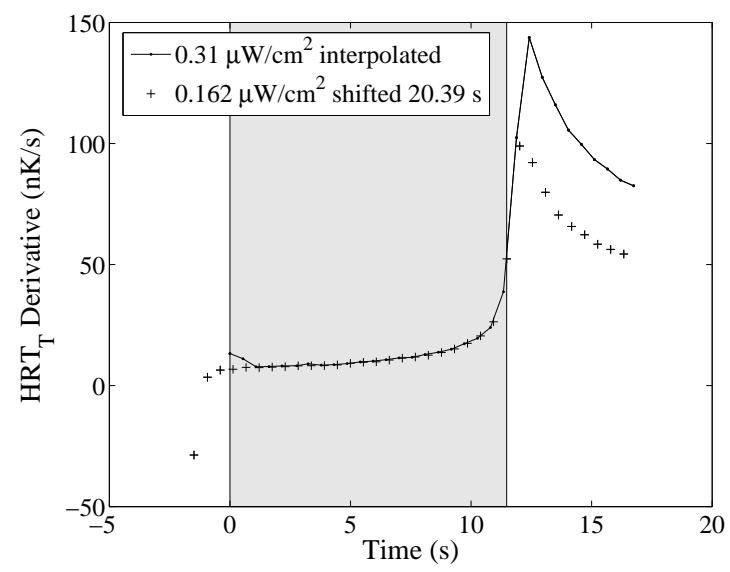

(d) $T_{\mathrm{SOC} 1}=51 \mathrm{nK}$ and $T_{\mathrm{SOC} 2}=27 \mathrm{nK}$ below $T_{\lambda}$. At low heat flux the derivatives match excellently of the shaded region, but there is a significant difference when the normal fluid enters the cell at $t>12 \mathrm{~s}$. There is also a significant $(\approx 0.4 \mathrm{~s}$ or $2 \%$ ) difference in the maximum derivative.

Figure 4.3. Examples of matching the $\mathrm{HRT}_{\mathrm{T}}$ derivative curves. Eq. (4.3) is minimized over the shaded region. 


\subsubsection{Heater Response Time Correction}

There is still some work to do in order to accurately determine the heat capacity from our measurement. As heat is swapped from one heater to the other, a small amount of energy is lost. After each heat capacity run (a run usually took less than 24 hours), the correction procedure described in sec. 3.9 was performed. The correction was on the order of a few percent $(<2 \%$ at the low heat fluxes and $\approx 6 \%$ at the highest heat fluxes). In the case of the data run in fig. 4.2, $21 \mathrm{~nJ}$ was lost during the switch, so

$$
\begin{aligned}
C & =\frac{35.10 \mathrm{nW}(20.52 \mathrm{~s}+19.98 \mathrm{~s}) / 2+21 \mathrm{~nJ}}{19.83 \mathrm{nK} 0.386 \mathrm{~mol}} \\
& =95.9 \mathrm{~J} / \mathrm{mol} . \mathrm{K}
\end{aligned}
$$

We estimate that the correction is computed to better than $5 \%$. This leads to a systematic error of less than $0.3 \%$, which is small enough to be lost in our statistical noise.

\subsubsection{Foil Heat Leak}

We mentioned in sec. 3.10 that there is reason to be concerned about whether we computed the heat leak through the midplane foil correctly. If we are incorrect, the measurement of the SOC temperature will be off, especially for low heat fluxes where the helium self-organizes near $T_{\lambda}$. As shown in fig. 4.4, the measurement of the SOC heat capacity is especially susceptible to the effects of the heat leak. We varied the heat applied to the foil (and $\gamma$ by $H_{\text {foil }}=\gamma H_{\text {Total }}$ ) and either an enhancement or a depression of the heat capacity can be seen. The foil heat calculated in sec. 3.10 has $\gamma=1.4410^{-4}$ and gives a very large heat capacity depression at low heat fluxes.

In order to find out what the correct $\gamma$ is, we can take data sets at different stage 3 temperatures which leads to different total heats extracted from the experiment stage. If the heat leak is correctly compensated for with a choice of $\gamma$, then the results will be independent of the total heat extracted from the cell. In fig. 4.5, heat capacity results for one choice of $\gamma$ and different total heats are plotted on the same graph. Since the curves match, this gives us the $\gamma$ we used for all the remaining data sets, $\gamma=8.2610^{-5}$.

One complication that was neglected in the above discussion is a source of noise we found on our foil heater. We used the Keithley 263 Calibrator to apply the electrical current to the foil heater. When the cable from the Keithley was plugged into the foil heater connector on the breakout box, there was a noticeable increase in the foil temperature. Apparently, either the cable was acting as an antenna and depositing heat or the Keithley output some current even when set to zero. We used the measured Kapitza resistance of the midplane foil to calculate $0.3 \mathrm{nW}$ of heating. The $0.3 \mathrm{nW}$ was taken into account for both these calibration measurements and the final measurements reported 


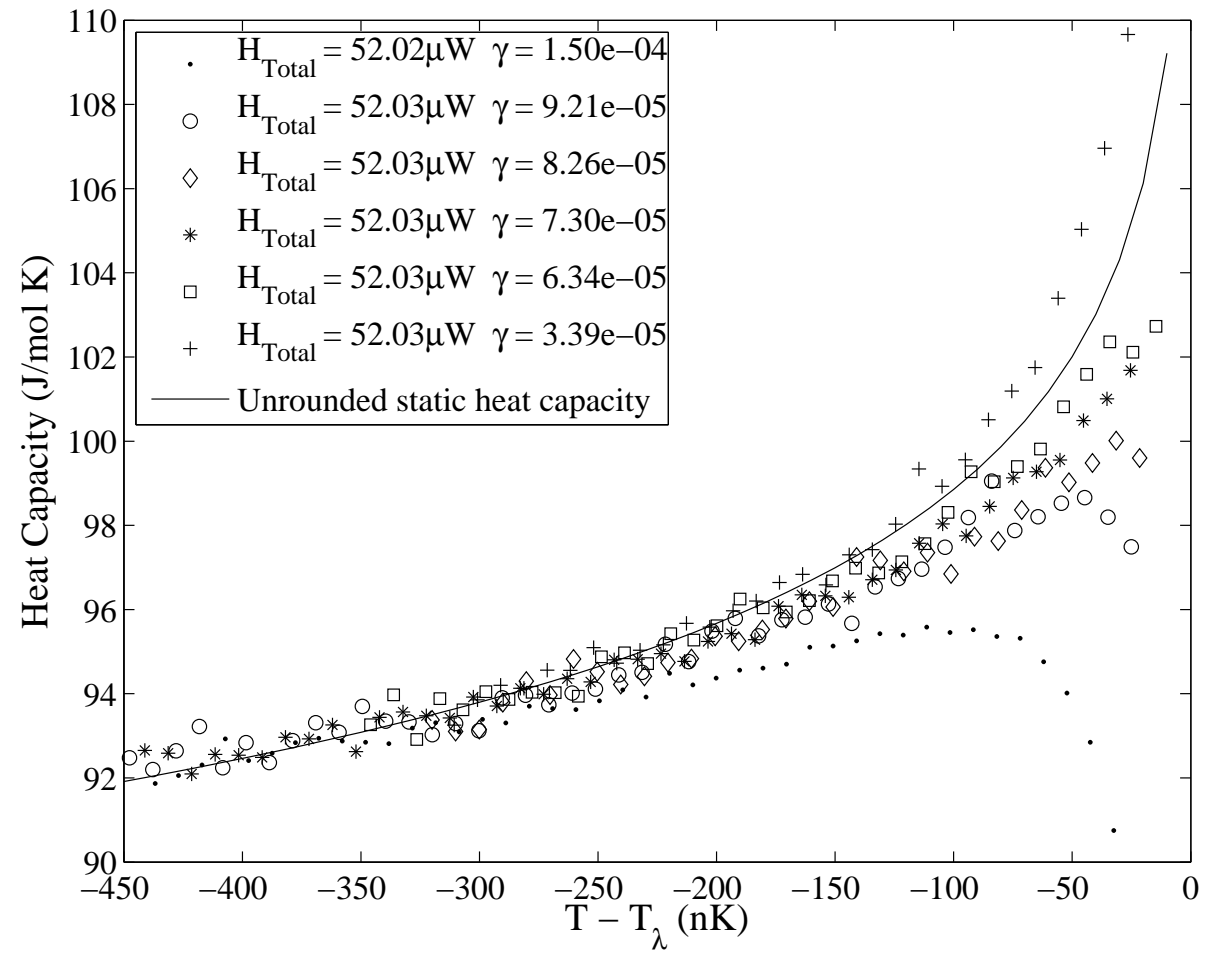

Figure 4.4. SOC heat capacity results for different foil heats. The legend lists the total heat passing from the cell stage to stage 3 as well as the foil heat factor $\gamma$ from eq. (3.4). The actual foil heat applied ranges from 1.8 to $7.8 \mathrm{nW}$. 


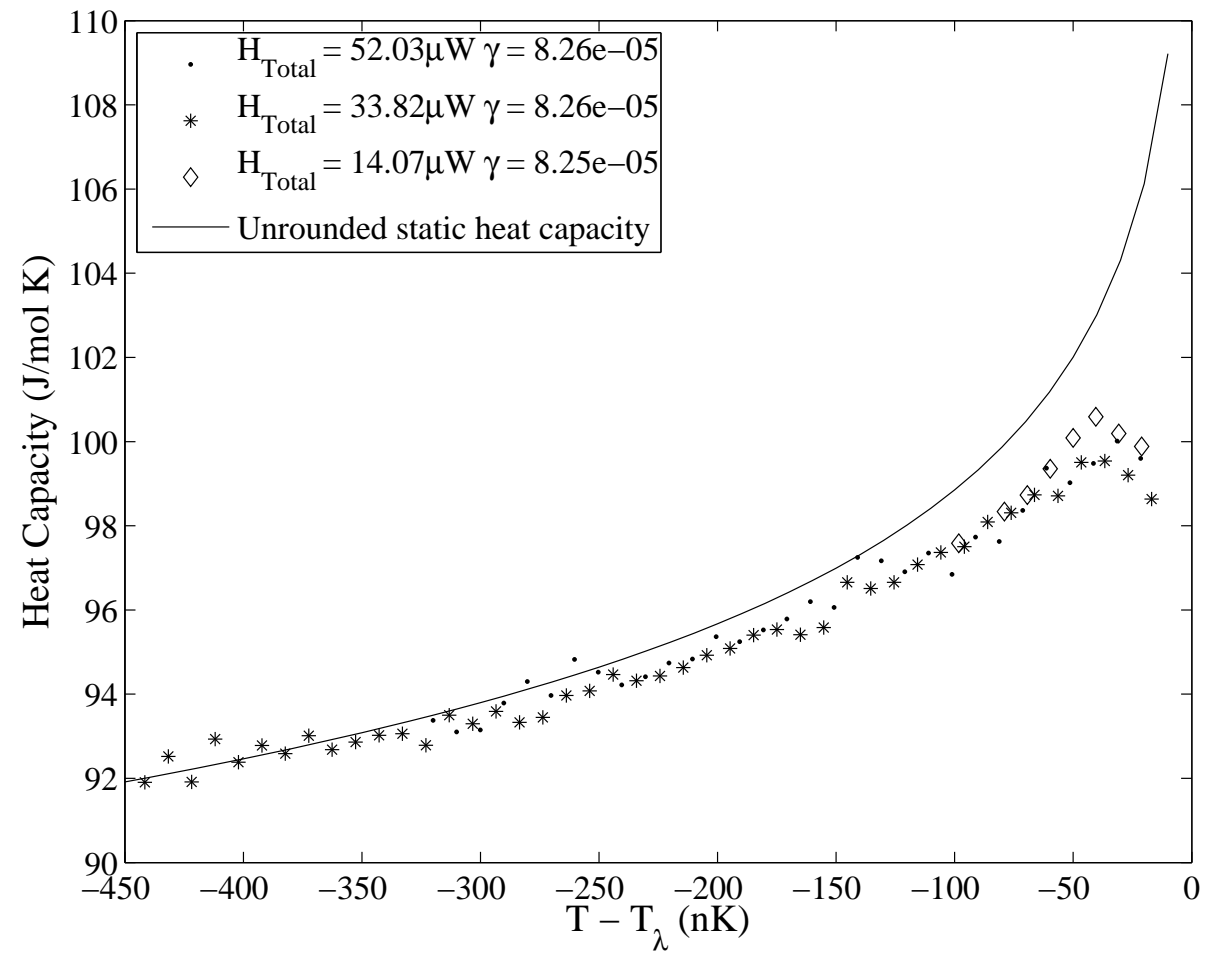

Figure 4.5. SOC heat capacity results to determine the foil heat factor $\gamma$. Different total heats using the same $\gamma$ give the same results. 


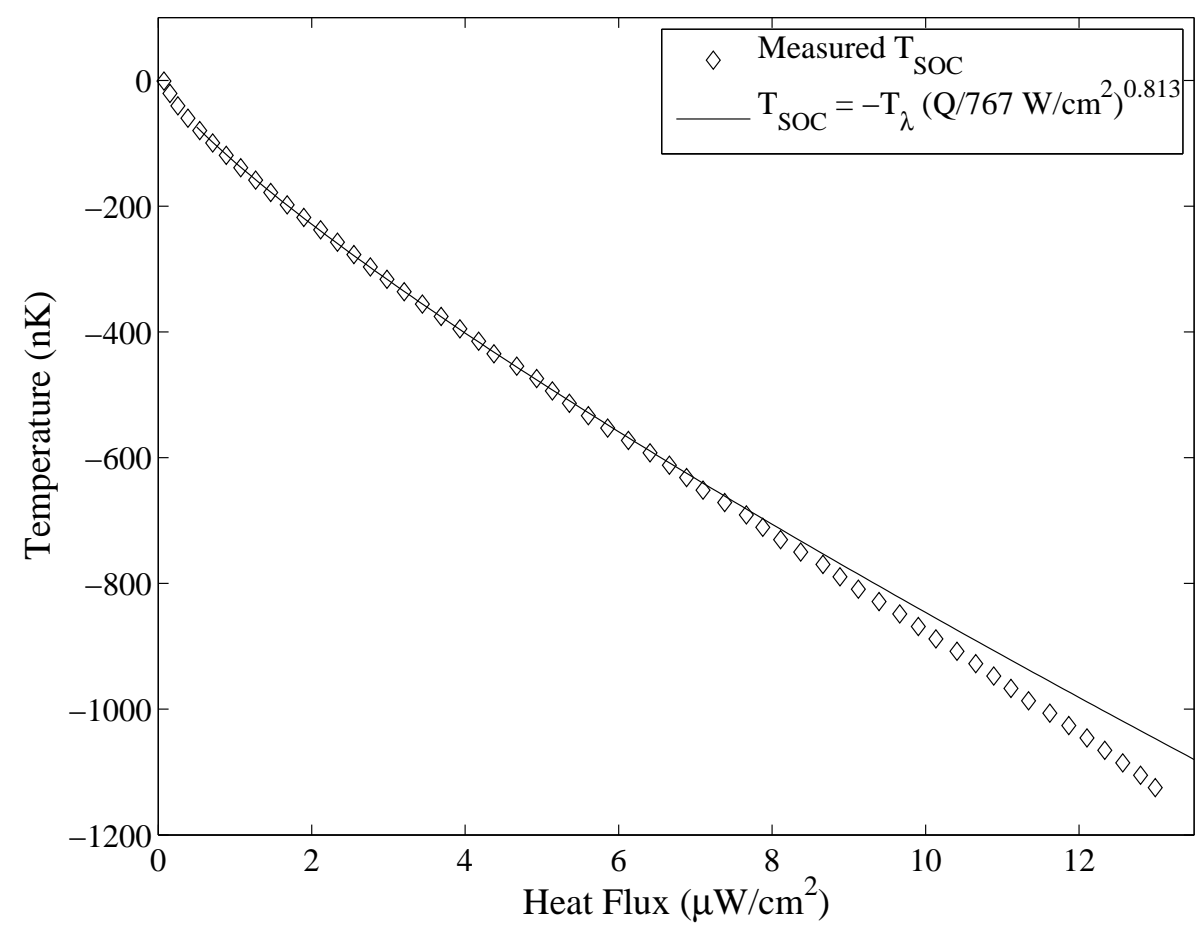

Figure 4.6. $T_{\mathrm{SOC}}$ vs. $Q$. For $0.5 \mu \mathrm{W} / \mathrm{cm}^{2}<Q<5 \mu \mathrm{W} / \mathrm{cm}^{2}, t_{\mathrm{SOC}}(Q)=\frac{T_{\lambda}-T_{\mathrm{SOC}}(Q)}{T_{\lambda}}=$ $\left(Q / 767 \mathrm{~W} / \mathrm{cm}^{2}\right)^{0.813} \pm 10^{-9}$.

later.

We estimate the systematic error caused by incorrectly computing the foil heat to be $<0.5 \%$ for $T_{\mathrm{SOC}}<50 \mathrm{nK}$ below $T_{\lambda}$ and $<1 \%$ for $T_{\mathrm{SOC}}<20 \mathrm{nK}$ below $T_{\lambda}$. We did not take data with a different $\gamma$ any closer to $T_{\lambda}$, so it is impossible to estimate the error beyond this point.

\subsection{7 $T_{\text {SOC }}$ vs. $Q$ and Finding $T_{\lambda}$}

In order to compare the SOC heat capacity measurement to the zero-gravity LPE heat capacity results [22], we need to fix an absolute temperature scale (i.e., we need to find $T_{\lambda}$ ). Previous experiments $[1,3]$ have found that for $Q>500 \mathrm{nW} / \mathrm{cm}^{2}, T_{\mathrm{SOC}}$ has had the following functional form

$$
t_{\mathrm{SOC}}(Q)=\frac{T_{\lambda}-T_{\mathrm{SOC}}(Q)}{T_{\lambda}}=\left(\frac{Q}{Q_{0}}\right)^{0.813}
$$

We fixed $T_{\lambda}$ such that, for heat fluxes $0.5 \mu \mathrm{W} / \mathrm{cm}^{2}<Q<5 \mu \mathrm{W} / \mathrm{cm}^{2}$, our results for $T_{\mathrm{SOC}}(Q)$ followed the curve with $Q_{0}=767 \mathrm{~W} / \mathrm{cm}^{2}$. We show a sample of these results in fig. 4.6. In the region of the fit, the temperature does not deviate more than $1 \mathrm{nK}$ from the equation, although it does appear that the fit residuals, graphed in fig. 4.7, have a significant curvature. No choice of exponent and $T_{\lambda}$ completely removes the curvature. The choice of 0.813 for the exponent, then, is 


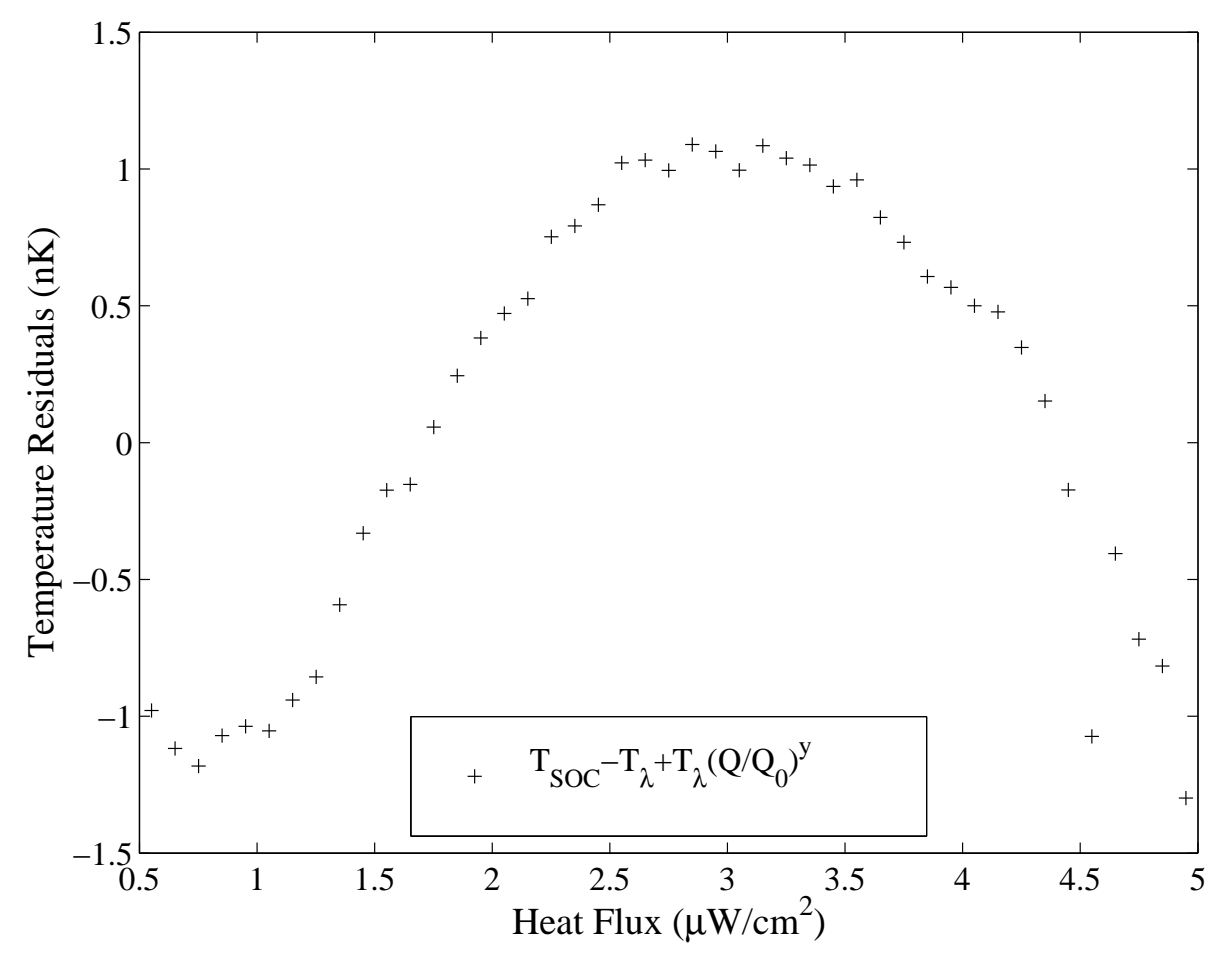

Figure 4.7. $T_{\mathrm{SOC}}$ vs. $Q$ fit residuals. The fit parameters are $Q_{0}=767 \mathrm{~W} / \mathrm{cm}^{2}$ and $x=0.813$.

somewhat arbitrary and its use is primarily convention. For larger heat fluxes, the curvature results in a significant temperature difference, reaching $80 \mathrm{nK}$ at $13 \mu \mathrm{W} / \mathrm{cm}^{2}$. At these high heat fluxes, mutual friction results in a large temperature gradient. If the resistance is large enough, the SOC state can self organize on the changing mutual friction thermal resistance instead of the crossover from superfluid to normal fluid flow. Baddar et al. measured the resistance due to mutual friction to be $R=\left(t /\left(Q / 393 \mathrm{~W} / \mathrm{cm}^{2}\right)^{0.904}\right)^{-2.8}$ [23]. Using our value for $t_{\mathrm{SOC}}(Q)=(Q / 767)^{0.813}$, we find that mutual friction gives a gradient of $1.273 \mu \mathrm{K} / \mathrm{cm}$ for $t_{\mathrm{SOC}}(Q)$ at $Q=20 \mu \mathrm{W} / \mathrm{cm}^{2}$. However, a later experiment done in a heat from above configuration found a significantly higher thermal resistance in the superfluid [3]. Therefore, it is possible that the significant deviation from the exponential fit that we observe starting at $Q \simeq 7 \mu \mathrm{W} / \mathrm{cm}^{2}$ could be caused by mutual friction.

In order to confirm that our choice of fixing $T_{\lambda}$ in this manner is a reasonable thing to do, we measured $T_{\lambda}$ directly. We applied a small amount of heat to the foil heater ${ }^{7}$ and, with no heat flux applied to the top heater, we allowed the cell to slowly ramp up in temperature. While there is superfluid between the top and midplane HRTs, the thermometers tracked one another. However, once the temperature at the midplane passes $T_{\mathrm{C}}$ for our small heat flux, the midplane thermometer increased in temperature quickly compared to the top. We recorded the temperature of the top thermometer at this point. We then measured $T_{\mathrm{SOC}}$ for a heat flux of $1.01 \mu \mathrm{W} / \mathrm{cm}^{2}$ and compared

\footnotetext{
${ }^{7}$ The heat applied created a heat flux through the midplane foil into the helium of $35 \mathrm{nW} / \mathrm{cm}^{2}$.
} 


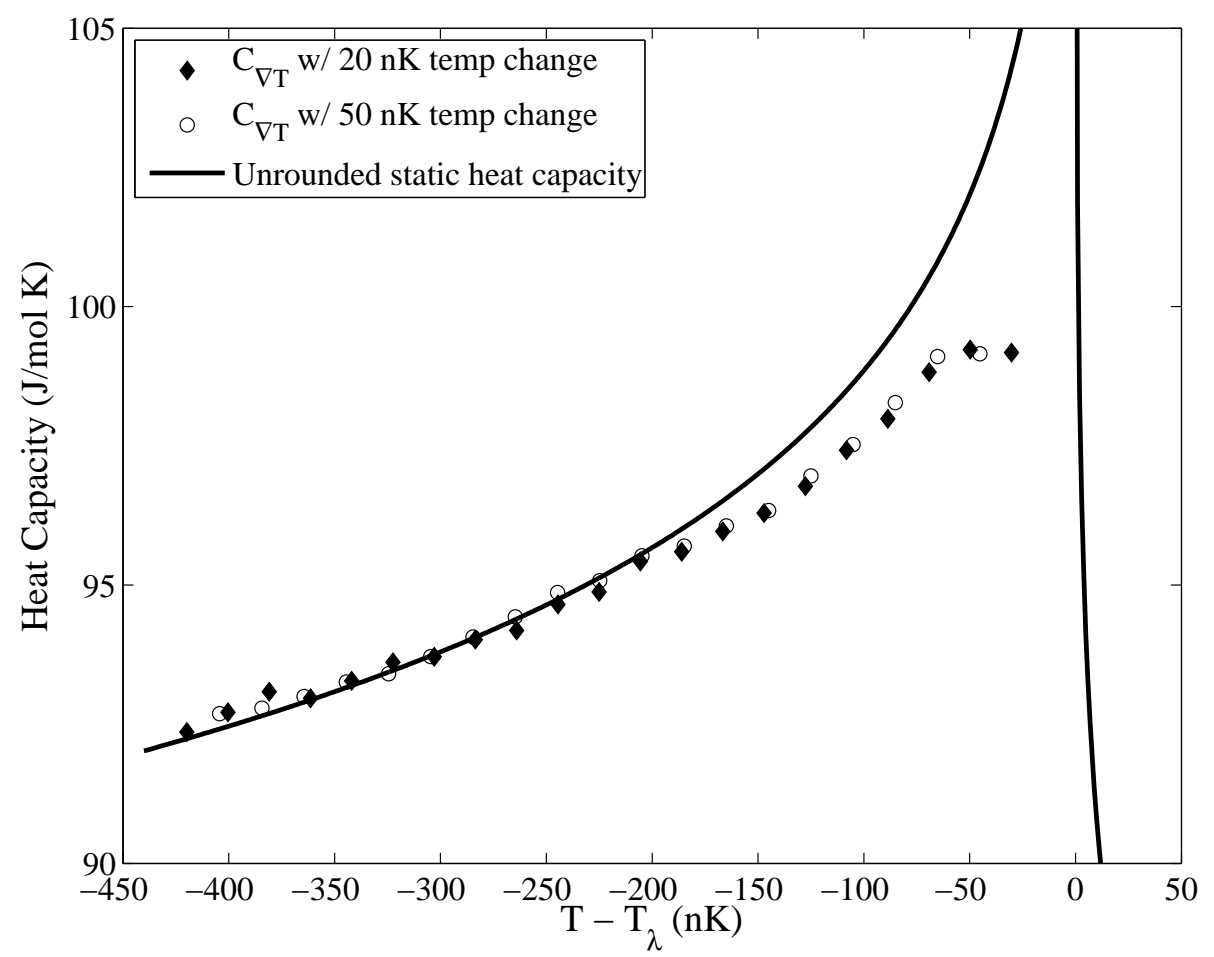

Figure 4.8. SOC heat capacity with different temperature steps $\Delta T_{\mathrm{SOC}}$.

its value to our measured $T_{\mathrm{C}}$. Using this method, we found $T_{\mathrm{SOC}}\left(1.01 \mu \mathrm{W} / \mathrm{cm}^{2}\right)=136 \mathrm{nK}$. Using eq. (4.5), $T_{\mathrm{SOC}}\left(1.01 \mu \mathrm{W} / \mathrm{cm}^{2}\right)=132 \mathrm{nK}$. For our purposes, this is close enough.

\subsubsection{Effect of Different Experimental Parameters}

There are two significant experimental parameters which could effect the outcome of the measurement. The first is the size of the heat flux change from $Q_{1}$ to $Q_{2}$. This change was adjusted to always give the same approximate $\Delta T_{\mathrm{SOC}}$ for each run. Fig. 4.8 shows two data sets, one taken with $50 \mathrm{nK}$ temperature steps and the other taken with $20 \mathrm{nK}$ steps. The second experimental parameter is the ramping heat. Fig. 4.9 shows two sets of results, one taken with $50 \mathrm{nW}$ ramping heat and the other with $20 \mathrm{nW}$. As can be seen from these graphs, changing the experimental parameters has no discernible effect on the results within the range tested.

\subsubsection{Maintaining the Balance, Revisited}

As discussed in sec. 4.1.2, our procedure works best when the heat extracted from the bottom of the SOC state is the same as the heat applied through the top heaters, excluding the $\delta H$ ramping heat that is alternately added then removed. When this is the case, the system is properly balanced and the two time intervals $\tau_{1}$ and $\tau_{2}$ are equal. Fig. 4.10 shows the size of the error when this is not the 


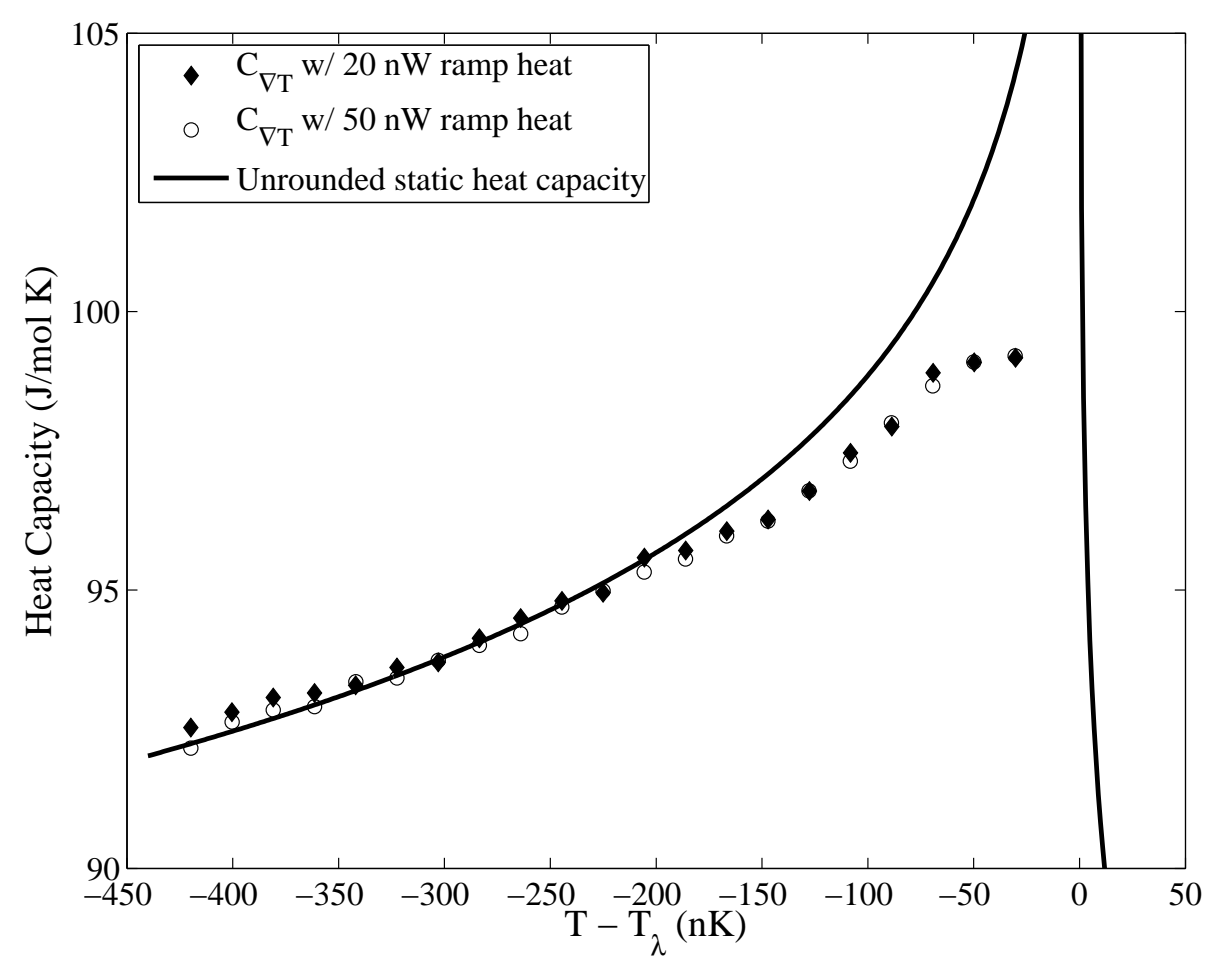

Figure 4.9. SOC heat capacity with different ramping heats $\delta H$.

case. Only data that satisfies the requirement $\left|\tau_{1}-\tau_{2}\right| / \min \left(\tau_{1}, \tau_{2}\right)<0.07$ is averaged to give the balanced results (filled diamonds). All the data is included in the second set of results (open circles) which gives a higher heat capacity close to $T_{\lambda}$. In this section, we will estimate the heat capacity error when the experiment is out of balance and show why a lack of balance results in an upward bias in the heat capacity. We will then calculate a correction that we will apply to all the results reported in this thesis. 


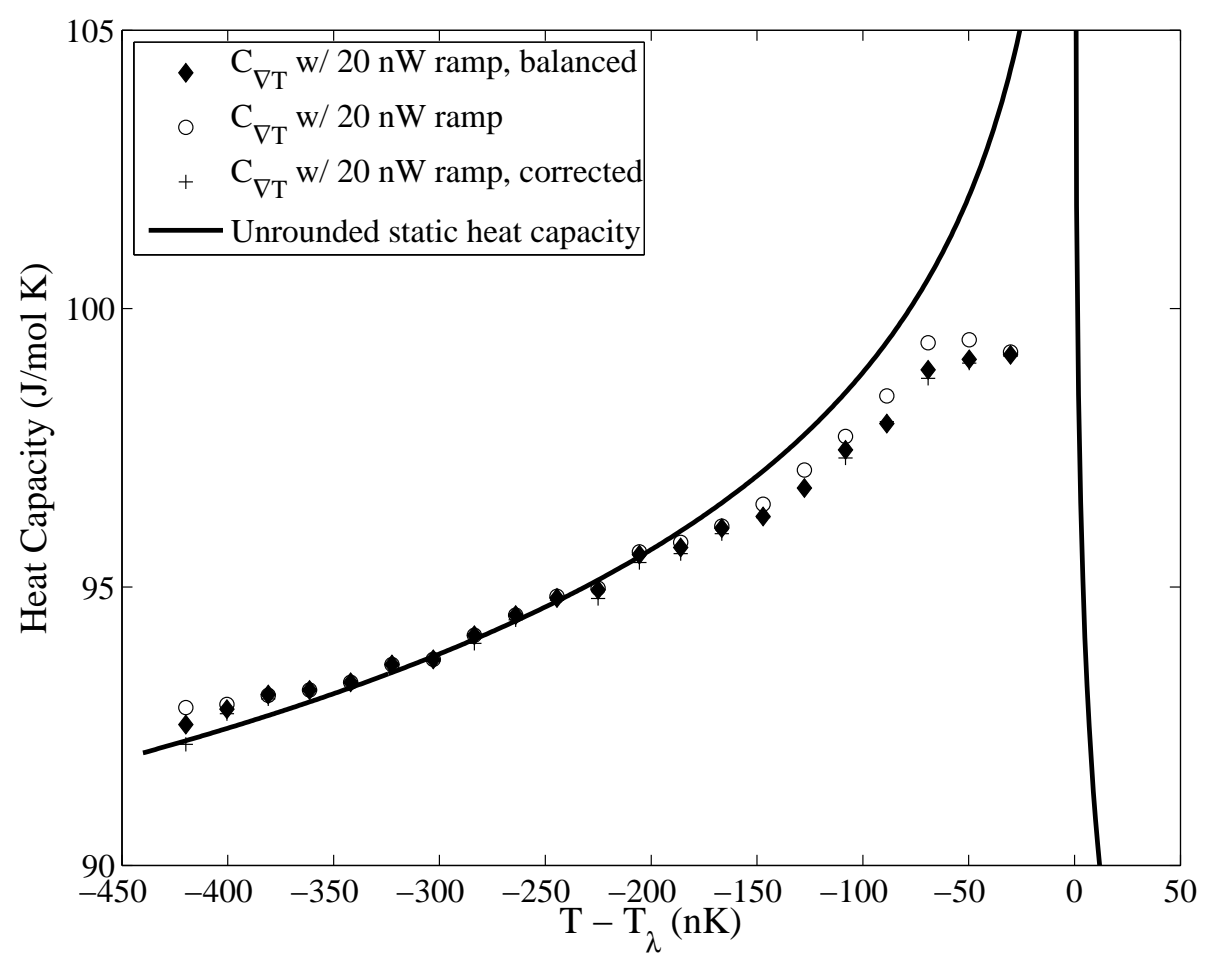

Figure 4.10. The effect of imbalance on the SOC heat capacity. The balanced data include only those measurements where $\left|\tau_{1}-\tau_{2}\right| / \min \left(\tau_{1}, \tau_{2}\right)<0.07$.

When we extract the heat flux $Q_{1}$ and $Q_{2}$ through the SOC state, we are never perfectly balanced and actually apply $Q_{1} \times A+\alpha \pm \delta H$ and $Q_{2} \times A+\beta \pm \delta H$ with the top heaters, where $\alpha$ and $\beta$ are errors and hopefully small, $A$ is the cell endplate area, and $\delta H$ is the ramping heat. We will assume that $\alpha=\beta$ for this calculation. ${ }^{8}$ Given this assumption, the true heat capacity is $C=(\delta H+\alpha) \tau_{1} / \Delta T$. If we only look at the first half our experimental procedure $\left(Q_{1} \rightarrow Q_{2}\right)$ we would have an error of $\Delta C / C=\alpha /(\delta H+\alpha)$. Since we perform both halves of the experiment $\left(Q_{1} \rightarrow Q_{2}\right.$ and $\left.Q_{2} \rightarrow Q_{1}\right)$, we can do a lot better. In this case, the true heat capacity is

$$
\begin{aligned}
C & =\frac{(\delta H+\alpha) \tau_{1}+(\delta H-\alpha) \tau_{2}}{2 \Delta T} \\
& =\frac{\delta H\left(\tau_{1}+\tau_{2}\right) / 2}{\Delta T}+\frac{\alpha\left(\tau_{1}-\tau_{2}\right) / 2}{\Delta T} .
\end{aligned}
$$

Our computed heat capacity is given by the first term (see eq. (4.4)) and the second term is the error. Now, $\alpha$ and the time intervals $\tau_{1}$ and $\tau_{2}$ are related by

$$
(\delta H+\alpha) \tau_{1}=(\delta H-\alpha) \tau_{2}
$$

\footnotetext{
${ }^{8}$ We performed a numerical analysis and found that for $\alpha \neq \beta$, the correction computed here is within $10 \%$ of the true value for our data. This is more than sufficient for our needs because the correction itself is less than $1 \%$ of the total heat capacity.
} 


$$
\alpha=\frac{\delta H\left(\tau_{2}-\tau_{1}\right)}{\tau_{2}+\tau_{1}} .
$$

Using eq. (4.6) and (4.7), we compute that our heat capacity results are biased upwards from the true heat capacity by

$$
\frac{\Delta C}{C}=\left(\frac{\tau_{1}-\tau_{2}}{\tau_{1}+\tau_{2}}\right)^{2} .
$$

For the the third set of results (plus signs) in fig. 4.10, we have applied this correction, and it removes the upward bias in the heat capacity. Therefore, we have applied this correction to all the SOC heat capacity results reported elsewhere in this thesis. For the results to be discussed in our conclusion, however, the experiment was never as far out of balance as in this particular data set. The correction only resulted in a visible (i.e., $>0.5 \%$ ) change for the data below $1.09 \mu \mathrm{K}$ below $T_{\lambda}$ where the heat capacity was corrected downwards by almost $1 \%$.

\subsection{Results and Conclusion}

\subsubsection{Results}

The SOC heat capacity results $\left(C_{\nabla T}\right)$ for our two main data runs are shown in fig. 4.11. Table 4.1 lists the parameters for the two data sets. The data are binned into $10 \mathrm{nK}$ temperature increments and are averaged by discarding the top and bottom quarter and averaging the remaining points in the bin. Any bins that have fewer than four points, however, are ignored. The data are compared to the micro-gravity results from the NASA Lambda Point Experiment (LPE).

Table 4.1. Parameters for SOC heat capacity runs

\begin{tabular}{rccc} 
Minimum $Q$ & Maximum $Q$ & $\delta H$ & $\Delta T$ \\
\hline $45.5 \mathrm{nW} / \mathrm{cm}^{2}$ & $4.56 \mu \mathrm{W} / \mathrm{cm}^{2}$ & $35 \mathrm{nW}$ & $20 \mathrm{nK}$ \\
$84 \mathrm{nW} / \mathrm{cm}^{2}$ & $13.3 \mu \mathrm{W} / \mathrm{cm}^{2}$ & $45 \mathrm{nW}$ & $25 \mathrm{nK}$
\end{tabular}

For $600 \mathrm{nK}<T_{\mathrm{SOC}}<250 \mathrm{nK}$ below $T_{\lambda}$, the measured SOC heat capacity equals the zero gravity results from LPE. In this way, the SOC state acts as zero gravity simulator. Without the effects of SOC state, our $2.54 \mathrm{~cm}$ tall cell would have $3 \mu \mathrm{K}$ of gravity rounding.

Both sets of results show a significant depression in the heat capacity near $T_{\lambda}$. The set with $Q_{\max }=4.56 \mu \mathrm{W} / \mathrm{cm}^{2}$ is more trustworthy at high temperatures. There were more than twice the number of points per bin, there was a lower sidewall heat leak, and we took data at warmer temperatures. However, all the data above $50 \mathrm{nK}$ below $T_{\lambda}$ are susceptible to the possible systematic error in finding the signature of the fully formed SOC state discussed at the end of sec. 4.2.3. As discussed in sec. 4.2.6, results for $T_{\mathrm{SOC}}(Q)>50 \mathrm{nK}$ below $T_{\lambda}$ are also susceptible to a systematic error from the midplane foil heat leak. We estimate that the total systematic error is less than $0.7 \%$ 


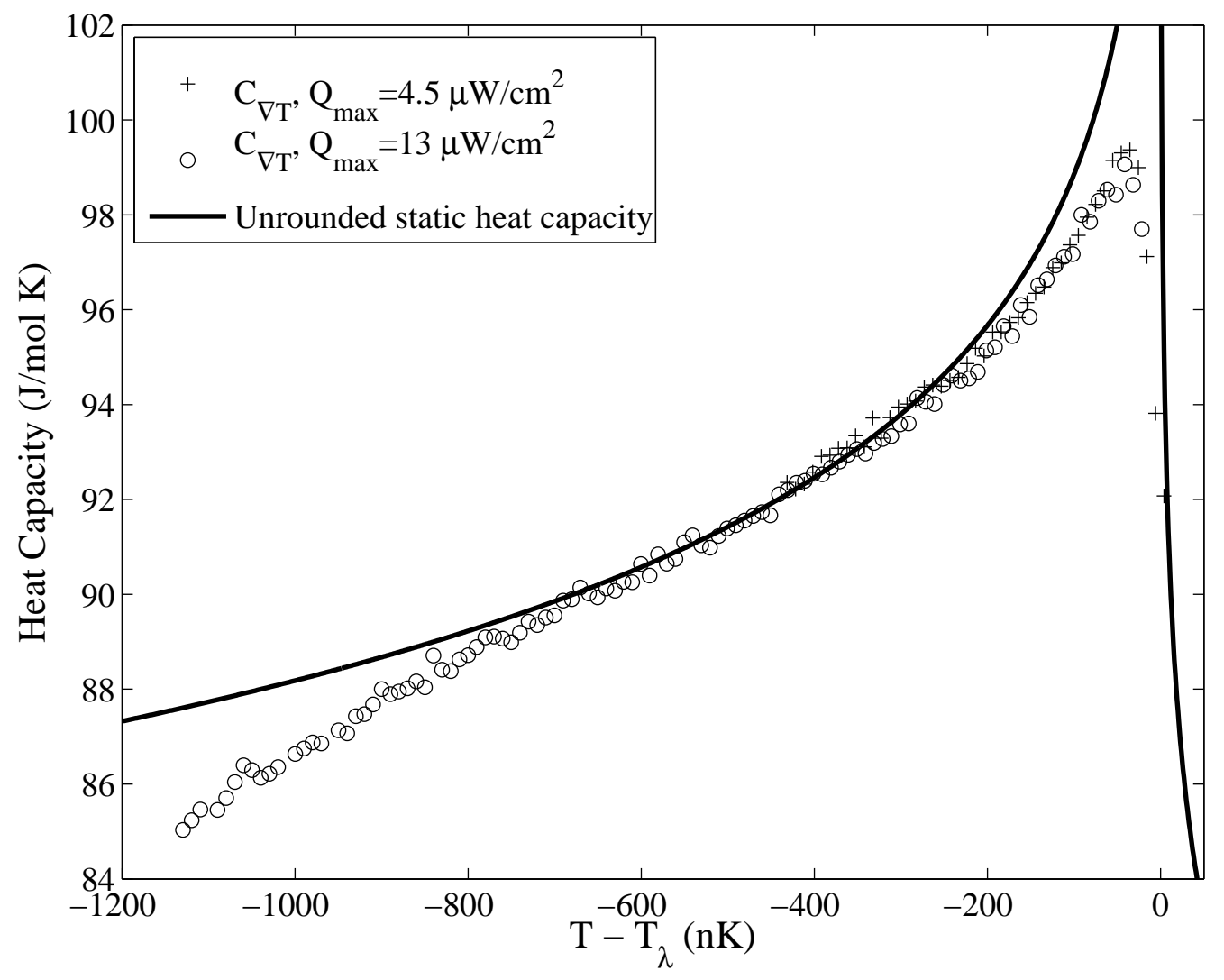

Figure 4.11. SOC heat capacity. 


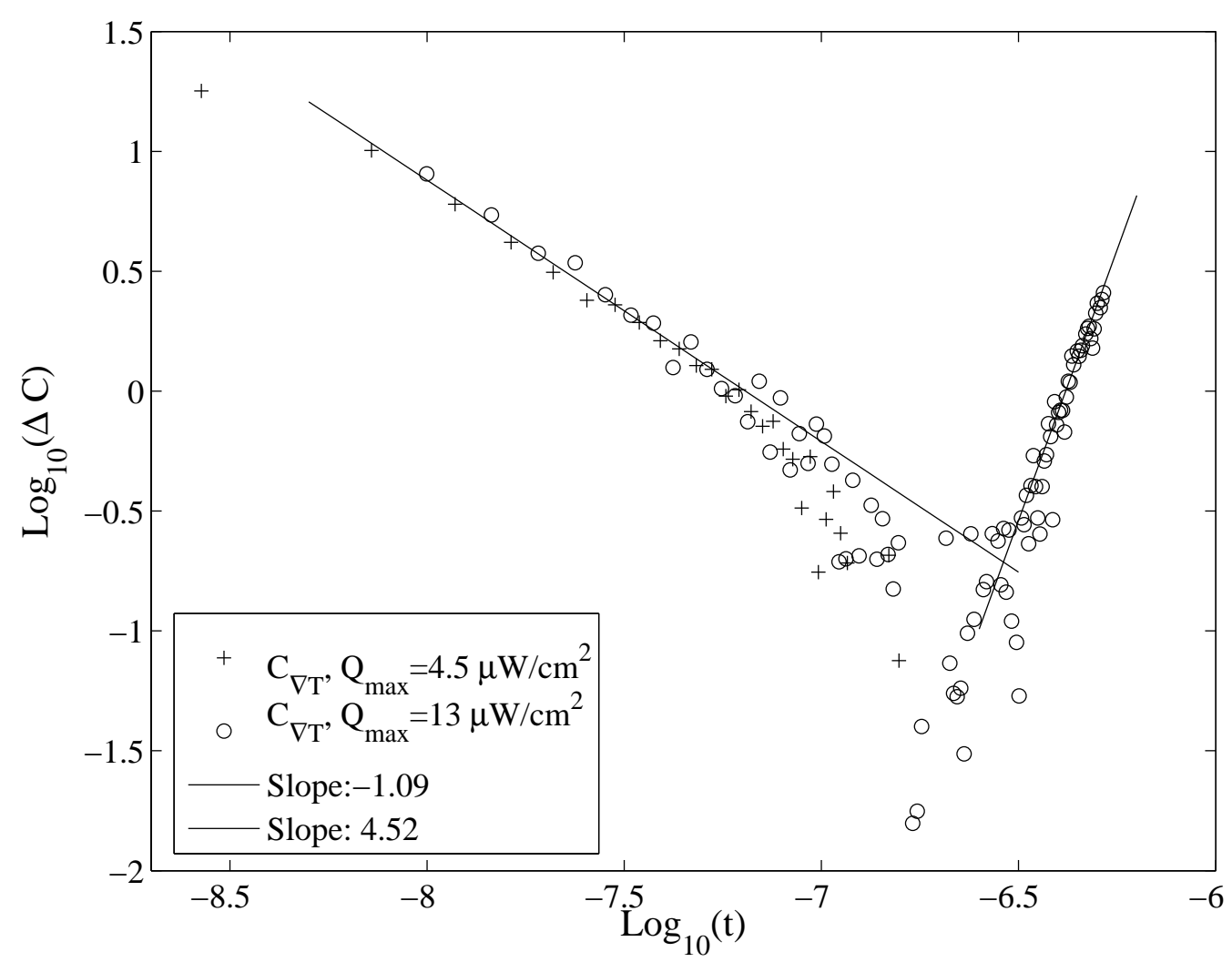

Figure 4.12. Log-log plot of SOC heat capacity.

for $T_{\mathrm{SOC}}<50 \mathrm{nK}$ below $T_{\lambda}$ and less than $1.5 \%$ for $T_{\mathrm{SOC}}<30 \mathrm{nK}$. For the remainder of the results, we estimate that our total systematic errors are less than $4 \%$, but we do not have sufficient data to be completely confident in this estimate.

At very low temperatures and high heat fluxes, there is also a significant depression in the heat capacity. This occurs over the same region where $T_{\mathrm{SOC}}(Q)$ no longer follows the exponent 0.813 , as shown in sec. 4.2.7, which is the same region where we believe that the resistance due to mutual friction becomes significant.

We can see the heat capacity depression more clearly if we plot $\Delta C=C-C_{\nabla \mathrm{T}}$ vs. the reduced temperature $t=\frac{T_{\lambda}-T}{T_{\lambda}}$ on a log-log plot, as shown in fig. 4.12. Near $T_{\lambda}$ (and at low heat flux), $\Delta C \sim t^{-1.1}$. At low temperatures (and at high heat flux), $\Delta C \sim t^{4.5}$.

\subsubsection{Comparison with Theory}

Rudolf Haussmann used Dynamic Renormalization Group (DRG) theory to calculate the SOC state heat capacity[9]. He predicted a depression near $T_{\lambda}$. We plot our results with his prediction in fig. 4.13 to compare the size of the depression. We have scaled our data upwards by $0.13 \%$ in order to match his static heat capacity with ours. In addition, we plot the results of a more recent Haussmann 


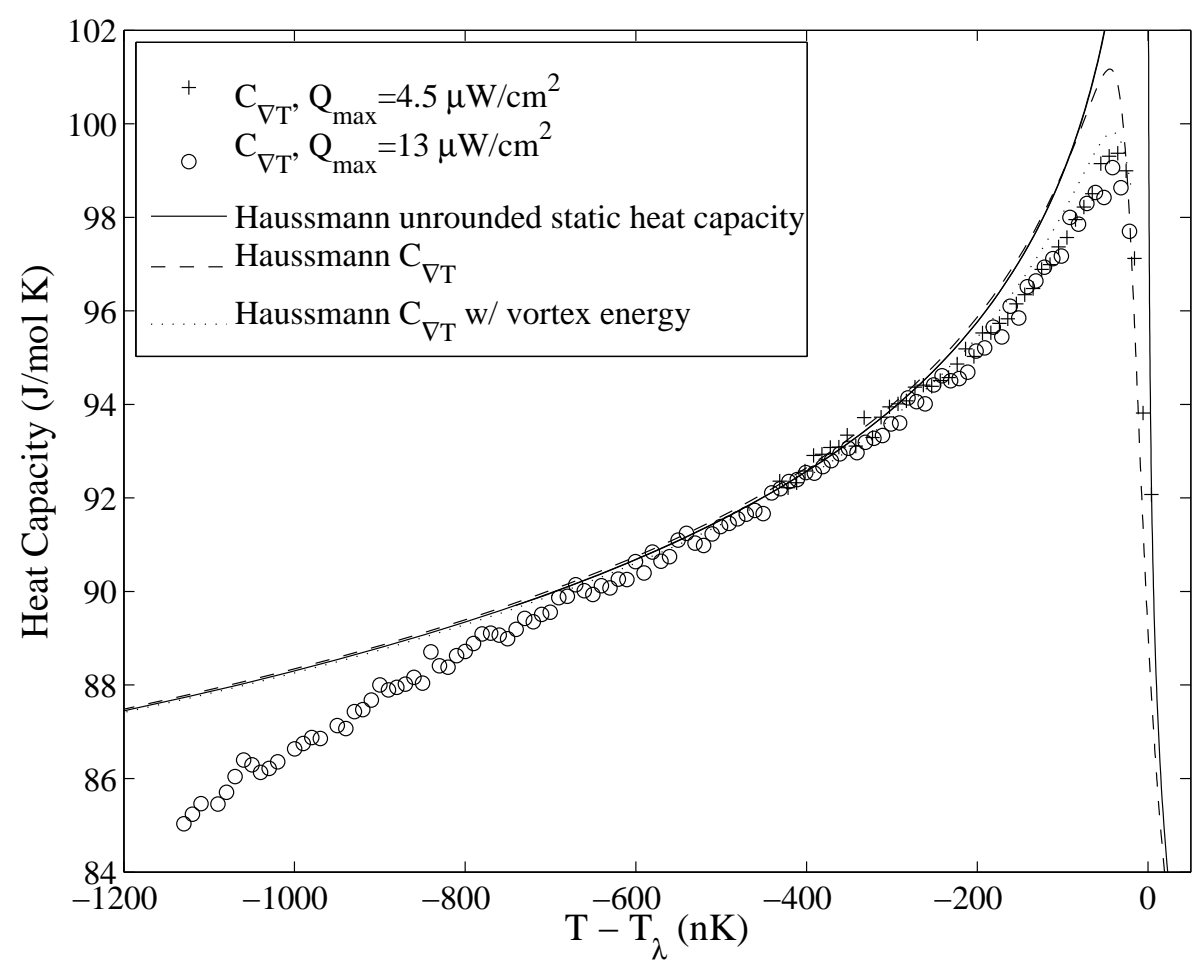

Figure 4.13. SOC heat capacity results compared to Haussmann's prediction.

calculation that includes an estimate for the vortex core energy [24]. The new calculation includes a parameter that is not derived from the theory for which Haussmann has inserted a reasonable number. As of yet, he has not used the parameter to attempt to fit our results. We are only showing the results near $T_{\lambda}$ because both of Haussmann's predictions show no depression at high heat flux. In fig. 4.14, we plot Haussmann's prediction with the vortex energy parameter with our data on a $\log -\log$ plot.

\subsubsection{Conclusion}

Although we see deviations from the static heat capacity, the differences are relatively small and the general structure of a heat capacity maximum near $T_{\lambda}$ is maintained. Moeur et al. modeled the SOC state as a normal fluid with a thermal conductivity given by eq. (1.16). It is tempting to conclude that $T_{\mathrm{C}}(Q)$ is a depressed critical point $T_{\lambda}(Q)$ and the entirety of the SOC state exists in the normal fluid. The helium in the SOC state has a gradient and the thermal conductivity has an apparent divergence at $T_{\mathrm{C}}(Q)$. Despite this evidence, the heat capacity results show that the phase transition occurs much like the static case near $T_{\lambda}$, although the transition is significantly rounded.

At low heat flux, we see a departure from the static heat capacity that is significantly larger than Haussmann's original DRG prediction. However, the location of the maximum in both theory 


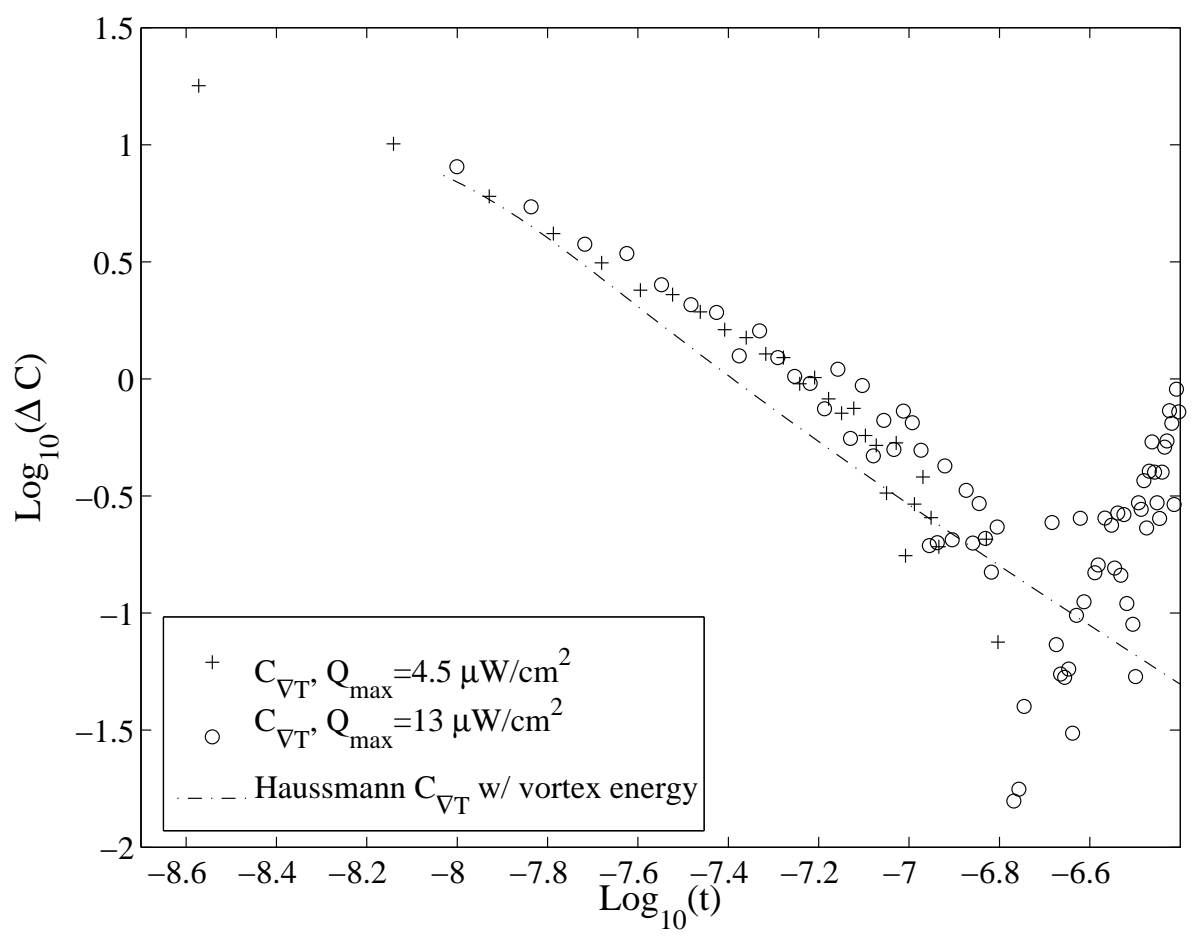

Figure 4.14. Log-log plot of SOC heat capacity results compared to Haussmann's prediction.

and experiment is $50 \mathrm{nK}$ below $T_{\lambda}$. Haussmann's second prediction, which includes the effect of the energy of vortex cores, is a much closer match to our results.

At higher heat flux, our measurement of $C_{\nabla T}$ is significantly depressed compared to the static heat capacity and Haussmann's prediction. At these heat fluxes, $Q>7 \mu \mathrm{W} / \mathrm{cm}^{2}$, the resistance caused by mutual friction becomes significant. It is possible that the proliferation of vortices interacting with the normal component of the superfluid is sufficient to suppress the random fluctuations that contribute to the heat capacity. 


\section{Chapter 5}

\section{The SOC Wave}

While our apparatus was designed explicitly to measure the heat capacity of the SOC state, it is reasonably well suited to making measurements of the SOC wave discussed in sec. 1.3. Steve Boyd and Rob Duncan at UNM designed a measurement procedure for determining the speed of the SOC wave that does not need two sidewall thermometers [25]. As we will see, it does not even need one sidewall thermometer. We can measure the wave properties over our range of heat fluxes, which span temperatures both above and below $T_{\lambda}$, and look for some evidence of the phase transition. In addition, we can compare our results to the predictions given in the introduction.

\subsection{Procedure}

The procedure starts with a heat flux $Q$ flowing downwards through the helium with the helium in the superfluid state, just below $T_{\mathrm{SOC}}(Q)$ at the bottom of the sample. A sinusoidal heat $A \sin (2 \pi f t)$ is added to the bottom heater. ${ }^{1}$ The amplitude is chosen so that a readable signal of approximately $1 \mathrm{nK}$ peak to peak can be extracted from the top thermometer. (We have an integrated heat of $\frac{A}{f \pi}$, so $A \approx 0.5 \mathrm{nK} f \pi C \approx 47 f \mathrm{~nJ}$.) The top heater is then increased to $Q \times A+\delta H$ and the cell is allowed to slowly warm.

Initially, when the helium is entirely superfluid, there should be approximately $\pi / 2$ phase shift between the application of heat to the bottom endplate and the response of the helium. The bottom endplate, which has a very small heat capacity and moderate resistance to the helium, responds almost instantly $(\tau \sim 3 \mathrm{~ms}$ ) to reach the temperature such that the heat flux exiting the endplate into the helium equals the amount we are applying through the heater. The helium's temperature response is proportional to the integral of the heat flux, which gives the $\pi / 2$ phase shift. As we let the helium warm, the SOC state begins to form at the bottom of the cell. As the wave travels through the SOC layer, a phase delay is created that is proportional to the height of the layer. We continue to let the sample warm until the SOC state fills most of the cell, then cool down and repeat

\footnotetext{
${ }^{1}$ We apply the necessary voltage output in order to ensure that the heat output is sinusoidal.
} 


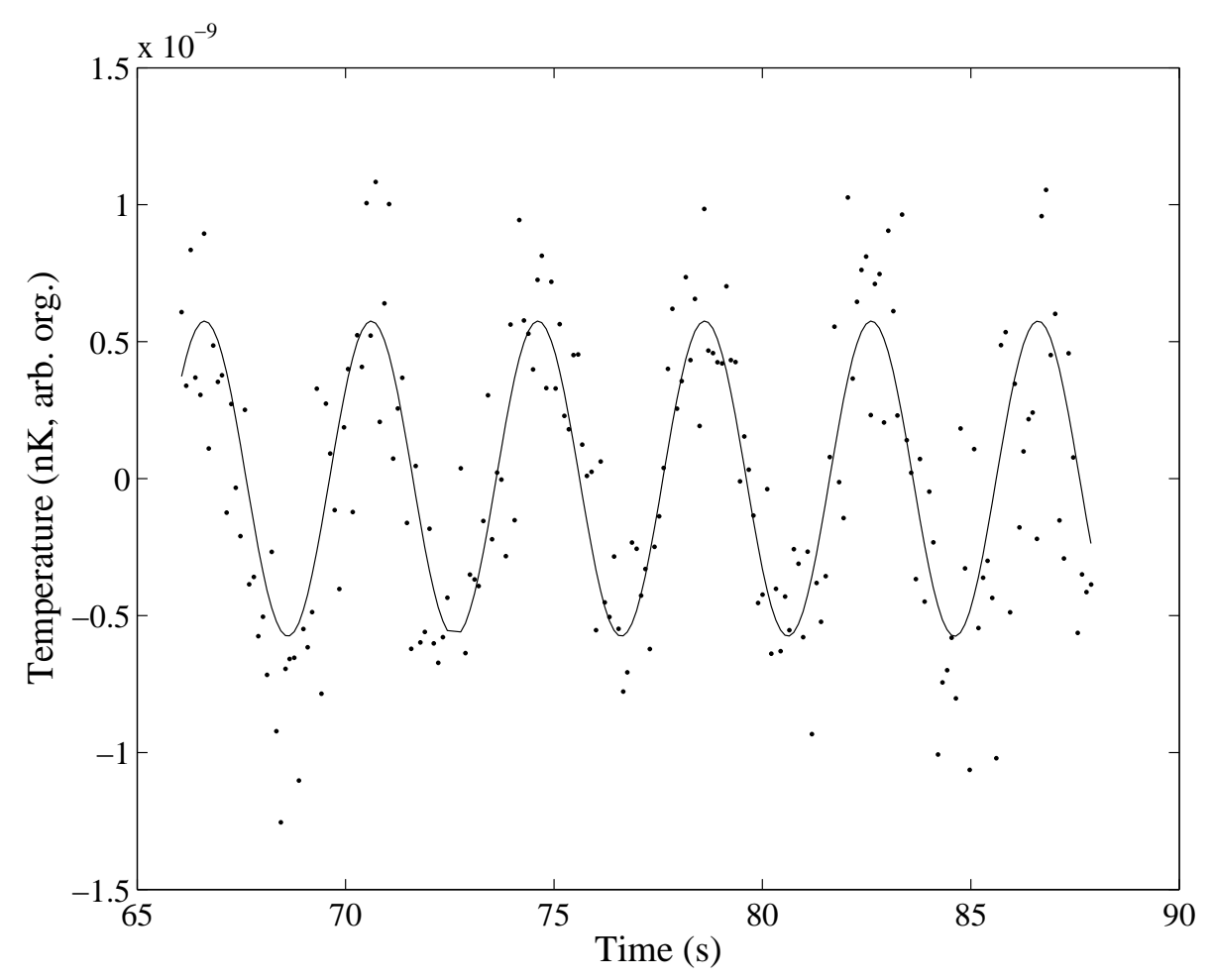

Figure 5.1. Comparison of $\mathrm{HRT}_{\mathrm{T}}$ to a reference sine wave. The linear drift of $\mathrm{HRT}_{\mathrm{T}}$ has been removed and the reference sine wave has already been scaled and shifted to match the data.

the procedure with a different heat flux.

\subsection{Analysis}

\subsubsection{Phase and Amplitude}

The temperature data for the three thermometers, $\mathrm{HRT}_{\mathrm{B}}, \mathrm{HRT}_{\mathrm{M}}$, and $\mathrm{HRT}_{\mathrm{T}}$, are partitioned into segments between 20 to $200 \mathrm{~s}$ long, depending on the data file. For each data segment for each thermometer, phase and amplitude is extracted as follows. The temperature segment is fit to a straight line. The residuals from this fit, $R_{i}$ along with time data $t_{i}$, are compared to a reference sine wave with a variable phase, $\phi$. A plot of such a comparison is shown in fig. 5.1 where we have already scaled the amplitude of the reference wave. We vary $\phi$ until we find the maximum of

$$
A=\frac{2}{N} \sum_{i} R_{i} \sin \left(2 \pi f t_{i}-\phi\right)
$$

The values $\phi_{\max }$ and $A_{\max }$ give the phase and amplitude of the data segment. Since the function $A(\phi)$ is a sine wave itself, it is extremely difficult to pinpoint the correct phase just using the 


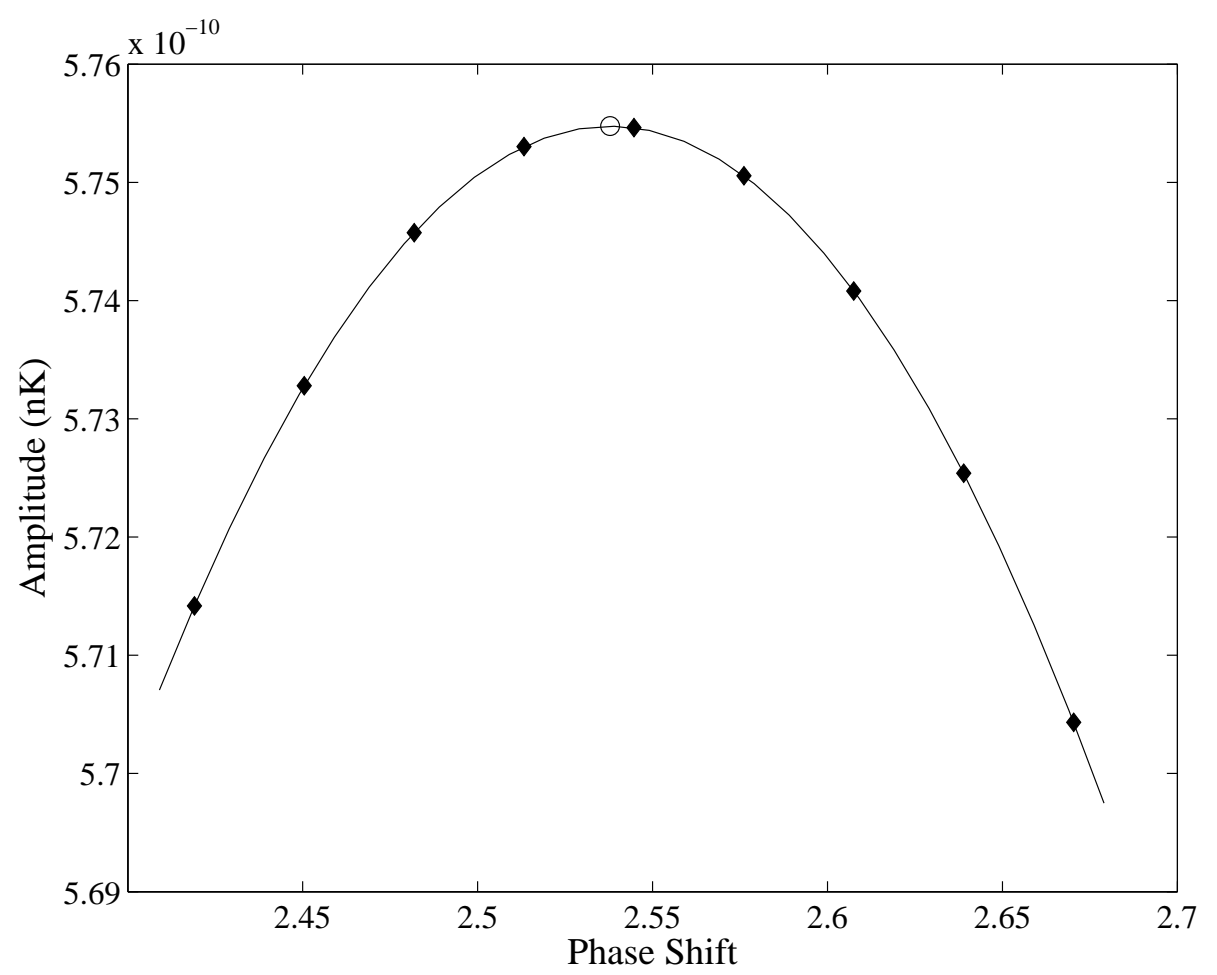

Figure 5.2. Amplitude vs. reference sine wave phase shift. The results for the tested phase shifts are fit to a quadratic and the maximum of the fit is marked with a circle.

maximum (i.e., the peak of the curve is flat and rounded). Therefore, we do a quadratic fit in the region of the maximum to get better precision. The results of such a fit are shown in fig. 5.2. The location of the maximum is marked by a circle. To get the phase delay of the application of heat to the thermometer response, we subtract the phase of $\mathrm{HRT}_{\mathrm{B}}$ from $\mathrm{HRT}_{\mathrm{T}}$ and $\mathrm{HRT}_{\mathrm{M}}$. A plot of the measured phase vs. $\mathrm{HRT}_{\mathrm{T}}$ is given in fig. 5.3.

There is one complication. The measured phase delay includes the delay as the wave travels through the SOC state as well as numerous other delays. There are the response times of the HRTs as well as the $R C$ time constant of the bottom and top endplates. Since these delays are relatively constant, they can be ignored. However, the delay from the speed of second sound through the superfluid changes as the thickness of the SOC layer grows. In our region near $T_{\lambda}$, the speed of second sound [26] is

$$
u_{2}=\sqrt{\frac{\rho_{s} T S^{2}}{\rho C}} \sim 2460 t^{0.335} \mathrm{~cm} / \mathrm{s}
$$




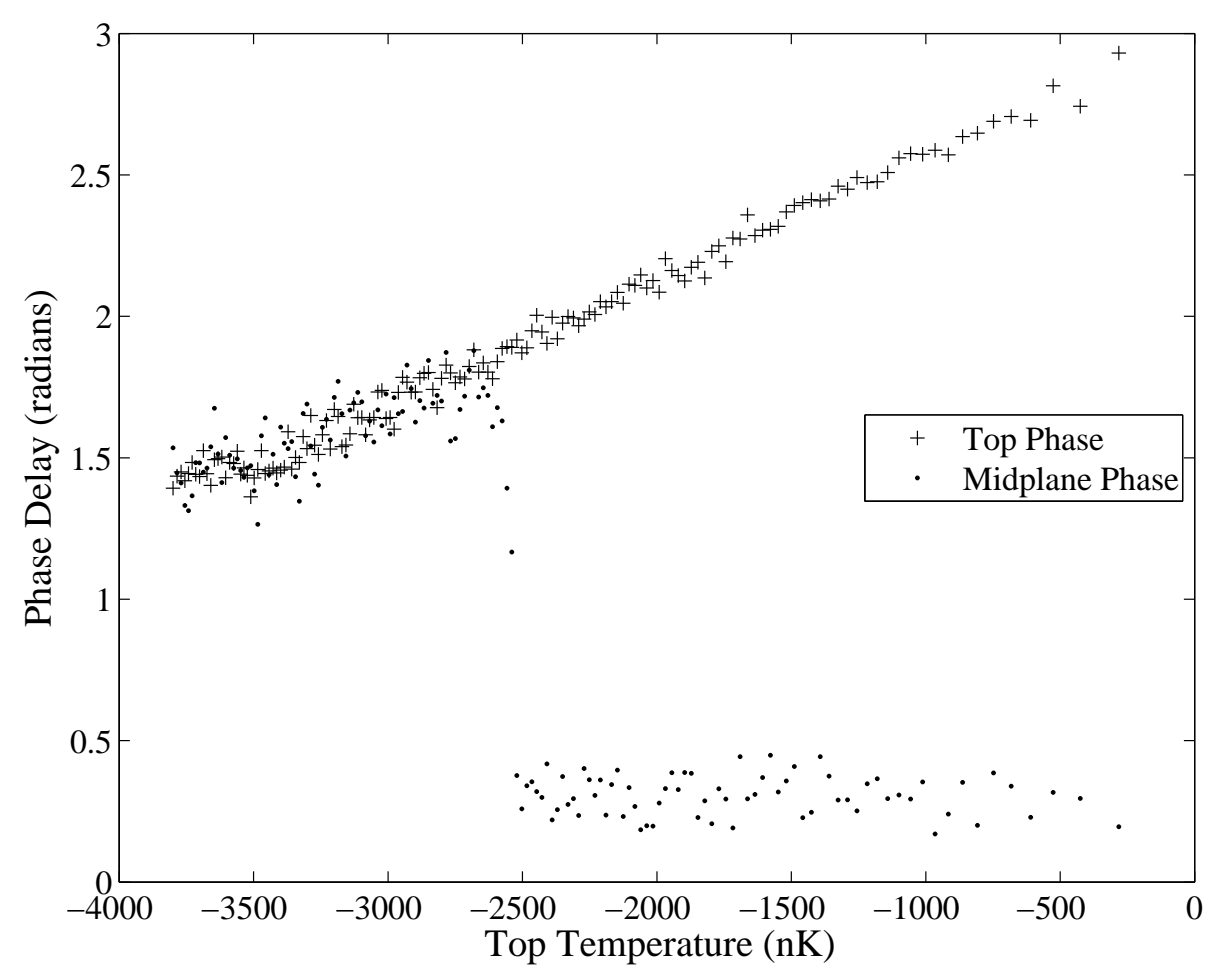

Figure 5.3. HRT $_{\mathrm{T}}$ phase delay and $\mathrm{HRT}_{\mathrm{M}}$ phase delay vs. $\mathrm{HRT}_{\mathrm{T}} \cdot \mathrm{HRT}_{\mathrm{M}}$ sees a $\pi / 2$ drop in the delay when the SOC state crosses the midplane. At this point, the thermometer is measuring the wave itself, rather than the integrated response of the helium above the SOC state.

where $\rho$ is the density of the helium, $\rho_{s}$ is the density of the superfluid portion, $T$ is the temperature, $S$ is the entropy, $C$ is the heat capacity, and $t=\left(T_{\lambda}-T\right) / T_{\lambda}$ is the reduced temperature. This speed ranges from $4 \mathrm{~cm} / \mathrm{s}$ at $10 \mathrm{nK}$ to $18 \mathrm{~cm} / \mathrm{s}$ at $1 \mu \mathrm{K}$ below $T_{\lambda}$. The time delay for the wave to reach the top of the helium is given by

$$
\begin{aligned}
\tau & =\int_{z_{1}}^{z_{2}} \frac{d z}{u_{2}} \\
& =\frac{2.1768 \mathrm{~K}}{1.273 \mu \mathrm{K} / \mathrm{cm}} \int_{t_{1}}^{t_{2}} \frac{d t}{2460 t^{0.335}} \\
& =1045 \mathrm{~s}\left[t^{0.665}\right]_{t 1}^{t 2}
\end{aligned}
$$

In a typical scenario the reduced temperature at the bottom of the cell is $10^{-7}$, which would give a total time delay of $\tau=122 \mathrm{~ms}$. If this were a constant delay, we could ignore this effect. However, as the SOC layer grows, the superfluid layer shrinks, and the second sound delay will get smaller.

We compute the second sound delay using eq. (5.3). The integral start temperature, $t_{1}$, is arbitrary. $^{2}$ The integral end temperature, $t_{2}$, is $\mathrm{HRT}_{\mathrm{T}}$. We provide an absolute calibration for

\footnotetext{
${ }^{2}$ Once the SOC state has formed at the bottom of the cell the start temperature (in reduced temperature) is always $t_{\mathrm{SOC}}(Q)$. Since we are only concerned with the slope of the phase delay curve and not it's absolute value, we are free
} 
$\mathrm{HRT}_{\mathrm{T}}$ by using the measured $T_{\mathrm{SOC}}$ of $\mathrm{HRT}_{\mathrm{M}}$ and making the appropriate adjustment to $T_{\lambda}$ for the height difference. This calibration could easily be off by $50 \mathrm{nK}$, so this computed second sound delay should not be used when such an error would make a significant difference. As long as $\mathrm{HRT}_{\mathrm{T}}$ stays $200 \mathrm{nK}$ from $T_{\lambda}$, the error in the second sound speed would be less than $10 \%$. In addition, second sound becomes nonlinear close to $T_{\lambda}$ or for large oscillations [27]. However, as long as $\mathrm{HRT}_{\mathrm{T}}$ stays $200 \mathrm{nK}$ from $T_{\lambda}$, the error in the second sound speed is less than $5 \%$ for amplitudes up to $10 \mathrm{nK}$. Given the already small size of the correction, these small errors have no noticeable impact on the results.

\subsubsection{SOC Layer Height}

We find the change in layer height using $\Delta h=\Delta \mathrm{HRT}_{\mathrm{T}} \frac{d T_{\lambda}}{d z}$. This is true as long as the helium at the top of the cell has a negligible gradient and the helium at the bottom of the cell is in the SOC state. Otherwise, the relationship of the layer height (and, therefore, the phase delay) will no longer have a linear relationship to the top temperature. Therefore, we limit our investigations to the region of the results where the linear relationship holds. Given our tall cell, this region is over $1.5 \mu \mathrm{K}$ for all heat fluxes.

\subsubsection{Speed}

Ignoring the corrections and complications of the previous sections, the wave speed can be determined from the slope of a plot of the phase delay vs. the top temperature, such as in fig. 5.3. If the slope is $m=\frac{d \phi}{d \mathrm{HRT}_{\mathrm{T}}}$ and the frequency of the excitation is $f$, then the speed is given by

$$
v=\frac{2 \pi f}{m 1.273 \times 10^{-6} \mathrm{~K} / \mathrm{cm}} .
$$

In order to take into account the finite speed of second sound through the superfluid, we subtract the phase delay computed using eq. (5.3) from the total phase delay. Fig. 5.4 shows a plot with the correction applied. We use the arbitrary integration parameter to set the correction equal to zero as the SOC state first enters at the bottom of the sample. We are only concerned with the slope, so this does not affect the answer, but it does allows us to better compare how the slope has changed after the correction. The velocity can then be extracted using the slope of the corrected curve with eq. (5.5). The best fit line is representative of the region of the fit.

In general, the fits to the slope were done from the moment the SOC state entered the sample until the SOC state was about two thirds of the way through the sample. This gave a large enough region to get a good result for the slope while avoiding the errors discussed in sec. 5.2.2. However, there were a couple of circumstances where we had to slightly alter the extent of the fit. For very low

to set it arbitrarily. 


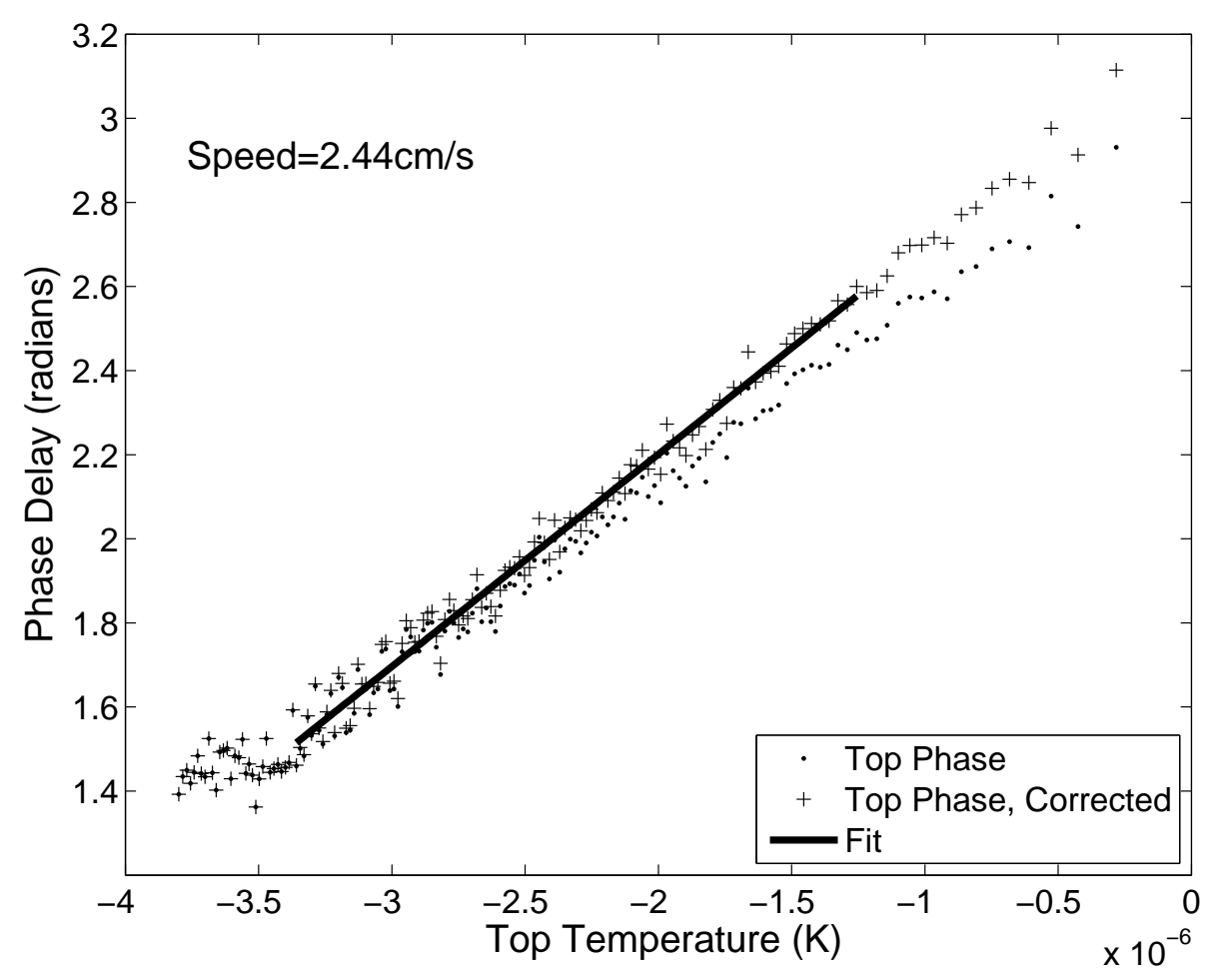

Figure 5.4. $\mathrm{HRT}_{\mathrm{T}}$ phase delay vs. $\mathrm{HRT}_{\mathrm{T}}$ before and after the second sound correction is applied for $Q=1.02 \mu \mathrm{W} / \mathrm{cm}^{2}$. The best fit line is drawn over the region of the fit. The speed is computed from the slope using eq. (5.5).

heat fluxes (below about $80 \mathrm{nW} / \mathrm{cm}^{2}$ ), there was a small crossover region between the flat region in the phase vs. top temperature plot before resistive fluid appeared at the bottom of the sample and the region of constant slope as the SOC state traveled up the sample. In addition to looking for the constant slope, we also looked at the amplitude of the oscillations in the bottom thermometer $\mathrm{HRT}_{\mathrm{B}}$. When the resistive fluid first appeared at the bottom of the sample, the amplitude in $\mathrm{HRT}_{\mathrm{B}}$ grew, but once the SOC state formed, the amplitude remained constant (because the bottom temperature was now self-organized and did not change) thus providing a marker for starting our fit. The second instance where the region of the fit needed to be altered was for waves with large attenuation (i.e., waves of high frequency and low heat flux). In this case, the amplitude of the signal at the top thermometer would get very small and the phase results would become unreliable as the SOC layer grew thicker. In both cases, it was easy to change the limits of the fit to ensure a flat profile in the fit residuals and a reliable indication of the slope. 


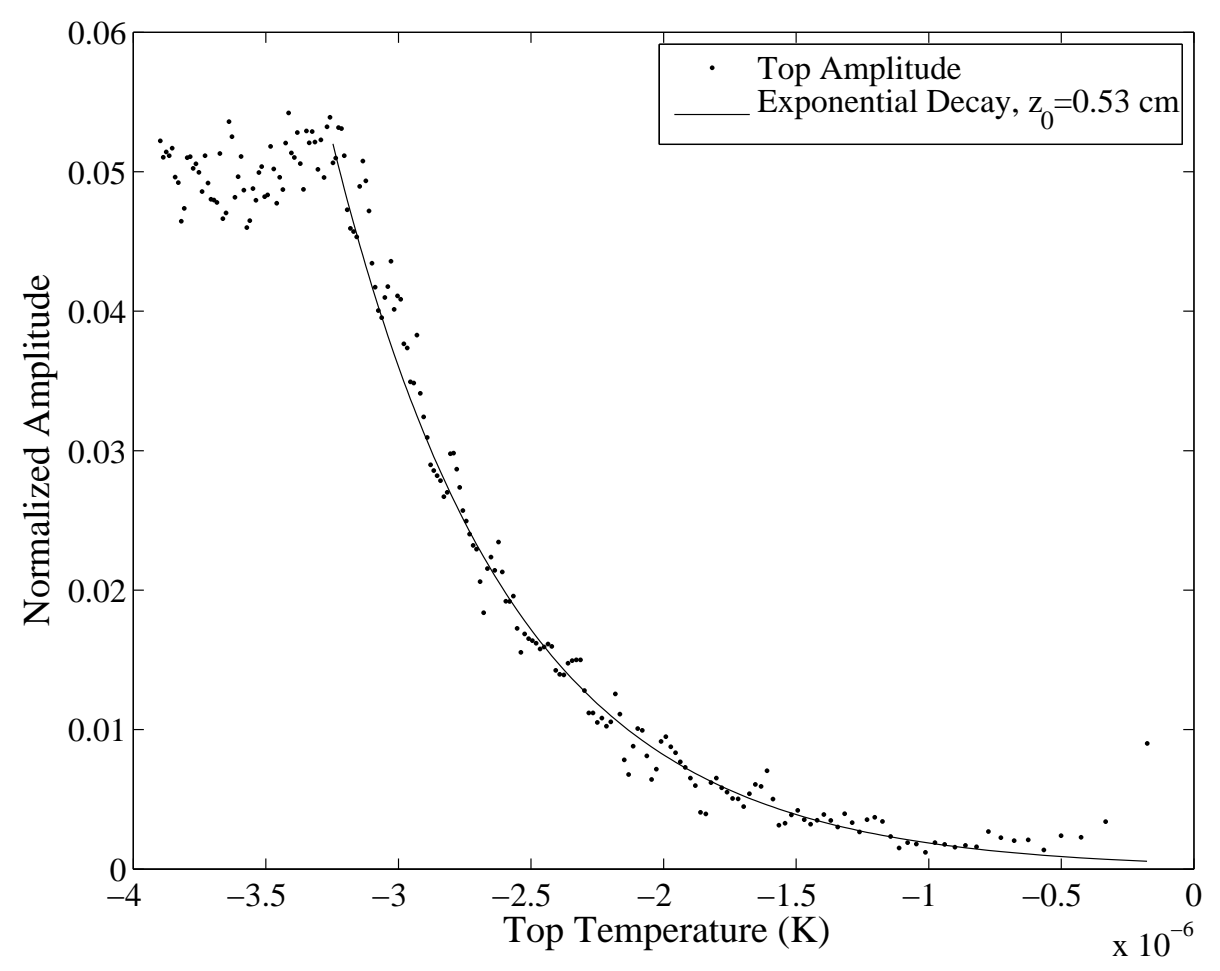

Figure 5.5. Attenuation of the SOC wave amplitude as measured by the top thermometer for $Q=61 \mathrm{nW} / \mathrm{cm}^{2}$. The amplitude is normalized as explained in the text.

\subsubsection{Attenuation}

As the wave travels upwards through the SOC state, its amplitude is attenuated as $\exp ^{-z / z_{0}}$, where $z$ is the distance traveled and $z_{0}$ is a parameter dependent both on $T_{\mathrm{SOC}}$ and the frequency of the excitation. In sec. 5.2.1, we have already computed the wave amplitude $A_{\mathrm{T}}$ as measured with $\mathrm{HRT}_{\mathrm{T}}$. $\mathrm{HRT}_{\mathrm{T}}$ measures the temperature of the layer of helium that lies above the SOC state. The response seen by the top thermometer is the integral of the heat transport from the SOC wave divided by the heat capacity of the helium above the SOC state at the top of the sample. Therefore, with a suitable measure of the heat capacity of this top layer, we can extract the attenuation of the SOC wave.

Fortunately, we have a continuously updated measure of the heat capacity of the helium above the SOC state. Since we are drifting up in temperature with a set amount of heat $\delta H$, the time rate of change in the top temperature is related to the heat capacity by, $\frac{d \mathrm{HRT}_{\mathrm{T}}}{d t}=\frac{\delta H}{C}$. As we are only concerned with the attenuation of the amplitude as opposed to a direct measure of the amplitude, it suffices to simply plot $A_{\mathrm{T}} / \frac{d \mathrm{HRT}_{\mathrm{T}}}{d t}$. An example of such a plot is given in fig. 5.5 along with an exponential fit. 


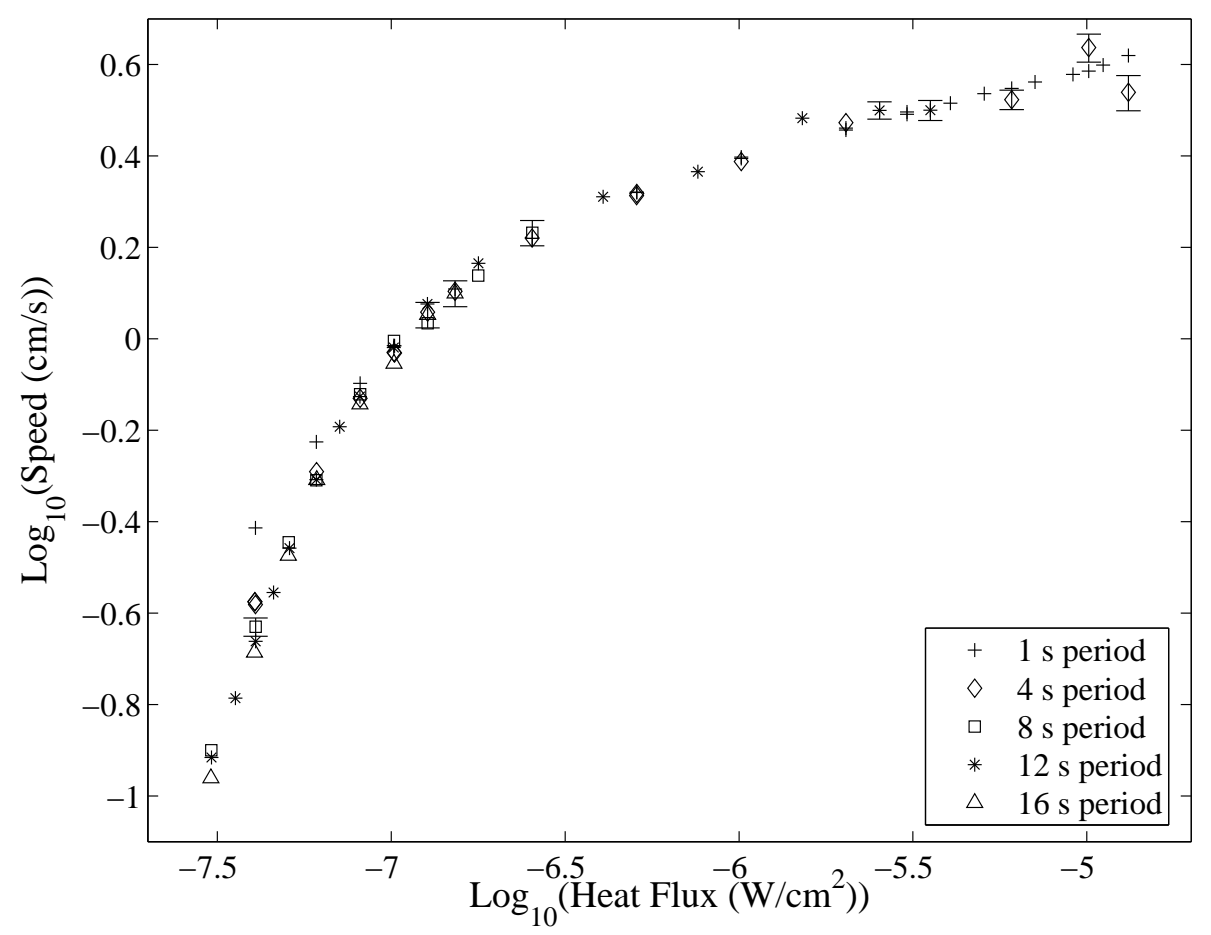

Figure 5.6. Wave speed vs. heat flux. Error bars are derived from the statistical error from the slope computation. Error bars smaller than the size of data point symbol, 4\%, are suppressed.

\subsection{Results and Conclusions}

\subsubsection{Speed}

The results for the speed of the SOC wave are presented in fig. 5.6. The first thing to note is that there is almost no frequency dependence on the speed of the wave. There are a few measurements at very low heat flux where we saw a frequency dependence in the speed (the two errant "+" symbols in particular). These points correspond to waves with very large attenuation, with $z_{0}$ less than one quarter of the sample height.

To compare to our prediction in eq. (1.15), we need values for the heat capacity and temperature versus heat flux. We extracted this information from the data from our SOC heat capacity runs. We fit $T_{\mathrm{SOC}}$ vs. $Q$ using the formula

$$
T_{\mathrm{SOC}}=T_{\lambda}-F\left(T_{\lambda}\left(Q / Q_{0}\right)^{0.813}\right)+T_{\lambda}\left(\kappa / \kappa_{0}\right)^{(-1 / x)}
$$

with values $x=0.8$ and $\kappa_{0}=20 \mathrm{nW} / \mathrm{cm} \mathrm{K}$. Originally, we modeled $T_{\mathrm{SOC}}$ using $F(X)=X$. However, as we saw in sec. 4.2.7, there is significant curvature in the fit residuals with a simple power law fit. We used a fourth-degree polynomial for $F$ to fit our results properly. ${ }^{3}$ A plot of the residuals

\footnotetext{
${ }^{3}$ A polynomial fit in $Q$ directly never gave a satisfactory fit. By using the variable $Q^{0.813}$, most of the dependence
} 


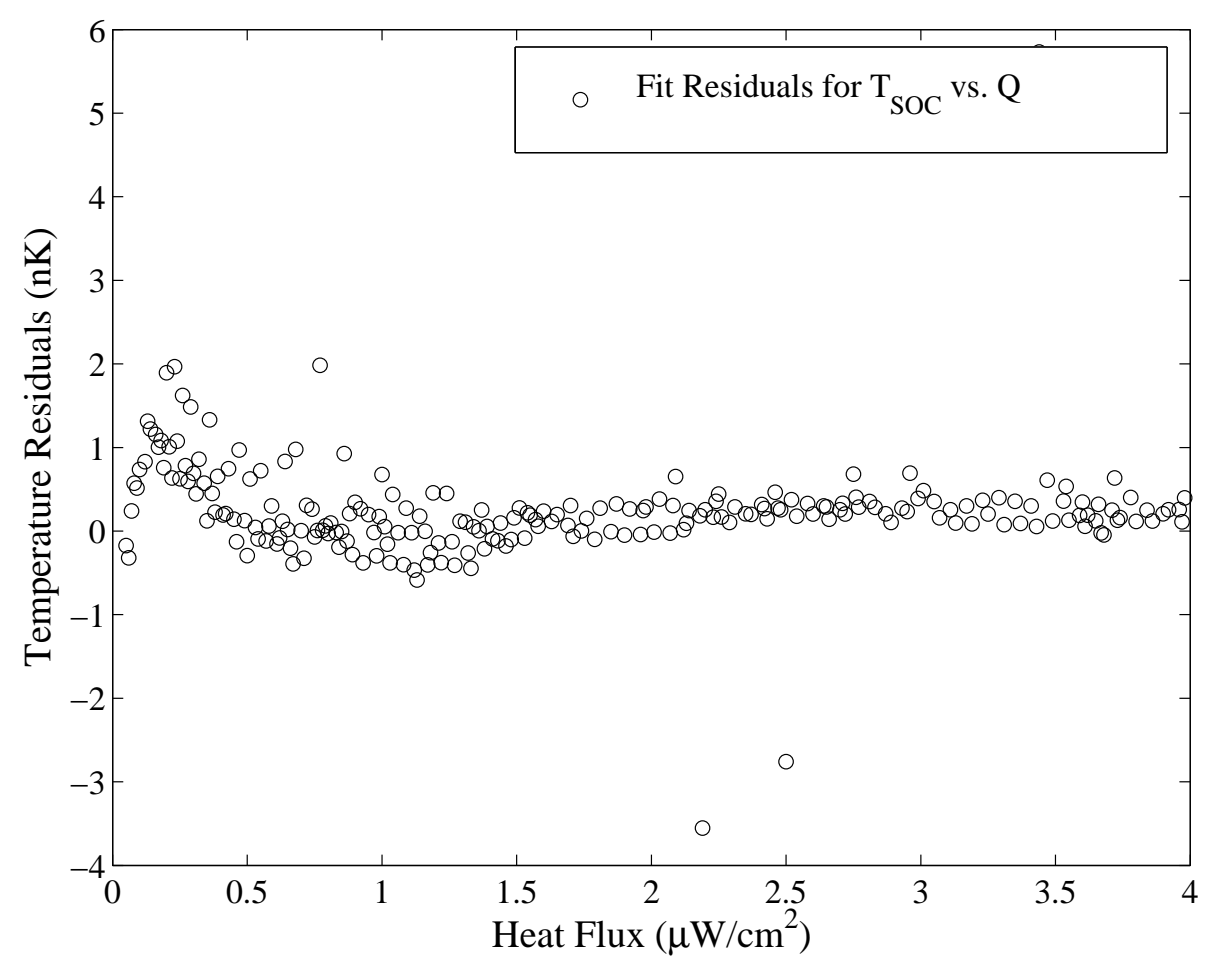

Figure 5.7. Residuals of the fit to the $T_{\mathrm{SOC}}$ vs. $Q$ results using eq. (5.6) and a fourth-degree polynomial for $F$.

of this fit is given in fig. 5.7. It is important to note that the residuals show that a single value of $\kappa_{0}$ and $x$ do not make a perfect fit. At low heat flux, the residuals grow briefly to $1 \mathrm{nK}$. Also, since the $T_{\mathrm{SOC}}$ vs. $Q$ data went to a minimum of $45 \mathrm{nW} / \mathrm{cm}^{2}$ and the $\mathrm{SOC}$ wave speed results used a minimum heat flux of $30 \mathrm{nW} / \mathrm{cm}^{2}$, we erred towards keeping the fit residuals above zero at the lowest $Q$. However, the fit is adequate for our purposes, keeping the computed temperature within a nK of $T_{\mathrm{SOC}}$. For the heat capacity, we divided our results into segments and fit each of them to fourth-degree polynomials.

We used the fits of the heat capacity and temperature along with eq. (1.15), $v=-\frac{1}{C} \frac{\partial Q_{\mathrm{SOC}}}{\partial T}$, to produce a prediction for the wave speed. In fig. 5.8, we see that this zero-adjustable-parameter prediction does an excellent job of reproducing our results.

\subsubsection{Attenuation}

The results for the attenuation of the SOC wave are presented in fig. 5.9. We plot $z_{0}$, the vertical distance over which the amplitude of the wave falls by $1 / e$, vs. the heat flux.

Recall from sec. 1.3 that we calculated the attenuation of the SOC wave. In order to make a was captured with a linear term, while the higher order terms served to capture the observed roll-off at high heat flux. 


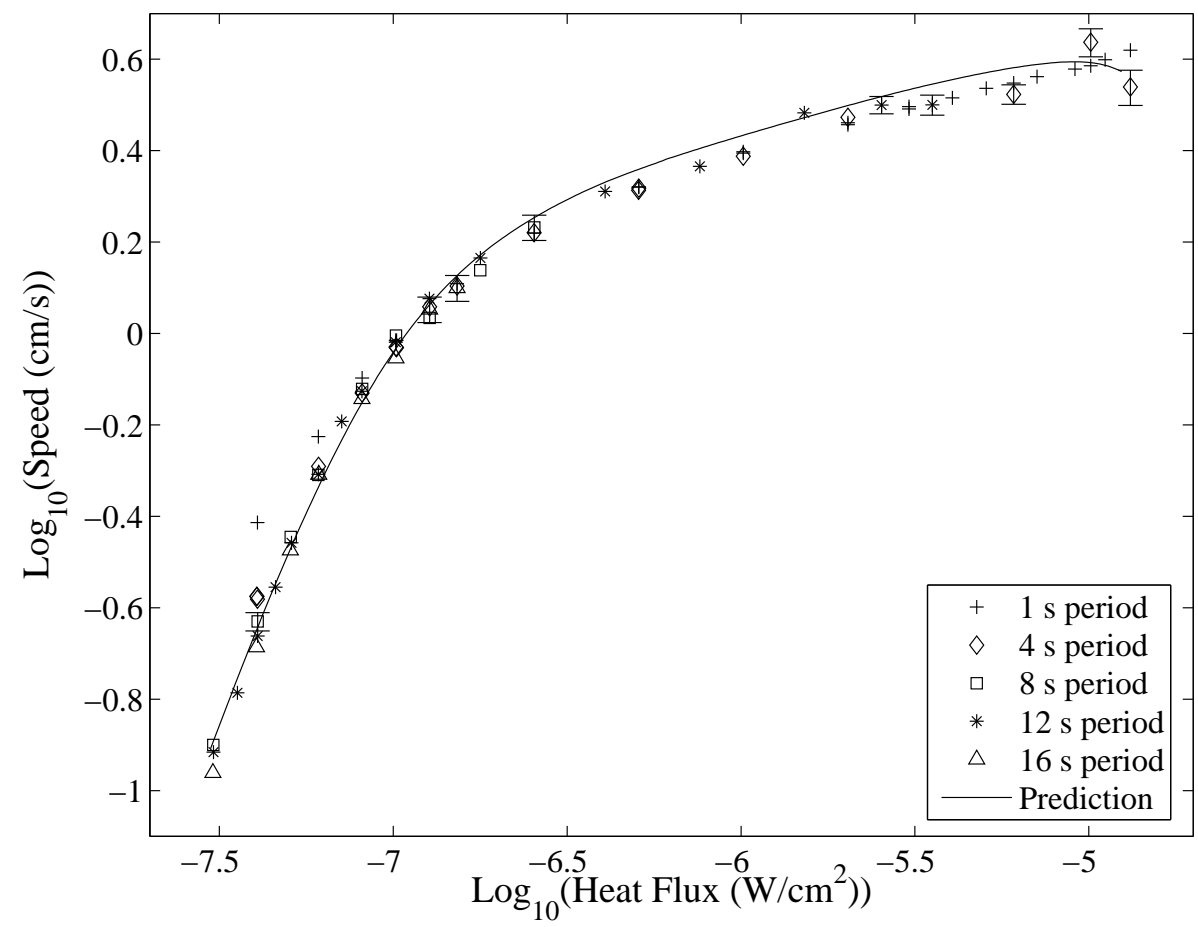

Figure 5.8. Wave speed vs. heat flux compared to the prediction of eq. (1.15). As discussed in the text, this prediction has no adjustable parameters; the values for both the heat capacity and $T_{\mathrm{SOC}}$ vs. $Q$ are from experimental our data. 


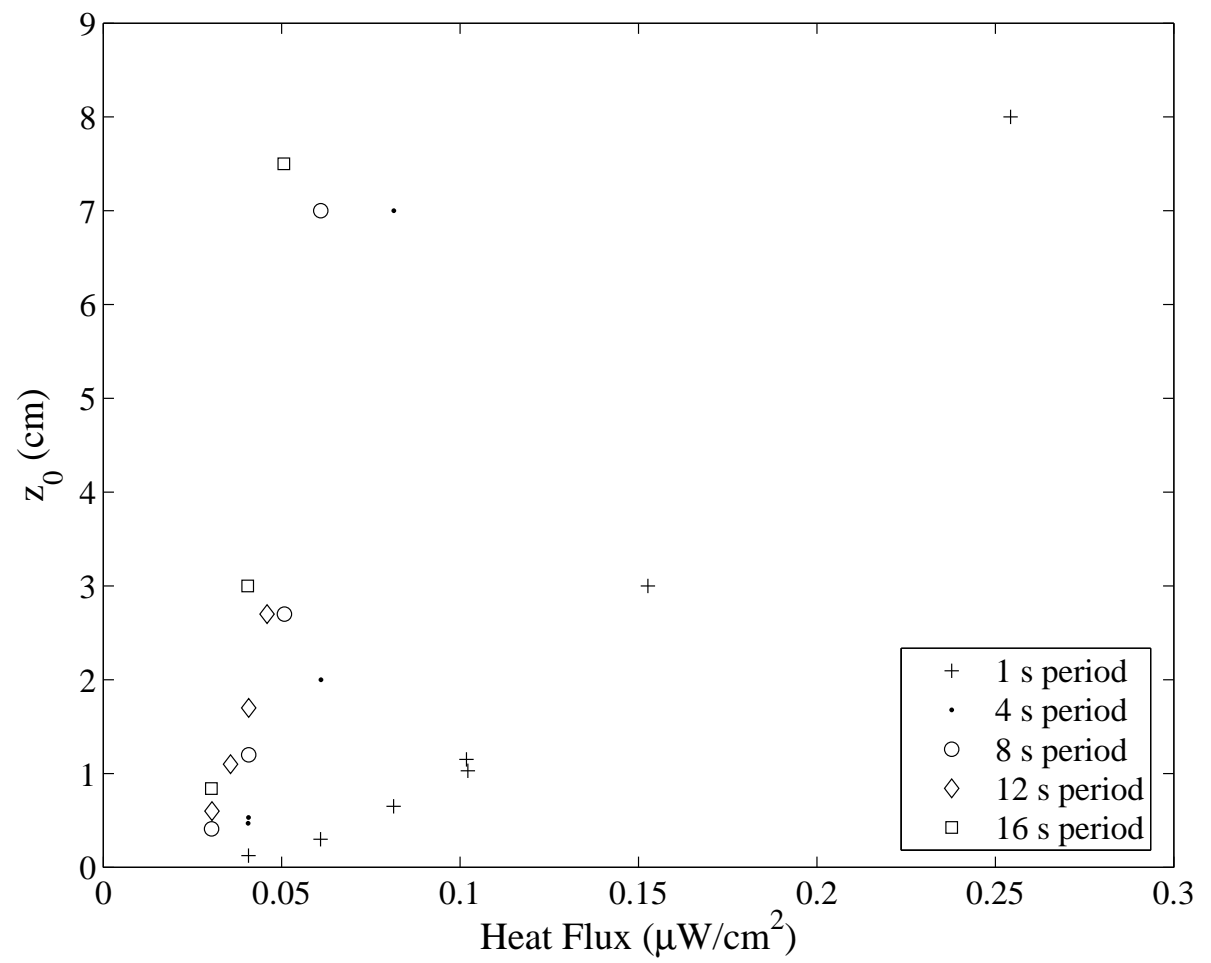

Figure 5.9. Attenuation plot showing $z_{0}$, the length over which the wave amplitude falls by $1 / e$ vs. the heat flux $Q$. 


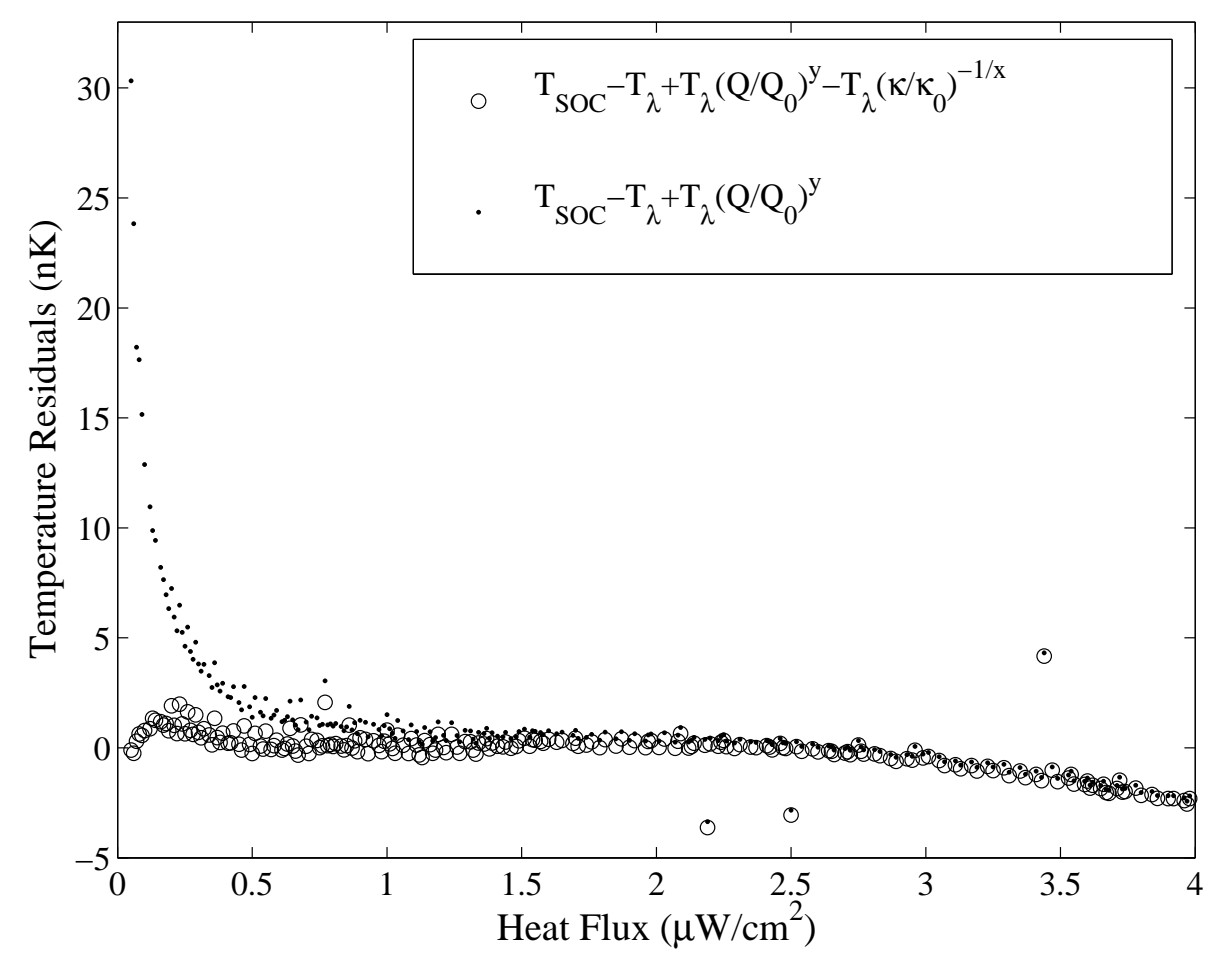

Figure 5.10. $T_{\mathrm{SOC}}$ vs. $Q$ fit residuals. The open circles are the residuals from the best fit to eq. (5.8). The best fit parameters are given in table 5.1. The dot symbols are the residuals using the same $Q_{0}$ from the first fit, but ignoring the $\kappa / \kappa_{0}$ term in the equation.

prediction (because we did not now how to measure $\frac{\partial \kappa}{\partial \epsilon^{\prime}}$ ) we modeled the thermal conductivity as

$$
\kappa=\kappa_{0} \theta^{-x}=\kappa_{0}\left(\frac{T-T_{\mathrm{C}}}{T_{\lambda}}\right)^{-x}
$$

where we use $T_{\mathrm{C}}=T_{\lambda}-T_{\lambda}\left(Q / Q_{0}\right)^{0.813}$. In order to make numerical predictions, we need to find the best values for the parameters $\kappa_{0}$ and $x$. We varied the parameters $Q_{0}, \kappa_{0}$, and $x$ in the equation ${ }^{4}$

$$
T_{\mathrm{SOC}}=T_{\lambda}-T_{\lambda}\left(Q / Q_{0}\right)^{0.813}+T_{\lambda}\left(\kappa / \kappa_{0}\right)^{(-1 / x)}
$$

to obtain a best fit to our $T_{\mathrm{SOC}}$ vs. $Q$ results. The residuals are shown in fig. 5.10 and the best fit parameters are given in table 5.1.

Table 5.1. Parameters for the fit of $T_{\mathrm{SOC}}$ vs. $Q$ using eq. (5.8)

$$
\begin{array}{lr}
\kappa_{0} & 20 \mathrm{nW} / \mathrm{cmK} \\
x & 0.8 \\
Q_{0} & 784 \mathrm{~W} / \mathrm{cm}^{2}
\end{array}
$$

\footnotetext{
${ }^{4}$ We use this simplified version because the attenuation results are limited to low heat fluxes where the added complexity of eq. (5.6) does not improve the fit.
} 
Fig. 5.10 also shows the residuals when ignoring the normal fluid thermal conductivity (the last term in eq. (5.8)) and modeling the SOC temperature as $T_{\mathrm{SOC}}=T_{\mathrm{C}}$. In sec. 4.2.7, we had assumed that $T_{\mathrm{SOC}}=T_{\mathrm{C}}$ for $Q>0.5 \mu \mathrm{W} / \mathrm{cm}^{2}$. These results suggest that is not true as there is a measurable difference up to at least $1 \mu \mathrm{W} / \mathrm{cm}^{2}$. However, we have kept our previous results for three reasons: the change in $Q_{0}$ is small, 784 vs. $767 \mu \mathrm{W} / \mathrm{cm}^{2}$; the change in $T_{\lambda}$ is small, $4 \mathrm{nK}$; and there is no independent confirmation that modeling the thermal conductivity using eq. (5.7) is the correct thing to do.

In sec. 1.3, the dissipation term was given as $e^{-D k^{2} t}$, however, experimentally, it was much easier to measure the attenuation in the form $e^{-z / z_{0}}$. These two terms should be equal, so

$$
D k^{2} t=z / z_{0}
$$

We can substitute in the value for $D$, given in eq. (1.24), $z=v t$, and $k=2 \pi f / v$ to get

$$
\frac{\kappa}{C}\left(1-\frac{1}{1+\frac{\theta}{x y}\left(\frac{Q}{Q_{0}}\right)^{-y}}\right) \frac{4 \pi^{2} f^{2} z_{0}}{v^{3}}=1 .
$$

Substituting $\theta=\left(\kappa / \kappa_{0}\right)^{(-1 / x)}$ and $\kappa=Q / \alpha$ gives

$$
\frac{Q}{\alpha C}\left(1-\frac{1}{1+\frac{1}{x y}\left(\frac{Q}{\alpha \kappa_{0}}\right)^{(-1 / x)}\left(\frac{Q}{Q_{0}}\right)^{-y}}\right) \frac{4 \pi^{2} f^{2} z_{0}}{v^{3}}=1 .
$$

Eq. (5.11) is a complicated formula, but all the parameters in it have all been computed or measured. If we plot the left-hand side and our measurements and our theory are correct, we should obtain 1. We plot this quantity vs. heat flux in fig. 5.11 and vs. $z_{0}$ in fig. 5.12. In each case, we expect the value 1 , but the results are slightly lower. This means that there is more dissipation than we calculated. However, we are mostly within a factor of 2 or 3 of the expected result. From the second figure, it appears there is some unaccounted for dependence on $z_{0}$. A likely source of error is the use of eq. (5.7) to model the thermal conductivity $\kappa$.

Previous attempts to calculate the dissipation of the SOC wave have ignored terms arising from the dependence of the thermal conductivity on the gradient in the temperature [2]. To illustrate how important this dependence is, we replot eq. (5.11), this time ignoring the effect of $\frac{\partial \kappa}{\partial \epsilon^{\prime}}$ by instead plotting

$$
\frac{Q}{\alpha C} \frac{4 \pi^{2} f^{2} z_{0}}{v^{3}}=1
$$

The results are shown in fig. 5.13. The formula significantly overestimates the dissipation for larger $Q$. However, the $\frac{\partial \kappa}{\partial \epsilon^{\prime}}$ term dropped from eq. (5.11) is negative and can only reduce the dissipation. 


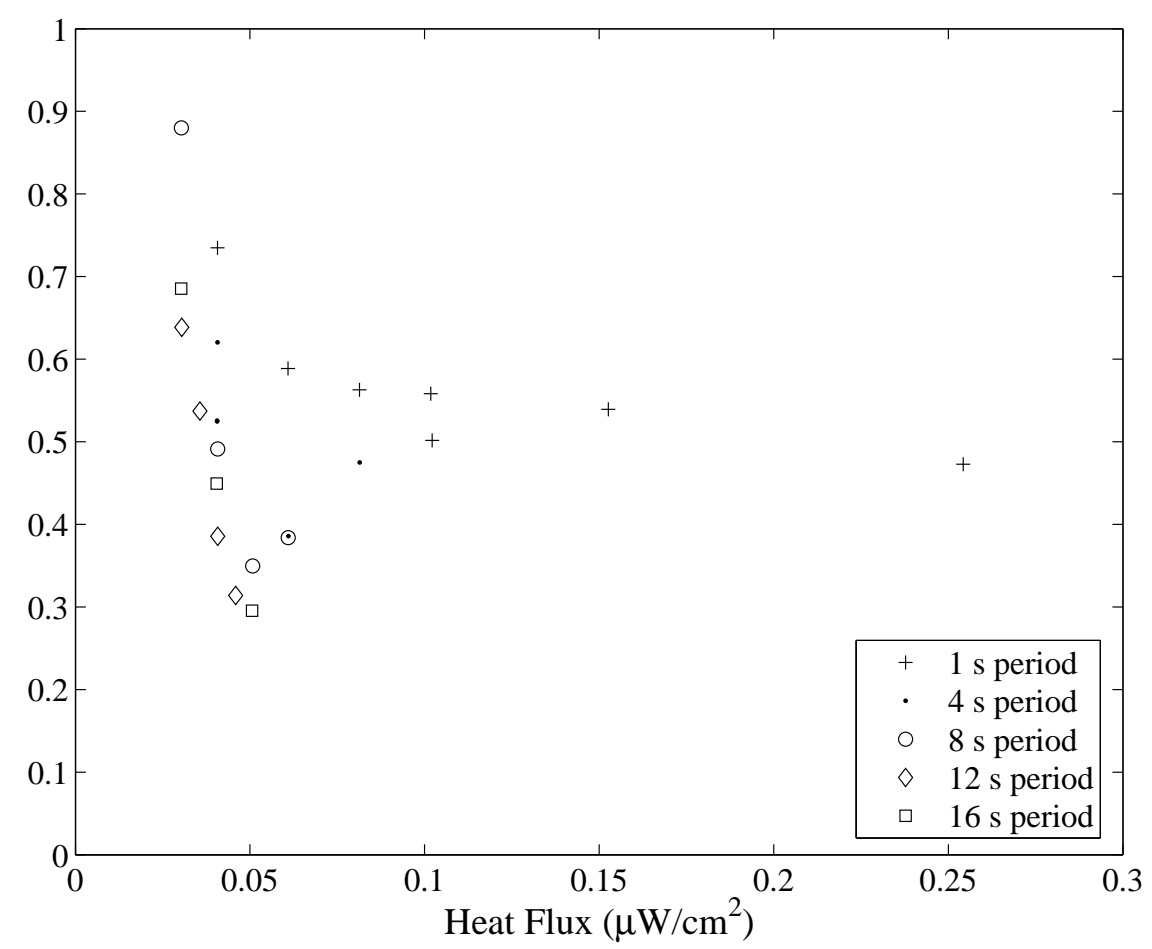

Figure 5.11. The attenuation length $z_{0}$ scaled to 1 by eq. (5.11) vs. the heat flux. If the dissipation is larger than expected, the results will be below 1, otherwise they will lie above.

Therefore, an imperfect model of $\kappa$ cannot be used to explain why more dissipation than expected is seen near $50 \mathrm{nW} / \mathrm{cm}^{2}$.

\subsection{Conclusion}

The simple model of treating the SOC state existing in the normal fluid with a depressed transition at $T_{\mathrm{C}}(Q)<T_{\lambda}$ worked surprisingly well in computing the speed and attenuation of the SOC wave. For the wave speed results in particular, the fit between theory and experiment is very good. This is probably because the results did not depend on a particular model for the conductivity. ${ }^{5}$ The comparison between theory and experiment is less successful for the attenuation results. However, the new prediction produced in this thesis (which included the response of the thermal conductivity to a change in temperature gradient through the second term in eq. (1.12)) correctly predicts a significant drop in the attenuation for larger heat flux.

\footnotetext{
${ }^{5}$ Although we did appear to use the model to fit our data in sec. 5.3.1, we did not need to extrapolate properties beyond what we experimentally verified. In the attenuation results, however, we used the same fit to predict the thermal conductivity for different temperature gradients, which we did not experimentally verify.
} 


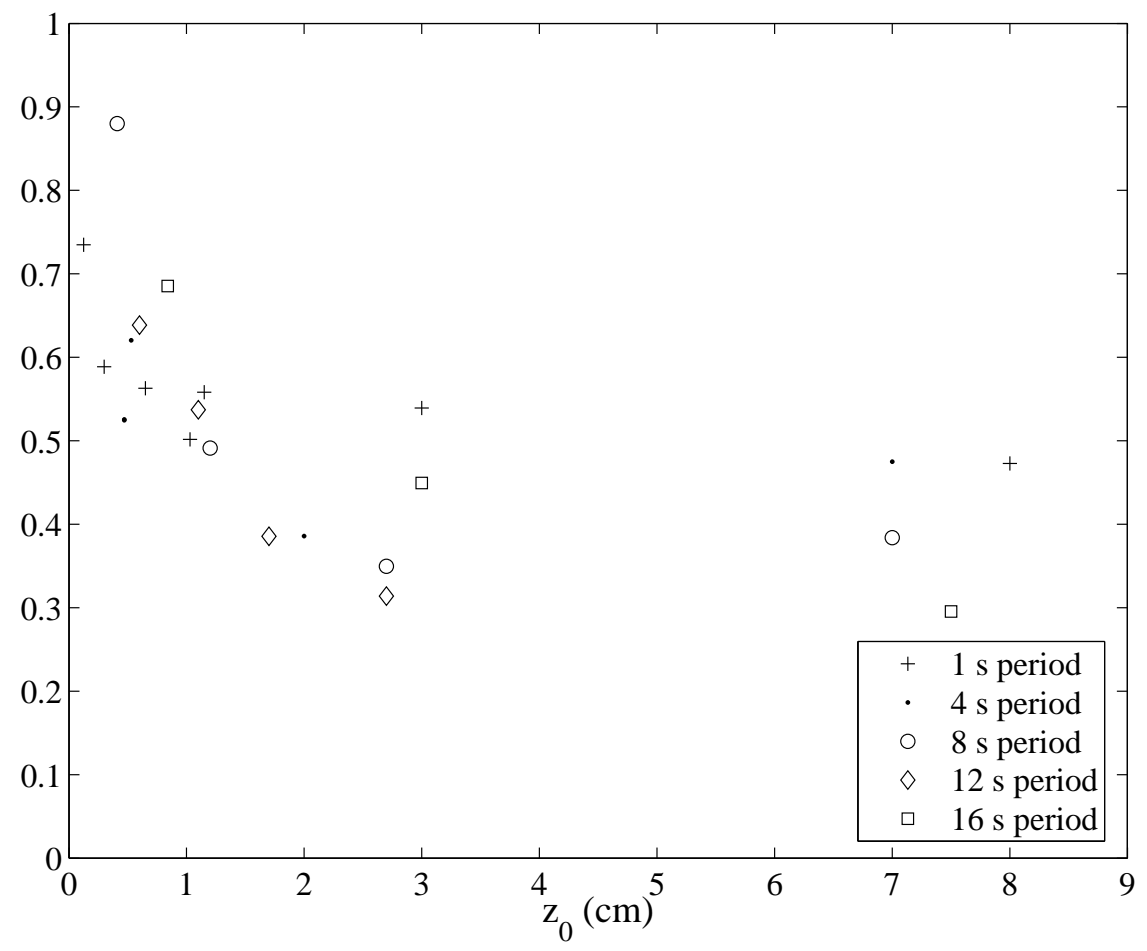

Figure 5.12. The attenuation length $z_{0}$ scaled to 1 by eq. (5.11) vs. the attenuation length. If the dissipation is larger than expected, the results will be below 1 , otherwise they will lie above. 


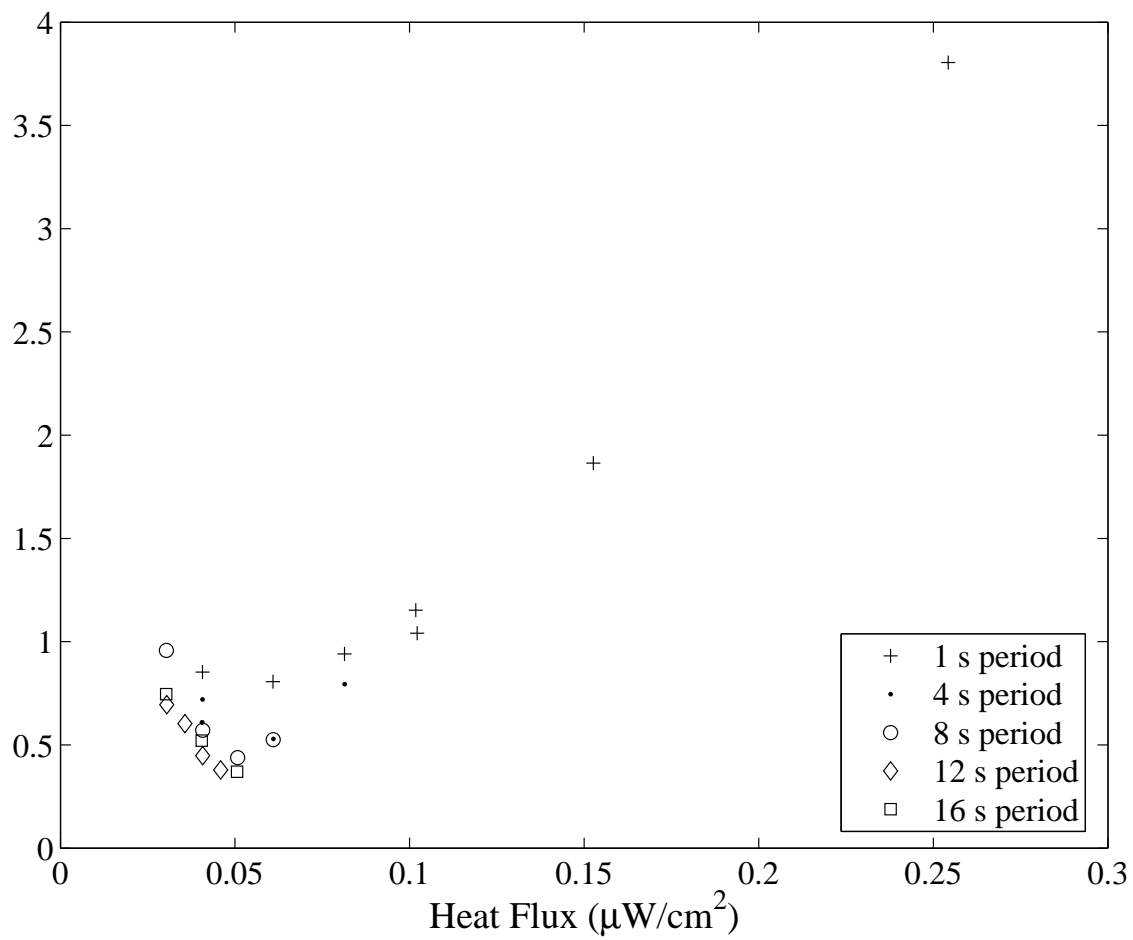

Figure 5.13. The attenuation length $z_{0}$ scaled to 1 by eq. (5.12) vs. the heat flux. If the dissipation is larger than expected, the results will be below 1, otherwise they will lie above. For high heat flux, the dissipation is much lower than predicted by the simplified model. 


\section{Chapter 6}

\section{Summary}

\section{$6.1 \quad T_{\text {SOC }}$ vs. $Q$}

We repeated measurements of $T_{\mathrm{SOC}}$ vs. $Q$ and found for heat fluxes $Q<5 \mu \mathrm{W} / \mathrm{cm}^{2}$ that our results were reasonably consistent with $t_{\mathrm{SOC}}(Q)=\left(Q / Q_{0}\right)^{0.813}$, with $Q_{0}=767 \pm 20 \mu \mathrm{W} / \mathrm{cm}^{2}$. These results are shown in fig. 6.1. Our result is consistent ${ }^{1}$ with $Q_{0}=745 \mu \mathrm{W} / \mathrm{cm}^{2}$ from Lee et al. [3] but disagrees $^{2}$ with the original result from Moeur et al. of $Q_{0}=638 \mu \mathrm{W} / \mathrm{cm}^{2}$ [1]. However, it is important to stress that, as seen in fig. 6.2, there is significant curvature in the region of the fit and there are no parameter values that convincingly satisfy $t_{\mathrm{SOC}}=\left(Q / Q_{0}\right)^{y}$ even over this reduced range of heat flux. For our higher heat fluxes $5 \mu \mathrm{W} / \mathrm{cm}^{2}<Q<13 \mu \mathrm{W} / \mathrm{cm}^{2}$ there is a much larger departure from the power law behavior. It is possible that at these larger heat flux values the helium is self-organizing as a result of the resistance caused by mutual friction as opposed to a staircase mechanism proposed by Weichman and Miller [7].

\subsection{SOC Heat Capacity}

We measured the SOC state heat capacity, $C_{\nabla T}$, over the range of heat fluxes $60 \mathrm{nW} / \mathrm{cm}^{2}<Q<$ $13 \mu \mathrm{W} / \mathrm{cm}^{2}$. The results are plotted in fig. 6.3 and show small departures of $C_{\nabla T}$ from the zerogravity static heat capacity, $C_{0}$. The similarities between the SOC heat capacity and $C_{0}$ suggests that this SOC state, at least for $T_{\mathrm{SOC}}<50 \mathrm{nK}$ below $T_{\lambda}$, is essentially in the superfluid phase. As suggested in the superfluid staircase model described in sec. 1.1, there needs to be periodic injections of normal fluid in order to maintain the temperature gradient. However, the near divergent heat

\footnotetext{
${ }^{1}$ There is some confusion as to the reporting of errors with respect to $Q_{0}$. Allowing both $Q_{0}$ and the exponent (which I have fixed at 0.813 ) to vary gives very large errors on each of the parameters because the effect of one parameter can be partially offset with a change the other. For example Moeur et al. reported $Q_{0}=638 \pm 178 \mathrm{~W} / \mathrm{cm}^{2}$ and $x=0.813 \pm 0.012$ [1]. Since our result falls within their error bar for $Q_{0}$, one might be tempted to say that they agree. However, fixing the exponent would surely result in a smaller error bar for $Q_{0}$. At $6 \mu \mathrm{W} / \mathrm{cm}^{2}$, the maximum heat flux for the Moeur et al. results, using $Q_{0}=767 \mathrm{~W} / \mathrm{cm}^{2}$ instead of $Q_{0}=638 \mathrm{~W} / \mathrm{cm}^{2}$ results in a $90 \mathrm{nK}$ difference in the computed $T_{\mathrm{SOC}}$. This discrepancy is well within the experimental resolution of both experiments.

${ }^{2}$ It is now believed that the discrepancy could have caused by early endplate designs which did not ensure an even distribution of heat into the helium.
} 


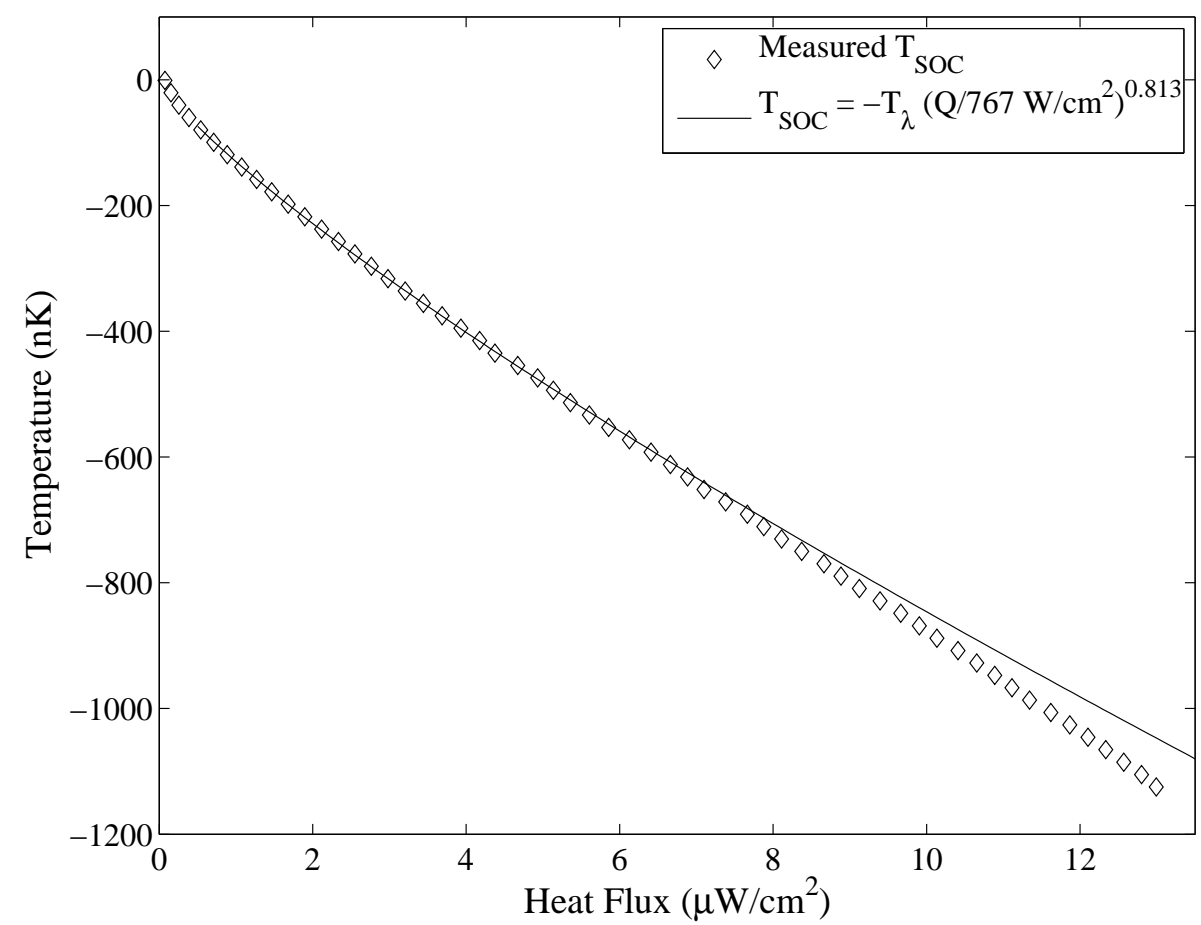

Figure 6.1. $T_{\mathrm{SOC}}$ vs. $Q$. For $0.5 \mu \mathrm{W} / \mathrm{cm}^{2}<Q<5 \mu \mathrm{W} / \mathrm{cm}^{2}, t_{\mathrm{SOC}(\mathrm{Q})}=\frac{T_{\lambda}-T_{\mathrm{SOC}}(Q)}{T_{\lambda}} \simeq$ $\left(Q / 767 \mathrm{~W} / \mathrm{cm}^{2}\right)^{0.813}$.

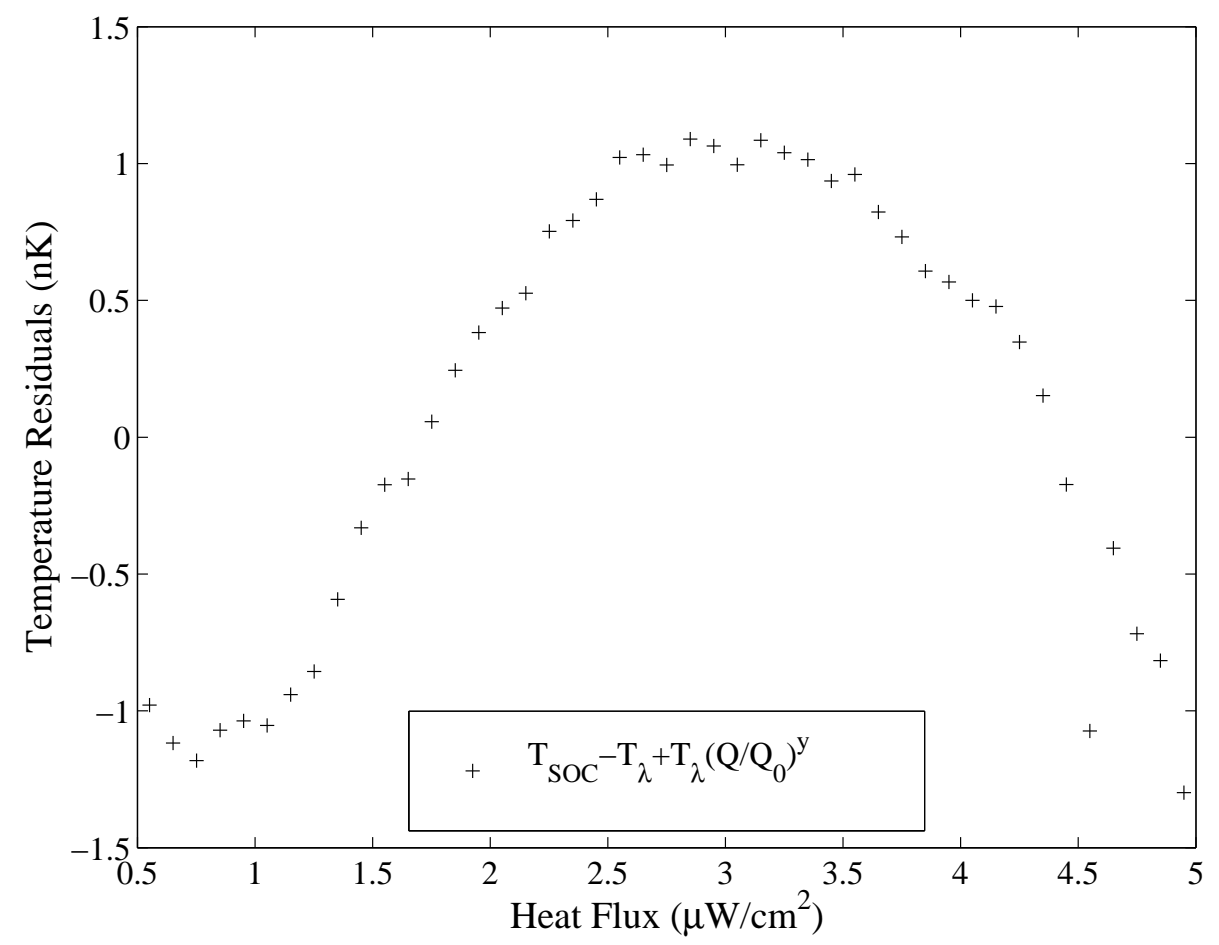

Figure 6.2. $T_{\mathrm{SOC}}$ vs. $Q$ fit residuals. The fit parameters are $Q_{0}=767 \mathrm{~W} / \mathrm{cm}^{2}$ and $x=0.813$. 


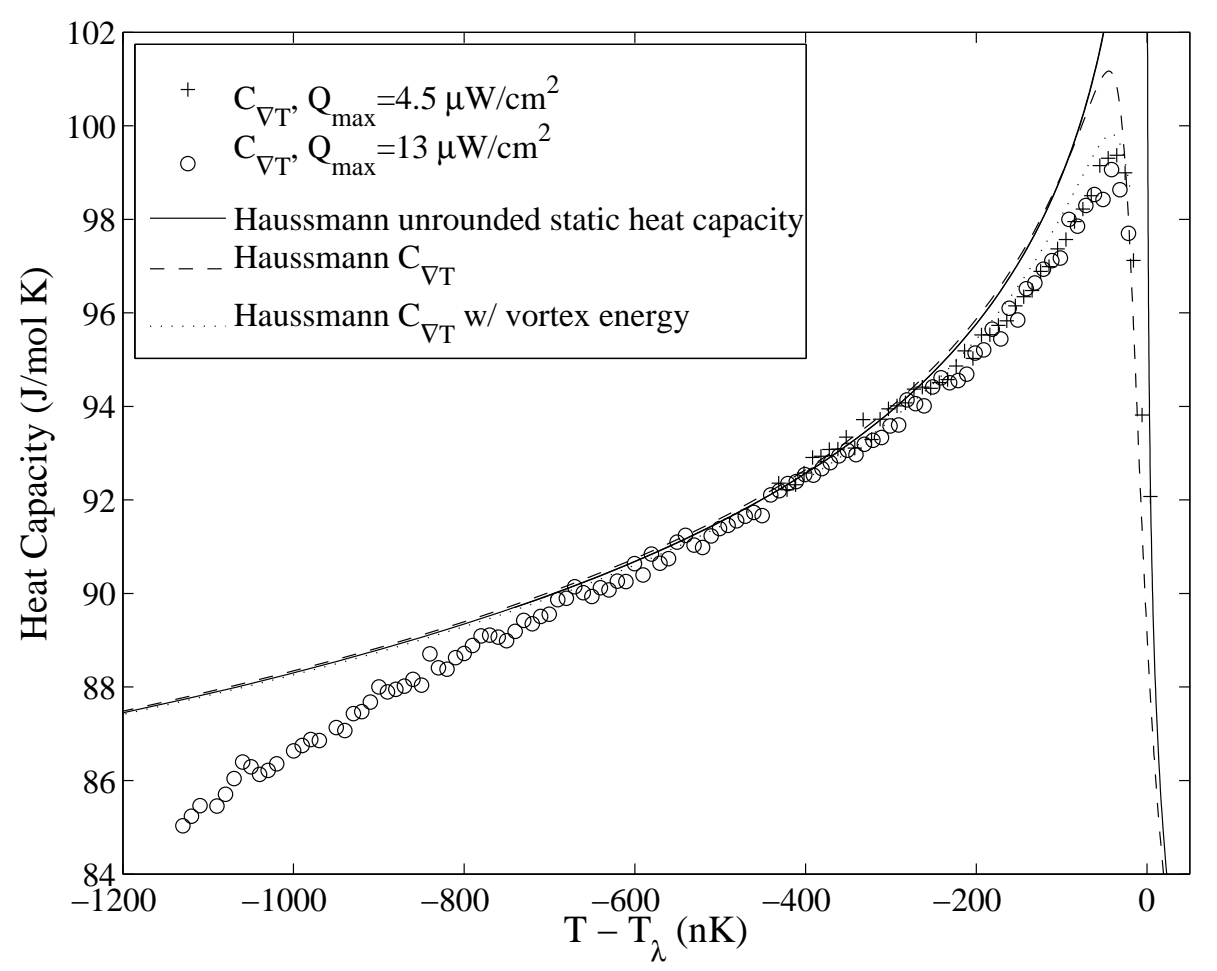

Figure 6.3. SOC heat capacity, $C_{\nabla T}$.

capacity of $C_{0}$ is due to the increasing size and number of fluctuations between the two phases helium: superfluid (He-II) and the normal fluid (He-I). These fluctuations occur on all length scales less than the correlation length, $\xi$, which diverges at $T_{\lambda}$. For $C_{\nabla T}$ to be the same as $C_{0}$, the majority of the helium must be in superfluid regions that are larger than the correlation length, the maximum size of fluctuations.

Within $200 \mathrm{nK}$ of $T_{\lambda}, C_{\nabla T}$ is depressed relative to $C_{0}$ and reaches its maximum at $50 \mathrm{nK}$ below $T_{\lambda}$. This depression in the heat capacity, while larger than either of the DRG predictions of Haussmann, appear very similar to the results that include the vortex core energy. Alternatively, using the one-dimensional staircase model, one can imagine a significant suppression of fluctuations when the correlation length approaches the size of the rise portion of each step.

At high heat flux, $Q>6 \mu \mathrm{W} / \mathrm{cm}^{2}$ and $T_{\mathrm{SOC}}<600 \mathrm{nW} / \mathrm{cm}^{2}, C_{\nabla T}$ departs downwards again from the static $C_{0}$. This is the same region where we saw a significant increase in the resistance due to mutual friction and a substantial departure from the fit for $t_{\mathrm{SOC}}$. Perhaps the proliferation of vortices and the interactions between them is suppressing the fluctuations and reducing the heat capacity. However, the DRG calculations of Haussmann predict no such departure from the static heat capacity at high heat flux. 


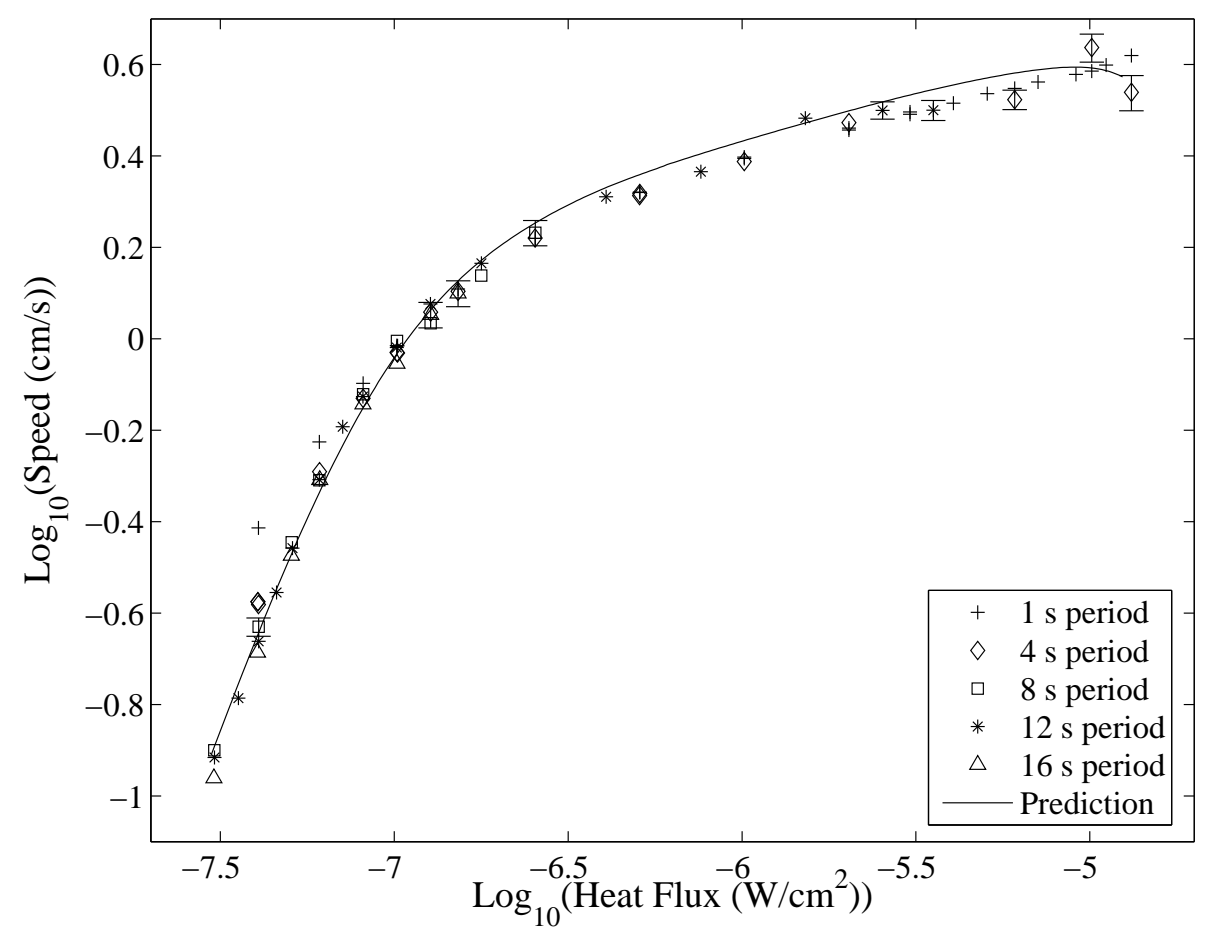

Figure 6.4. Wave speed vs. heat flux compared to the prediction from eq. (1.15).

\subsection{SOC Wave}

In order to understand a wave that only travels upwards through the SOC state, we treated the SOC helium as a fluid with a thermal conductivity that was a function of both the reduced temperature, $\epsilon$, and the gradient in reduced temperature, $\epsilon^{\prime}$. We computed the speed, $v=-\frac{\alpha}{C T_{\lambda}} \frac{\partial \kappa}{\partial \epsilon}$, and attenuation, $D=\frac{\kappa}{C}+\frac{\alpha}{C T_{\lambda}} \frac{\partial \kappa}{\partial \epsilon^{\prime}}$. The experimental results for the speed fit the prediction very well, as shown in fig. 6.4. However, to compute the attenuation, it was necessary to model the thermal conductivity as in eq. (1.1) and the prediction was not as close to our experimental results. In fig. 6.5, we plot the scaled attenuation length, $z_{0} / z_{0}$,Theory. The attenuation length is the distance over which the amplitude of the wave falls by $1 / e$. If our calculations are correct the scaled attenuation should always be 1 . The results are systematically low (therefore, we see more dissipation than expected) although they are not off by much more than a factor of 2 .

The success of modeling the SOC wave as a normal fluid seems to contradict our results for the SOC heat capacity, which suggested that the SOC state is best viewed as existing in the superfluid. However, each of these outlooks depends on different length scales. For the SOC wave, we are only concerned with how the fluid behaves on lengths similar to the wave length (which are close to $1 \mathrm{~cm})$. Viewed at these length scales, we integrate over whatever mechanism maintains the gradient in order to find the macroscopic transport properties. For the SOC heat capacity, however, we are 


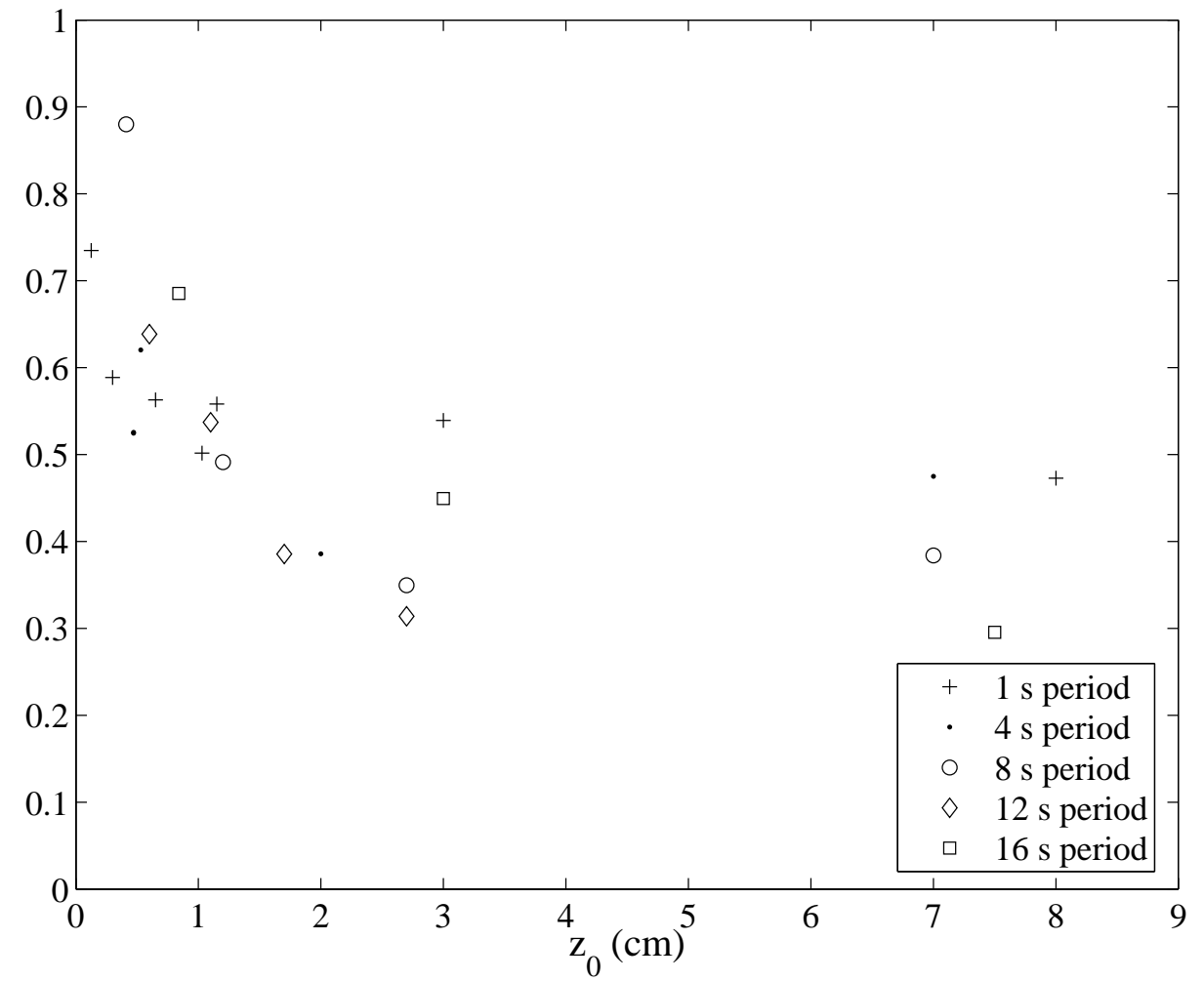

Figure 6.5. The attenuation length $z_{0}$ scaled to 1 by eq. (5.11) vs. the attenuation length. If the dissipation is larger than expected, the value will be below 1 , otherwise it will be above. 
concerned about the nature of the fluid on length scales similar to the correlation length which is less than $20 \mu \mathrm{m}$ for temperatures more than $50 \mathrm{nK}$ below $T_{\lambda}$. Therefore, in our opinion, there is no contradiction and the SOC state can behave both like a superfluid and a normal fluid. 


\section{Bibliography}

[1] W. A. Moeur, P. K. Day, F-C. Liu, S. T. P. Boyd, M. J. Adrianns, and R. V. Duncan. Phys. Rev. Lett., 78(12):2421, 1997.

[2] D. A. Sergatskov, A. V. Babkin, S. T. P. Boyd, R. A. M. Lee, and R. V. Duncan. J. Low Temp. Phys., 134(1-2):517, 2004.

[3] R. A. M. Lee, A. R. Chatto, D. A. Sergatskov, A. V. Babkin, S. T. P. Boyd, A. M. Churilov, T. D. McCarson, T. C. P. Chui, P. K. Day, R. V. Duncan, and D. L. Goodstein. J. Low Temp. Phys., 134(1-2):495, 2004.

[4] W. Y. Tam and G. Ahlers. Phys. Rev. B, 32(9):5932, 1985.

[5] A. Onuki. Jpns. J. Appl. Phys., 26-S3:365, 1987.

[6] P. K. Day, W. A. Moeur, S. S. McCready, D. A. Sergatskov, F-C. Liu, and R. V. Duncan. Phys. Rev. Lett., 81(12):2474, 1998.

[7] P. B. Weichman and J. Miller. J. Low Temp. Phys., 119(1-2):155, 2000.

[8] D. L. Goodstein. States of Matter. Dover, New York, 1985.

[9] R. Haussmann. Phys. Rev. B, 60(17):12349, 1999.

[10] T. C. P. Chui, D. L. Goodstein, and A. W. Harter. Phys. Rev. Lett., 77(9):1793, 1996.

[11] A. W. Harter, R. A. M. Lee, A. Chatto, X. Wu, T. C. P. Chui, and D. L. Goodstein. Phys. Rev. Lett., 84:2195, 2000.

[12] P. Day, I. Hahn, T. C. P. Chui, A. W. Harter, D. Rowe, and J. A. Lipa. J. Low Temp. Phys., 107(3-4):359, 1997.

[13] P. Welander, M. Barmatz, and I. Hahn. IEEE Trans. Instrum. Meas., 49(2):253, 2000.

[14] D. Murphy and H. Meyer. J. Low Temp. Phys., 105(1-2):185, 1996.

[15] R. V. Duncan and G. Ahlers. Phys. Rev. B, 43(10):7707, 1991. 
[16] M. van de Voorde. IEEE Transactions on Nuclear Science, NS-20(3):693, 1973.

[17] E. Kerr and R. Taylor. Annals of Physics, 26:292, 1964.

[18] J. C. Findlay, A. Pitt, H. Grayson Smith, and J. O. Wilhelm. Phys. Rev., 54:506, 1938.

[19] R. Donnelly and C. Barenghi. J. Phys. Chem. Ref. Data, 27(6):1217, 1998.

[20] J. A. Nissen, D. R. Swanson, X. Qin, and J. A. Lipa. Czech. J. Phys., 46(S1):379, 1996.

[21] K. Kuehn, S. Mehta, H. Fu, E. Genio, D. Murphy, F-C. Liu, Y. Liu, and G. Ahlers. Phys. Rev. Lett., 88(9):095702, 2002.

[22] J. A. Lipa, J. A. Nissen, D. A. Stricker, D. R. Swanson, and Chui T. C. P. Phys. Rev. B, 68(17):174518, 2003.

[23] H. Baddar, G. Ahlers, K. Kuehn, and H. Fu. J. Low Temp. Phys., 119:1, 2000.

[24] R. Haussmann, 2005. private communication.

[25] S. T. P. Boyd, D. Sergatskov, and R. V. Duncan. To be published in the proceedings for LT24.

[26] L. S. Goldner and G. Ahlers. Phys. Rev. B, 45(22):13129, 1992.

[27] L. S. Goldner, G. Ahlers, and R. Mehrotra. Phys. Rev. B, 43(16):12861, 1991. 
Appendix A

Cell Drawings 


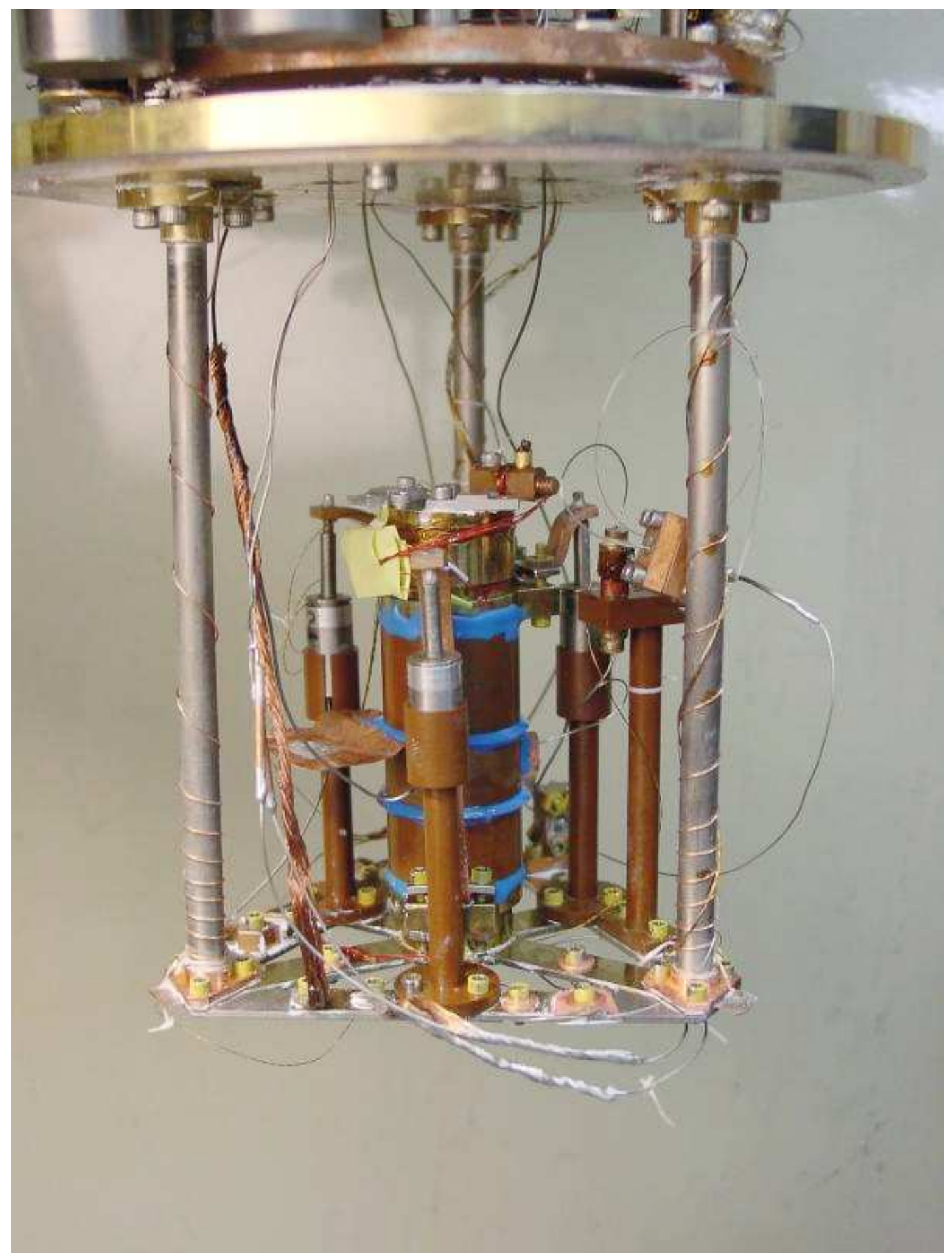

Figure A.1. A photo of the cell stage. 

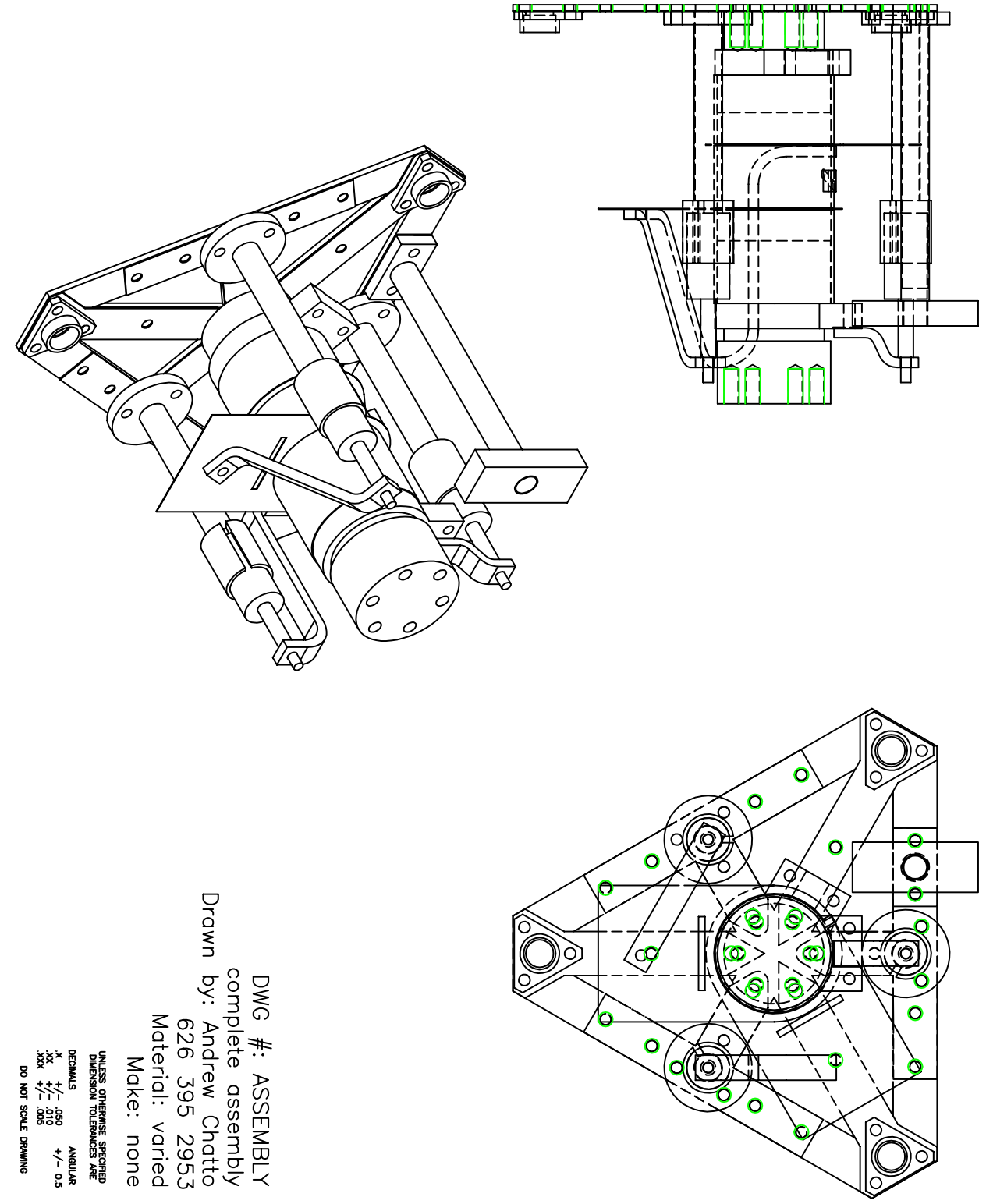

Figure A.2. A CAD rendering of the cell stage. 

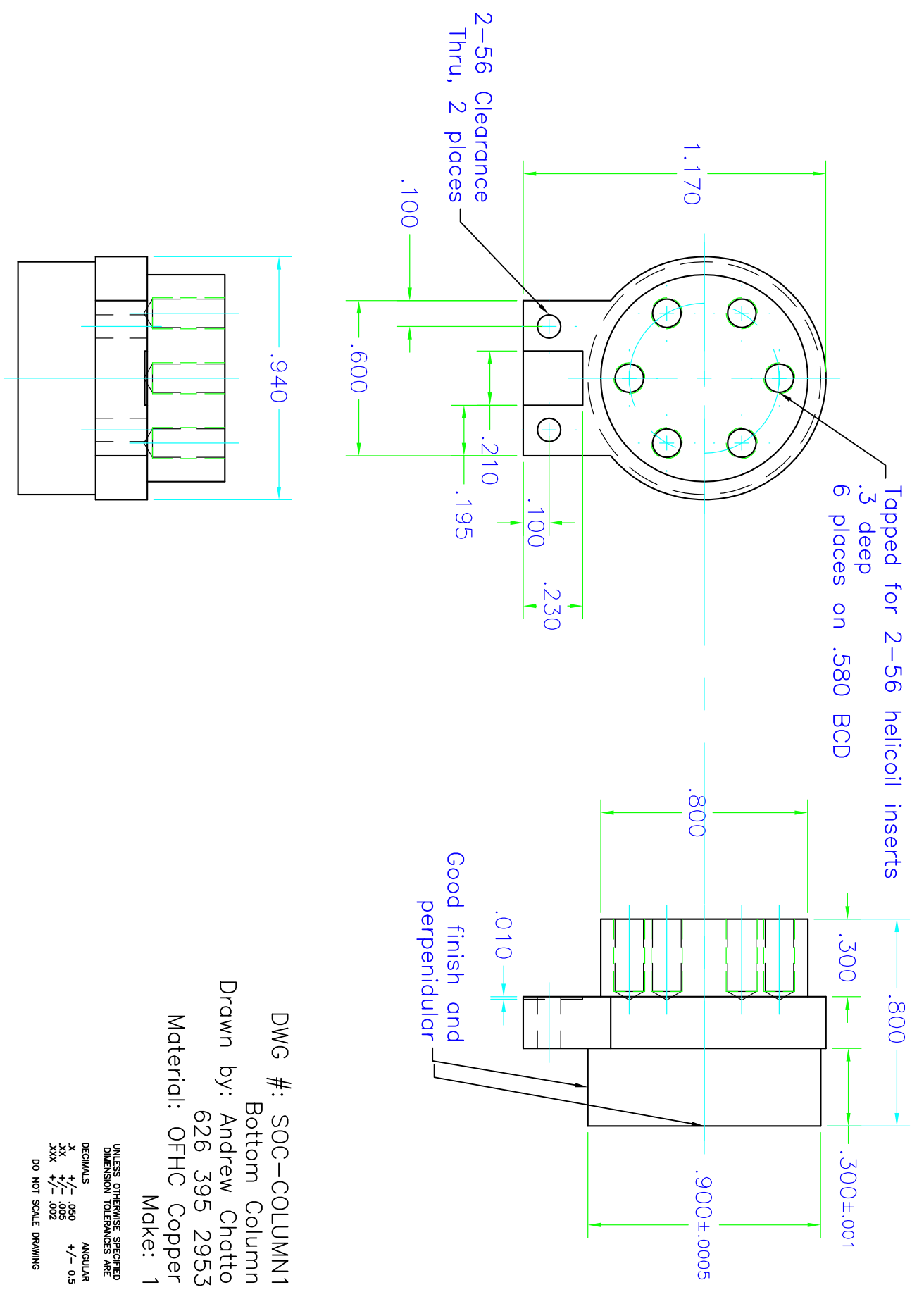

Figure A.3. The bottom endplate. 


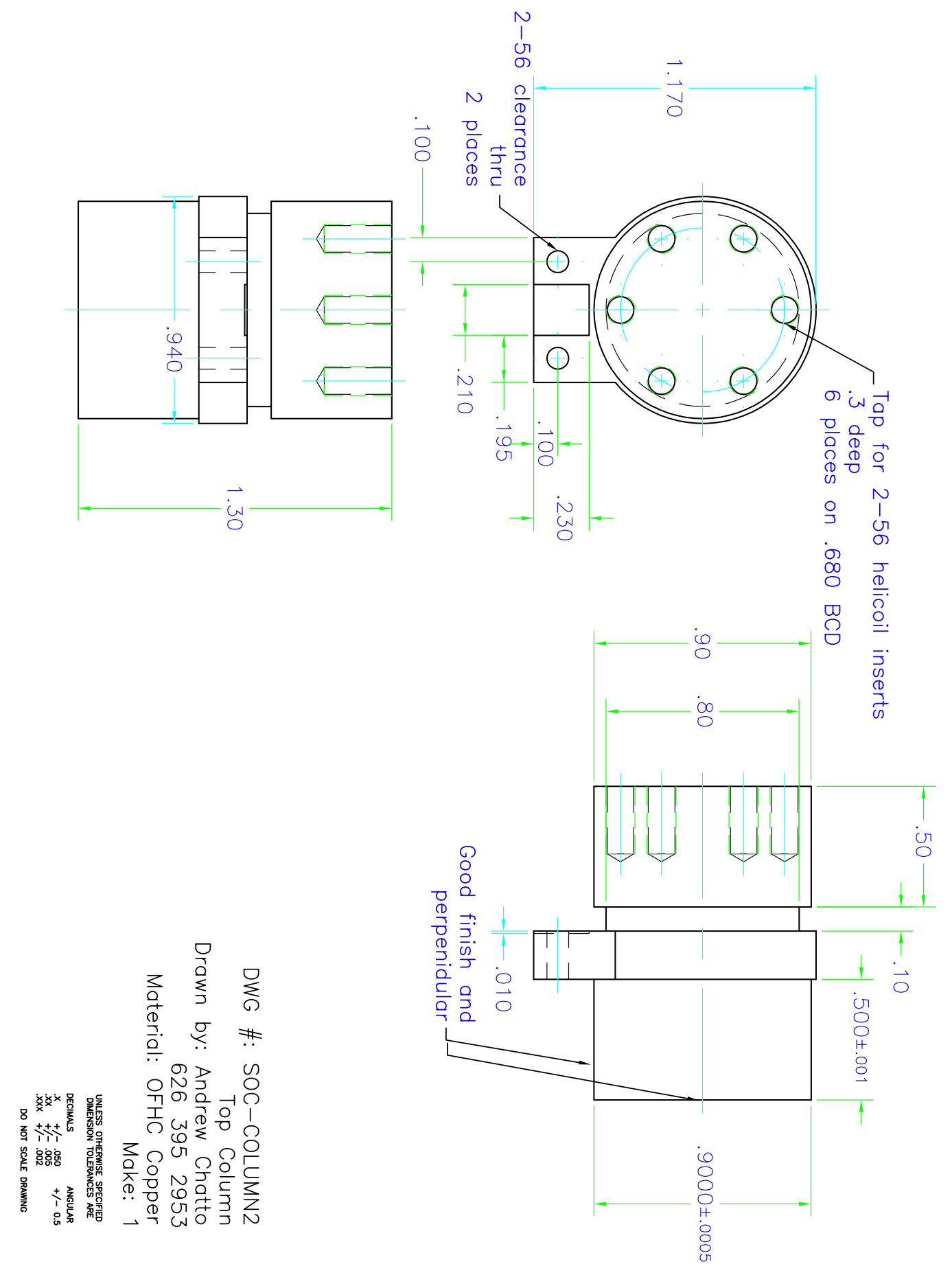

Figure A.4. The top endplate. 

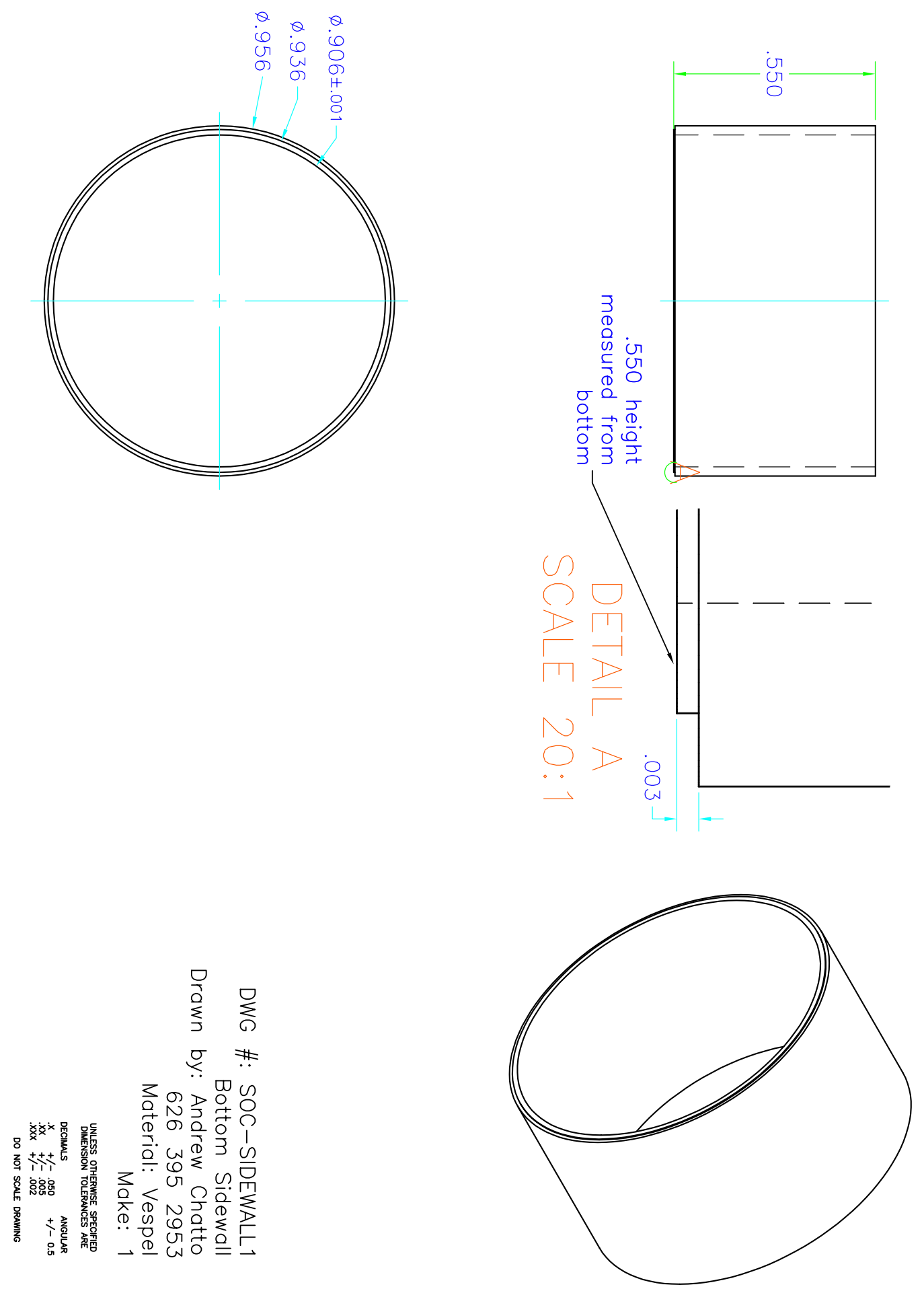

Figure A.5. The lowest piece of the Vespel sidewall. 

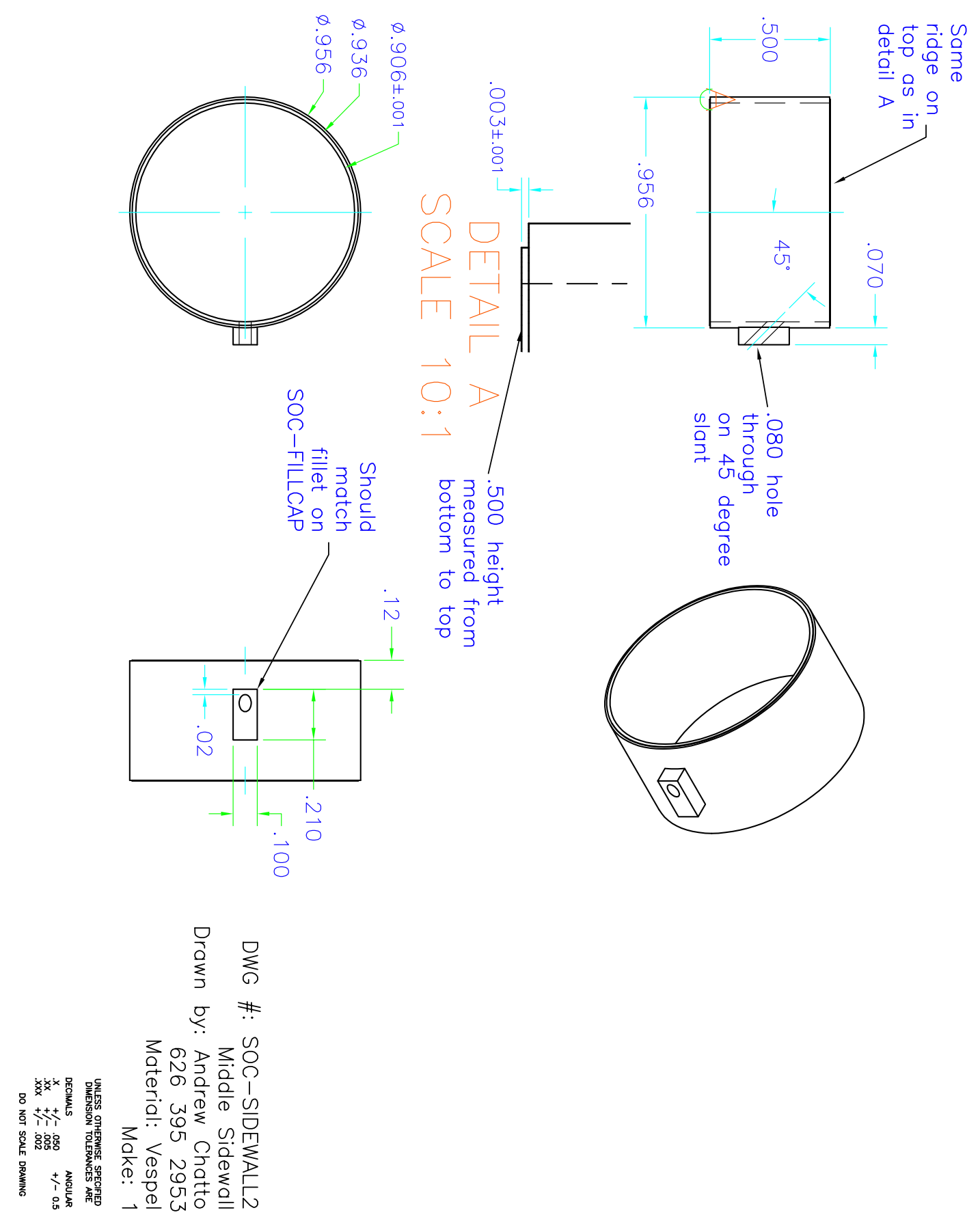

Figure A.6. The middle piece of the Vespel sidewall. The hole in the side is for the fill line and is pointed upwards as it exits. 

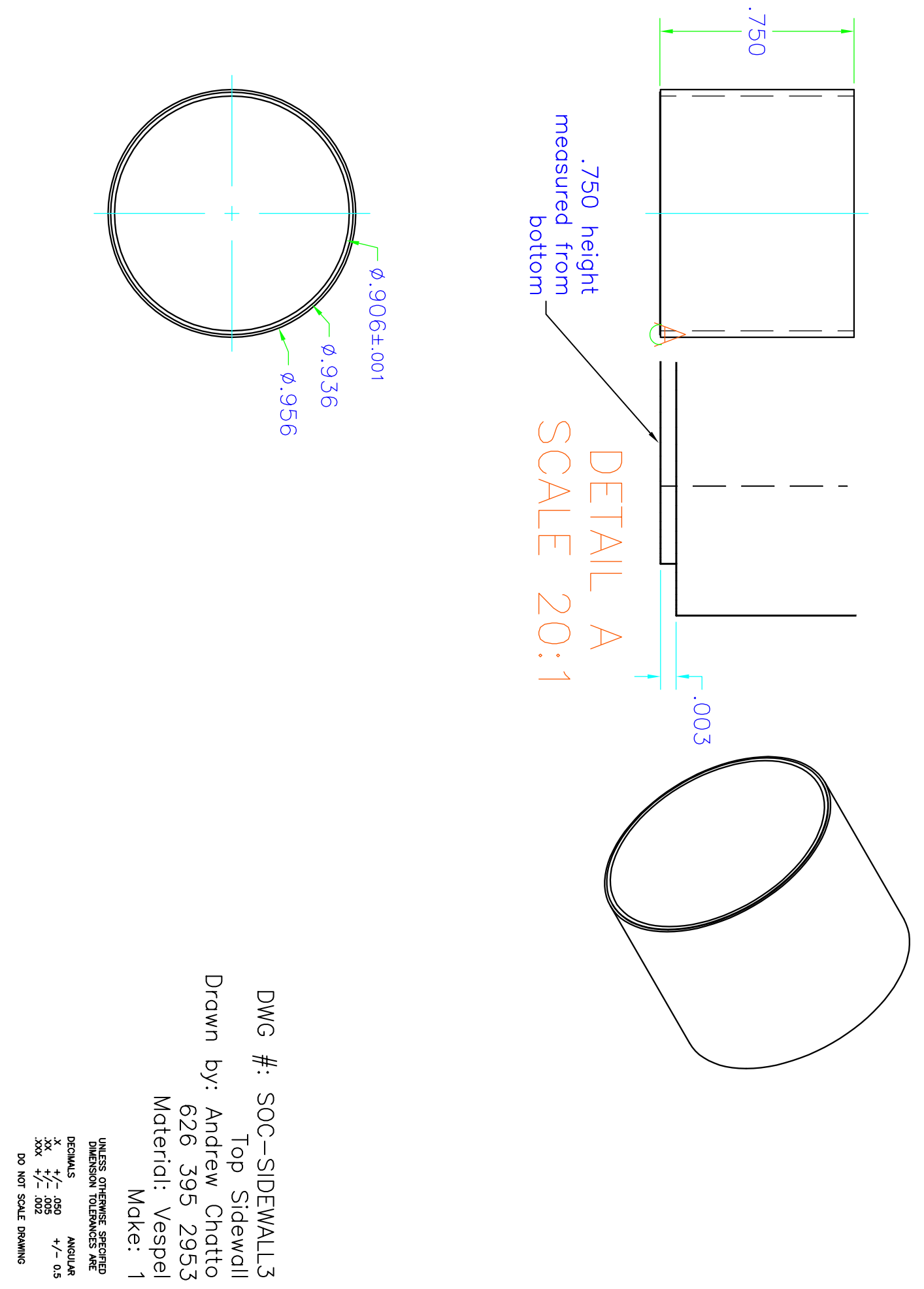

Figure A.7. The top piece of the Vespel sidewall. 

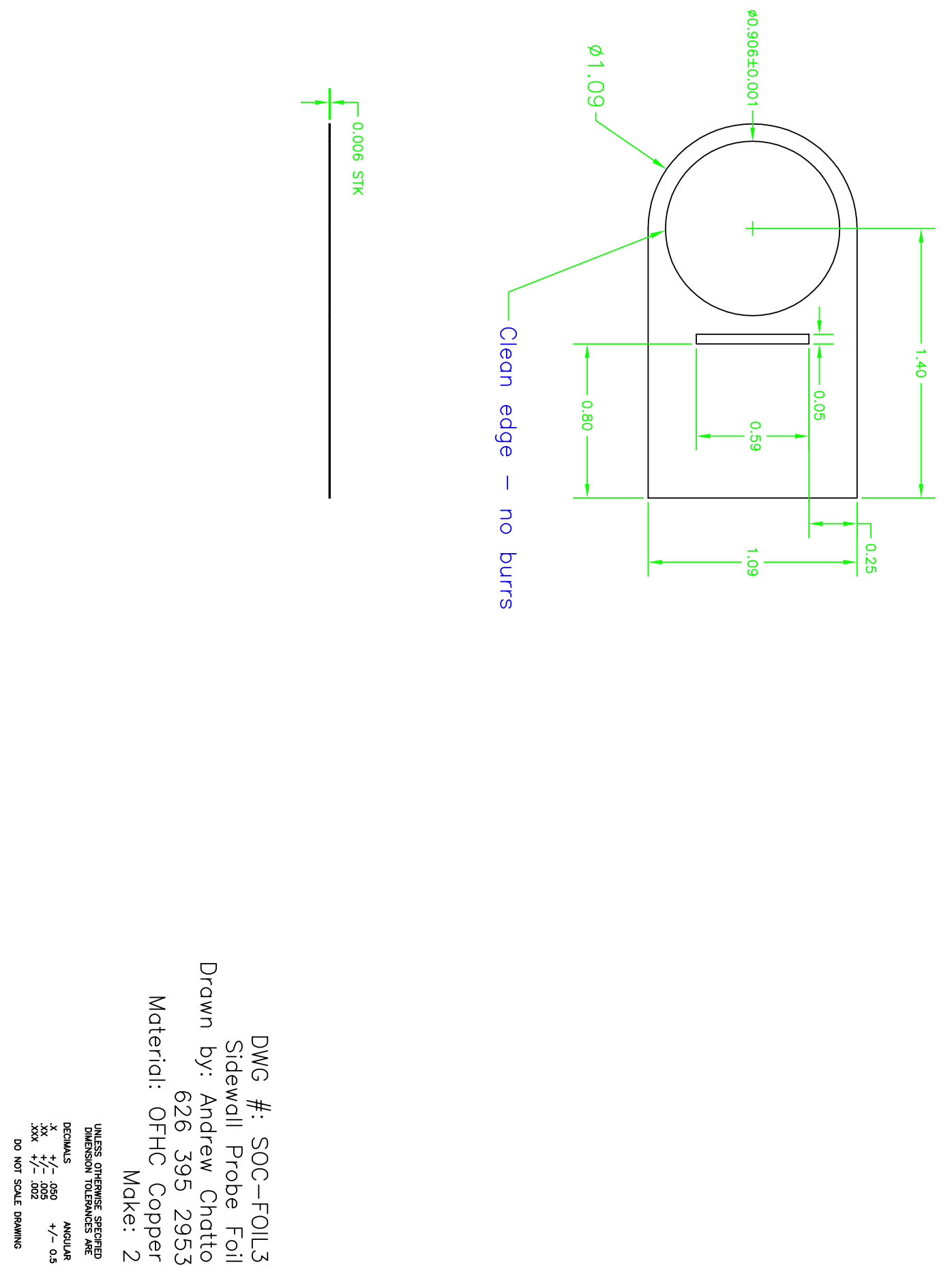

Figure A.8. The foil which was glued between sections of the Vespel sidewall. This is for the thicker foil; the thinner one was inherited from the DYNAMX project. 

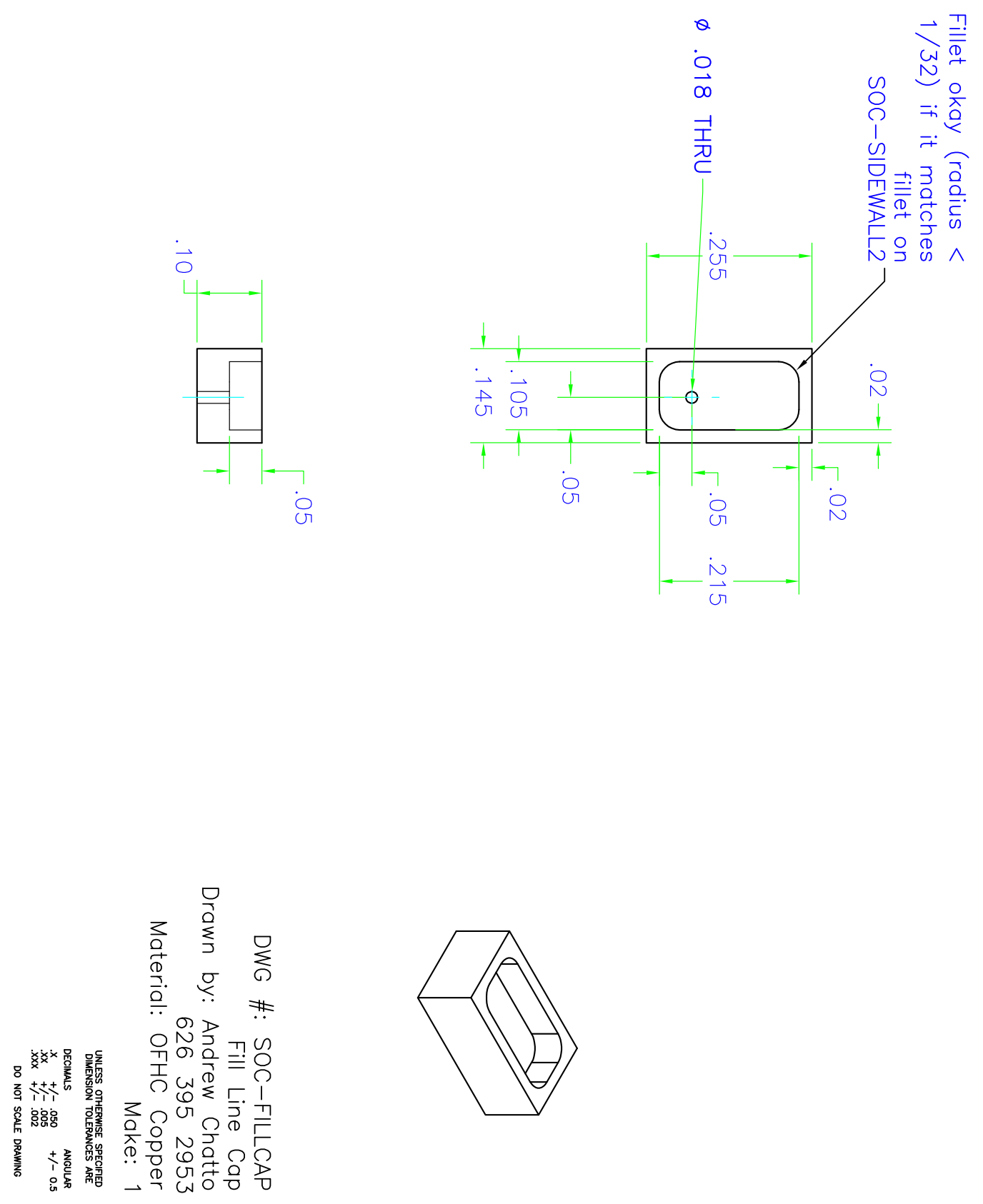

Figure A.9. The cap for attaching the fill line to the sidewall. 


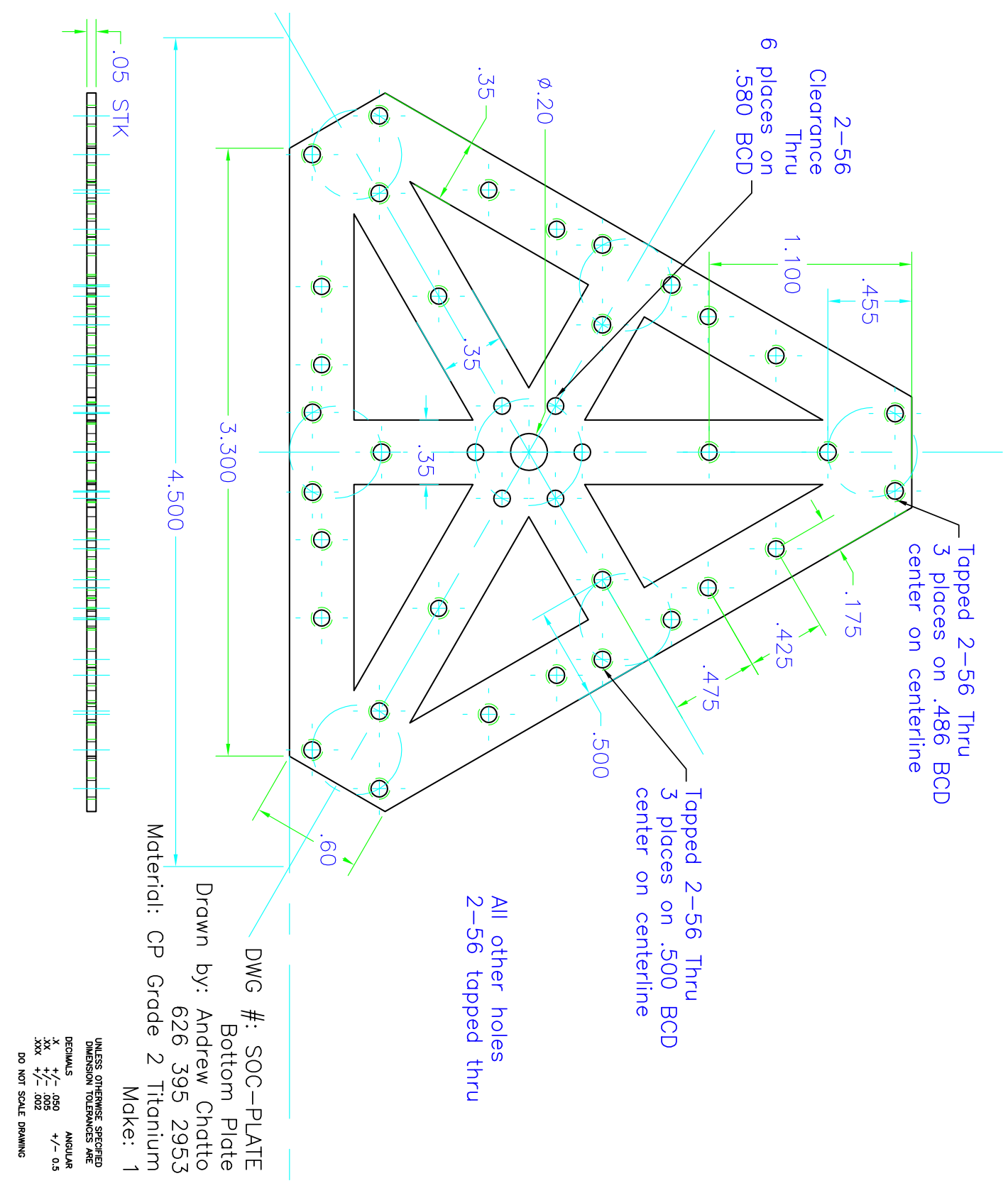

Figure A.10. The titanium mounting plate. 


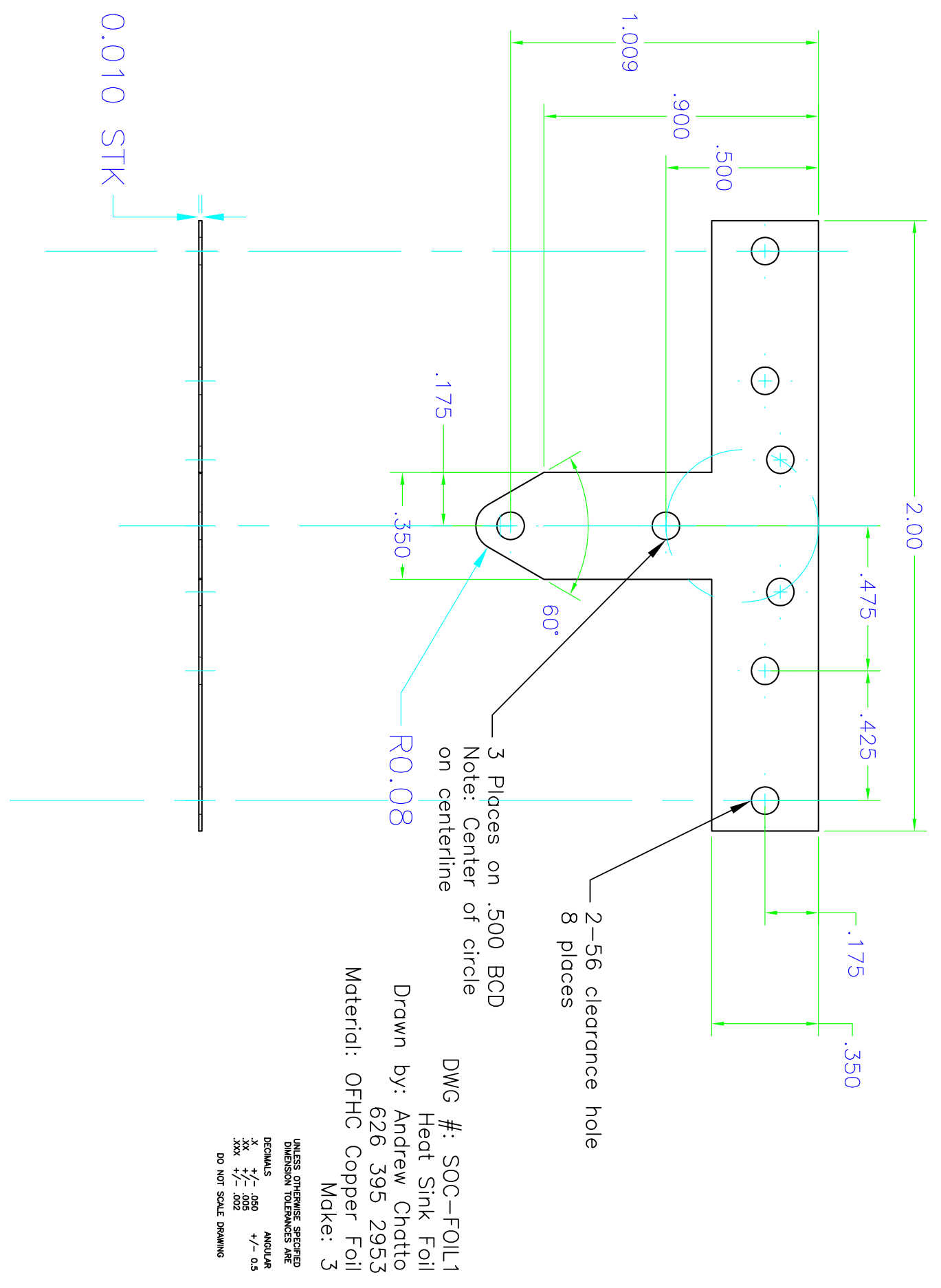

Figure A.11. The heat sinking foil. 


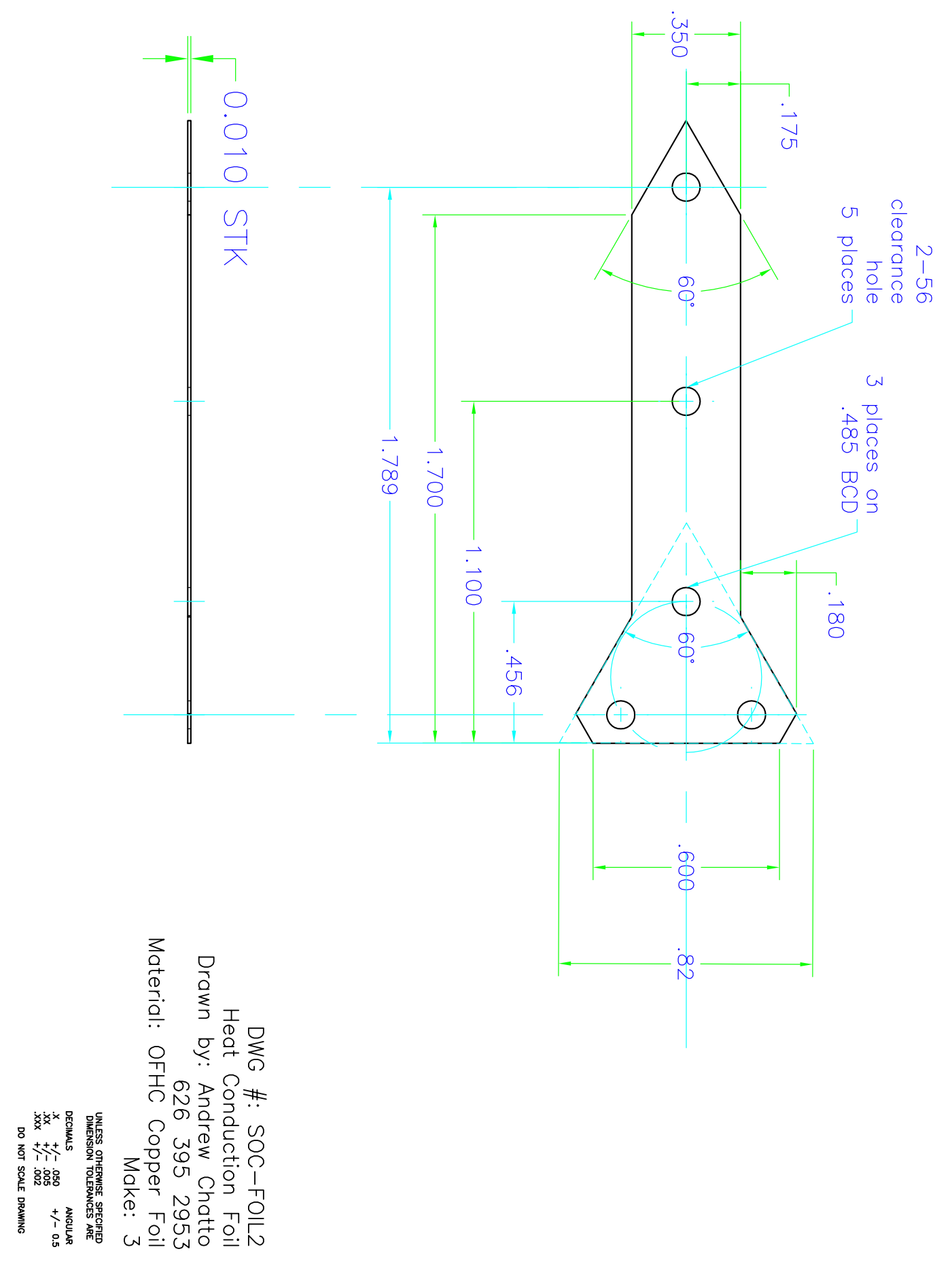

Figure A.12. The heat conduction foil. 

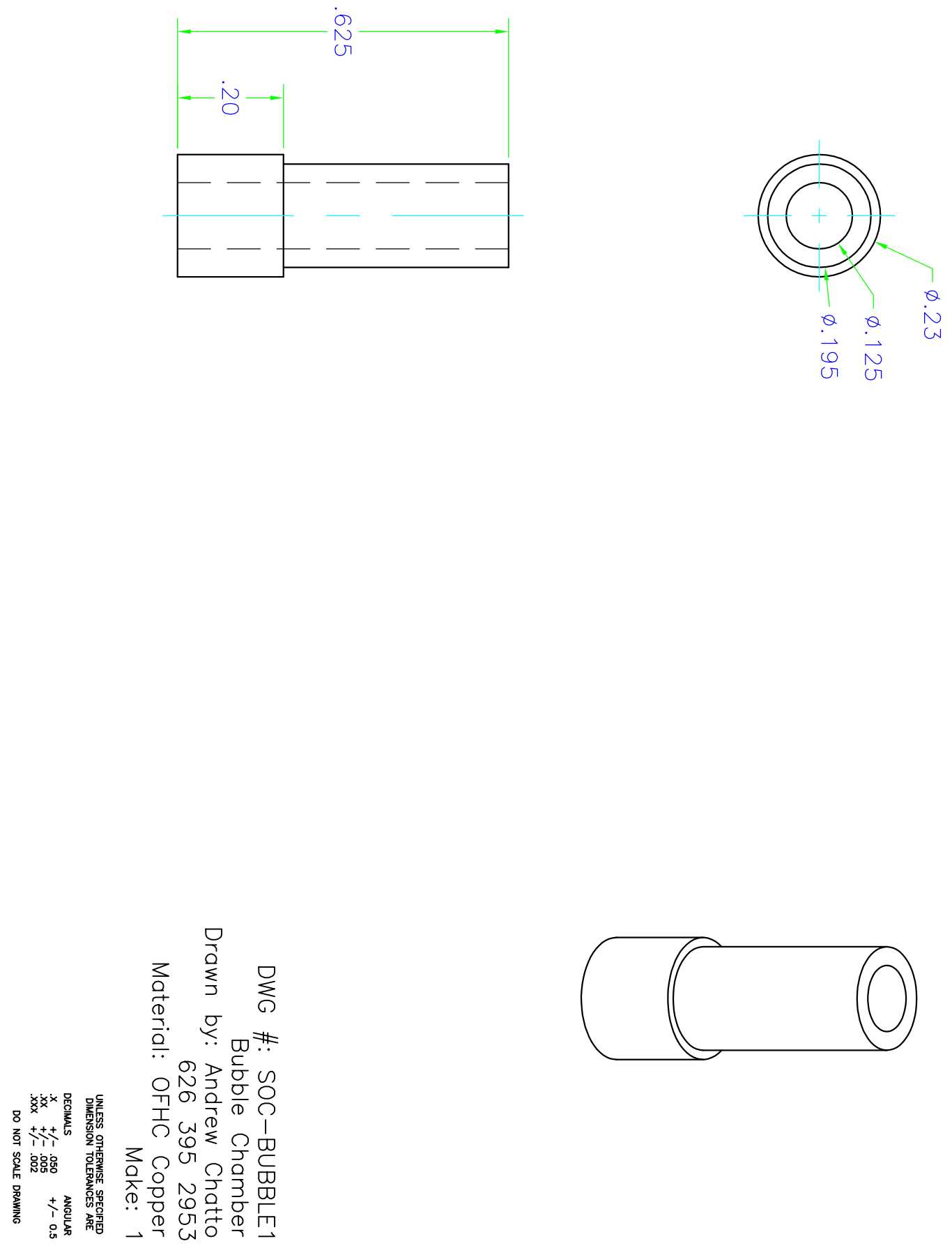

Figure A.13. The bubble chamber. 

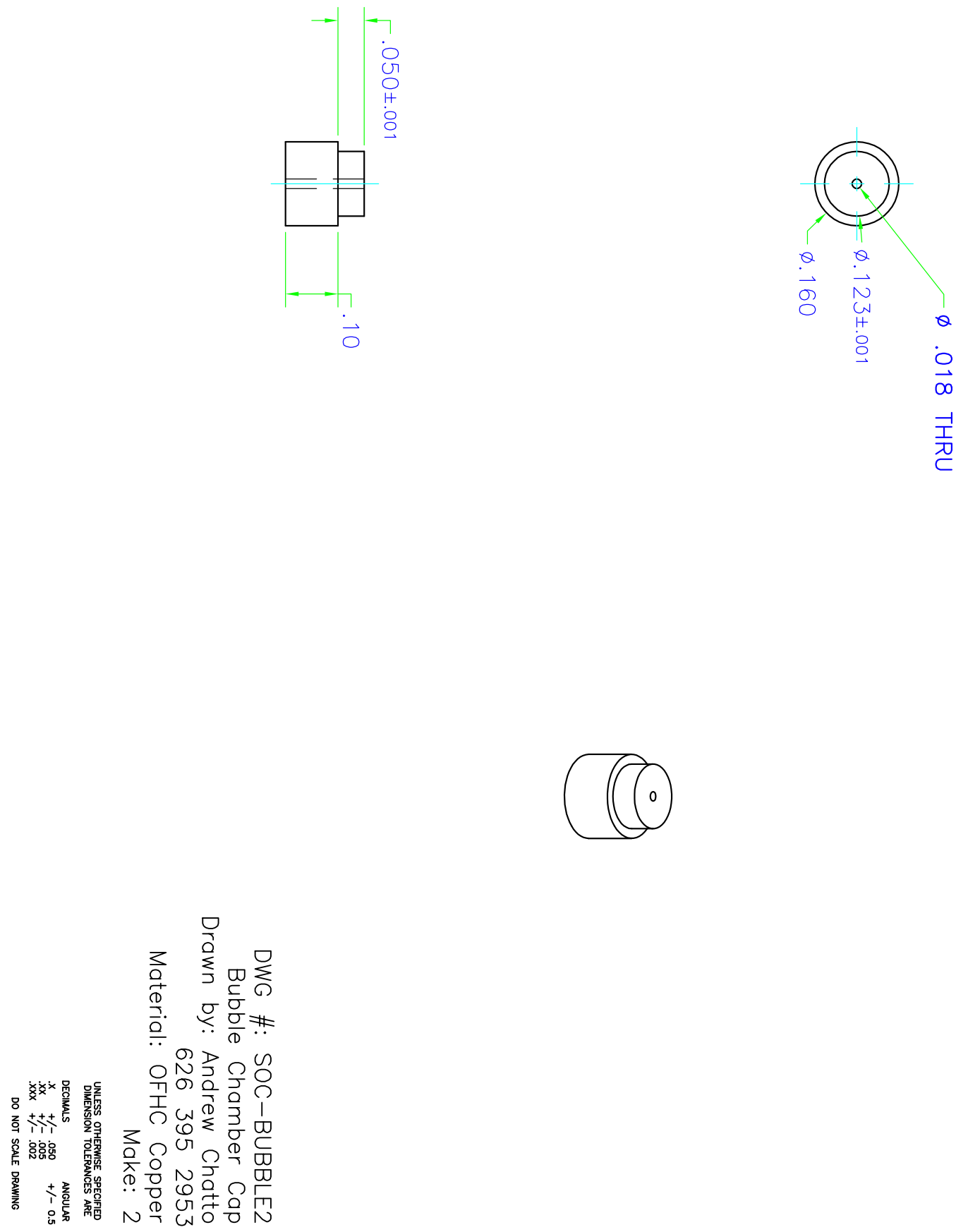

Figure A.14. The cap for the bubble chamber. 

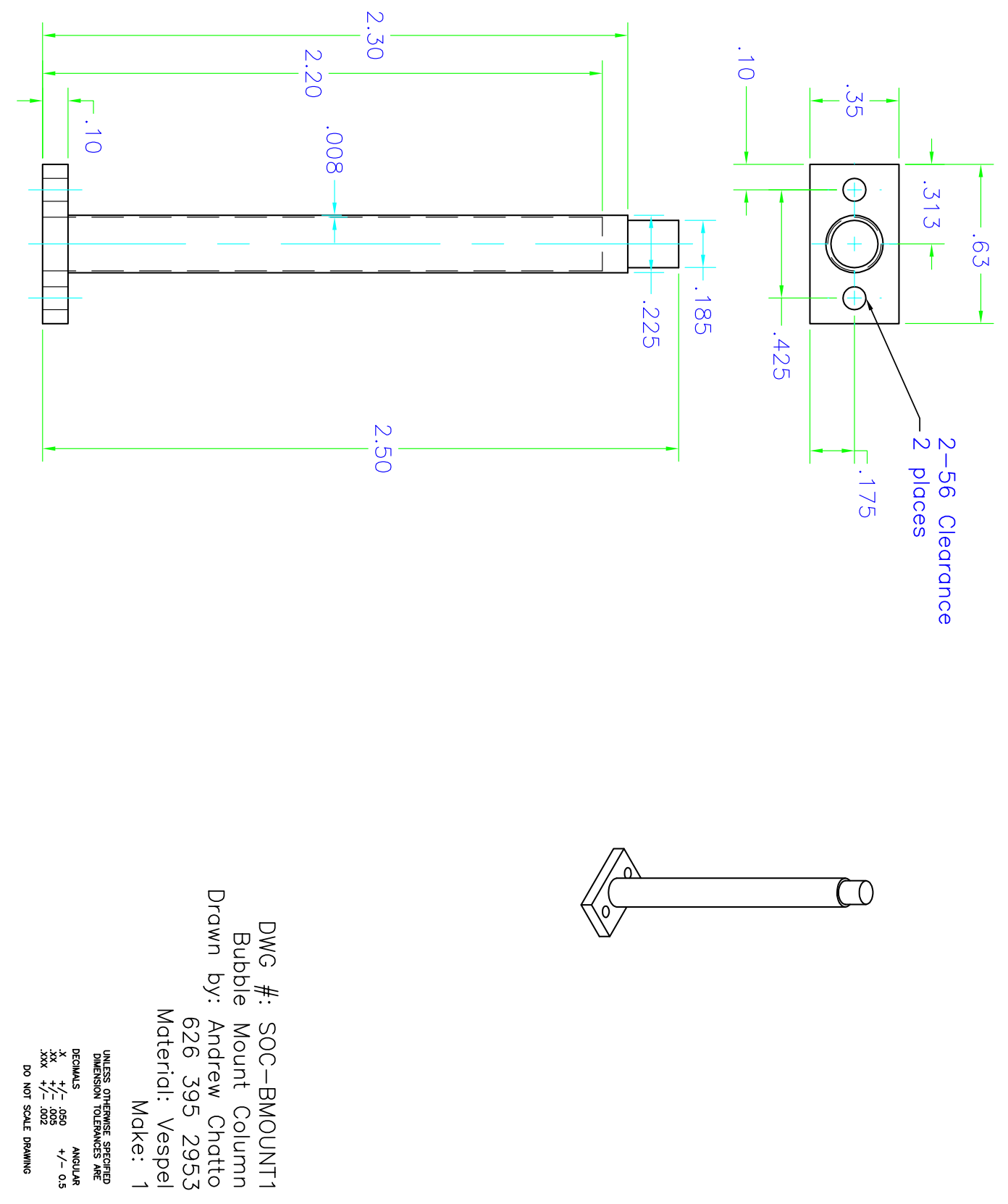

Figure A.15. Vespel thermal standoff for the bubble chamber and cryo-valve. 

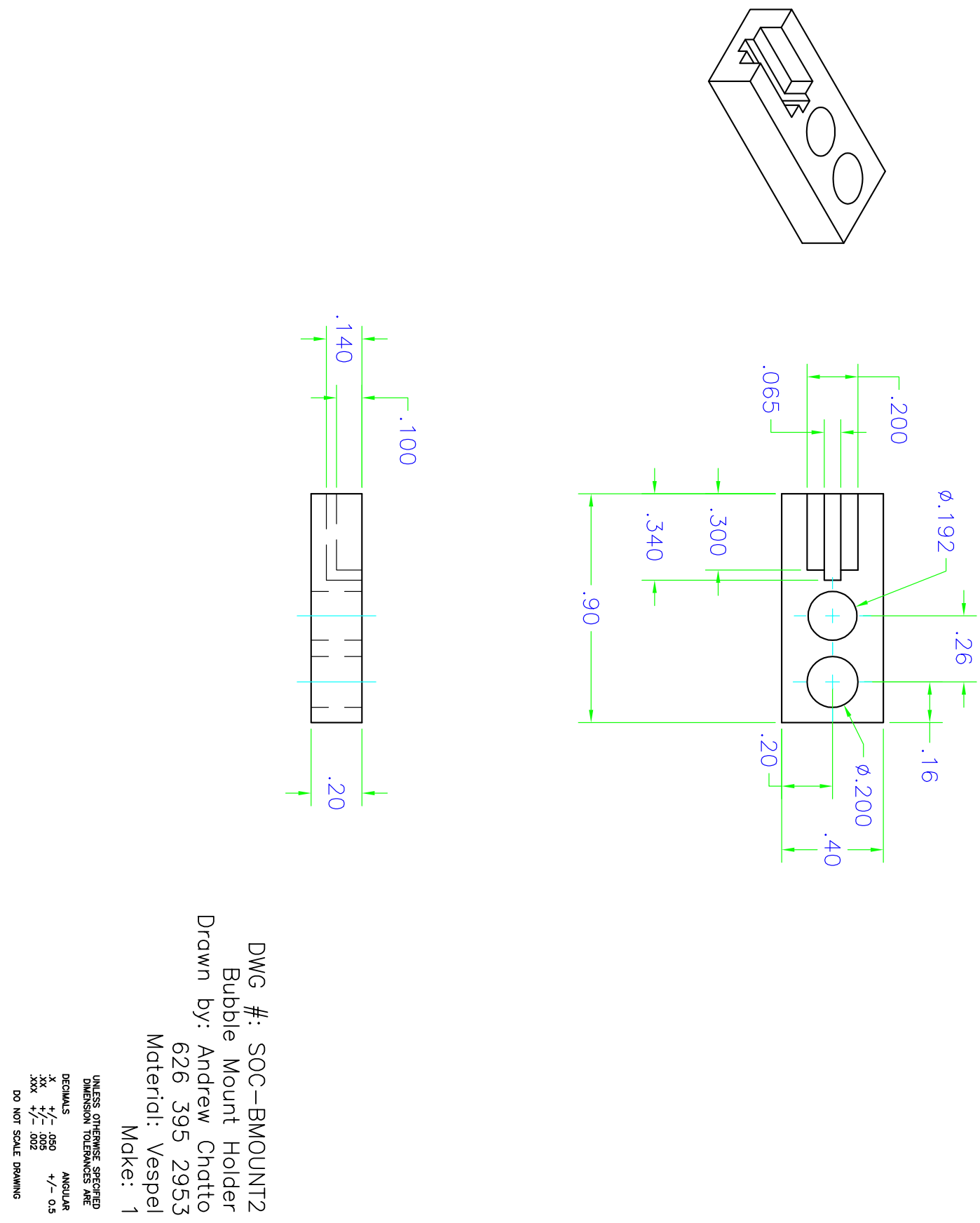

Figure A.16. Vespel mount for the bubble chamber and cryo-valve. 


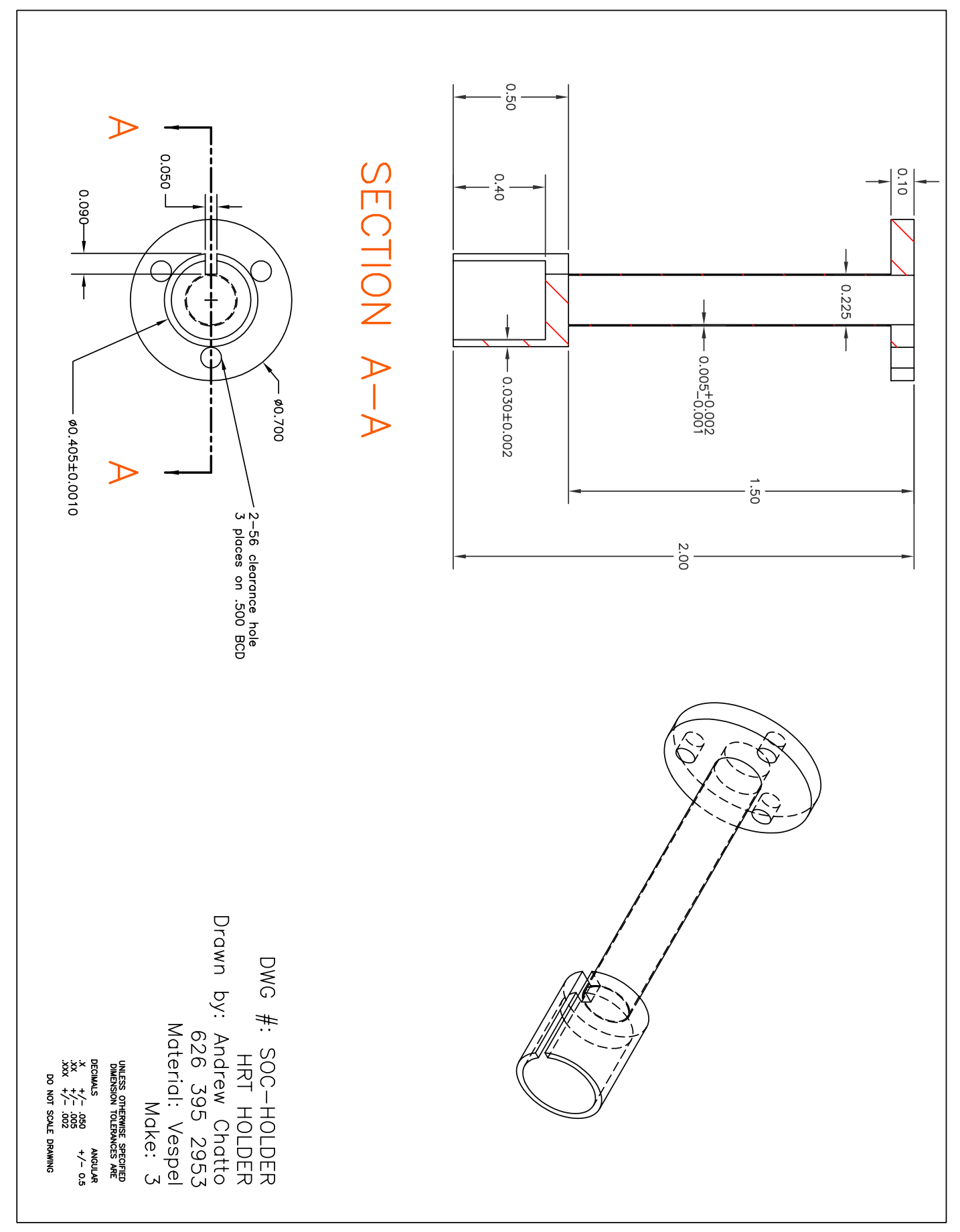

Figure A.17. Vespel mount and thermal standoff for an HRT for the bubble chamber. 